\title{
Handbook of Ostracod Taxonomy
}

by Henry V. Howe

LOUISIANA STATE UNIVERSITY STUDIES

Physical Science Series Number One 


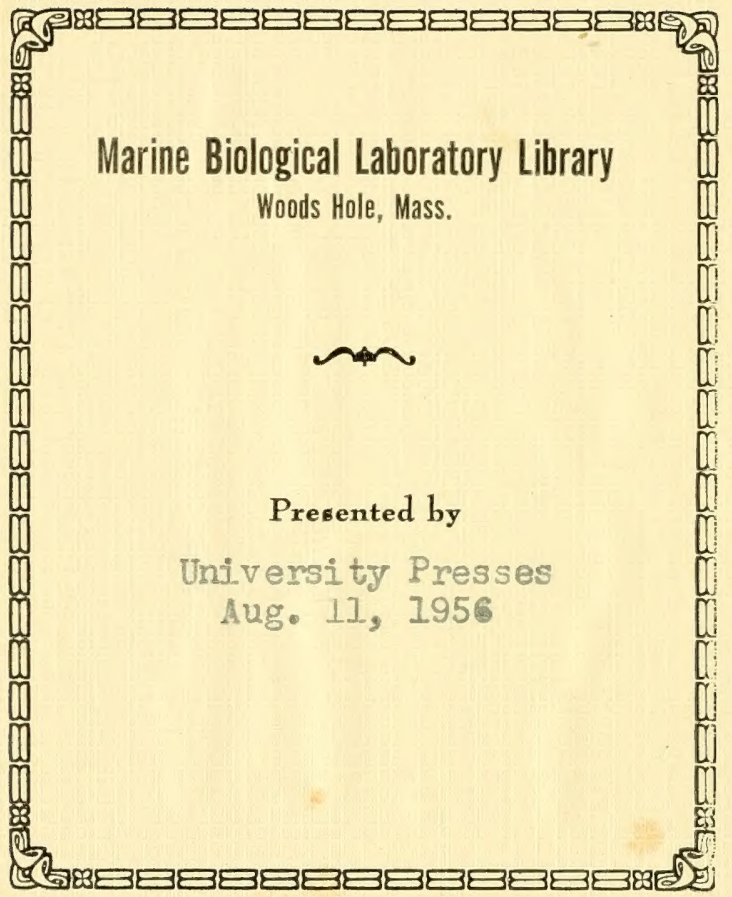


LOUISIANA STATE UNIVERSITY STUDIES

Physical Sclence Series

Vincent E. Parker, Editor

Number One

Handbook of Ostracod Taxonomy 


\title{
LOUISIANA STATE UNIVERSITY STUDIES
}

\author{
Richard J. Russell, General Editor
}

The Loulsiana State University Studies was established to publish the results of research by faculty members, staff, and graduate students of the University. Manuscripts of exceptional merit from sources other than aforementioned are considered for publication provided they deal with subjects of particular interest to Louisiana.

The Studies originally appeared as a unified series consisting of forty-two numbers published between the years 1931 and 1941. In 1951 the Studies was reactivated and is now being issued in the following series: Social Sciences, Humanities, Biological Sclences, and Physical Sclences. Other series may be established as the need arises.

The studies in each series w1ll be numbered only serially, without volume designation.

Requests for exchanges should be addressed to the Gift and Exchange Division, Louisiana State University Library, Baton Rouge. All other communications should be addressed to the Louisiana State University Press, Baton Rouge. 


\section{Handbook}

of

\section{Ostracod Taxonomy}

by

Henry V. Howe

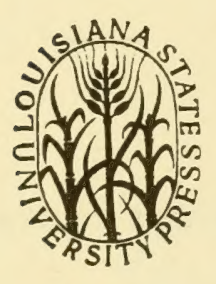

LOUISIANA STATE UNIVERSITY PRESS Baton Rouge 
Copyright 1955

By Loulsiana State University Press

Manufactured in the U.S.A.

Price: $\$ 5.00$ 


\section{DEDICATION}

TO

Mrs. Anne Jane Dyson, Senior Reference Librarian

Miss Marjorie E. Karlson, Senior Reference Librarian

Mr. George J. Guidry, Microfilm Librarian

Without whose enthusiastic assistance this volume could not have been completed.

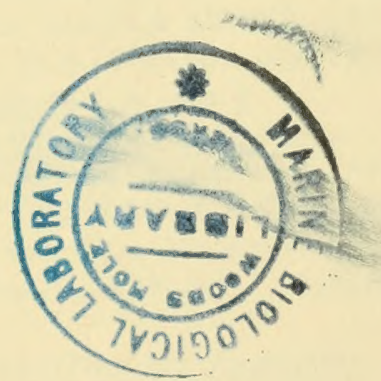



The following errors in the text have come to my attention largely through the kind assistance of I. G. Sohn and Stuart A. Levinson.

P. 3-3 lines up, insert "p. 194" after 1947.

P. 9-5 lines up, after p. 337, add: nom, nud?

P. 12-7 lines down, read A. semiplicata for A.simplicata.

P. 124-5 lines down, add MUTILA Neviani $1 \overline{928, \text { p. } 9=\text { Mutilus }}$ Neviani.

P. 140-8 lines up, read PARGRAPHYLUS for PARAGRAPHYLUS.

P. 159-11 lines up, add after subsurface, "brackish water."

P. 163 -5 lines up, read Aurigerites for Augurites.

P. 166-3 lines down, read Entomis for Estomis.

P. 175-5 lines down, read SINUSUE LLA for SINUSELLA.

P. 176-3 lines down, read SPINOVINA for SPINOVIA.

P. 180-6 lines up, insert SYNAPHE (S. mesozoica) Chapman 1914, p. 238, Triassic, Australia. Vide Sohn's letter of May 13. [Not seen].

P. 188-2 lines down, insert TRIGINGLYMUS [T.hyperochus] Blake 1950, p. 181 [Cytheridae-Cytherinae by Blake] Eocene, Alabama.

P. 188-12 lines up, read TUBULIBAIRDIA for TUBLIBAIRDIA.

P. 206-8 lines down, insert Blake, D. B. 1933. GOSPORT EOCENE OSTRACODA FROM LITTLE STAVE CREEK, ALABAMA. Journ. Paleo., vol. 24, p. 174-184, pls, 29-30. [Eleven n. sp., and Triginglymus, n. gen.]

P. 208-8 lines up, read Ardmorea for Armorea.

P. 222-5 lines down, add Chapman, Frederick 1914. AUSTRALIAN FOSSILS. A STUDENT'S MANUAL OF PALEONTOLOGY. George Robertson and Co., Melbourne, Sydney, Adelaide, Brisbane and London, p. 233-240. [reference from I. Sohn, not seen.]

P. 311-2 lines up, read Aurigerites for Augurites.

P. 324-6 lines up, read Sinusuella for Sinusella.

P. 331-4 lines down, read Tubulibairdia for Tublibairdia.

P. 341-9 lines up, read "2 of Kirkbya."

P. 382-2 lines down, add [preoccupied by Hermania Monterosato 1884, vide Neave 1939, p. 624].

P. 384-5 lines up read Semipetasus for Semiplastus.

P. 385 read SEMIPET ASUS for SEMIPLASTUS. 

When I first began an investigation of the Tertiary stracods of the Gulf Coast Region more than a quarter of a :entury ago, I quickly realized that the greatest problem acing an ostracod student was where to find the literature dealing with them. Our Library contained only a portion of the papers which I wished to see. Purchase of many books and articles was impossible because of their rarity. Microfilming had not become a standard practice, but I purchased ze of the first Leica cameras with a copying attachment, and began a practice of borrowing volumes and filming them myself. Then the U. S. Department of Agriculture Library set up a microfilm service, which opened the doors to the volumes contained in Washington. As more and more libraries have added microfilm departments, the task of assembling ostracod Iiterature has become much easier. At the present time, with a personal expenditure of some $\$ 4,500.00$, I have accumulated a microfilm library of well over 3,000 titles. How many books, papers and articles dealing with ostracods have been published, I do not know. My present estimate would be in the neighborhood of 5,000 titles. This estimate is based on the fact that I have partial references in my files to nearly 1,000 articles, which I have so far been unable to locate, because of incomplete bibliographic data, and I am sure that there are many of

\section{5}


which I have not as yet found a trace. Certainly there are many published in eastern Europe, and the USSR on which I have no information.

The continued accumulation of ostracod literature has made it abundantly clear that no library contains all of the literature on ostracoda. I have, therefore, indicated with each reference in my bibliography, the Library from which I obtained my copy.

One of the greatest problems in gathei'ing a library on ostracods is the unfortunate habit of many ostracod workers of so abbreviating their bibliographic references as to make them practically unfindable. They also are listed in a manner quite different from that used by librarians in cataloging. I have therefore appended to this volume, a list of the serials containing works on ostracod taxonomy, giving the titles as they are listed in the Union List of Serials. Or if they are not reported by the Union List, I have used the headings given by the Royal Society Catalogue of Scientific Papers, The Geological Record, London; Geological Literature on North America; or Bibliography and Index of Geology Exclusive of North America, as Iibraries normally have their books catalogued in accordance with these listings, which frequently differ greatly from the title page of a particular volume. Many journals have changed their titles several times, but will be catalogued usually only under the last title, or perhaps under the city in which published. With each listing, 
I have attempted to give the authors of articles on taxonomy, with the dates of their publication, which supplies something of a guide to ostracod workers on the importance of the various journals.

During the 19th Century it was customary to indicate the date on which a paper was presented before a learned society, and many references give this date instead of the actual date of publication. Again, during war years, many European journals fell behind in publication, and issued Journals for particular years at dates much later than normal. Sometimes these later dates are indicated on the title page, sometimes they are omitted, sometimes they are shown as footnotes to particular articles. Occasionally preprints of certain articles were issued several years before the full volume was issued. From this outline it is understandable why so many dates given in bibliographies are erroneous, occasionally by as much as ten years. These erroneous listings have caused me to purchase many microfilms which did not deal with ostracoda at all. I have attempted by every means at my command to verify the dates given in my Index and Bibliography, but feel that some error is unavoidable. I will appreciate readers calling my attention to any such errors.

Probably the greatest difficulty in obtaining microfilm copies of articles, is the failure of authors to give the record of pages and plates when citing an article. I have 
frequently had to purchase whole volumes on microfilm in order to find the description of a single species. I naturally found it necessary to have the title page of the volume in which an article was published photographed, but it has not always been easy to get libraries to do this, or to photograph the plates at the end of a volume, particularIy when no plates were indicated in the original reference. This difficulty, however, usually appears only with microfilm departments which have been recently established. While some libraries are not too well equipped for microfilming as yet, I wish to particularly express my appreciation to the following libraries for the prompt, courteous, exact service which they have rendered me over the years I have been gathering ostracod literature: the Universities of Illinois, California, Michigan, Minnesota, Wisconsin, Pennsylvania, Virginia, Washington, Chicago, Notre Dame, Ohio State University, Iowa State College, Yale, Harvard, Columbia, Cornell, the Library of Congress, and the New York State Department of Agriculture Iibrary.

I am also indebted to the following libraries and individuals for the loan of books or articles which were microfilmed by Mr. Guidry of the Louisiana State University Library for me: Harvard Museum of Comparative Zoology, Boston Public Library, Brown University, New York Public Library, Mt. Holyoke, Philadelphia Academy of Sciences, Stanford, Rice, 
Texas, Princeton, The John Crear Library, The United States Geological Survey, Stuart Levinson, Morton Stephenson, Harbans Puri, and Tetsuro Hanai.

I gratefully acknowledge much help from the following, who have over the years exchanged publications with me, sent me holotypes or topotypes of their species, or have made special collections from topotype localities for me, or who have located publications for me that I needed in this work: Charles Ivan Alexander, Dallas, Texas; R. S. Bassler, U. S. National Museum, Washington, D.C.; the late Daniel Blake, Baton Rouge, Louisiana; the late Dr. J. H. Bonnema, Groningen, Netherlands; Bedrich Boucek, Prague, Czechoslovakia; the late Frederick Chapman of Australia; Robey H. Clark, New Orleans, Louisiana; Alan Cheetham, Baton Rouge, Louisiana; H. N. Coryell, Columbia University, New York; Robert Crouch, Houston, Texas; Carey Croneis, Houston, Texas; Christian F. Dohm, New York City; Richard Edwards, Gainesville, Florida; A. D. Ellis, Jr., Houston, Texas; Harold N. Fisk, Houston, Texas; Julius Garrett, Houston, Texas; N. Grekoff, Rueil-Malmaison, Seine-et-Oise, France; Wade Hadley, Jr., Caracas, Venezuela; Tetsuro Hanai, Tokyo, Japan; Reginald W. Harris, Norman, Oklahoma; Gunnar Henningsmoen, Oslo, Norway; Ivar Hessland, Uppsala, Sweden; Bernard Hill, New Orleans, Louisiana; Keith Hussey, Ames, Iowa; Merle C. Israelsky, Pasadena, California; N. de B. Hornibrook, Wellington, New 
Zealand; Leo Hough, Baton Rouge, Louisiana; Marshall Kay, Columbia University, New York; Mrs. Betty Kellett Nadeau, Boronia, Victoria, Australia; Robert V. Kesling, Ann Arbor, Michigan; A. J. Key, Utrecht, Netherlands; John M. Law, New Orleans, Louisiana; L. W. LeRoy, Golden, Colorado; Stuart A. Levinson, Houston, Texas; Frank E. Lozo, Houston, Texas; James D. Mclean, Jr., Alexandria, Virginia; Kenneth - Mclaughlin, Missoula, Montana, Raymond C. Moore, Lawrence, Kansas, Grover Murray, Baton Rouge, Louisiana; Raymond E. Peck, Columbia, Missouri; Vladimir Pokorny, Prague, Czechoslovakia; Harbans S. Puri, Tallahassee, Florida; Lloyd M. Pyeatt, Houston, Texas; W. T. Rothwe1l, Long Beach, Califurnia; Giuliano Ruggieri, Bologna, Italy; Ruth A. M. Schmidt, Washington, D. C.; Harold W. Scott, Urbana, Illinois; James V. Sexton, San Antonio, Texas; I. G. Sohn, Washington, D. C.; Nils Snjeldnaes, Oslo, Norway; Morton B. Stephenson, Houston, Texas; Frederick M. Swain, Minneapolis, Minnesota; Frank M. Swartz, State College, Pennsylvania; P. C. Sylvester-Bradley, Sheffield, England; Clemencia Tellez-Giron, Mexicn City; Will is L. Tressler, Washington, D. C.; Erich Triobel, Frankfurt a-M, Germany; W. V. Van den Bold, Point Fortin, Trinidad; J. Elizaboth Van Veen, Groningrr, Netherlands; Robert O. Vernon, Tallahassen, Florida; Leo M. Weingeist, Caracas, Venezuela; the late Louis Wilbert, Jr., Baton Rouge, Louisiana; and Raymond D. Woods, Houston, Texas. I also wish gratefully to 
acknowledge the assistance of Miss Helen Forsburg, Senior Librarian of the School of Geology, who has taken particular pains to keep me posted on new acquisitions to our library that dealt with ostracoda.

In the preparation of this Handbook I have gratefully made use of the 6 bibliographies of Paleozoic crustacea compiled by Vogdes 1889, 1890, 1893, 1895, 1917, 1925; the monumental compilation of recent ostracoda by G. W. Muller 1912, in Das Tierreich; Bassler and Kellett's 1934 Bibliographic Index of Paleozoic Ostracoda; Branson's 1948 Bibliographic Index of Permian Invertebrates; the Bibliography of Paleontology and Stratigraphy compiled by Anna Samarodova in PROBLEMS OF PALEONTOLOGY, vols.I-V, Moscow 1935-1939, Agnew's 1942, 1944 Bibliographic Index of new genera and families of Paleozolc Ostracoda since 1934; Levinson's 1953 Bibliography and Index to New Genera of Ostracoda 1950-1952; the Zoological Record, the Zentralblatt fur Mineralogie, Geologie, und Paleontologie, as well as the bibliographies accompanying each of the articles in my personal library. Moreover I have checked the generic and subgeneric names against the list given by Neave's 1939-1950 NOMENCLATOR ZOOLOGICUS for verification of the homonyms listed. There are probably more homonyms to be discovered, as a surprisingIy large number of ostracod generic and subgeneric names are not listed in Neave. 
In addition to the papers containing subgeneric, generic and other taxonomic terms, I have included in the bibliography, the papers in which the genotype of subgenotype was described, in case such type was by another author. No attempt is made to list species other than those used as genotypes or subgenotypes, as such a catalogue would be of vast proportions. My partial card index, based on about 2,000 of the more than 3,000 papers in my library which I have cross-indexed by species contains about 19,000 cards. I would estimate that more than 30,000 specific names have been applied to the ostracoda, and confusion in places is very great. This is occasioned by the fact that for the most part zoologists, working with Recent ostracoda have paid practically no attention to the work done by paleontologists. The paleontologists working with Paleozoic ostracoda have until most recently used an artificial classification, not based on the living organisms, and in many cases have oriented the carapaces in reverse, with the posterior shown as anterior, the left valve as right, and in some cases have even illustrated them upside down. As a result my card index contains upwards of 500 homonyms which may be attributed directly to the fact that workers in one field were not familiar with the work done in the other. In this Handbook, I have attempted to include not only all valid ostracod terms which have been used, but those 
terms originally ascribed to ostracods, but later transferred to Conchostraca or other groups. It is interesting to note that in the 75 years following 0 . F. Muller's description of Cypris, in 1776, only about a dozen taxonomic terms were developed for the ostracoda. The past hundred years have seen the number of terms grow to approximately 1200. Agnew 1942, p.756 noted that Bassler and Kellett 1934 listed less than 200 paleozoic genera, and listed 167 additional genera or subgenera from the Paleozoic for the next 8 years. Levinson $1953, \mathrm{p} .51$ noted the addition of 71 new genera and subgenera during the years 1950-1952, remarking that it was a large increase from the 31 new genera and subgenera in the years 1947-1949. The rate is still increasing. Unfortunately many of the new genera turn out to he homonyms or synonyms. It is hoped that this Handbook will make available to authors of new genera much needed information which will tend to reduce the number of unnecessary names. It should be pointed out that the age given for each fossil genus is in most cases the age assigned by the original author. I have attempted to give the age assigned to the type species, in case the type species was described by an earlier author. In a number of cases the author did not give the age, but merely the formation or locality from which it was described. In such cases for American ostracod genera, I have attempted to follow Wilmarth's Lexicon of geologic names of the United States, U. S. Seol. Survey Bulletin 896. 
for the age assignment. It is obviously impossible in many cases to determine whether some species listed actually came from the Miocene or from the Oligocene, or from Upper Ordovician, or Lower Silurian. No author could be sufficiently familiar with the precise age of all type localities, many of which are still in question. Barrande's "Silurian" ostracoda of Bohemia came from various portions of the Paleozoic. Some of these have been settled by later workers such as Pribyl, and in such cases I have taken the later assignment. In other cases, I have followed Barrande's assignment to the portion of the "Silurian" to which he assigned the species. Dr. F. M. Swain under date of June 8, 1954 has kindly written me:

"Ulrich's 'Lower Silurian' ostracodes are nearly all, if not all, post-Chazyan Ordovician (cf. Schmidtel1a)."

For these genera I have therefore followed Swain, rather than Ulrich in the age cited.

This volume is not an attempt to give a complete bibliography of ostracoda. Perhaps in future years, if current ostracod workers will kindly call my attention to papers which I might otherwise overlook, and if I can persuade Dr. Stuart Levinson to join me in such an undertaking, thus making available the large ostracod library which he and the Humble $0 i 1$ and Refining Company have amassed, we together might be able to come fairly close to completeness. 
It is likewise not a compendium of terms used in description. Students needing to become familiar with the anatomical terminology of ostracods would do well to turn to G. W. Müller's great 1894 monograph, Sars' 1922-1928 Account of the Crustacea of Norway, vol.9, or Hoff's 1942 Ostracods of Illinois. For the terminology of the carapace, students will find illustrations and definitions in G. W. Müller 1894; Sylvester-Bradley 1941, 1948d; Bold 1946; Hornibrook 1952; Zalanyi 1929; or Ruggieri 1950a. Kesling's 1951 Terminology of ostracod carapaces should be sufficient for the Palconzoic student. All students of ostracoda, Recent or fossil should be familiar with Triebel's 1941 discussion of their morphology and ecology.

I have instead attempted to give as completely as possible all references which I have noted anywhere in the literature on ostracod taxonomy regardless of whether I possessed the publication or not. I believe that my library is complete enough to have at least some mention of nearly all of the ostracod subgenera, genera, subfamilies, families and higher taxonomic units. As a further check of my completeness in this respect, I have sent copies of the Index portion to Raymond C. Noore, editor in chief of the Treatise on Paleontology, H. W. Scott, Chairman of the Paleozoic committee on Ostracods for the Treatise, Frederick M. Swain, and Stewart Levinson, both distinguished ostracod experts and members of the Treatise committee on ostracods. From 
them, and particularly from Levinson, I have received valuable comments, notations of omissions and help with the missing literature. As a consequence of their laborious and unselfish assistance, I feel that I am able to present this Handbook of Ostracod Taxonomy to future workers, with the assurance that it is the best which may be done in American Libraries at the present time. Less than 10 essential papers, so far as I have been able to learn, are missing from my personal library, and all are so indicated in both the Index portion and in the Bibliography. 
INDEX TO TAXONOMIC TERMS 

ABDITOLOCULINA [A, insolita] Kesling 1952,p.765 [Hollindae by Kesling] Devonian, Ohio.

ACANTHOBUS G. W. Muller 1900,p.104; 1912,p.428 (misspelling of Acanthopus Vernet).

ACANTHOCYPRIS [A. bicuspis] Claus 1893,p.196-201 [Cypridae by Claus] Recent, South America. [ = Strandesia Stuhlmann 1888, by G. W. Müller 1912,p.187].

ACANTHODELLA [A, terciocornuta] Zaspelova 1952,p.201 [Drepanellidae-Nodellinae by Zaspelova] Devonian, USSR.

ACANTHOPUS [A. resistans and A. elongatus] Vernet 1878, p.506-526 [non Klug 1807] [A. resistans $=$ Cytherissa lacustris by Sars 1925,p.155; $\underline{\text { A. }}$ elongatus $=$ Limnocythere sancti-patricii by $G$. W. Muller 1912,p.332.] Non Acanthopus Klug 1807 , Hym; Oken 1816, Pisces; Dahl 1823, col. etc., fide Neave 1939, vol.1,p.20.

ACANTHOSCAPHA [Beecherella navicula Ulrich 1891,p.203,pl.2, f.8,9] Ulrich and Bassler 1923,p.319. [Beecherellidae by UEB] Devonian, New York.

ACOCYPRIS [A. capillata] Vavra 1895,p.14,15 [Cypridae-Cyprinae by G. W. Muller 1912,p.196] Recent, Zanzibar. 
ACRATIA [A. typica] Delo 1930,p.174 [Bairdiidae by Delo] Pennsylvanian, Texas.

ACRONOTELIA [A. shideleri] Ulrich and Bassler 1923,p.299, 302 [Primitiddae by Bassler and Kellett 1934,p.19, 146; Hollinidae-Ctenentominae by Schmidt 1941, p.34; Acronotellidae by Henningsmoen 1953,p.234, 269] Silurian, Indiana.

ACRONOTELLIDAE Swartz 1936,p.554 a family to include Acronotella, Eoconchoecia, Monoceratina, Mooreina.

ACROSSULA [A. u-scripta] Kummerow 1953,p.42 [Piretellidae by Kummerow] Devonian, Germany •

ACROTONELLA Peneau 1927,p.112 [?= Acronotella by Bassler and Kellett $1934, \mathrm{p} \cdot 146]$

ACTINOCYTHEREIS [Cythere exanthemata Ulrich and Bassler 1904, p.117,pl.36,f.1-5] Puri 1953a,p.178 [Cytheridae] Miocene, Maryland. Range Eocene to Recent.

ACUTICYTHEREIS [A. Laevissima] Edwards 1944,p.519

[Cytheridae by Edwards] Miocene, North Carolina. $[=$ Campylocythere Edwards, by Malkin 1953,p.764]

AECHMINA [A. cuspidata] Jones and Holl 1869,p.217 [Leperditidae by Ulrich 1894,p.632; Cytheridae by Jones 1901,p.147; Primitiidae-Primitinae by Bassler and Kellett 1934,p.19; Aechminidae by Swartz 1936, p.564; Drepanellidae-?Aechmininae by Henningsmoen 1953,p.234,269] Silurian, England. 
AECHMINARIA [A. nodosa] Coryell and Williamson 1936,p.5

[Primitiidae by CEW; Aechminidae by Agnew 1942, p.757; Drepanellidae-Aechmininae by Henningsmoen 1953,p.234,269] Silurian, Indiana.

AEChMINELlA [A. trispinosa] Harlton 1933,p.19 [Beyrichiidae by Harlton; Kirkbyidae by Swartz 1936,p.549;

Drepanellidae by Kellett 1936,p.771; Kloedenellidae-Kloedenellinae by Henningsmoen 1953,p.243,271]

Pennsylvanian, Oklahoma.

AECMMINIDAE Swartz 1936,p.553, a family for the genera Aechmina, Paracchmina, and on 0.584 for Iindsayella, Cornulina, Aechminaria.

AECHMININAE Boucek 1936,p.53, a subfamily of Primitiidae for the genera Aechmina, Paraechmina and Novakia.

AFROCYPRIS [A. barnardi] Sars 1924b,p.206 [Cypridae by Sars] Recent Ovamboland, South Africa.

AFROCYTHERE [A. rostrata] KIie 1935,p.63 [Cytheridae by Klie] Recent, French West Africa.

AGLAIA [A. pulchella] Brady 1868 Fonds vol.1,p.90 [non Aglaia Renier, 1804 (Mollusca); non Aglaia Swainson 1827 (Aves) = Paracypris by G. W. Müller, but is renamed Aglaiocypris by Sylvester-Bradley 1947] Recent, Coast of Sicily. (Brady's figures look very similar to those of Cushmanidea). 
AGLAIELLA [A. stagnalis] Daday 1910b,p.557 [near Aglaia and Paracypris by Daday] Brackish water, Egypt.

AGLAIOCYPRIS [Aglaia pulchella Brady] Sylvester-Bradley 1947,p.194, nom.nov. [Cypridae by S-B] Recent, Sicily •

AGNOSTUS [of Brongniart 1822, a Trilobite], used M'Coy 1846, p.67, Vanuxem 1842,p.80, etc., for species which were later placed in the Ostracoda.

ALANELIA [A. bohemica] Boucek 1936,p.71 [Alanellidae by Boucek; doubtful ostracod by Agnew 1942,p.756; ?Alanellidae by Henningsmoen 1953,p.247,273.] Silurian, Bohemia.

ALANELLIDAE Boucek 1936,p.71; Henningsmoen 1953,p.247,265, 273. Henningsmoen places here: Alanella, Vltavina, Berounella, Tricornina, ?Boucekites, ?Pribylites, and ?Pribylites (Parapribylites).

ALATACYTHERE [Cythereis(Pterygocythereis?) alexanderi Howe and Law $1936, \mathrm{p} \cdot 1+2, \mathrm{pl} \cdot 4, \mathrm{f} \cdot 23 ; \mathrm{pl} \cdot 5, \mathrm{f} \cdot 5$; (non Morrow 1934) = Alatacythere ivani Howe 1951a,p.538, nom.nov.] Murray and Hussey 1942,p.168 [Cytheridae by MEH] Oligocene, Mississippi.

ALBANELIA [A. gouldi] Harris and Lalicker 1932,p.397 [Beyrichiidae by HEL; = Amphissites by Bassler and 
Kellett 1934,p.149; but is recognized by Henningsmoen 1953,p.272 and assigned to Kirkbyidae]

Carboniferous, Kansas. [non Mueller 1914, col., fide Neave 1939,vol.1,p.103.]

ALEXANDRELIA [Bairdia napthotscholana Liventhal 1929]

Schweyer 1939,p.96 [ = Cytherissa by Bronstein 1939, p.336] Tertiary, USSR. [non Chevreaux 1911, crust.; Tonnoir 1926, dipt., fide Neave 1939, vol.1,p.110]

ALIOSTRACA [A. fimbriata"(Ulrich and Bassler)" in Bassler 1932,p.236,pl.27,fig.5 [Uncertain family by Henningsmoen 1953,p.273] Mississippian of Tennessee. (only description in explanation of plate).

ALUTA [A. flexilis] Matthew 1896,p.198 [an ostracod by Matthew; but referred to Conchostraca by Ulrich and Bassler 1931,p.49-51] Cambrian, New Brunswick.

ALVEUS [A. depressus] Hamilton 1942,p.717 [Cytherellidae by Hamilton] Permian, Texas. [Spelled Alvenus on plate 110 ,figure $6 \mathrm{a}-\mathrm{b}]$

ALVENUS Agnew 1944,p.218, following spelling given on Hamilton's plate, rather than spelling given in description of genus and type species. = Alveus Hamilton. 
AMONOCYTHERE Sohn 1951,p.64 [Mis-spelling of Anomocytheridea Stephenson]

AMPHICYPRIS [A.nobilis] Sars 1901,p.16-18 [Cypridae by Sars] Recent, grown from Argentine mud. [ = Eucypris Vávra 1891 by Mïller 1912,p.173]

ANPHISSITES [A. rugosus] Girty 1910,p.236 [Kirkbyidae by Bassler \& Kellett 1934,p.149 and Henningsmoen 1953, p.245,272] Mississippian, Arkansas. [Range from 135 reported species Devonian to Permian] Amphissites was redefined by Knight 1928,p.246-250, 258,259 .

ANPHISSITINAE Cooper 1941,p.47 (Subfam. of Kirkbyidae) for Amphissites, Ectodemites, and Polytylites. Hot recognized as needed by Henningsmoen 1953,p.245, 272.

AMPHIZONA [A. asceta] Kesling and Copeland 1954,p.154 [Kirkbyidae by KEC] Devonian, New.York.

AMPLOCYPRIS [A. sinuosa] Zalányi 1944,p.26,14] [Cypridae-Cyprinae by Zalányi] Neogene of Hungary.

AMPULOIDES [A. vermeosa] Polenova 1952,p.138 [Incertae sedis by Polenova] Devonian, USSR. 
ANCHISTROCHELES [A. fumata G. S. Brady M.S. =A.fumata Brady $1890, \mathrm{p} \cdot 497, \mathrm{pl} \cdot 3, f \cdot 13,14$, text figs.1-9 by original designation] Brady \& Norman 1889,p.110 [Cypridae by BEN.; Bairdiidae by Brady 1890, p. 498 and by G. W. Müller 1894,p.266-267; Nesideidae by G. W. Müller 1912,p.250] Recent, Fiji.

ANISOCHILINA [A. punctulifera] Teichert 1937,p.106

[Leperditidae by Teichert; Leperditiidae-Leperditiinae by Henningsmoen 1953,p.274] Ordovician, Greenland.

ANKUMIA [A. bosqueti] Veen 1932,p.359,360 [Cytherellidae by Veen] Cretaceous of South-Limburg.

ANOMOCYTHERIDEA [Cytheridea floridana Howe \& Hough, in Howe et.al.1935,p.10,pl.2,f.15,16,18; pl.4,f.6,10] Stephenson 1938,p.141. [Cytheridae by Stephenson] Miocene, Florida. [ =Cyprideis Jones 1857, by Pokorny $1952, p \cdot 380]$

ANTIBYTHOCYPRIS [A. gooberi] Jennings 1936,p.204 [Bairdildae by Jennings] Upper Cretaceous, New Jersey.

ANTICYTHEREIS nom.nov. [Pseudocythereis reticulata Jennings $1936, p \cdot 57, p 1.7, f .10 a-d]$ Bold, van den 1946,p.30 [Cytheridae] Cretaceous, New Jersey. 
ANTIPARAPARCHITES [A.reversus] Coryell \& Rogatz 1932,p.388. [ = Paraparchites Ulrich \& Bassler, by Kellett $1936, p .770$ and by Agnew 1942,p.757, but in the Leperditellidae by Bassler \& Kellett 1934,p.16; Aparchitidae by Schmidt 1941,p.18, and Kloedenellidae-Kloedenellinae by Henningsmoen 1953,p.271] Permian of Texas.

ANTITOMIS [A. bisulcata] Gürich 1896,p.379 [Leperditidae by Gürich; Entomidae by Bassler G Kéllett 1934,p.156] Silurian, Poland.

APARCHITACEA [Superfamily proposed by Swartz 1945b,p.1206] APARCHITINAC Jones 1901, in Chapman 1901,p.147 [of Family Leperditiidae Jones] to include genus Primitia. APARCHITIIDAE Ulrich \& Bassler [n.fam] 1923,p.296 for the genera Aparchites, Leperditella, Schmidtella, Eridoconcha.

APARCHITES [A. whiteavesi] Jones $1889 \mathrm{~b}, \mathrm{p} \cdot 384$ [ieperditidae by Ulrich 1894,p.632; Leperditellidae by Bassler and Kellett 1934,p.14,156; Aparchitidae by Schmidt 1941,p.17 and later workers] Ordovician or early Silurian of Manitoba, Canada. More than 100 species have been reported. 
APARCHITOCYTHERE [A. typica] Swain and Peterson 1952,p.10 [Cytheridae by S.EP.] Upper Jurassic, Wyoming. APATOBOLBINA [A. granifera] Ulrich \& Bassler 1923,p.304 [Primitifdae-Eurychilininae by Bassler \& Kellett 1934,p.162; Beyrichiidae by Swartz 1936; Beyrichiidae-Beyrichiinae by Henningsmoen 1953, p.227,238,269] Silurian, Pennsylvania.

APATOCHILINA [Eurychilina obesa Ulrich 1890,p.129,pl.9,f.13] Ulrich \& Bassler 1923,p.304 [Primitiidae-Eurychilininae by Bassler \& Kellett 1934,p.22; Eurychilininae by Henningsmoen 1953,p.228-267] Ordovician, Kentucky•

APATOCYTHERE [A. simulans] Triebel 1940,p.169 [Cytheridae-Cytherideinae by Triebel] Lower Cretaceous, Germany •

ARCACYTHERE [A. chapmani] Hornibrook 1952,p.31. [Cytheridae-Cytherinae by Hornibrook] Miocene, New Zealand. ARCHICANDONA Bronstein 1939,p.337. Lake Baikal, [ = PSEUDO CANDONA ?]

ARCHICONCHOECIA [A. striata] G. W. Müller 1894,p.225 [Halocypridae-Conchoecinae by Müller 1906a,p.43] Recent, Gulf of Naples. 
ARCHICYTHEREIS [Cythereis yazooensis Howe E Chambers 1934, p.38,pl.1,f.6; pl.6,f.29,30] Howe, in Howe G Law, 1936,p.51. [juvenile of some Cytherid].

ARCYZONA [Amphissites diadematus Van Pelt 1933,p.339,pl.39, f.11,14,15] Kesling 1952a,p.30,31 [Kirkbyidae by Kesling] Devonian, Michigan.

ARDMOREA [A. symmetrica] Bradfield 1935,p.138 [Leperditellidae by Agnew 1942,p.757; Drepane1lidae-Aechmininae by Henningsmoen 1953,p.269] Pennsylvanian, Oklahoma. ARGILLAECIA Brady 1870, Fonds, vol.I,p.198, an error for Argilloecia Sars 1866

ARGILLOECIA [A. cylindrica] Sars 1866,p.17 [Cypridae-Pontocyprinae by Sars 1923,p.53] Recent, Norway.

ARISTOZOE Barrande 1872,p.474-476,pls.22-24,27. "Silurian" Bohemia [ A genus belonging to the Phyllocardia by Kummerow 1931.] Species reported from America under this generic name have been referred to the Conchostraca, genus Aluta by Ulrich E Bassler 1931. The name Aristozoe Barrande with 3 species was reported by Bigsby $1868, \mathrm{p} .200$.

ARTIFACTELIA [A. tomahawki] Coryell \& Booth 1933,p.269 [Bairdiidae by Bassler E Kellett 1934,p.163] Pennsylvanian, Texas. 
ARUNELlA [A. subsala] Brady 1913a,p.232 [Cypridae near Candona by Brady] Recent, England.

ASCIOCYTHERE [Bythocypris rotundus Vanderpool 1928,p.102, pl.]3,f.5,6] Swain 1952,p.75. [Cytheridae by Swain] Lower Cretaceous, Texas.

ASTENOCYPRIS [Leptocypris papracea Sars 1903,p.29,pl.3,f.4, 4a-c] G. W. Müller 1912,p.203,204, nom.nov. for Leptocypris Sars 1903, not Boulenger 1900, Ann. Mus.Congo, Zool. v.1,p.133, Pisc. [Cypridae-Cyprinae by Müller] raised from dried Sumatra mud.

ASTEROPE [A. elliptica] Philippi 1840,p.186-188 [Recent, Mediterranean] Not Asterope Hübner 1816,p.66, nor Asterope Müller E Troschel 1840, fide G. W. Müller 1894,p.216. [ = Cylindroleberis Brady 1868, by Müller $1894, \mathrm{p} .216]$

ASTEROPIDAE Suggested by Brady in Jones and Kirkby 1874, p.4 to include Asterope and Cylindroleberis; Brady \& Norman $1896, p .628$ [ = CYLINDROLEBERIDAE, since Asterope is preoccupied and Cylindroleberis is first available name for the type genus.]

ASTEROPINA nom.nov. Strand 1928,p.40, for Asterope Philippi 1840, nec Hübner 1819 [ = Cylindroleberis Brady 1868] 
ASTEROPINAE G. W. Müller 1912,p.42, subram of Cyprindinidae for Asterope and Cyclasterope.

ASTEROPTERON [Asterope fusca G. W. Müller 1890a,p.242,pl.25, f.11-13; pl.27,f.19-22,25] Skogsberg 1920,p.442 [Asteropidae by Skogsberg = Cylindroleberidae] Recent, Japan.

ATJEHELLA [A. simplicata] Kingma 1948,p.75 [CytheridaeCytherinae by Kingma] Pliocene, Sumatra, Java.

AULACOPSIS [A. bifissurata] Hessland 1949,p.28] [Hollinidae-Ctenentominae by Hessland; Sigmoopsiidae-Glossopsiinae by Henningsmoen 1953,p.202,264] Ordovician, Sweden.

AULOCYTHERIDEA [A. margodentata] Howe 1951b,p.7 [Cytheridae-Cytherideinae by Howe] Eocene, Florida.

AURIGERITES [A. texanus] Roundy 1926,p.6 [Leperditellidae by Bassler \& Kellett 1934,p.163; ?Aparchitidae by Schmidt 1941,p.18; Quasillitidae-Quasillitinae by Henningsmoen 1953,p.275] Mississippian, Texas.

AURIKIRKBYA [Kirkbya wordensis Hamilton 1942 (part) p.713, pl.110,f.13] Sohn 1950,p.35 [Kirkbyidae by Sohn] Permian, Texas. 
AURIS [subgenus of Cythereis] Neviani 1928,p.72,94.

$[=$ Hemicythere Sars 1925] Non Auris Spix 1827, moll., fide Neave 1939,vol.1,p.365.

AVERSOVALVA [Cytheropteron (Aversovalva) aureum] Hornibrook 1952,p.57 [Cytherurinae by Hornibrook] Recent, New Zealand.

AZYGOCYPRIDINA [Crossophorus imperator Brady 1880,p.158, pl.33,f.1-11] Sylvester-Bradley 1950,p.364,nom.nov. , for Crossophorus Brady 1880, not Hemprich and Ehrenberg 1828, a nematode [Cypridinidae by S-B] Recent: S $40^{\circ} 28^{\prime}$ : E. $177^{\circ} 43^{\prime}$.

BAIRDIA [B.curtus, sic.=B. curta] MCoy 1844,p.164 [Bairdiidae by Sars 1888,p.288] Carboniferous, Ireland. [At least 600 species have been referred to this genus: Ordovician? to Recent. The genotype of M'Coy's species was cleaned of matrix and redescribed and figured by Jones and Kirkby 1879, p.567,pl.28,f.I. For most recent study of carapace see Sylvester-Bradley $1950 \mathrm{~b}]$.

BAIRDIACEA Sylvester-Bradley 1948a,p.67, 1948b,p.197; a Superfamily to include families Bairdiidae and Macrocypridae. 
BAIRDIACYPRIS [B. deloi] Bradfield 1935,p.93 [Bairdiidae by Bradfield] Pennsylvanian, Oklahoma.

BAIRDIANELIA [B. elegans] Harlton 1929a,p.160 [Bairdiidae by Harlton] Pennsylvanian, Texas.

BAIRDIIDAE Sars $188^{\circ}, \mathrm{p} .288$.

BAIRDIINAE Sars 1923,p.63, subfamily of Cypridae, for genera Bairdia and Bythocypris.

BAIRDIOCYPRIS [Bythocypris (Bairdiocypris) gerolsteinensis Kegel 1932,p.249,pl.13,f.5] Kegel 1932,p.246. [Bairdiidae by Kegel] Devonian, Germany. (Raised to genus and placed in Healdiidae by Krommelbein $1352, \mathrm{p} \cdot 319-335, \mathrm{pls} \cdot 1-4 \cdot)$

BAIRDIOLITES [B. crescentis] Croneis E Gale 1939,p.288 [Bairdiidae by C. G G.] Mississippian of IIIinois.

BAIRDTTES [B. deltasulcata] Coryell o Malkin 1936,p.9 [Bairdijdae by C. E M.] Devonian, Ontario, Canada.

BAIRDOPPILATA [B. martyni] Coryell, Sample E Jennings 1935, p.2 [BaIrdiidae by C., S. E J.] Upper Oligocene, Mississippi. Range at least Upper Cretaceous to Miocene.

RALANTOIDES [B. quadrilobatus] Morey 1935b,p.478 [Kirkbyidae by Morey; Beyrichiidae by Agnew 1942,p.757; Kloedenellidae-Kloedenellinae by Henningsmoen 1953, 
p.243,245,271.] Mississippian, Wyoming.

BALTICELIA [B. oblonga] Thorslund 1940,p.179. [Kloedenellidae by Thorslund; ?Beyrichiidae by Agnew 1942,p.757; family uncertain by Henningsmoen 1953,p.273] Ordovician, Sweden.

BARYCHIIINA [B. puncto-striata] Ulrich 1891,p.198 [Barychilinidae by Ulrich 1897,p.632] Devonian, Kentucky •

BARYCHILINIDAE Ulrich 1894,p.632 for the genera Barychilina and Kyammodes.

BASSLERATIA [B. typa] Kay 1934,p.34] [Beyrichiidae by Kay; Tetradellidae by Swartz 1936,p.55I; Beyrichiidae-Piritellinae by Kay 1940a,p.265; Drepanellidae-Bassleratiinae by Schmidt 1941,p.50; Tetradellidae-Bassleratiinae by Henningsmoen 1953,p.219-267.] Ordovician, Ontario.

BASSLERATIINAE Schmidt 1941,p.50 [as subfam. of Drepanellidae] to include genera Bassleratia, Thomasatia, Bellornatia, ?Raymondatia, ?Steusloffia, ?Polyzygia.

BASSLERELLA [B. crassa] Kellett 1935,p.155 [Cytheridae by Kellett] Permian of Kansas.

BASSLERELLA Howe $1935, \mathrm{p} \cdot 30[$ [ Basslerites Howe 1937, nom. nov.] 
BASSLERELIA Boucek 1936,p.60 [ = Boucia Agnew 1942, nom.nov.]

BASSLERIA [B. fayettevillensis] Harlton 1929b,p.255

[Aparchitidae by Harlton] Mississippian of

Arkansas. [ $=$ Graphiodactylus by Bassler \& Kellett $1934, \mathrm{p} \cdot 183]$.

BASSLERINA [B. limata] Moore 1929,p.103. Pennsylvanian, Texas. [ Hollinella Coryell, by Bassler and Kellett 1934,p.331, and by Cooper 1946]

BASSLERITES [Basslerella miocenica Howe, in Howe et.al 1935, p.31,pl.1,f.19,24,26] Howe, nom.nov., in Coryell and Fields 1937,p.11. Miocene, Florida.

BASSLERITES [B. hanseni] Teichert $1937 \mathrm{a}, \mathrm{p} .116$ [ Rayella Teichert 1939, nom•nov.

BATHOCYTHERE NOrman 1867,p.198 = misspelling of Bythocythere Sars 1865.

BATTUS Dalman 1826,po151, trilobita, (non Scopoli 1777, Lep.) used by Kloden 1834,p.112-117 for "Battus tuberculatus", which has since been referred to the ostracoda.

BEECHERELLA [B. carinata] Ulrich 1891,p.198 [Beechene]lidae by Ulrich 1894,p.622] Devonian, N.Y. 
BEECHERELIIDAE Ulrich 1894,p.632; Ulrich E Bassler 1923, p.318. Ulrich included genera Beecherella and Krause1la.

BELLORNATIA [B. tricollis] Kay 1934,p.342 [Beyrichiidae by Kay 1934; Tetradellidae by Swartz 1936,p.551; Beyrichiidae-Piritellinae by Kay 1940a,p.265; Drepanellidae-Bassleratiinae by Schmidt 1941,p.50; Tetradellidae-Bassleratiinae by Henningsmoen 1953, p.219,267.] Ordovician, Iowa.

BERNIX [Beyrichia tatei Jones 1864,p.87-89,fig.3] Jones 1885a,p.319. [Kloedenellidae by Bassler \& Kellett 1934,p.30; family uncertain by Henningsmoen 1953, p.273.] Carboniferous, England.

BEROUNELLA [B. rostrata] Boucek 1936,p.65 [Beyrichiidae by Boucek; ?Beecherellidae by Agnew 1942,p.757;

Alanellidae by Henningsmoen 1953,p.273]. Silurian, Bohemia.

BERTIILONELIA [B. subcircularis] Stewart and Henrix 1945b, p.100 [Leperditellidae by SEH] Devonian, Ohio.

BEYRICHIA [B. kloedeni] MPCOy 1846,p.57 [Leperditiidae-Beyrichiinae by Jones 1901,p.147; Beyrichildae-Beyrichiinae by Henningsmoen 1953, p.236,238,269.] Silurian, Ireland. 
BEYRICHIA [Battus tuberculatus Kloden 1834,p.115-117,pl.1, f.21-23] Boll 1847,p.127, Silurian drift, Germany.

BEYRICHIA M'Coy, of Jones 1855,p.85 is divided into groups: Simplices, Corrugatae, and Jugosae.

BEYRICHIACEA Ulrich and Bassler 1923,p.297 [Superfamily to include Primitiidae, Zygobolbidae, Beyrichildae, Kloedenellidae, Kirkbyidae]. Revised by Swartz $1936, p \cdot 556-557$; and by Henningsmoen 1953.

BEYRICHIANA [B. permiana] Kellett 1933, p.73 [Beyrichiidae by Kellett; Hollinidae? by Swartz 1936,p. 149; Kloedenellidae-?Kloedenellinae by Henningsmoen 1953,p.244,271] Permian, Kansas.

BEYRICHIELLA [B. cristata] Jones and Kirkby 1886a,p.438 [Beyrichiidae by Ulrich 1894,p.632; Kloedenellidae by Bassler G Kellett 1934,p.211; Kloedenellidae-Kloedenellinae by Henningsmoen 1953,p.243,271]. Carboniferous, Scotland.

BEYRICHIIDA Pokorný 1953,p.221,227,230, an Order of the Class Ostracoda, in which Pokorný places the Beyrichiacea of Ulrich and Bassler 1923, and the family Aparchitidae Jones 1901. 
BEYRICHIIDAE Ulrich 1894,p.632; Ulrich and Bassler 1923, p.310; Swartz 1936,p.548; Schmidt 1941,p.24; Henningsmoen 1953,p.197,235,269. A very large family as first used by Ulrich, but since reduced.

BEYRICHIINAE Jones, nov., in Chapman 1901,p.147, to include the genera Kloedenia, Bollia and Beyrichia.

BEYRICHINAE Matthew $1886, p .63$, a subfamily of Ostracoda to include "Beyrichiae jugosae Jones 1855 = Beyrichia (part), "Beyrichiae unguiformes" = Hipponicharion, n.gen., "Beyrichiae corrugata" Jones $1855=$ Beyrichia (part), and "Beyrichiae altiformes," = Beyrichona, n.gen.

BEYRICHIOPSIINAE Henningsmoen 1953,p.244, a subfamily of Kloedenellidae for Beyrichiopsis, Deloia, Denisonella.

BEYRICHIOPSIS [B. fimbriata] Jones and Kirkby $1886 \mathrm{a}, \mathrm{p} .434$ [Beyrichiidae by Ulrich 1894,p.632; Kloedenellidae by Ulrich \& Bassler 1923,p.213; KloedenellidaeBeyrichiopsiinae by Henningsmoen 1953,p.272.] Carboniferous, Scotland.

BEYRICHONA Natthew 1886,p.64,65, Cambrian, New Brunswick [Not Ostracoda, but Conchostraca, by Ulrich \& Bassler $1931, p \cdot 42$. 
BICORNELIA [B. tricornis] Coryell and Cuskley 1934,p.3

[Primitiidae by CEC; Kloedenellidae-?Kloedenellinae by Henningsmoen 1953,p.244,271.] Devonian, Oklahoma.

BICORNELIINA [B. bolchovitinovae] Zaspelova 1953,p.183

[Drepanellidae-Neodrepanellinae by Zaspelova] Denovian, USSR.

BIFLABELLUM [B. tencrum] Öpik 1935c,p.86, 1937,p.102]

Ordovician, Estonia. [Not Biflabel]um Doederlein 1913; = Opikium Agnew 1942,p.757,759, new name.]

BINODELLA [B. binoda] Bradfield 1935,p.140 [Kirkbyidae by Bradfield] Pennsylvanian, Oklahoma.

BIRDSALlELlA [B. simplex] Coryell and Booth 1933,p.271 Cytherellidae by CEB.] Pennsylvanian, Texas.

BISACCULUS [B. bilobus] Stewart and Hendrix 1945b, p.103. [Kirkbyidae by SEH.; Hollinidae by Henningsmoen 1953,p.270. Devonian, Ohio.

BOLBIBOLIIA [B. labrosa] Ulrich and Bassler 1923,p.299,301 [Primitiidae by UEB; Beyrichiidae-Beyrichiinae by Henningsmoen 1953,p.238,269.] Silurian, Island of Anticosti.

BOLBINA [Bollia ornata Krause 1896, of Bonnema 1909,pl.4,f.4] Henningsmoen 1953,p.208 [Sigmoopsiidae-Sigmoopsiinae by Henningsmoen] Ordovician drift boulder in North Germany. 
BOLBIPRIMITIA [Halliella fissurella Ulrich \& Bassler 1923b, p.514,pl.37,f.22,23] Kay 1940a,p.234,240.

[Primitildae by Kay; Beyrichildae-Beyrichiinae by Henningsmoen 1953,p.269] Silurian, W. Va.

BOLBOZOE [B. anomala, B.bohemica, B. jonesi; B. anomala designated by Bassler \& Kellett 1934,p.215; B. bohemica designated by Bouček 1936,p.62] Barrande 1872,p.500. [Bolbozoidae by Bouček 1936,p.62] Silurian, Bohemia. ["Not considered an ostracod" by Bassler \& Kellett 1934,p.215] [These are ostracods: Entomozoacea-Bolbozoidae by Pŕibyl 1951, p.104]

BOLBOZOIDAE Bouček 1936,p.62 ["known not to be ostracodes" by Agnew 1942,p.756, but does not indicate proper place] For the genus Bolbozoe. [These are ostracods and placed in Entomozoacea by Pribyl $1951, \mathrm{p} \cdot 104]$

BOLIIA [B. uniflexa Jones \& Holl, designated by Miller 1892, p.706, fide Warthin 1948,p.646] Jones and Holl $1886 \mathrm{a}, \mathrm{p} .360$ [Beyrichiidae by Ulrich 1894,p.632; Leperditiidae-Beyrichiinae by Jones 1901,p.147; Primitiidae-Primitiinae by Bassler \& Kellett 1934, p.17; Drepanellidae by Swartz 1936,p.553; Drepanellidae-?Bolliinae by Henningsmoen 1953,p.209, 232,233,268] Silurian, England. 
BOLIIINAE Bouček 1936,p.40; subfam of Primitildae Ulrich and Bassler 1923, for the genera Bollia, Placentula, Jonesella, Bolbibollia.

BONNEMAIA [B. celsa] Ulrich and Bassler 1923,p.305

[Zygobolbidae-Zygobolbinae by UEB; Beyrichiidae-Zygobolbinae by Henningsmoen 1953,p.238,269; Silurian, Maryland.

BosquetiA [B. robusta] Brady, Crosskey, and Robertson 1874, p.220 [?Cytherellidae by B,C.ER] Pleistocene, Scotland.

BOUČEKITES [B. devonica] Pribyl (1951) 1953,p.31-34. [Incertae familiae (Aparchitidae or Acronotellidae? by Bouček; ?Alanellidae by Henningsmoen 1953 p.248,273] Devonian, Bohemia.

BOUCIA [Basslerella ornatissima Boucek 1936,p.61,pl.6,f.1, text figs.3a,b] Agnew [nom.nov.] 1942,p.757 [? Entomidae by Agnew; Entomozoacea-Entomozoidae-Bouciinae by Pribyl 1951,p.106] Silurian, Bohemia.

BOUCIINAE Pribyl 1951,p.106, a subfamily of Entomozoidae, for the genus Boucia.

BOURSELLA [B. trilobita] Turner 1939,p.13 [Beyrichiidae by Turner; Kloedenellidae-Kloedenellinae by Henningsmoen 1953,p.244,271; Drepanellidae by Kesling 1953a, p.197] Devonian, Ontario. 
BRACHYCYTHERE [Cythere sphenoides Reuss 1854,p.141,pl.27,

f.2] Alexander 1933,p.204 [Cytheridae by

Alexander] Upper Cretaceous, Austria. Range:

Jurassic to Recent.

BRADLEYA [Cythere arata Brady 1880,p.101,pl.24,f.2a-c]

Hornibrook 1952,p.38. Cytheridae-Trachyleberinae by Hornibrook] Recent, South Pacific.

BRADORIA [B. scrutator (part), Matthew 1899,p.204,pl.4,f.la-c, by Ulrich \& Bassler] Matthew 1899,p.204. Cambrian, Nova Scotia. [Not Ostracoda, but Conchostraca by Ulrich \& Bassler 1931,p.12]

BRADORONA [B. perspicator] Matthew 1902,p.444, Cambrian, Nova Scotia. [ = Bradoria Matthew, by Ulrich \& Bassler 1931,p.12]

BRADYCINETUS [Cypridina globosa Lilljeborg 1853,p.171,pl.17, f.2-10;pl.18,f.1-3,7] Sars 1866,p.109. Recent, Scandinavia. [ = Philomedes Lilljeborg 1853, by Sars 1922,p.12.]

BRADYCYPRIS [Cypris intumescens Brady 1907a,p.173,pl.29, f.1-5] Sars 1924a,p.145. [Cypridae by Sars] Recent, Natal.

BRIARTINA [Leperditia quenstedti Gümbel 1874,p.69] Kegel 1933,p.924 [as subgenus of Leperditia; Leperditiidae-Leperditiinae by Henningsmoen 1953,p.274] Devonian, Germany. 
BRILLIUS [B. distortus] Brayer 1952,p.171 [Kirkbyidae by Brayer] Mississippian of Missouri.

BROMIDELLA [B. reticulata] Harris $1931, p .93$ [Primitiidae-Eurychilinae by Bassler \& Kellett 1934,p.20,2l, 223; Beyrichiidae-Beyrichiinae by Kay 1940a, p.263; Hollinidae-Follininae by Schmidt 1941, p.32; Primitiidae by Henningsmoen 1953,p.226,267] Ordovician, Oklahora.

BUDAIA [B. prima] Méhes 1941a,p.67 [Cytheridae by Mehes] Oligocene near Budapest, Hungary. [non Budaia Wells 1933, coel., fide Neave 1939,vol.1]

BUDNIANELLA [B. caroli] Bouček 1936,p.69 [Kirkbyidae by BouKek; Incertae sedis by Agnew 1942,p.757;

Beyrichiacea but fam uncertain by Henningsmoen 1953,p.273] Silurian, Bohemia.

BUFINA [B. elata] Coryel1 and Malkin 1936,p.8 [Ropolonellidae by CEM.; Quasillitidae-Quasillitinae by Henningsmoen 1953,p.256,257,275] Devonian, Ontario.

BUNTONIA [B. shubutaensis] Howe, in Howe and Chambers 1935, p.22 [Cytheridae by Howe] Eocene, Mississippi.

BURIELIA [B. pecanata] Coryel1 and Booth 1933,p.268 [Bairdiidae by CEB] Pennsylvanian of Texas. 
BURSULLELA [B. triangularis] Jones 1887,p.7; 1888a,p.409, pl.22,f.5,6. [Primitiidae by Bassler \& Kellett 1934,p.223; Leperditellidae by Swartz 1936,p.550; ?Aparchitidae by Schmidt 1941;p.18.] Silurian, Island of Gotland.

BYTHOCERATINA [B. mestayerae] Hornibrook 1952,p.62 [Cytheridae-Bythocytherinae by Hornibrook] Recent, New Zealand.

BYTHOCYPRIS [Bairdia bosquetiana Brady 1866,p.364,pl.57= the young of Bythocypris reniformis Brady 1880 , p.46,pl.5,f.la-1] Brady 1880,p.45 [Cypridae by Brady; Nesideidae by Muller 1912,p.249; Bairdiinae by Sars 1923,p.63; Bairdiidae by most subsequent authorsl Recent, Atlantic Ocean.

BYTHOCYPROIDEA [B. sandyskyensis] Stewart and Hendrix 1945a, p.91 [Bairdiidae by SEH.] Devonian, Ohio.

BYTHOCYTHERE [B. turgida Sars 1866, designated by Brady and Norman 1889,p.220] Sars 1866,p.82 [Cytheridae-Bythocytherinae by Sars 1926,p.232-233] Recent, North Atlantic. For shell structure see Sylvester-Bradley $1948 \mathrm{c}$.

BYTHOCYTHERINAE Sars 1926,p.232-233, subfamily of Cytheridae, for genera Bythocythere, Pseudocythere, Macrocythere. 
CALLIZOE [C. bohemica] Barrande 1872, p.503 [see also Callizoe bohemica Barrande in Bigsby 1868,p.200, name only] "Silurian," Bohemia. [ = a Leperditoid Phyllopod by Jones $1883, p .463]$

CAMDENIDEA [C. camdenensis] Swain 1953,p.279 [Cypridae by Swain] Devonian, Tennessee.

CAMPTOCYTHERE [C. praecox] Tricbe] 1950a,p.198,199

[Cytheridae-Loxoconchinae by Triebel] Jurassic, Germany •

CAMPYLOCYTHERE [C. laeva] Edwards 1944,p.514 [Cytheridae-Cytherideinae by Edwards] Miocene, North Carolina.

CANDOCYPRIA [C. osburni] Furtos 1933,p.458 [Cypridae-Candocyprinae by Furtos] Recent, Ohio.

CANDOCYPRINAE Alm 1915,p.31-33 Subfam. of Cypridac; Furtos, 1933,p.457,458; Furtos 1936,p.112; Klie 1938a, p.25. [Proposed to include Candoninae $t$ Cyclocyprinae of previous authors]

CANDONA [Cypris reptans Baird 1835,p.99,pl.3,f.11 was designated by Baird $1846, p .414$, from original list which consisted of C. candida (O.F. Müller), C. reptans" (Baird), C. hispida (Baird), C. detecta 
(O. F. Müller), and Candona similis n.sp. nude name] Baird 1845,p.152,153. [Cypridae by Baird] Recent, British Isles. [Note: $\underline{C}$. candida was later designated by Brady and Norman, 1889, p.98 as genotype, and $\underline{C}$. reptans was made their type of the new genus Erpetocypris. BEN 1889,p.84. These authors had obviously overlooked Baird's 1846 designation.] Recent, British Isles.

CANDONELIA [Candona brachyura Heller 1871,p.93,pl.2,f.1-8] Claus 1891b,p.231 in footnote [Genus and species uncertain, perhaps Potamocypris by G. W. Müller 1912,p.222] Recent, Europe.

CANDONELIA Claus, of Vávra 1898a,p.12-16, used as a subgenus of Cypridopsis for 8 species, 6 of which were referred to Cypridopsis by G. W. Müller 1912, p.211-213; the others to Potamocypris by Müller $1912, p \cdot 216$.

CANDONIDES Sars 1923,p.71 a group of Cypridae-Cyprinae for Candona, Cryptocandona, and Candonopsis.

CANDONINAE Kaufmann 1900a,p.107-108; Müller 1900,p.12,13; Alm 1915,p.105-107; subfam. of the Cypridae. Kaufmann included the genera Candona, Typhlocypris, Candonopsis, Paracandona, Cryptocandona. 
CANDONINAE Daday 1900,p.205, a subfam. of Cypridae, with subtribes Euopsida Zygopsida, Synopsida, Typhlopsida, for the genera Notodromas, Cyclocypris, Cypria, Iliocypris, Iliocyprella, Candonopsis, Eucandona, Candona, and Typhlocypris.

CANDONINI Bronstein 1947,p.187-266, a Tribe of Cypridae-Candoninae for the genera Pseudocandona, Candona, Cryptocandona, Paracandona, Candonopsis.

CANDONOCYPRIS [C. candonoides (King)=Cypris candonoides King 1855,p.66,pl.10,fig.F; and C. assimilis n.sp.; the first should be genotype] Sars 1894,p.34. [Herpetocypridinae by Henry 1923,p.280] Recent, Australia.

CANDONOPSIS [Candona kingsleyi Brady and Robertson 1870, p.17,pl.9,f.9,10 (not figs.11,12] Vávra 1891a, p.161; vávra 1891b,p.54 [Cypridae by vávra; Cyprididae-Candoninae by Kaufman 1900a,p.107] Recent, England.

CANDONOPSIS [C. pyriformis] Almeida 1950,p.33 [Cypridae by Almeida] Liassic of Brazil. A junior homonym.

CARBONIA [C. agnes] Jones 1870d,p.218 [Cytheridae by Ulrich 1894,p.632; Kirkbyidae by Bassler \& Kellett 1934, p.237] Coal Measures, South Wales. [Preoccupied by Carbonia Robineau-Desvoidy 1863; = Carbonita Strand, new name, (1926) 1928,p.41.] 
CARBONITA Strand (1926) 1928,p.41 [new name for Carbonia Jones.]

CARBOPRIMITIA [C. depressa] Croneis and Funkhouser 1939, p.337 [Primitiidae by CGF; Kloedenellidae-Kloedenellinae by Henningsmoen 1953,p.243,271.] Mississippian or Illinois.

CARDINIFERELIA [C. bowsheri] Sohn 1953,p.66 [Cardiniferellidae by Sohn] Carboniferous, Texas.

CARDINIFERELIIDAE Sohn 1953,p.66, new family for single genus

\section{Cardiniferella.}

CARBINOBOLBINA [Ctenobolbina carinata estona Öpik 1937,p.98, pl.8,f.8, = Carinobolbina estona Opik by Henningsmoen] Henningsmoen 1953,p. 205, text fig.5. [Sigmoopsiidae-Sigmoopsiinae by Henningsmoen] Ordovician, Esthonia.

CARNARVONOPSIS Swartz 1945i,p.1205. [Leperditiidae by Swartz.] Genotype not given.

CARYON [C. bohemicum] Barrande 1872,p.505 [see also Caryon Barrande in Bigsby 1668,p.192, in list] Upper Silurian by Barrande.

CATIVELLA [C. navis] Coryell and Fields 1937,p.9. [Cytheridae-Cytherinae by CEF.] Miocene, Panama. 
CAUDITES [C. medialis] Coryell and Fields 1937,p.10

[Cytheridae-Cytherinae by CEF.] Miocene, Panama.

CAVELIINA [C. pulchella] Coryell 1928a,p.89,90 [Cytherellidae by Coryell; Healdiidae by Triebel 1950b, p.116] Pennsylvanian, Oklahoma. [non Cavellina (pro Clavelina Savigny 1816) Maitland 1897,p.34, fide Neave 1939, vol.1, p.611]

CAVELLINELLA [C. casei] Bradfield 1935,p.136 [Cytherellidae by Bradfield] Pennsylvanian, Oklahoma. $[=a$ juvenile Cavellina by Cooper 1946,p.72]

CAVELLININAE, Egerov 1950, by Polenova 1952,p.106, subfamily of Kloedenellidae. Polenova mentions here: Donellina, Semilukiella, Sulcocavellina, Cavellina, and gives range Silurian? to Permian.

CELECHOVItes [C. cultratus] Pokorný 1950,p.562,623 [Podocopa Incertae Sedis by Pokorný] Devonian, Czechoslovakia.

CENTROCYPRIS [Cypris (Centrocypris) horrida] Vávra 1895, p.15 [Cypridae-Cyprinae by G. W. Müller 1912, p.160] Recent, Zanzibar.

CERATELIA U1rich 1890,p.113. non Gray 1869, Coel. [nom.nud. = Ceratopsis Ulrich $1894, \mathrm{p} .675$ by Bassler and Kellett 1934,p.242] 
CERATOCYPRIS [C. symmetrica] Poulsen 1934,p.38 [Bairdiidae by Poulsen; Aparchitidae by Hessland 1949, p.148; ?Leperditellidae-?Conchoprimitiinae by Henningsmoen 1953,p.255,275] Silurian of Greeland.

CERATOPLEURINA [C. mimiri] Coryell and Johnson 1939,p.221 [Glyptopleuridae by CEJ.] Mississippian of Illinois. [ = Glyptopleura Girty, by Cooper 194I, p.42]

CERATOPSIS [Beyrichia chambersi Miller 1874,p.234,fig.27] Ulrich 1894,p.675 [Beyrichiidae by Bassler $\varepsilon$ Kellett 1934,p.242; Tetradellidae by Swartz 1936,p.551; Primitiidae-Dilobellinae by Kay 1940a, p.257; Hollinidae-Tetradellinae by Hessland 1949, p.329; Tetradellidae-Tetradellinae by Henningsmoen 1953,p.216,267.] Ordovician, Ohio.

CHESTERELIA [C. fissurata] Croneis and Gutke 1939,p.45 [Kloedenellidae-Kloedenellinae by Henningsmoen 1953,p.243,271] Mississippian of Illinois.

CHEVROLEPERDITIA [C. chevronalis] Swartz 1949,p.319

[Leperditiidae-Leperditiinae by Swartz] Ordovician or Silurian in well, Early County, Georgia.

CHILOBOLBA Bonnema 1938,p.104 = Chilobolbina. 
CHILOBOLBINA [Primitia dentifera Bonnema 1909,p.25,pl.2, f.1-5] Ulrich and Bassler 1923,p.304 [Primitiidae-Eurychilininae by UEB.; Beyrichiidae by Swartz 1936,p.548; Primitiidae by Henningsmoen 1953, p.225,227,267] Ordovician, Esthonia.

CHIRONIPTRUM [C. oiostathmicum] Kesling 1952a,p.36 [Kirkbyidae by Kesling] Devonian, Michigan.

CIADOCOPA Sars 1866,p.121, a Suborder for Family Polycopidae, with single genus Polycope.

CHLAMYDOTHECA [Cypris (Chlamydotheca) azteca] Saussure 1858 , p.487-490 [Cypridae-Herpetocypridinae by Sharpe 1903,p.975; Cypridae-Cyprinae by Furtos 1933, p.438] Recent, Mexico.

CLITHOCYTHERIDEA Van den Bold 1946,p.24,80-82 = Clithrocytheridea Stephenson.

CIITHROCYTHERIDEA [Cytheridea (?) garretti Howe and Cambers $1935, \mathrm{p} \cdot 14, \mathrm{pl} \cdot 1, f \cdot 4,5 ; \mathrm{pl} \cdot 2, f .11,12 ; \mathrm{pl} \cdot 6, f \cdot 10,11]$ Stephenson 1936,p.702; 1946a,p.326 [Cytheridae-Cytherideinae by Van den Bold 1946,p.24] Eocene, Alabama.

CNESTCCYTHERE [C. lameIIicosta Triebel, nom.nov., for Cythere reussi Procházka 1893,p.79,pl.2,f.la,b; not Cythere reussi Brady 1869, Fonds, vol.1.p.153,pl.18,f.9-10] 
Triebel 1950,p.316. [Cytheridae-Cytherinae by Triebel] Miocene, Vienna Basin.

CODONOCERA [C. cruenta] Brady 1902,p.188 [Cypridinidae by Brady] Recent, Malay Archipelago.

COELOCHILINA [Eurychilina aequalis Ulrich 1890,p.129,pl.9, f.5-8] Ulrich and Bassler 1923,p.303. [Primitiidae-Eurychilininae by UEB; Beyrichiidae by Swartz 1936,p.548; Eurychilinidae-Eurychilininae by Henningsmoen 1953,p.228,267] Ordovician, Kentucky•

COELONELIA [Isochilina? scapha stewart 1930,p.57,pl.1,f.1], 12] Stewart 1936,p.742 [Leperditellidae by Stewart; Kloedenellidae-Kloedenellinae by Henningsmoen 1953,p.271] Devonian, Ohio.

COELOENELIINA [C. parva] Polenova 1952,p.66 [Leperditellidae by Polenova] Denovian, USSR.

COLPOS [C. insignis] Moberg 1895,p.12 [Ostracode? by Bassler and Kellett 1934,p.247] Silurian, Sweden.

CONCHAECIA [C. agilis, C. rostrata, C. brevirostris, C. inflata] Dana 1849,p.51-53 [Halocypridae-Halocyprinae by Dana 1653,p.1281; Conchoecidae by Sars 1866,p.114; Conchoecinae by Claus 1891a,p.55; Halocypridac-Halocyprinae by Granata and Caporiacco 1949,p.10,16] Recent, Pacific Ocean. 
CONCHOECETTA [C. acuminata Claus [1890] 1891,p.16; 1819a, p.67,pls.13,14] Claus [1890] 1891,p.16; 1891a, p.67 [Halocypridae-Conchoecinae by Claus; = Conchoecia by G. W. Müller 1906a,p.76; 1912,p.74; Halocypridae-Halocyprinae by Granata and Caporiacco 1949,p.15,21] Recent, Atlantic Ocean.

CONCHOECIA Dana 1853,p.1281,1298, and later authors = Conchaecia Dana 1849.

CONCHOECIADAE Jones $1873 a, \mathrm{p} .73$ = Conchoecidae of Sars 1866. CONCHOECIDAE Sars $1866, p .114$ = Halocypridae-Halocyprinae of Dana and others, for genus Conchoecia.

CONCHOECILIA [C. daphnoides Claus [1890] 1891,p.18; 1891a, p.68,pl.15] Claus [1890] 1891,p.17; 1891a,p.68 [Halocypridae-Conchoecinae by Claus; = Conchoecia by G. W. Müller 1901a,p.6; 1906a,p.126; 1912,p.94; Halocypridae-Halocyprinae by Granata and Caporiacco 1949,p.10,15] Recent, Atlantic.

COPECHAETE [C. elongata, C. affinis, C. fissa, C. armoricana] Hesse 1878,p.18,19["Famille des Copechetiens" by Hesse,p.19] Recent, France. [ = Cylindroleberis Brady 1868]

COPÉCHETIENS, Famille des, Hesse $1878, \mathrm{p} .19$ = Cylindroleberidae 
Copytus [C. caligula] Skogsberg 1939,p.418 [Cytheridae near Cytherideis by Skogsberg] Recent, South Georgia.

CORNIA Lutkevich $1937,0.63$ [Iisted as an ostracod by Agnew 1944,p.218; but described as a Phyllopod; = Conchonstraca by Branson 1948, p.846] Permian, Siberia and USSR.

CORNIGELLA [C. minuta] Warthin 1930,p.59 [Beyrichiidae by Warthin; ?Kirkbyidae by Swartz 1936,p.549; Drepanellidae by Kellett 1936,p.771; Kloedenellidae-Kloedenellinae by Henningsmoen 1953, p.271] Pennsylvanian, Oklahoma. [Note: Cooper 1946,

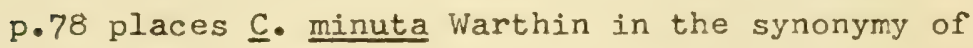
C. tuberculospinosa (Jones \& Kirkby), a British species].

CORNULINA [C. bispinosa] Coryell and Williamson 1936, p.2 [Primitiidae by CEW; Drepanellidae-Drepanellinae by Schmidt 1941,p.50] Silurian, Indiana. [Not Cornulina Conrad 1853; = Waldronites Coryell and Williamson nom.nov. in Agnew 1942,p.760.]

CORRUGATAE Jones 1855a, p.85, a Group of species of the genus Beyrichia $\mathrm{M}^{\prime} \mathrm{Coy}$.

CORYELLA [C. stovalli] Harris and Lalicker 1932,p.397

[Primitiidae by HEL.] Carboniferous, Kansas.

$[=$ Jonesina bolliaformis (Ulrich \& Bassler) by 
Bassler \& Kellett 19;4, p.345; = Knoxina bolliaformis (UEB) by Kellett 1943,p.620].

CORYELIINA [C. capax] Bradfield 1935,p.35 [Primitiidae by Bradfield] Pennsylvanian, Oklahoma.

CORYELLINA [C firma] Kellett 1935,p.138 [Bairdiidae by Kellett] Pennsylvanian, Kansas [Not Coryellina Bradfield, Jan. 1935 = Coryellites Kellett 1936, nom.nov.]

CORYELITES [Coryellina firma Kellett 1935] Kellett 1936, p.775, new name for Coryellina Kellett, not Bradfield. [Note: Cooper 1946,p.57 has placed Bythocypris angularis, $\underline{B} \cdot$ deesensis, $\underline{B} \cdot$ hoxbarana Bradfield 1.935, and B. pediformis Warthin 1930 in the synonymy of Coryellina firma. All of these species have time preference.]

COSTA [Cytherina edwardsi Roemer 1838; Cythereis antiquata Baird 1850; Cythere polytrema Brady 1878; Cythereis laticapitata Neviani 1928; Cythereis subcrispa Neviani 1928; "Cythere batei Brady 1865" = Cythereis batei Brady 1866 cited] Neviani 1928,p.72,76 [as "Gruppo" = subgenus or genus - of "Cythereis"] This "Gruppo" certainly does not correspond to Trachyleberis as suggested by Ruggieri 1950a,p.15, and the genotype should be Cytherina edwardsi 
Roemer, which was described from Palermo. For

a modern figure of Cythere edwardsi, see Ruggieri 1950a, p.16, figure 4.

CostatiA [Costatia posneri Polenova,n.sp] Polenova 1952, p.109 [Quasillitidae by Polenova] Mid Devonian, USSR.

CRASPEDOBOLBINA [C. dietrichi] Kummerow 1924,p.427

[Primitiidae-Eurychilininae by Kummerow; Beyrichiidae-Zygobolbinae by Henningsmoen 1953,p.227, 239,270] Ordovician drift, Germany.

CRATERELIINA [C. robusta] UIrich and Bassler 1913a,p.539, 540 [Thlipsuridae by UEB] Devonian, Pennsylvania. [Craterellina robusta $=$ ThIipsura robusta by Swartz 1932,p.39,42,43, and by Bassler and Kellett $1934, p .484$, and the genus was abandoned because its type species did not have the characters it was supposed to possess. Eucraterellina Wilson 1935, 0.640 was described with a genotype which possessed the characters $\underline{C}$. robusta was supposed to have. Henningsmoen $1953, \mathrm{p} .260$ has retained both Eenera in the Thlipsuridae.]

CRESCENTJLIA [C. pugnax] Barrande 1872,p.507, [Drepanellidae-Aechmininae by Schmidt 1941,P.63; Drepanellidae-?Bollinae by Henningsmoen 1953,p.234,268\} "Silurian" [Ordovician, ] Bohemia. 
CRIBROCONCHA [C. costata] Cooper 1941,p.29 [Bairdiidae by Cooper; ?Healdiidae by Henningsmoen 1953,p.260] Mississippian of Illinois.

CROSSOPHORUS [C. imperator] Brady 1880,p.157 [Cypridinidae by Brady] Recent, near New Zealand. [Not Crossophorus Hemprich and Ehrenberg 1828, a nematode = Azygocypridina Sylvester-Bradley 1950,p.364, new name.

CRYPTOCANDONA [C. vávrai] Kaufmann 1900c,p.132 [Cypridae-Candoninae by Müller 1912,p.148] Recent, Switzerland.

CRYPTOCARIS [8 species, one of which C? rhomboidea, $\mathrm{p} .464$, has been made genotype of Rhomboentomozoe] Barrande $1872, p .459$ [Described as a genus of Phyllopoda by Barrande].

CRYPTOPHYLLUS [C. sulcatus] Levinson 1951,p.558 [Beyrichiaceae-Primitidae by Levinson; ?Leperditellidae-?Eridoconchinae by Henningsmoen 1953,p.256,275] Ordovician, Indiana.

CTENENTONINAE Schmidt 1941,p.34, subfamily of Hollinidae to include Ctenentoma, Winchellatia, Parabolbina, Acronotella, ?Hippa, and ?Eoconchoecia. [Henningsmoen $1953, p .224,225,267$ indicates this subfamily is 
invalid, as Ctenentoma is a single internal mold of an indeterminate Steuslorfia, and at best it is younger and a synonym of Bassleratiinae.

CTENENTOMA [Entomis umbonata Steusloff 1894,p.778,p1.58, f.20] Schmidt 1941,p.35 [Hollinidae-Ctenentominae by Schmidt; invalid = internal mold of some Steusloffia, perhaps $\underline{S}$. polynodulifera or $S$. lineata (Krause) by Henningsmoen 1953,p.224,267] Ordovician drift, Germany.

CTENOBOLBINA [Beyrichia ciliata Emmons 1855,p.219, text fig.74c] Ulrich 1890,p.108 [Beyrichiidae by Ulrich, Hollinidae-Tetradellinae by Schmidt 1941, p.3.7; Sigmoopsildae-Sigmoopsiinae by Henningsmoen 1953,p.211,239,266] Ordovician, Ohio.

CTENOBOLBINELLA [C. carinata] Kummerow 1953,p.39 [Beyrichiidae by Kummerow] Devonian, Germany. CTENOCYPRINA Daday 1900,p.125,304, a Tribe of subfamily Cyprinae, with subtribes Zygopsida and Synopsida, for genera: Centrocypris, Cypris, Eucypris, Herpetocypris, Cyprois, Cypricercus.

CTENOLOCULINA [Tetradella cicatricosa Warthin 1934,p.209, pl.1,f.4-6] Bassler 1941,p.22 [Tetradellidae by Bassler; Hollinidae by Pokorný 1950,p.526, by 
Kesling 1952,p.46, and by Henningsmoen 1953, p.270] Devonian, Michigan.

CTENONOTELLINAE Schmidt 1941,p.48 - Subfamily of Hollinidae, for Ctenotella, Dicranella, Rakervella. [Included in Piretellinae of Opik by Henningsmoen 1953, p.197,217]

CTENONOTELLA [C. elongata] Öpik 1937,p.101 [Hollinidae-Ctenonotellinae by Schmidt 1941,p.48; Tetradellidae-?Piretellinae by Henningsmoen 1953,p.217,219,267] Ordovician, Estonia.

CUNEOCYTHERE [C. truncata] Lienenklaus 1894,p.259 [Cytheridae-Cytherideinae by Van den Bold 1946, p.26] Oligocene, Germany •

CUSELINA [C. impressa] Ammon 1910, p. 53 [Ostracoda, incertae sedis by Güthorl 1934,p.11,pl.1,f.2 (Lectotypus)] Permian, Germany.

CUSHMANIDEA [Cytheridea seminuda Cushman 1906,p.374,pl.33, f.62-64; pl.34,f.76,77] Blake 1933,p.232-233 [Cytheridae-Cytherideinae by Blake] Recent, Maine.

CYAMOCYPRIS [Cypris valdensis Fitton 1836,p.177; Sowerby 1836, p.344,pl.21,f.1] Anderson 1939,p.305 [Cypridae-Rostocyprinae by Anderson] Jurassic, England. [ Cyamocypris $=$ subgenus of Cypridea by Sylvester-Bradley $1949, \mathrm{p} .127$. 
CYATHUS [C. ulrichi] Roth and Skinner 1930,p.347 [Aparchitidae by RES; Leperditellidae by Bassler \& Kellett 1934, p.255; Beyrichiacea of uncertain family by Henningsmoen 1953,p.273] Pennsylvanian, Colorado.

CYCLAS [Used by Eichwald 1857,p.307 thus: Cytherina (Cyclas) eos; Permian, Russia.] Not Cyclas Lamarck (1798) Ency. Meth. (Tabl. vers) pl.301; 1799, mem.soc. hist. nat., Paris, 84, Moll. by Neave 1939, vol.1, p.914.

CYCLASTEROPE [C. hendersoni,n.sp., and C. orbicularis, n.sp.] Brady 1897, p.85 [Cypridinidae by Brady; Asteropidae by Skogsberg $1920, p \cdot 542$ - who designated $\underline{C}$. hendersoni genotype] Recent, Madras Harbour.

CYCLOCYPRIA [C. kincaidia] Dobbin 1941,p.235 ["intermediate form between Cyclocypris and Cypria" by Dobbin] Recent, Washington.

CYCIOCYPRIDES Sars 1925,p.89, a Group of Cypridac-Cyprinae for Cyclocypris and Cypria.

CYCLOCYPRIDINAE Kaufmann 1900a,p.107; 1900b, p.319-321, Subfamily of Cyprididae to include: Cyclocypris, Cypria, and Physocypria.

CYCIOCYPRINAE lioff 1942,p.100,101, change in spelling of Cyclocypridinae. 
CYCLOCYPRINI Bronstein 1947,p.175-186, a Tribe of Cypridae-Cyprinae, for Cyclocypris, Cypria, Physocypria.

CYCLOCYPRIS [Cypris globosa Sars 1863,p.27] Brady and Norman 1889,p.70. [Cypridae-Cyclocypridinae by Kaufmann 1900a,p.107; Cypridae-Cyclocyprinae by Hoff 1942, p.100-101] Recent, Norway.

CYCLOLEBERIS [Cylindroleberis lobiancoi G. W. Müller 1894, p.220,pls.4,5] Recent Gulf of Naples.

CYLINDROLEBERINAE G. W. Müller $1906 \mathrm{~b}, \mathrm{p} \cdot 32$. n.subfam. of Cyprldinidae, for Cylindroleberis and Cyclasterope.

CYLINDROLEBERIS [Cypridina mariae Baird 1850,p.257,pl.17, f.5-7; Cypridina teres Norman 1861,p.280,pl.14, f.10] Brady 1868a,p.127 [Cypridinidae by Brady; Cypridinidae-Cylindroleberinae by Müller 1906b, p.32] Recent, North Atlantic.

CYLINDRUS [Cythere jurinei Münster 1850,p.62] Neviani 1928, p.72,106 [as "Gruppo" = subgenus or genus - of "Cythereis" by Neviani] Miocene to Recent, Italy by Ruggieri 1950,p.13. [ = Cytheretta by Ruggieri 1950a,p.9]. Not Cylindrus Fitzinger 1833,moll., by Neave 1939,p.929.

CYPRACEA Sylvester-Bradley 1949,p.130, a revision of the spelling of the superfamily Cypridacea Ulrich and Bassler. 
CYPRELLA [C. chrysalidea] Koninck 1841,p.19 [Cypridinidae by Bassler \& Kellett 1934,p.255] Carboniferous, Belgium.

CYPRETTA iC. tenuicauda] Vávra 1895,p.6 [as subgenus of Cypridopsis; Cypridae-Cyprinac-Cypridopsini by Bronstein 1947,p. 174] Recent, Zanzibar.

CYPRTA [C. punctata Jur., C. Joanna Baird, C. vidua Müll., C. semilunaris Fisch., C. ovum Jur. cited in order] Zenker 1854,p.77, [as subgenus of Cypris by Zenker; Cypridae-Candoninae by Müller 1912, p.129; Cypridae-Candoninae-Cyclocyprini by Bronstein 1947,p.182] Recent, Europe [Note: Brady and Norman 1889, p.68 designate Cypris (Cypria) exsculpta Fischer 1855,p.652,pl.19, f.36-38 as genotype, which they state "Cypria punctata striata Zenker $1854, \mathrm{p} \cdot 77, \mathrm{pl} .3 \mathrm{~A}$ " No such variety was named by Zenker on either p.77, not plate 3A, though his explanation of Taf.III. A. Cypris (Cypria) punctata Jurine. Fig.l. reads: "Schale der gestreiften Varietat."]

CYPRICERCUS [C. cuneatus] Sars 1895,p.37 [= Strandesia by Mïller 1912,p.186; but Cypridae-Cyprinae by Klie 1938a,p.113] Raised from South African mud. 
CYPRICONCHA [Cypris barbatus Forbes 1893,p.244,pl.37,f.2,

3; pl.38] Sars $1925, p \cdot 5$ [ = Candonocypris by

Furtos 1936b,p.503; but Cypridae-Cyprinae by

Dobbin 194l,p.183] Recent, Yellowstone National Park.

CYPRIDA Goldenberg $1870, \mathrm{p} .286$ [Genus attributed to Jones by Goldenberg, and used by him in the description of Cyprida elongata n.sp. from the Steinkohlenformation von Saarbrucken] = Candona by Bassler and Kellett 1934,p.236.

CYPRIDACEA (vel Ostracoda) Dana 1849,p.49. Used as a Tribe in place of Ostracoda, for the genera Cypris (Müller), 5 species; Cypridina (Milne-Edwards) 5 species; and Conchaecia new, 4 species.

CYPRIDACEA Ulrich and Bassler 1923,p.316-321 as a. Superfamily to include tha families: Beecherellidae, Bairdiidae, Cypridae, Cytherellidae, Entomidae, Cypridinidae, Entomoconchidae, Polycopidae, Darwinulidae, Barychilinidae.

CYPRIDAE Baird 1845,p.152, a family to include Cypris, Candona, and Cythere.

CYPRIDEA [no type designated by the author, who refers to the species described as Cypris by. Sowerby, Roemer, Dunker and Forbes in England and Germany . $\underline{\text { C. }}$ punctata Forbes designated erroneously by Anderson 
1939,p.293-296; Cypris granulosa Sowerby was designated properly by Sylvester-Bradley 1949, p.126, and by Swain 1949a,p.179] Bosquet 1852, p.47. [Cypridae-Rostocyprinae by Anderson 1939, p.292-293; Cypridae-Cyprideinae by Martin 1940, p.280-281; and by Sylvester-Bradley 1949,p.130] Jurassic, England.

CYPRIDEINAE Martin 1940,p.280-281, a subfamily of Cypridae to replace Rostocyprinae Anderson, which had been improperly named, for the genus Cypridea.

CYPRIDEIS [Candona torosa Jones 1850a,p.27,pl.3,fig.3a-e] Jones 1857, p.20 [Cytheridae-Cytherideinae by Sars 1925,p.154] Recent, England.

CYPRIDELLA [C. cmuciata] Koninck 1841,p.20 [Cypridinidae by Bassler and Kellett 1934,p.256] Carhoniferous, Belgium.

CYPRIDELLA [C. lemurensis] Vávra 1895,p.7 [Cypridae by Vávra] Recent, Zanzibar. [a homonym of Cypridella Koninck. $[=$ Cypretta by G. W. Müller 1912,p.206]

CYPRIDELLINA [ 8 species from the Mountain Limestone, none named; Jones 1873a, p.74; also Jones 1873b,p.410, where the species are itemized, but all placed under the genus Cypridinella! 
CYPRIDELIINA $[$ C. clausa, C. burrovii, C. burrovii var longnoriensis, C. intermedia, C. elongata, C. elongata var. hibernica, C. Ealea, C. vomer, C. alata, C. bosqueti n.spp] Jones and Kirkby, Genus novum 1874,p.25-31 [Cypridinadae by JEK; Cypridinidae by Bassler and Kellett 1934,p.44,258, who designated $\underline{C}$. clausa as genotype.] Carboniferous, Ireland.

CYPRIDIDAE Baird 1850,p.viii, fide Jones 1857,p.6 [tot seen, my film copy lacks the Introduction, but Jones indicated that Baird had corrected the spelling Cypridae used later, p.139 and following, in the body of his work.]

CYPRIDIDAE Kaufmann 1900a,p.104, a family of Podocopa to include the subfamilies: Notodromadinae, Herpetocypridinae, Cypridinae, Cypridopsinae, Cyclocypridinae, Illyocypridinae, Candoninae, and Pontocypridinae.

CYPRIDIFORMIA Daday 190C,p.32,124,183,304, a Tribe of subfam. Cyprinae [subtribe,p.183] for the genera: Cypridella, Cypretta, Cypridopsis, Potamocypris, Pionocypris.

CYPRIDINA [C. renaudi] Milne-Edwards 1840,p.409 [Cypridinadae by Baird 1850a] Recent, Indian Ocean. 
CYPRIDINADAE Baird 1850a,p.176, a family for the genus

Cypridina; also Sars 1866,p.99-102; and Jones and Kirkby 1874. = Cypridinidae under Article 4 of the International Rules of Zoological Nomenclature.

CYPRIDINAE Kaufman 1900a,p.104, a subfamily of Cyprididae, to include Cipris, Strandesia, Centrocypris, Acocypris, Stenocypris, Cyprinotus, Fieterocypris, Zonocypris, Chlamydotheca, Eurycypris, Pachycypris, Cypretta, Cypridella, Pionocypris, Acanthocypris.

CYPRIDINFLLA [7 species: 3 names: C. cummingi, C. monitor, and C. vomer] Jones $1873 \mathrm{a}, \mathrm{p} .74$; also Jones $1873 \mathrm{~b}$, p.410 gives a brief description of the genus, names all 7 species: $\underline{C}$ cummingil, $\underline{C}$. superciliosa, $\underline{\text { C. clausa, }} \underline{\text { C. bosquet }}$, $\underline{\text { C. maccoyana, }}$. . vomer, $\underline{\text { C. }}$ monitor. He then says: "The last is typical of the peculiarly produced carapace, with antero-ventral prow beneath the retiring sinus, a form which reminds us of the profile of such iron-clad naval 'rams' as the "Monitor' and the 'Merrimac'". The genotype is thercfore Cypridinella monitor Jones 1873, not $\underline{C}$. cummingii as designated by Bassler and Kellett $1934, \mathrm{p} \cdot 44$. It should be noted that this genus was described in more detail, together with the above mentioned species, all indicated as new, by Jones and Kirkby 1874,p.21-25. [Cypridinidae by Bassler and Kellett 1934,p.265] Carboniferous, Belgium. 
CYPRIDINIDAE, as used by Ulrich $1894, \mathrm{p} .632$, included:

Cypridina, Cypridinella, Cypridellina, Sulcuna, Cypridella, Cyprella, Bradycinetus, Philomedes, Rhombina, and Cyprosis. By G. W. Müller 1906, p.12 33, it was divided into the following subfamilies: Cypridininae, Sarsiellidae, Cylindroleberinae.

CYPRIDINIFORMES Skogsberg 1920,p.158,159-544, a suborder to include the families Cypridinidae, with subfamilies Cypridininae and Philomedinae; Rutidermatidae, Sarsiellidae; and Asteropidae.

CYPRIDININAE Dana 1853,p.1281, subfamily of Halocypridae, for the genus Cypridina.

CYPRIDINODES [C. favus] Brady 1902,p.187 [Cypridinidae by Brady] Recent, "Locality appears to have been lost." [ = Cypridina by G. W. Müller 1912,p.13]

CYPRIDINOPSIS Armstrong 1871,p.26 [used by Armstrong in a checklist for two species: C. youngiana $\left(J_{\bullet} G_{0}\right)=$ Cythere? youngiana Jones and Kirkby 1867, p.223; and $C_{\text {. simplex }}$ J. \& $K_{0}=$ Polycope simplex (Armstrong) by Jones $1873 \mathrm{~b}, \mathrm{p} \cdot 412$. Both species are referred to Polycope by Bassler and Kellett 1934,p.434, 435 .

CYPRIDONOTUS Claus 1893,p.197, a mistake in spelling of Cyprinotus Brady 1886 a. 
CYPRIDOPSELLA [Monoculus villosus Jurine 1820,p.178,pl.19, f.14,15, designated by Sars 1925,p.143 = Candonella villosa Vávra 1898a,p.13] Kaufmann 1900c,p.131, new name for Candonella of Vávra [Cypridae-Cyprinae-Cypridopsides by Sars 1928,p.x] Recent, Europe.

CYPRIDOPSIDES Sars 1925,p.134,a group of Cypridae-Cyprinae for the genera Pionocypris, Cypridopsis, and Cypridopsella.

CYPRIDOPSINAE Kaufmann 1900a,p.106, a subfamily of Cypridae to include: Cypridopsis, Paracypridopsis, Cypridopsella, Candonella, Zonocypris, Oncocypris, Potamocypris.

CYPRIDOPSINI Bronstein 1947,p.154, a Tribe of Cypridae-Cyprinae to include: Cypridopsis, Potamocypris, Cypretta.

CYPRIDOPSIS [C. vidua (O. F. Müller), aculeata (Iilljeborg), villosa (Jurine); Brady and Norman 1889,p.89 and Sars 1889, p.53 each designated $\underline{C}$. vidua as genotype. Brady and Norman 1896,p.725 overlooked their previous designation and made $\underline{C}$. vidua the genotype of Pionocypris; and Sars 1924,p.155 accepted Pionocypris, and overlooking his own designation of 1889, [Sars 1924,p.157-158] designated $\underline{C}$. aculeata as genotype. The 1889 designa-

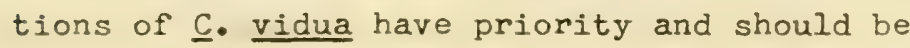


recognized, as indeed they are by most workers] Brady 1868a,p.117 [Cypridae by Brady; Cypridae-Cyprinae-Cypridiformia by Daday 1950,p.251] Recent, North Europe - and North America.

CYPRIFORMES Skogsberg 1947,p.158, a Tribe of the subfamily Cyprinae for Cyprinotus and Heterocypris.

CYPRIIIA [C. arcuata] Sars 1924a, p.169 [Cyprinae near Potamocypris by Sars] Recent, Africa, raised from dried mud.

CYPRINAE G. W. Müller 1894,p.241, a subfamily of Cypridae for the genera Macrocypris and Aglaia.

CYPRINAE G. W. Müller 1900,p.45, a subfamily of Cypridae for the genera Cypris, Notodromas, Cypridopsis, Stenocypris, Eurycypris, Cyprinotus, Potamocypris, Cyprois.

CYPRINAE Daday $1900, p \cdot 122,303$, a subfamily of Cypridae with Tribes Ctenocyprina and Cypridiformia, and subtribes Zygopsida and Synopsida.

CYPRINAE Daday 1905 p.235, a subfamily of Cypridae to include Tribe Ctenocyprina, with genera Eucypris and Chlamydotheca, and Tribe Cypridiformia, with the genus Cypridopsis. . 
CYPRINAE Sars 1923,p.68, a subfamily of Cypridae is divided into these groups: Paracyprides, Candonides, Cyclocyprides, Notodromides, Ilyocyprides, Eucyprides, and Cypridopsides.

CYPRINOTINI Bronstein 1947,p.135, a Tribe of the subfamily Cyprinae for Cyprinotus and Heterocypris.

CYPRINOTOIDES [Cyprinotus (Cyprinotoides) somalicus] Nasi 1928,p.51 [a subgenus of Cyprinotus by Masi] Recent, Somaliland.

CYPRINOTUS [C. cingalensis] Brady 1886a,p.301 [CypridaeCyprinae by Sars 1923,p.277] Recent, Ceylon.

CYPRIONE [C. bristovii] Jones 1885e,p.343 [Darwinulidae by Martin 1940,p.318] Jurassic, England.

CYPRIS [C. pubera designated by Baird 1846,P.413] O. F. Müller 1776,p.198-199; 1785,p.48 [Cyprididae-Cypridinae by Kaufmann 1900a,p.105] Recent, Europe [Over 500 specific names have been used with this genus, but most have been transferred elsewhere].

CYPROIDEA Dana 1853,p.1277, a "Tribe", used in same sense as we now use Ostracoda, and divided into family Cypridae, with subfamilies Cyprinae and Cytherinae; and family Halocypridae, divided into subfamilies Cypridininae and Halocyprinae. 
CYPROIS [C. monacha Miill., C. dispar Fisch.] Zenker 1854, p.80. [Cypridae-Notodrominae by Hoff 1942,p.147] Recent, Europe. Note: Lilljeborg 1853, had monotypically used $\underline{C}$. monacha O. F. Müller as the

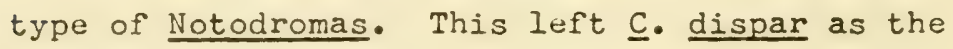
genotype of Cyprois.

CYPROSINA [C. whidbornei] Jones 1881c,p.338 [Cyprosinidae by Whidborne 1890,p.52] Devonian, England.

CYPROSINIDAE Whidborne $1890, p .52$, a family for the genus Cyprosina Jones.

CYPROSIS [C. haswellii] Jones 1881c,p.338 [a Cypridinad by Jones; Cypridinidae by Bassler E Kellett 1934 , p.268] Silurian, Scotland.

CYRTOCYPRIS [C. subovata] CoryelI and Williamson 1936,p.7 [Bairdiidae by CEW.] Silurian, Indiana.

CYRTOCYPRUS COryell and Williamson 1936,p.7, a miss-print for Cyrtocypris.

CYTHERALISON [C.fava] Hornibrook 1952,p.66 [Incertae sedis by Hornibrook] Recent, New Zealand, but with range from Eocene.

CYTHERACEA Ulrich and Bassler 1923a,p.322, a Superfamily where they place only the family Cytheridae Zenker, sic. 
CYTHERIDAE Baird 1850a,p.162 [ = Cytherinen Roemer 1838]

a family to include Cythere, O. F. Müller, and Cythereis, Jones.

CYTHERINAE Dana 1853,p.1281 [ = Cytheridae Baird 1850] a subfamily of Cypridae, to include Cythere and Cythereis.

CYTHERE [c. viridis, C. Iutea, C. flavida, C. gibba, C. gibbera] O. F. Mülller 1785, p.63 [‥ lutea designated genotype by Brady and Norman 1889, p.125; the carapace first described in detail by Blake 1931a,p.160-161] Recent, North Atlantic.

CYTHEREIS [ 9 species; the 6th: C. ciliata (Reuss) = Cytherina ciliata Reuss 1846,p.104,pl.24,f.17= Cytherina ornatissima Reuss $1846, p \cdot 104, p 1.24, f \cdot 12$, 13 = Cythereis ornatissima (Reuss) designated by Sutton and Williams 1939,p.562; and by Triebel 1940,p.174-180 who described carapace in detail, and by Sylvester-Bradley 1.948d,p.792-797] Jones 1849,p.14-15 [as subgenus of Cythere by Jones; Cytheridae-Cytherinae by Triebel 1940,p.174] Cretaceous, Bohemia. 
CYTHLREIS, Skogsbere 192k,p.38-56, descrihed as a suhgenus of Cythereis Jones, a Cretaceous genus, but actually based for the most part on Cythereis (Cythereis) montereyensis, n.sp., living California. Skogsberg indicates the type must be one of Jones species, but does not indicate which. He divides his own species into 6 groups, some of which are quite different from $\underline{c}$. (‥) montereyensis and would fall withir Hemicythere Sars 1925. This subgenus $=$ Eucythereis Klie 1940, by Klie in his description.

CYTHERELLA [6 species, the 1st. Cytherina ovata Roemer 1840, p.104,pl.16,f.2l being designated genotype by Ulrich 1894,p.684] Jones 1849,p.28 [as subgenus of Cythere by Jones; Cypridae-Cytherinae by Brady 1866,p.361; Platycopa-Cytherellidae by Sars 1866, p.125-127] Crateceous, Germany.

CYTHERELLIDAE Sars 1866,p.124, a family of Podocopa for the genus Cytherella.

CYTHERELLIFORN ES Skogsberg 1920,p.158, a suborder "for all Cytherellids." = Podocopa Sars 1866.

CYTHERELLINA [Beyrichia siliqua Jones 1855,p.90,pl.5,f.22] Jones and Holl 1869,p.215 [ = Bythocypris by Bassler and Kellett 1934,p.282, but not by others; Cytherellidae by Bouček 1937,p.1] Silurian, England. 
CYTHERELIOIDEA [Cythere (Cytherella) williamsoniana Jones $1849, \mathrm{p} \cdot 31, \mathrm{pl} \cdot 7, \mathrm{f} \cdot 26 \mathrm{a}-1]$ Alexander 1929,p.55 [Cytherellidae by Alexander] Cretaceous, England.

CYTHERETTA [C. rubra G. W. Müller 1894 = Cytherina subradiosa Roemer $1838, \mathrm{p} \cdot 517, \mathrm{pl} \cdot 6, \mathrm{f} \cdot 20$ = Cytheretta subradiosa (Roemer) by Ruggieri 1950a,p.9] G. W. Nïller 1894, p.382 [Cytheridae-Cytherettinae by Triebel 1952, p.16] Pliocene to Recent, Italy.

CYTHERETTINAE Triebel 1952,p.10, a subfamily of Cytheridae for Cytheretta and Paracytheretta.

CYTHERIDEA [ 4 species, the lst, Cythere mülleri Münster 1850, p.62, was designated genotype by Brady and Norman 1889,p.172] Bosquet 1852,p.37 [Cytheridae-Cytherideinae by Sars 1925,p.152] Tertiary, Oligocene for the most part, Osnabrück, Cassel, Paris, Bordeaux. The type species was first figured by Roemer 1836,pl.6,fig.6. The genus has received much modification in recent years, see particularly Stephenson 1936, and Goerlich 1953.

CYTHERIDEINAE Sars 1925,p.152, a subfamily of Cytheridae to include Cytherissa, Cyprideis, Cytheridea, Eucythere, and Krithe.

CYTHERIDEIDAE Sylvester-Bradley and Harding 1953,p.755, a family for Cytheridea, Fericytherideis, Neocytherideis. 
CYTHERIDEIDINAE Sylvester-Bradley and Harding 1952,p.754755, a correction of Puri's Cytherideisinae $=$ Cyprideinae.

CYTHERIDEIS Jones 1856a,p.157,158 [2 species: C. unisulcata, C. unicornis] Jones $1857, \mathrm{p} .46$ (10 species) as a subgenus of Cythere. The genus is dead. Sylvester-Bradley and Harding 1953 designated C. unicornis as genotype, which was the first time one of the original 2 species was designated. $\underline{C}$.

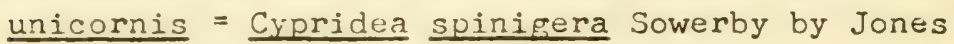
and Sherhorn 1887,p.386, and therefore Cytherideis bccomes a synonym of Cypridea Bosquet 1852. Many of the more than 100 species which have been described under the name Cytherideis should be transferred to Cushmanidea Blake 1933, or Pontosythere Dubowsiky 1939.

CYTHERIDEISINAE PUri 1352,p.905, for Cytherideis, Copytus, Pontocythere, Krithe, Cushmanidea, Sahnia, and Neocytherideis. $[=$ Cytherideidinae Puri, by Sylvester-Bradley and Harding 1953,p.754-755, a corrrction of the ending of Puri's name; = Cyprideinae Martin 1940, by Sylvester-Bradley and llarding.

CYTHERIDELIA [C. ilsovayi] Daday 1905,p.261 [Cytheridae by Daday ] Recent, Paraguay. 
CYTHERINA [C. viridis, C. lutea] Lamarck 1818,p.125

[ = Cythere 0. F. Múller by Bosquet 1847, p.356]

CYTHERINAE Dana 1853,p.1281, subfam. of Cypridae for Cythere and Cythereis.

CYTHERISSA [Cythere lacustris Sars 1863,p.222 (p.30 of separate)] Sars 1925,p.152 [Cytheridae-Cytherideinae by Sars] Recent, fresh-water, Europe. For additional discussion and species seen Bronstein 1939, 1947.

CYTHERITLS [C. insignipes] Sars 1926,p.10 [Cytheridae by Sars; = a subgenus of Entocythere by Hoff and Cytheridae-Entocytherinae by Hoff 1942,p.69] Recent, commensal on crayfish, Canada.

CYTHEROIS [C. virens n.sp. = Paradoxostoma fischeri Sars 1866,p.96] G. W. Muller 1884,p.15-17 [CytheridaeParadoxostominae by G. W. Müller 1894,p.308] Recent, Europe.

CYTHEROMA [C. variabilis] G. W. Milller 1894,p.349 [Cytheridae-Cytherominae by E.lofson 1941,p.339] Recent, Gu.1f of Naples.

CYTHEROMINAL LIOf son 1939,p.15, a subfamily of Cytheridae for the genus Cytheroma.

CYTHEROMORPHA [C. albula n.sp., and C. claviformis n.sp.]

Hirschmann 1909,p.290-292 [C. albula = Cythere fuscata Brady 1869a,p.4 5, 47,pl.7.f.5-8, by 
Hirschmann 1912,p.44, and $\underline{\text { C. fuscata }}$ was designated genotype by Sars 1925,p.177; Cytheridae-Cytherinae by Elofson 1941,p.276] Recent, N. W. Europe.

CYTHEROPSIS M'COY $1849, \mathrm{p} \cdot 414$, no species mentioned;

Cytheropsis aldensis M'Coy 1851,p.387 is first species described. $[?=$ Pontocypris by Bassler and Kellett $1934, p .436$, but type still requires re-study] Cambro-Silurian limestone of Aldens, Ayrshire by M:Coy •

CYTHEROPSIS [C. argus] Sars 1866,p.57-58, Recent, Norway• Junior homonym of Cytheropsis M'Coy, = Eucythere Brady 1868,p.429.

CYTHEROPTERON [given by Sars as "Cytheropteron convexum (Baird)" on mistaken identity = Cythere latissima Norman $1865, \mathrm{p} .19, \mathrm{pl} \cdot 6, f \cdot 5-8$, by Brady $1868 \mathrm{a}$, p.448; by Brady \& Norman 1889,p.207; and by Sars 1926,p.223] Sars 1866,p.79,80 [Cytheridae-Cytherurinae by G. W. Muiller 1894,p.286] Recent, North Atlantic. Perhaps 300 species, Recent and fossil.

CYTHERURA [Cythere gibba (male) and Cythere gibbera (female) O. F. Müller 1785 - designated by Brady \& Norman 1889,p.190] Sars 1866,p.69,70. [Cytheridae-Cytherurinae by G. W. Múller 1894,p.286] Recent, North Atlantic. 
CYTHERURINAE G. W. Muller $1894, \mathrm{p} .286$, a subfamily of Cytheridae for the genera Cytherura, Cytheropteron and Eucytherura.

DALEIELLA [Cythere corbuloides Jones and Holl 1869,p.211, pl.15] Boućek 1937,p.7 [Bairdiidae by Boucek; Incertae Sedis by Agnew 1942,p.758; Bairdiidae by Morris and Hill 1952,p.13] Silurian, England.

DAPHNIA M'Coy 1844,p.164, non Müller 1776, used in the description of Daphnia primaeva n.sp. = Cypridina primaeva (McCoy) by Bassler and Kellett 1934,p.263.

DARWINELIA Brady and Robertson $1872, \mathrm{p} \cdot 50$, nom.nov. for Polycheles BER, preoccupied. Not Darwinella Müller 1865 (Spong.) = Darwinula Brady and Robertson 1885, nom.nov.

DARWINELIIDAE Brady, Crosskey, and Robertson $1874, \mathrm{p} \cdot 110,112=$ Darwinulidae Brady and Norman 1889,p.121.

DARWINULA [Polycheles stephensoni Brady and Robertson 1870, p.25,pl.7,f.1-7; pl.10,f.4-14] Brady and Robertson in Jones $1885 \mathrm{e}, \mathrm{p} .346$ [Darwinulidae by Brady and Noman 1889,p.121] Recent, Europe.

DARWINULIDAE Brady and Norman 1889,p.121 for the genus Darwinula. 
DELOIA [D. serrata) Croneis and Thurman 1938,p.307

[Kloedenellidae by CET; Kloedenellidae-Beyrichiopsiinac by Henningsmoen 1953,p.272.

DENISONELLA [Denisonia cincta Croneis and Bristol 1939, p.76,pl.4,f.13] Croneis and Bristol,p.777, new name for Denisonia Croneis and Bristol 1939,p.76, (not Krefft). [Kloedenellidae by CEB; KIoedenellidae-Beyrichiopsiinae by Henningsmoen 1953, p.272] Mississippian of Illinois.

DENISONIA [D. cincta] Croneis and Bristol 1939,p.76 (non Krefft, 1869) = Denisonella Croneis and Bristol 1942,p.777, new name.

DEPRANELLA [D. crassinoda, orig. designation] Ulrich 1890, p.117,118 [Note this description was accompanied by description also of Depranella nitida, n.sp., Depranella macer, n.sp., Depranella ampla,n.sp•, and Depranella elongata, n.sp. - all using the same spelling of Depranclla. Ulrich, 1894,p.670, however changed the spelling to Drepanella, and subsequent workers have followed the latter spelling. It would appear that Ulrich in 1890 had made no obvious typographical error, or lapsus calami, and that under the Rules he was not permitted to change the name. For a discussion of 
the Rules governing this case, sec Moore, Weller, and Knight, 1942, and particularly their discussion of "Graphiadactyllis versus Graphiodactylus," p.256,257]. [Zygobolbidae-Drcpanelinae $($ sic $)=$ Depranellinae, by UIrich and Bassler 1923,p.293] Ordovician, Kentucky.

DEPRANELIIDAE - a change in the spelling of Drenanellidae, Swartz $1936, p \cdot 552$, to correspond with the original spelling of its type genus Depranella.

DEPRANELIINAE - a change in the spelling of Drepanellinae, Ulrich and Bassler 1923,p.308, to correspond with the original spelling of its type genus Depranella. DIAGONELLA Swartz 1945a,p.1205, ["DrepanelIidae" by Swartz] no genotype given.

DIBOLBINA [D. cristata] Ulrich and Bassler 1923a,p.312 [Beyrichiidae by Bassler and Kellett 1934,p.285; Beyrichiidae-Beyrichiinae by Henningsmoen 1953, p.227,238,270. Silurian, Maryland.

DICRANELLA [D. bicornis] Ulrich 1894,p.664 [Beyrichiidae by Ulrich; Primitidae-Primitiinae by Bassler and Kellett 1934,p.19,286; Primitiidae-Bolliinae by Kay 1940a,p.260; Hollinidae-Ctenonotellinae by Schmidt 1941,p.48; Tetradellidae-?Piretellinae by Henningsmoen 1953,p.219,267] Ordovician,Minnesota. 
DIHOCMOCHILINA [Isochilina grandis var. Iatimarginata Jones $1891, p .78, p 1.10, f .1-4]$ Teichert 1937a, p.153;

[Leperditiidae-Isochilininae by Swartz 1949,P.325] Silurian, Canada.

DIHOGMOCHILUS Neave, 1950, vol.5,p.74, error for Dihopmochilina Teichert 1937a.

DILOBELLA [D. typa] Ulrich 1894, p.672, [Beyrichildae by Ulrich; Primitiidae-Primitiinae by Bassler and Kellett 1934,p.20; Primitidae-Dilobellinae by Kay 1940a,p.2; Tetradellidae by Swartz 1936, p.551; ?hollinidae-Tetradellinae by Schmidt 1941, P.37; Tetradellidae-Tetradellinae by Henningsmoen 1953,p.266; Ordovician of Minnesota.

DILOBELLINAE Kay $134_{4}$ Oa,p.256, a subfamily of Primitildae to include Dilobella and Ceratonsis. Henningsmoen $1953,0.197,266$ has included this subfamily in the Tetradelinae of Swart.

DIJCOIDELLA [D. simplex] Croneis and Gale 1939,p.276 [Kirkbyidae by CErG; Beyrichiacea of uncertain family by Henningsmoen 1953,p.273) Mississippian of Illinois.

DITHYROCARIS Scouler, considered by Jones $1850 \mathrm{~b}, \mathrm{p} .64$ to belong to the Phyllopoda, and who reported Dithyrocaris permiana, and D. glypta n.sp., from 
the Carboniferous or Permian of England. Both species have been referred to the genus Kirkbya.

DIZYGOPLEURA [D. swartzi] Ulrich and Bassler 1923a,p.313 [Kloedeneliidae by Bassler \& Kellett 1934,p.30; Kloedenellidae-Kloedenellinae by Henningsmoen 1953,p.270] Silurian, Maryland.

DIZYGOPLEURINAE Egorov 1950,p.?, by Polenova 1952,p.104, who places here Dizygopleura.

DOLEROCYPRIA [D. taalensis] Tressler 1937,p.200,201

[Cypridae by Tressler] Recent, Philippines.

DOLEROCYPRIS [Cypris fasciata O. F. Müller 1785,p.53,pl.4, f.1-3] Kaufmann $1900 b, p \cdot 277,278, p l .20, f .13-15$; pl.22,f.1-3 [Cypridac-Cyprinae by G. W. Müller 1912,p.191] Recent, Europe.

DOLOCYTHERIDEA [Cytherina hilseana Roemer 1841,p.104,pl.16, f.17] Triebel 1938a,p.494 [As subgenus of $\mathrm{Cy}-$ theridea by Triebel] Cretaceous, Germany.

DOLORIA [Cypridina (Doloria) levis] Skogsberg 1920,p.223225 [as a subgenus of Cypridina. Cypridinidae-Cypridininae by Skogsberg] Recent, South Georgia. DONALDSONCYTHERE [Entocythere donaldsonensis Klie 1931,p.334, f.1-9] Rioja 1942,p.686 [As Subgenus of Entocythere; Cytheridae-Entocytherinae] Recent, Kentucky• 
DONELIINA Egerov [1950?],p.?, discussed by Polenova 1952, p.106 under Kloedenellidac-Cavellinae.

DREPANELLIDAE Swartz 1936,p.552, a family to include the following genera: Drepanella,$[\underline{\text { sic. }}$. Depranella,$]$ Scofieldia, Jonesclla, Bollia, and ?Ulrichia, ?Polyzygia. $[=$ Depranellidae $]$.

DREPAIELLINAE Ulrich and Bassler 1923,p.308, as a subfamily of Zygobolbidae, for the genera: Drepanella [sic. = Depranella], Drepanellina, Scofieldia, Mesomphalus. [ = Depranellinae $]$.

DREPANELIA U1rich $1894, \mathrm{p} .670$ iUlrich indicates = Depranella, an error; but under the Rules this change is not permissable, as the error is not an obvious error of transcription; 5 species having been described with the same spelling, see Ulrich 1890,p.117, 118. For full discussion of the Rules governing this case see Moore, Weller and Knight 1942). $[=$ Depranella Ulrich 1890].

DREPANELIINA [D. clarki] Ulrich and Bassler 1923,p.308 [Zygobolbidae-Drepanellinae by UEB; Beyrichiidae-Beyrichiinae by Henningsmoen 1953,p.232,236,238, 269] Silurian, Maryland, Pennsylvania.

DRIESCHIA [D. mammillata] Brehm 1923,p.339 [near Centrocypris and Stenocypris by Brehm] Recent, China. 
DUHMBERGIA [Ctenobolbina duhmbergi Opik 1937,p.96,pl.4,f.10, $11, p 1.6, f .5]$ Schmidt 1941,p.39 [as Subgenus of Ctenobolbina; Hollinidae-Tetradellinae by Schmidt] Ordovician, Esthonia. [ = Piretella, Ctenobolbina duhmbergi Ópik, being a young instar of Piretella margaritata Öpik, by Henningsmoen 1953,p.218,219.]

E

ECTODEMITES [E. primus] Cooper 1941,p.49 [Kirkbyidae-Amphissitinae by Cooper] Mississippian of Illinois [as a subgenus of Amphissites by Polenova 1952,p.117]

ECTOPRIMITIA [Primitia corrugata Krause 1892,p.386,pl.21, f.12] Bouček 1936,p.45 [As subgenus of Haploprimitia, Primitiidae-Primitinae by Boucek; Eurychilinidae-?Euprimitinae by Henningsmoen 1953,p.251,272] Ordovician drift, Germany•

EDITIA [E. elegantis] Brayer 1952,p.170, [Kirkbyidae by Brayer] Mississippian of Missouri.

ELLESMERIA [E. ovata] Tolmachoff 1926,p.35 [Barychilinidae by Bassler \& Kellett 1934,p.47,296] Devonian, Ellesmereland, Arctic America.

ELIIPSELIA [E. obliqua] Coryell and Rogatz 1932,p.390 [Cytheridae by CER; Kloedenellidae by Bassler and 
Kellett 1934,p.297; Kloedenellidae-Kloedenellinae by Henningsmoen 1953,p.242,270] Permian of Texas.

ELPE [E. inchoata] Barrande 1872,p.510 [Entomidae by Bassler and Kellett 1934,p.297; preoccupied by Elpe Robineau-Desvoidy 1863 = Elpezoe Pribyl, 1950, p.121, new name] Devonian, Bohemia, Germany.

ELPEZOE Přribyl 1950,p.121, new name for Elpe Barrande. [Entomoconchidae by Pribyl] Devonian, Bohemia.

ELPIDIUM [E. bromeliarum] Fritz Mưller 1880,p.386-388, f.1-15; also 1881,p.27-34,pl.2 [Cytheridae by Klie 1939-1940, part VIII,p.301] Recent, in leaves of Bromelia, Brazil. [ = Metacypris by Tressler 1941, p.264]

ELPINELLA [Leperditia radiata Ulrich $1879, \mathrm{p} \cdot 9, \mathrm{p} 1.7, \mathrm{f} .2 \mathrm{a}$, b] Pribyl, in Pribyl and Snajdr 1950,p.128,139, 171 [Entomoconchidae?] Ordovician, Ohio.

ENDOLOPHIA [E. chariessa] Kesling 1954,p.174 [Barychilinidae by Kesling] Devonian, Ohio.

ENTOCYTHERE [E. cambaria] Marshal1 1903,p.117-136,pls.10-12 [Cytheridae-Entocytherinae by Hoff 1942b,p.65] Recent, Wisconsin, on gills of cray-fish. 
ENTOCYTHERINAE Hoff $1942 b, p .65$, a subfamily of Cytheridae for Entocythere, with Cytherites, as a subgenus, and Sphaeromicola.

ENTOMIDELLA [Entomis divisa Jones 1861,p.137 and Leperditia buprestis Salter $1865, \mathrm{p} .285]$ Jones $1873 \mathrm{c}, \mathrm{p} .416$ [Entomidae by Jones] Jones $1884 a, p .401$ designated E. buprestis (Salter) as genotype. Ulrich and Bassler 1931,p.98,99 considered this species under the Conchostraca, as a species of uncertain affinities. E. buprestis was described from the Cambrian of Wales. [ = a Cambrian branchiopod by Bassler and Kellett 1934,p.381].

ENTOMIDACEA Schmidt 1941,p.72, a superfamily to include the families Entomidae and Bolbozoidae. [ ENTOMOZOACEA Pribyl 1951,p.103].

ENTOMIDAE Jones $1873 a, p .78$, a family separated from the Cypridinadae for the genera Entomis and Entomidella. $[=$ ENTOMOZOIDAE Pribyl 1951,p.104].

ENTOMIS [E. tuberosa] Jones 1861,p.137 [Entomidae by Jones 1873a,p.78] Silurian, Scotland. [Preoccupied by Entomis Herrich-Schaeffer 1856; = Entomozoe Pribyl 1950,p.107, new name]. 
ENTOMOCONCHACEA Sylvester-Bradley 1953,p.127-134,pls.7,8, a superfamily belonging to the Myodocopa, to include the family Entomoconchidae, with the genus Entomoconchus, and the family Cyprosinidae, with the genus Cyprosina. = Entomozoacea Pribyl, 1951.

ENTOMOCONCHIDAE Brady $1868 \mathrm{~b}, \mathrm{p} \cdot 358,359$, a family of Myodocopa to include Entomoconchus and Heterodesmus.

ENTOMOCHONCHUS [E. scouleri] MPCOy 1839,p.91 [Entomoconchidae by Brady 1868b,p.358] Carboniferous, Ireland.

ENTOMOZOACEA Pribyl 1051,p.103, a superfamily for the families Entomozoidae and Bolbozoidae.

ENTONOZOE [Entomis tuberosa Jonea 1861,p.137,pl.2,f.5] Pribyl 1951,p.105 [new name for Entomis Jones 1861, not Herrich-Schaeffer 1856]; Entomozoacea-Entomozoidae-Entomozoinae by Pribyl] Silurian, England.

ENTOMOZOIDAE Pribyl 1951,p.104, nom•nov., for Entomidae Jones 1873; divided into subfanilies Entomozoinae and Bouciinae.

ENTONOZOINAE Pribyl 1951,p.105, a subfamily of Entomozoidae for the genera and subgenera: Entomozoe (Entomozoe), E. (Richteria), E. (Nehdentomis), Richterina (Richterina), R. (Eossirichterina), Franklinella, Rhomboentomozoe, and Pseudoentomozoe. 
ENTOPRIMITIA [Primitia hattingensis Matern 1929,p.24,pl.1, f.1la,b] Kummerow 1939,p.19 [Primitiidae-Primitiinae by Kummerow; Aechminidae by Agnew 1944 , p.218,219; Family uncertain by Henningsmoen 1953, p.273] Devonian, Germany. [Note: Kummerow 1953, p.62 apparently forgot all about having described Entoprimitia, when he described Omphalentomis, n.gen., as he indicated Primitia hattingensis belonged to Omphalentomis, differing from the designated genotype only in being longer made no reference whatever to Entoprimitial

EOCONCHOECIA [E. mucronata] Moberg 1895,p.9 [Halocypridae? by Moberg; Beyrichildae by Bassler and Kellett 1934,p.309; Acronotellidae by Swartz 1936,p.549; Hollinidae-?Ctenentominae by Schmidt 1941,p.34; Beyrichiacea of uncertain family by Henningsmoen 1953,p.273] Silurian, Sweden.

EOCYTHERELLA Bonnema 1933b,p.155, a suggested genus to hold Cytherella smithi Jones 1887,p.192,pl.7,f.15,16; Cytherella troedssoni, n.name Bonnema 1933b,p.155 for Primitia tenera Troedsson 1918,p.47,pl.2, f.3; and perhaps Cytherella quaesita Roth 1929, p.307, pl.38,f.27a-c. The first two from the Silurian of Gotland. 
EOCYTHEROPTERON [Cytheropteron bilobatum Alexander 1929, p.104,pl.10,f.14,15] Alexander 1933,p.195,196. [Subgenus of Cytheropteron by Alexander] Lower Cretaceous, Texas.

EOLEPERDITIA [Cytherina fabulites Conrad 1843,p.332]

Swartz 1949,p.317 [Leperditiidae-Leperditiinae by Swartz] Ordovician, Wisconsin.

EREMOS [E. bryograptorum] Westergaard,mscr. Moberg and Segerberg 1906,p.75,pl.3,f.24 [Compared to Isochilina and Bradoria by authors; Conchostraca by Ulrich and Bassler 1931,p.61] Ordovician, Sweden.

ERIDOCHONCHA [E. mugosa] Ulrich and Bassler 1923,p.297 [Leperditellidae by Bassler and Kellett 1934, p.310; Aparchitidae by Kay 1940a,p.240,247;

Primitiidae by Levinson 1951,p.556; ?Leperditellidae-?Eridoconchinae by Henningsmoen 1953,p.251,256, 275.] Ordovician, Ohio.

?ERIDOCONCHINAE Henningsmoen 1953,p.255,275, a subfamily of ?eperditellidae, for the genera: Eridoconcha, Cryptophyllus, Milleratia, Schmidtella, ?Paraschmidtella.

ERIELIA [E. robusta] Stewart and Hendrix 1945a,p.95 [Quasillitidae by SEH] Devonian, Ohio. 
ERPETOCYPRIS 「Candona reptans Baird 1850,p.160,pl.19,f.3, 3a) Brady and Norman 1889,p.84 Recent, England. Brady and Norman overlooked Baird's 1846,p.414, designation of Candona reptans, as genotype of Candona, instead they designated $\underline{C}$. candida genotype of Candona, and $\underline{C}$. reptans as genotype of Erpetocypris. Baird's designation of a genotype was probably the first such designation in the field of Ostracods, and under the Rules should hold. Erpetocypris Brady and Noman 1889 and Herpetocypris Brady and Norman 1896, a chanpe in spelling, are therefore synonyms of Candona, and the large Candona candida group of species needs a new name; apparent.ly Typhionypris.

DRYTHROCYPRIS (Cythere (Rairdia) mytiloides Norman 1862, p.50,pl.3,f.1-3, designated by Sars 1923,0.50-51] G. W. Müller 1894, p.256. Recent, Europe. [E. serrata, one of 6 species described by G. W. Müller 1894 was considered by both Sars and Muller to = Pontocypris sermulata (Sars) 1866, which in turn = Cythere mytilnides Norman 1862 - a species which Brady and Norman had in $1889, \ldots .107$ desienated as genotype of Pontocynris. Erythrocynris therefore * Pontocypris. See Sylvester-Bradley TMarch 1946 * Jan $1947, \mathrm{p} .193]$. 
ESCASONA (E. rutellum] Matthew $1902, \mathrm{p} .457$ [ = Beyrichona, Conchostraca by Ulrich and Bassler 1931,p.42] Cambrian, Cape Breton.

EUCANDONA Daday $1900, \mathrm{p} .242$ for E. rostrata, E. rostrata var. thermalis, E. balatonica, E. fahaeformis, E. hungarica, E. claudiopolitana, E. pubescens, E. pubescens var. reticulata. $[=$ Candona by $G$. W. Nuller 1912,p.134].

EUCONCHOECIA [E. chierchiae] G. W. Müller 1890b,p.267

[Halocypridae-Conchoecinae by Müller 1912,p.95] Recent, off Brazil.

EUCRATERELLINA [E. randolphi] Wilson 1935,p.640. [Thlipsuridae by Wilson] Devonian, Tennessee.

EUCYPRIDAE Alm 1915,p.28, a "Gruppe" used essentially as a family to include the subfamilies: Ilyocvoringe Cyprinae and Candocyprinae.

EUCYPRIDES Sars 1925,p.110, a Group of Cypridae-Cyprinae for the genera: Cypris, Eucypris, Cypricercus, Cyprinotus, Heterocypris, Dolerocypris, Herpetocypris, Prianocypris.

EUCYPRINI Bronstein 1947,p.1C0-134, a Tribe of Cypridae-Cyprinae, to include Cypris, Eucypris, Strandesia, Dolerocypris, Isocypris. 
EUCYPRIS Vávra 1891b,p.90-106 [as a subgenus of Cypris to include Cypris (E.) pubera O.F. $N_{\bullet}$, C. (E.) fischeri Lill., $\underline{C}(\underline{E}$.) incongruens Ramdohr, $\underline{C}(\underline{E}$.$) fuscata$ (Jurine,) $\underline{C} \cdot\left(\underline{E}_{\bullet}\right)$ reticulata Zadd., $\underline{\text { C. }}$ (E.) clavata Baird, $\underline{C} \cdot\left(\underline{E}_{\bullet}\right)$ virens (Jurine) and $\underline{C} \cdot\left(\underline{E}_{\bullet}\right)$ fasciata O.F.M. Four of the above species are genotypes of other genera. Monoculus virens Jurine was designated genotype by Sars 1925,p.113. Recent, Europe; "dans I'etang de Malagnous" by Jurine.

EUCYTHERE [Cythere declivis Norman, 1865,p.16,pl.5,f.9-12 designated genotype by Brady and Norman 1889, p.178] Brady 1868b,p.429, new name for Cytheropsis Sars 1866, not M'Coy 1849 [Cytheridae-Cytherideinae by Triebel 1940,p.161] Recent, off N.W. Europe.

EUCYTHEREIS Klie 1940,p.415, [as subgenus of Cythereis, = subgenus Cythereis Skogsberg 1928,p.38. Not Jones. Two species described $\underline{C} \cdot(\underline{E} \cdot)$ mirabilis and $\underline{C} \cdot\left(\underline{E}_{\cdot}\right)$ levetzovi, plus the 14 species described by Skogsberg; genotype not indicatcd] Living off Southwest Africa and California.

EUCYTHERIDEA Bronstein 1930,p.127 [as subgerus of Cytheridea to include the two species given as Cytheridea by Sars 1925,p.158-161: c.. papillosa Bosquet 1852,

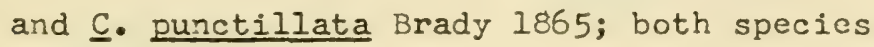
originally described as fossils and both reported 
as living in the North Atlantic. Cytheridae-Cytherideinae by Goerlich 1953,p.137].

EUCYTHERURA [ 4 species originally described; Cythere complexa Brady 1867, p.210 designated by Alexander $1936, p .692$ ] G. W. Müller 1894,p.305 [Cytheridae-Cytherurinae by Müller] Recent, British Isles. For full summary of this genus see Weingeist 1949.

EUGLYPHELLA [Strepula sigmoidalis Jones 1889c,p.576, 1890a, p.11,pl.2,f.4] Warthin 1934,p.220 [Thlipsuridae by Warthin; Quasillitidae-Ropolonellinae by Henningsmoen 1953,p.257,260,275] Devonian, New York.

EUKLOEDENELIA [E. umbilicata] Ulrich and Bassler 1923,p.313 [Kloedenellidae by Ulrich and Bassler; Kloedenellidae-Kloedenellinae by Henningsmoen 1953,p.270] Silurian, Maryland.

EUMONOPIA [E. flaveola] Claus 1891b,p.234, new name for Monopia Claus 1873 [ = Cypridina by G. W. Müller $1912, p .13]$

EUOPSIDA Daday 1900,p.207,208,305, a subtribe of Candoninae. EUPATHISTONIA Brady $1898 \mathrm{a}, \mathrm{p} .204$, nude name = Eupathistoma Brady $1898 \mathrm{~b}$. 
EUPATHISTOMA [E. natans] Brady 1898b,p.437, Bay of Bengal. $[=$ Pyrocypris by G. W. Müller 1912,p.17; both $=$ Cypridina (Cypridina) by Skogsberg 1920,p.313317].

EUPRIMITES [E. reticulogranulata] Hessland 1949,p.249

[Hollinidae-Euprimitinae by Hessland; Eurychilinidae-Euprimitiinae by Henningsmoen 1953,p.229,268] Ordovician, Sweden.

EUPRIMITIA [Primitia sanctipauli Ulrich 1894,p.652,pl.43, f. 73,74] Ulrich and Bassler 1923,p.299. [Primitiidae-Primitiinae by Bassler and Kellett 1934,p.17; Primitiidae-Eurychilininae by Kay 1940a,p.252; Hollinidae-Euprimitinae by Hessland 1949,p.243; Eurychilinidae-?Euprimitiinae by Henningsmoen 1953,p.229,268], Ordovician, Minnesota.

EUPRINITIINAE Hessland 1949,p.242, a subfamily of Hollinidae to include Euprimitia and Euprimites.

EURYCHILINA [E. reticulata] Ulrich 1889,p.52 [Beyrichiidae by Ulrich; Primitiidae-Eurychilininae by Bassler and Kellett 1934,p.20; Hollinidae-Eurychilininae by Hessland 1949,p.253; Eurychilinidae-Eurychilininae by Henningsmoen 1953,p.227,267] Ordovician, Minnesota. 
EURYCHILINIDAE Henningsmoen 1953,p.227,267, with subfamilies Eurychilininae for the genera Eurychilina, Laccochilina, Coelochilina, Apatochilina, (Platychilina Thorslund 1940, non Koken), Platybolbina, Cpikella; sof. ?Euprimitilnae for the genera Euprimitia, ?Primitiella, ?Euprimites, ?Haploprimitia, ?Ectoprimitia, ?Laccoprimitia; and s.f. ?Primitiopsinae for Primitiopsis.

EURYCHILININAE Ulrich and Bassler 1923,p.302, a subfamily of Primitiidae, for the genera Eurychilina, Coelochilina, Chilobolbina, Apatochilina, Apatobolbina.

EUCYPRIDAE Alm 1915,p.28-31, A "Gruppe" used in same sense as Family, to include the subfamilies Ilyocyprinae, Cyprinae, and Candocyprinae. See also Furtos 1933, p. 425,426 .

EUCYPRIDES Sars 1925,p.110, a Group of Cypridae-Cyprinae for the genera Cypris, Eucypris, Cypricercus, Cyprinotus, Heterocypris, Dolerocypris, Herpetocypris, Prionocypris.

EURYCYPRIS [E. latissima] G. W. Müller 1898, p.263, Recent Madagascar. $[=$ Cypris 0 . F. Müller, by G. W. Müller $1912, \mathrm{p} \cdot 178]$. 
EURYPYLUS [E. petrosus] Brady 1869, Fonds de la Ner,p.141, Recent off Cape Verde. [ = Sarsiella by G. W. Müller $1912, \mathrm{p} \cdot 42$.

EUSTAPHANELLA Swartz and Swain 1942,p.674, new name for Eustephanus Swartz and Swain, not Reichenbach, 1849 [Thlipsuridae by Henningsmoen 1953, p.250].

EUSTEPHANUS [E. catastephanes] Swartz and Swain 1941,p.435 non Reichenbach, 1849 [Thlipsuridae by SES] Devonian, Pernsylvania. [renamed Eustaphanella by Sirs $1942, \mathrm{p} \cdot 674 \cdot]$

EVLANCLLA Egerov 1950,p.63, by Polenova 1952,p.89 [Genotype E. laschenkoi Egarov 1950] Kloedenellidae--

Lichwininae by Polenova. Devonian to ?Permian by Polenova.

EXOPHTHAIN:OCYTHERE [E. mamillata] Triebel 1938b, p.136 [Cytheridae by Triebel] Cretaceous, Germany.

$$
\text { F }
$$

FABALICYPRIS [F. wileyensis] Cooper 1946,p.59,60 [Sairdiidae by Cooper] Pennsylvanian of Illinois.

FABERIA [F. anomala] Niller 1889,p.549, Ordovician, Ohio. [Not an ostracod, ..."the phosphatized cast of a small species of Ctenodonta, a pelecypod from the 
Arnheim formation," by Bassler and Kellett 1934, p.317.]

FALjIPOLLEX [F. altituberculatus] Kesling and McMillan 1951, p.67 [Hollinidae by KEM] Devonian, Michigan.

FAVILLA [F. puelia] Coryell and Fields 1937,p.8; revised by Edwards 1944,p.523-521+ [Cytheridae-Cytherideinae by CGF] Miocene, Panama. [Preoccupied by Favella Jörgensen 1925, Prot., fide Neave, Nomenclator Zoologicus vol.2,p.401.] = Puriana Coryell and Fields by Coryell, in Puri 1953b, p.751.

FAVULELlA [Bythocypris favulosa Jones 1889a,p.338,pl., figs.1,2a-c] Swartz and Swain 1941,p.438 [?Thlipsuridae by Swartz and Swain] Devonian, Pennsylvania.

FIMBRIA Neviani 1928,p.72, a "Gruppo", a subgenus or genus of "Cythereis", but preoccupied many times by such as Fimbria Bohadsch 1761, Mollusca, Fimbria Rosso 1826, Tunicata etc., fide Neave, Nomenclator Zoologicus, vol.2, p.408.

FLEXUS Neviani 1928,p.72,75, as "Gruppo" = subgenus of Cythereis. Subgenotype: Cythere plicata Munster $1830, \mathrm{p} \cdot 63$. 
FOSSIRICHTERINA [Richterina (Fossirichterina) intercostata] Matern 1929, 0.70 [As a subgenus of Richterina by Matern; Entomidae by Bassler and Kellett 1934. P.467; Entomozoacea-Entomozoidae-Entomozoinae by Pribyl 1951,p.105] Devonian, Germany.

FRANKLINELLA [F. novecosta] Stewart and Hendrix 1939,p.1989, nom.nud.; Stewart and Hendrix 19450,p.10?. iGlyptopleuridae by SEH; Kirkbyidae by Henningsmoer 1953,p.273; Entomozoacea-Entomozoidae-Entomozoinae by Pribyl 1951,p.105] Devonian, Ohio. [non Franklinella (n•n., pro Physapus Leach 1815 ) Karny 1910, Thysanopt., fide Neave 1939, vol.2,p.421].

G

GEFFENINA [G. marmerae] Coryell and Sohn 1938, p.599

[Kloedenellidae by CES; KloedeneILidae-Kloedenellinae by Henningsmoen 1953,0.243, 271] Mississippian of West Virginia.

GEFFENITES [G. jungae] Coryell and John 1938,p.600

[Kloedenellidae by CES; Kloedenellidae-Kloedenellinae by Henningsmoen $1953, n .243,2711$ Mississippian of West Virginia.

GEISINA [Beyrichiella gregaria Ulrich and Bassler 1906,p.157, pl.11,f.18] Johnson 1936,p.21. [Kloedenellidae by Johnson; Kloedenellidae-Kloedenellinae by 
Henningsmoen 1953,p.242,271] Pennsylvanian, Missouri.

GIBBA Fuchs 1920,p.81-83, a subgenus of Beyrichia created for Reyrichia (Gibba) spinosa Fuchs n.sp., and Beyrichia tetrapleura Fuchs 1915,p.77,pl.18, f.11-13; both from Devonian of Germany $\underline{B} \cdot(\underline{G} \cdot)$ spinosa has by later authors been transferred to Kloedenia, but the name is invalid as it is a junior homonym of B. spinosa (Hall) Hall 1859; and B. spinosa (Ulrich) Miller 1897. B. tetrapleura by elimination must be the subgenotype.

GIGANTOCYPRIS [G. agassizii,p.155, G. pellucida,p.164] G. W. Müller 1895,p.164 [Cypridinidae-Cypridininae by Müller 1912,p.23] Recent, W. Coast of Central America. By describing G. agassizii first, before descrjbing the genus, Miller clearly thought of it as the type.

GILIINA [G. vitharri] Coryell and Johnson 1939,p.217 [Kirkbyidae by CEJ; Kloedenellidae by Agnew 1942, p.758; Kloedenellidae-Kloedenellinae by Henningsmoen 1953,p.242,270] Mississippian of Illinois.

GIRTYITES [G. spinosus] Coryell and Booth 1933,p.261

[Kirkbyidae by CEB] Pennsylvanian of Texas. [Not Girtyites Wedekind 1914, and renamed Kegelites by 
Coryell and Booth 1933, on second page of table of contents of vol.14 of Amer.Midl.Naturalist.] The genotype $=$ a young molt of Amphissites dattonensis Harlton, by Kellett $1936, p .773$.

GLOBOCYPRIS [G. trisetosa] Klie 1939,p.1I] [Cypridae by KIie] Recent, Kenya Colony.

GLOSSOMORPHITES Hessland $1954, \mathrm{p} .227$, nom.nov., for Glossopsis Hessland 1949, non Busch 1904, an annelid.

GLOSSOMORPHITINAE Hessland 1954,p.227, nom.nov., for GIOSSOpsinae Henningsmoen 1953 to correspond with necessary change in generic name.

GLOSSOPSIINAE Henningsmoen 1953,p.201,266, a subfamily of Sigmoopsiidae, for the genera Glossopsis and Aulacopsis. [Glossopsis Hessland 1949 being preoccupied; = Glossomorphitinae by Hessland 1954, p.227.

GLOSSOPSIS [G. Iingua] Hessland 1949,p.296 [Hollinidae-Tetradellinae by Hessland; Sigmoopsiidae-Glossopsiinae by Henningsmoen 1953,p.201,266] Ordovician, Sweden. [not GIossopsis Busch 1940,p.225-226, an annelid; = Glossomorphites Hessland 1954,p.227, nom. nov.] 
GLYPTOBAIRDIA [G. bermudezi] Stephenson 1946b,p.345

[Bairdiidae by Stephenson] Recent, Cuba.

$[=$ Triebelina Van den Bold 1946, whose description preceded it by only a few days].

GLYPTOPLEURA [G. inopinata] Girty 1910,p.236 [Glyptopleuridae by Girty] Pennsylvanian, Arkansas.

GLYPTOPLEURIDAE Girty 1910,p.234 for the genus Glyptopleura.

GLYPTOPLEURI:A [G. montifera] Coryel] 1928b,p.38] [Glyptopleuridae hy Coryell; Kloedenellidae-Glyptopleurinae by Henningsmoen 1953,p.272] Pennsylvanian, Oklahoma.

GLYPTOPLEURINAE Henningsmoen 1953,p.197,225,272, a reduction of Girty's family to a subfamily of Kloedenellidae to include Glyptopleura, Glyptopleurina, Glyptopleuroides, Nesoglypha, Venula, Svantovites, and ?Varix.

GLYPTOPLEURTTES [G. tyri] Coryell and Johnson 1939,p.219

[Glyptopleuridae by CGJ] Nississippian of Illinois. $[=$ Glyptopleura by Cooper 1941,p.42.]

Girptcpleuroides [G. insculptus] Corneis and Gale 1939, P.283 [Glyptopleuridae by CEG; KIoedenellidae-Glyptopleurinae by Henningsmoen 1953,p.272] Mississippian of Illinois. 
GOLCONDEILA [G. sulcata] Croneis and Gale 1939,p.262 [Drepanellidae by CEG] Mississippian of Illinois.

GOMPHOCYTHERE [Limnicythere obtusata Sars 1910,p.754,pl.7k, f.8-14] Sars 1924a,p.174 [Cytheridae-Limnicytherinae by Sars 1925,p.148; Cytheridae-Xestoleberinae by Martin 1940,p.337-340] Recent, Lake Victoria Nyanza.

GONIOCYPRIS [G. mitra] Rrady and Robertson $1870, \mathrm{p} .15$ [ = the fry of Anodonta cygnea by Brady and Norman 1889, p. 120] Recent, England.

GRAPHIADACTYLIIS [Kirkbya lindahli arkansana Girty 1910, p.231 = Graphiodactyllis arkansana Roth 1929a, p.10] Roth 1929a,p.10 [Graphiodactylidae by Kellett 1936,p.773; Quasillitidae-Quasillitinae by Henningsmoen 1953,p.256,257,275] Mississippian of Arkansas.

GRAPHIODACTYLIDAE Kellett [December] 1936,p.773, a family to include Graphiodactylus, Waylandella, Coryellites, Healdia. [ = Quasillitidae Coryell and Malkin [November] 1936, by Henningsmoen 1953,p.256. GRAPHIODACTYIUS Roth 1929b,p.292, an attempted change in spelling of Graphiadactyllis Roth 1929a, which is not permitted by the Rules, according to Moore, 
Weller and Knight 1942,p.256,257. See Roth 1929c for full description of genus.

GRAVIA [Gravia(Gravia)aculeata, and Gravia(Gravia)volgaensis] Polenova 1952,p.83 [Acronotellidae-Graviinae by Polenova] Devonian, USSR. [compared to Monoceratella, Tricornina and Saccelatia] G. aculeata was not figured; $G_{\cdot}\left(\underline{G}_{\cdot}\right)$ volgaensis, described p.85,pl.2, f.4.

GRAVIINAE Polenova 1952,p.82, a subfamily of Acronotellidae, for the genus Gravia, with subgenera Gravia and Russia [and apparently for Selebratina, Sulcatia, and Lunularia] Silurian to Carboniferous.

GUTSCHICKIA [Whipplella ninevehensis Holland 1934,p.345,pl.25, f.3a-c] Scott 1944a,p.146. [Cypridae by Scott] Permian of Pennsylvania.

HABROCYTHERE [H. fragilis] Triebel 1940,p.165 [Cytheridae-Cytherideinae by Triebel] Cretaceous, Germany.

HALLATIA [H. healeyensis] Kay 1934,p.335 [Primitiidae-Eurychilininae by Kay 1940a,p.253; Drepanellidae-Ulrichiinae by Schmidt 1941,p.51; Beyrichiacea of uncertain family by Henningsmoen 1953, p.248, 273] Ordovician, Ontario. 
HALIIFLIA [H. retifera, designated by Miller 1892,p.707, fide Warthin 1948,p.646] Ulrich 1891,p.184 [Beyrichiidae by Ulrich 1894,0.632; Primitiidae-Primitinae by Bassler and Kellett 1934,p.17; Primitidae-Eurychilininae by Kay 1940a,p.253; Hollinidae by Pokorný 1950,p.588; Beyrichiacea of uncertain family by Henningsmoen 1953,p.229, 248,273] Devonian, Kentucky•

HALOCYPRIA [H. globosa] Claus 1874a,p.7; 1874b,p.178 [Halocypridae by Claus] Recent, Atlantic Ocean.

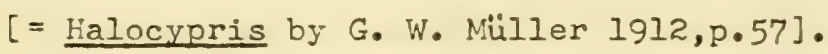

HALOCYPRIDAE Dana 1853,p.1281, as a family of the Tribe Cyproidea $[=$ Ostracoda], divided into Subfam. Cypridininae for the genus Cypridina; and Subfam. Halocyprinae for Halocypris, and Conchoecia.

HALOCYPRIFORMES Skogsberg 1920,p.158,555-756, a Suborder for the family Halocypridae.

HALOCYPRINAE Dana 1853,p.281, a subfamily of Halocypridae for Conchoecia and Halocypris.

HALOCYPRIS [Conchoecia inflata and C. brevirostris Dana $1849, \mathrm{p} \cdot 52 ;$ both $=$ Halocypris inflata (Dana) by G. W. Müller 1912,p.58] Dana 1853,p.1301. [Halocypridae-Halocyprinae by Dana] Recent, Pacific Ocean. 
HAN.ILTONELLA [Leperditia punctulifera Hall 1860,p.92]

Stewart 1936,p.756 [Bairdiidae by Stewart;

Cytherellidae by Agnew 1942,p.758] Devonian, New

York [Stewart has previously identified this

species as Cytherella (?) bispinulatus Stewart

$1927, \mathrm{p} \cdot 60, \mathrm{pl} \cdot 5, \mathrm{f} \cdot 18,19$, which was made the geno-

type of Ponderodictya Coryell and Malkin 1936,

earlier in the same year. = Ponderodictya.]

HANAITES [kalliella (Hanaites) givetiana] Pokorny 1950,

p.537,599 [Hollinidae, and subgenus of Halliella by Pokorný] Devonian of Czechoslovakia.

HAPLOCYTHERIDEA [Cytheridea montgomeryensis Howe and Chambers 1935,p.17,pl.1,f.1;pl.2,f.1-3,7,9;pl.6,f.17,18]

Stephenson 1936,p.700 [as subgenus of Cytheridea by Stephenson; as genus by Stephenson 1946; Cytheridae-Cytherideinae by Goerlich 1953,p.138] Eocene of Iouisiana.

HAPLOPRINITIA [Primitia minutissima Ulrich 1894,p.651,pl.45, f.31] Ulrich and Bassler 1923,p.297 [Primitiidae by UEB; Primitiidae-Primitiinae by Bouček 1935 , p.43; Eurychilinidae-?Euprimitiinae by Henningsmoen 1953,p.229,268 J Ordovician of Minnesota.

HARDINIA [H. concava] Coryell and Rosanski 1942,p.146

[?Youngiellidae by CGR] Mississippian of Illinois. 
HARLTONELLA [H. ardmorensis] Bradfield 1935,p.118 [Bairdiidae by Bradfield] Pennsylvanian of Oklahoma.

$[=$ Waylandella by Cooper $1946, p .63]$.

HASTACYPRIS [H. bradyi] Croneis and Gutke 1939,p.60

[Bairdiidae by CGG] Mississippian of Il]inois.

HASTIFABA [H. spinosa] Cooper 1946,p.1] [KIoedenellidae by Cooper; Kloedenellidae-Kloedenellinae by Henningsmoen 1953,0.242,271] Pennsylvanian of Illinois.

HAWORTHINA [Bairdia bulleta Harris and Lalicker 1932,p.404, pl.37,f.7] Kellett 1935,p.161 [Cypridae by Kellett; Bairdildae by Agnew 1042,p.758] Permian of Texas.

HEALDIA [H. simplex] Roundy $1926,0.8$ [Healdidae by Harlton 1933,p.26; Bairdiidae by Rassler and Kellett 1934, p. 324; Healdildae of the Platycopa by Triebel 1950,p.117] Pennsylvanian of Texas.

HEALDIACYPRIS [H. perplexa] Bradfield 1935,p.103 [Bairdiidae by Bradfield] Pennsylvanian of Oklahoma.

HEALDIANEILA [H. damwinulinoides] Posner 1951,p.75, fide Polenova 1952,p.121 [Healdiidae by Polenova] who gives range Devonian to Carboniferous. 
HEALDIIDAE Harlton 1933,p.26, a Family to include Healdia, Seminolites, Cavellina. This family placed in Platycopa by Triebel 1950b,p.117, and by Henningsmoen 1953,p.259-261, who places here Healdia, Cavellina, Ponderodictya, Healdioides, and possibly here: Cribroconcha, Incisurella, Paracavellina, Platychilella, Seminolites, Sulcella, Tetratylus.

HEALDIINAE Přibyl and Šnajdr 1950,p.125,168, a subfamily of Bairdiidae for the genus Healdia.

HEALDIOIDES [H. diversus] Coryell and Rosanski 1942,p.148 [Healdildae by CER; Bairdiidae by Agnew 1942, p.758; Healdiidae by Henningsmoen 1953,p.261] Mississippian of Illinois.

HEMIAECHMINOIDES [H. monospinus] Morris and Hill 1952,p.7, 8 [Aparchitidae by NGH; Beyrichiacea of uncertain family by Henningsmoen 1953,p.273. Silurian, Tennessee.

HEMICYPRIS [3 species: H. pyxidata (Moniez); H. Ovata Sars; H. megalops Sars] Sars 1903,p.24 [all 3 species = Cyprinotus by Nïller 1912; the first and last $=$ Heterocypris by Klie, 1932,p.471; 1938,p.23.] The first species which appears to have been the one Sars intended as most characteristic was from Sumatra. 
HEMICYTHERE [ 9 species, the first of which, Cythere villosa Sars $1866, p .42$ was designated genotype by Edwards 1944,p.517-518] Sars 1925,p.182 [Cytheridae-Cytherinae by Sars] Recent, North Atlantic.

HEMICYTHERIDEA [H, reticulata] Kingma 1948,p.70 [Cytheridae-Cytherideinae by Kingma] Pliocene, Java and Sumatra.

HEMICYTHERIDEIS [Cytheridea elongata Brady 1868b,p.421, pl.28,f.13-16,pl.40,f.6] Ruggieri 1952,p.62. [Cytheridae] Recent, British Isles. [Very close to Cushmanidea and also Pontocypris]

HEMICYTHERURA [Cythere cellulosa Norman 1865,p.22,pl.5, f.17-20; pl.6,f.17] Elofson 1941,p.314 (As a subgenus of Cytheropteron, by Elofson; Cytheridae-Cytherurinae by Hornibrook 1952,p.58] Recent, North Atlantic.

HEMIKRITHE [H. orientalis] Van den Bold 1950,p.901-904 [Cytheridae by Van den Bold] Recent, Indo-Pacific.

HERPETOCYPRELLA [H. mongolica] Daday 1909,p.14 [Cypridae] Recent, Issyk-Kul, Turkestan.

HERPETOCYPRELIINI Bronstein, 1947,P.150, a Tribe of Cypridae-Cyprinae for Herpetocyprella Daday. 
HERPETOCYPRIDINAE Kaufmann 1900a,p.105; 1900h,p.261. a subfamily of Cypridae to include: Herpetocypris Iyodromus, Prionocypris, Microcypris.

HERPETOCYPRINI Bronstein 1947,p.141-148, a Tribe of Cypridae-Cyprinae to include: Ilyodromus, Stenocypria, Herpetocypris, Stenocypris.

HERPETOCYPRIS Sars $1889 \mathrm{a}, \mathrm{p} \cdot 34$, a change in the spelling of Erpetocypris Brady and Norman, for which he gives no explanation. Claus, 1893,p.198 in a footnote does likewise. Brady and Norman 1896,p.772 in a footnote indicate: "In Part I, for Erpetocypris read partim Hernetocypris." [According to Moore, Weller and Knight 1942, this change, even by the authors, is not in keeping with the Rules. In this case it makes little difference for the genotype of Erpetocypris or "Herpetocypris," Cypris reptans Baird, was designated the genotype of Candona by Baird 1846,p.414. Herice "Herpetocypris" = Candoria. $]$

HERR:ANNELLA [H. waldschmidti] Paeckelmann 1922,p.15-17 [as a subgenus of Leperditia by P.] Devonian, Germany. [Not Herrmannella Canu 1891, a Copepod]. 
HERRMANNINA Kegel. 1934,p.409, new name for Hermannella. Paeckelmann 1922 [as a genus by Swartz 1949, p.314, of Leperditildae-Leperditilnae].

HESPERIDELLA [Primitia estonica Bonnema 1909,p.32,p1.6, f.30] Öpik 1937,p.113 [Piretellidae by Op1k; Hollinidae-Hollinae by Schmidt 194l, p.32; Tetradellidae-Bassleratinae by Henningsmoen 1953, p.267] Ordovician, Estonia.

HESSLANDELLA [Ctenentoma macroreticulata Hessland 1949, $p .269, p 1.6, f \cdot 13,15-17$ ] Henningsmoen $1953, p .215$ [Tetradellidae-Tetradellinae by Henningsmoen 1953,p.267] Ordorician, Sweden.

HESYECHILUS Brady 1875, Fonds, vol.2, pt..1, chap.2,p.11, for Hesvechilus enntortus (Noman) = Sclerochilus contortus (Noman)? [in a list, with no description and probably a typographical error].

HETEROCHILINA [H. obIiqua] :oulsen $1937, \mathrm{p} .59-60$ [Leperditiidae-Leperditiinae by Henningsmoen 1953, n.274] Ordovician, Greenland.

HETEROCYPR.IDEIS [Cythere (Cytheridea) sorbyana Jones 1857, p.44,pl.4,f.6a-e] Elofson 1941,p.258: CytheridaeCytherideinae by Elofson] Pleistocene, Yorkshire, England; Iiving North Atlantic. 
HETEROCYPRIS [Cypris incongruens Ramdohr 1808,p.86,pl.3, f.1-12,15-20] Claus 1893,p.198 [Cypridae-Cyprinae-Eucyprides by Sars 1925,p.124] Recent Europe, also Asia, Africa, North and South America.

HETEROCYTHEREIS [Cythere albomaculata Baird 1838,p.142, pl.5,f.23] Elofson 1941,p.280,292,295 [as a subgenus of Cythereis by Elofson] Recent, North Atlantic.

HETERODESMUS [H. adamsii] Brady 1866,p.387 [?=Codonocera by Brady 1902,p.189; Incertae sedis by G. W. Müller $1912, p \cdot 398]$

HIBBARDIA [Amphissites lacrimosus Swartz and Oriel 1948, p.553,pl.79,f.15] Kesling 1953d,p.20 [Beyrichiidae by Kesling] Devonian, New York.

HILBOLDTINA [H. multiplicata] Scott and Summerson 1943, p.669 [Cæpridae by SES] Pennsylvanian, Tennessee.

HIPPA [H.latens] Barrande 1872,p.516 [Entomidae by Ulrich 1894,p.632; Beyrichildae by Bassler and Kellett 1934,p.328; Primitiidae by Swartz 1936,p.550; Primitidae-?Primitiinae by Schmidt 1941,p.25, and also Hollinidae-?Ctenentominidae by Schmidt 1941,p.34; Beyrichiacea, of uncertain family by Henningsmoen 1953,p.249,273] Ordovician, Bohemia. [Not Hippa Fabricius 1787; = Trubinel]a Priby] $1950, p .102$, new name ] 
HIPPONICHARION [H. eos] Natthew $1886, p .64$, Cambrian New Brunswick [placed in Conchostraca by Ulrich and Bassler 1931,p.62.

HIRSCHMANNIA [Cythere viridis 0.F.Müller 1785,p.64,pl.7, f.1,2] Elofson 1941,p.331 [Cytheridae] Recent, North Atlantic.

HIRSUTOCYTHERE [H. hornotina] Howe 1951b,p.21 [Cytheridae-Cytherinae by Howe] Eocene, Florida.

HOGMOCHILINA [Isochilina (Hogmochilina) elliptica] Solle 1935,p.20 [As subgenus of Isochilina by Solle; Leperditiidae-Isochilininae by Swartz 1949,p.324] Devonian, Sptizbergen.

HOIIINA [Ctenobolbina insolens Ulrich IC00,p.182,pl.8, f.10, 11] Ulrich and Bassler 1908,i.315 [Beyrichiidae by Ulrich and Bassler 1923,p.310; Hollinidae-Hollinae by Schmidt 1941,p.32] Devonian, Kentucky. HOLIINELIA [H. dentata] Coryel] 1928b,p.377-378 [Beyrichiidae by Bassler and Kellett 1934,p.331; Hollinidae by Swartz 1936,p.551; Hollinidae-Hollinae by Schmidt 1941,p.32] Pennsylvanian, Oklahoma.

HOILINIDAE Swartz 1936,p.551, a family to include Ctenobolbina, Parabolbina, Hollina, Hollinella, Janischewskya, Neochilina. [This family materially revised by 
Henningsmoen 1953,p.197,239,270. As revised,

Henningsmoen includes: Hollina, Hollinella,

Falsipolex, Parabolbina, Proplectrum, Hanaites,

Subligaculus, Ctenoloculina, Tetrasacculus,

Bisacculus, Janischewskya, and ?Sulcicuneus as

valid genera, and a number of others as synonyms

of these.

HOLLININAE Schmidt 1941,p.32 [=Hollinidae of Swartz 1936 t Pirctellidae of Öpik 1937, but is discarded by Henningsmoen 1953,p.239,279] Schmidt includes Hollina, Hollinella, Tanischewskya, Bromidella, Piretella, and Hesperidella.

HOLIITES [H. papillosus] Coryell and Sample 1932,p.252 [Beyrichiidae by CES; = an early molt of a Hollinclla by Kellett 1933,p.69] Pennsylvanian, Texas.

HOLTEDAHLINA [H. tcres] Solle 1935,p.46 [Leperditiidae by Solle] Devonian, Spitzbergen. [Not Holtedahlina Foerste 1924, Brach. fide Neave 1939, vol2,p.678].

HOLTEDAHLITES Solle $1936, \mathrm{p} .282$, new name for Holtedahlina Solle 1935. [Leperditidae-Leperditinae by Henningsmoen $1953, \mathrm{p} \cdot 274$.

HOMOCYPRIS [H. conoidea] Sars 1924a,p.119 [Cypridae by Sars] Raised from dried South African mud. 
HUiGARELLA Méhes 1911,p.22, a suggested genus for Bairdia

(?) problematica Daday, n.sp., Triassic, Hungary. HUNGAROCYPRINI Bronstein 1947,p.149, a Tribe of Cypridae-Cyprinae for the genus Hungarccypris Vavra. HUNGAROCYPRIS [2 species: Cypris dispar Chyzer 1858 (not S. Fischer) = H. madarászi örley, and H. gawemülleri, n.sp.] Vávra 1906a,p.416 [Cypriade by Vávra; Cypridae-Cyprinae-Hungarocyprini, by Bronstein 1947] H. madaraszi Recent, Hungary and the largest ostracod in Europe; $\underline{H}$. gawemülleri Recent, Sumatra. [Daday's 1895 description of Cyprois dispar $=$ H. madaraszi is one of the most complete for any ostracod.]

HUTSn:IA [F. vulgaris] Swain 1946a,p.123 [Cytheridae by Swain, near Cytheropteron, Xestoleberis, and Orthonotacythere] Jurassic, Louisiana well. IYALOCYPRIS [H. africana] Brady 1913b,p.461 [= Isocypris Müller, by Sars 1924,p.139] Recent, Orange Free State.

HYPHASMOPHORA [H. textiligera] Van Pelt 1933,p.339 (Thlipsuridae by Van Pelt] Devonian, Michigan. 
HYPOTETRAGONA [H. impolita] Morey 1935,p.326 [Cytherellidae by Morey; Primitiidae by Agnew 1942,p.759; Beyrichiacea of uncertain family by Henningsmoen 1953,p.273] Mississippian of Missouri.

IDIOMORPHA [I. ornata] Croneis and Gale 1939,p.284

[Glyptopleuridae by CEG] Mississippian of Illinois. [Not Idiomorpha Förster $1863=$ Idiomorphina Croneis and Gale, new name].

IDIOMORPHINA Croneis and Gale, new name, in Croneis June 15, 1939b; and Croneis and Gale July 1939, for Idiomorpha Croneis and Gale 1938. ["Glyptopleurina Coryel1 1928, by Cooper 1941,p.42].

IIIOCYPRIS Daday 1900,p.229 = Ilyocypris Brady and Norman 1889.

ILIOCYPREILA [I. gibba repens (Vávra) = Ilyocypris bradyi Sars 1890,p.59] Daday 1900,p.237 [= Ilyocypris by G. W. Müller 1912,p.154 and by many others, but recognized as good by Sars 1925,p.105, and by Hessland 1949,p.139,149,p1.4. Cypridae-Candoninae-Synopsida by Daday] Recent, Norway-Sweden.

ILYOBATES [I. praetexta] Sars 1866,p.59 [Cytheridae by Sars] Recent, Norway [Not Ilyobates Kraatz, 1858, col. = Krithe Brady, Crosskey and Robertson 1874]. 
ILYOCYPREILA Sars 1925,p.105, and by others = Iliocyprella Daday 1900.

ILYOCYPRIDES Sars 1925,p.105, a Group of Cypridae-Cyprinae for Ilyocypris and Ilyocyprella.

ILYOCYPRIDINAE Kaufmann 1900a,p.107; 1900b,p.341, a subfamily of Cypridae for the genus Ilyocypris.

ILYOCYPRINAE Masi 1906a,p.133-146 = Ilyocypridinae Kaufmann 1900, and Jiliocyprinae Muller 1900.

IIYOCYPRIS [Cypris gibba Ramdohr 1808,p.91,pl.3,f.13-17]

Brady and Norman 1889,p.106 [Cypridae-Ilyocypridinae by Kaufmann 1900a,p.107] Recent, Europe.

ILYOCYPROIS [Ilyocypris gibba tuberculata Kertész 1893, p.170,pl.6,f.1-12] Masi 1906a,p.261 _ = Ilyocypris by G. W. Muller 1912,p.153].

ILYOCYTHERE [I. gibba] Klie 1939b,p.364 [Cytheridae-Cytherinae, near Leptocythere by KIie] Brackish water, Brazil.

ILYODROMUS [ 7 species, of which the first Candona stanleyana King 1855,p.66,pl.10,f.H should be the type] Sars $1894, \mathrm{p} \cdot 38,39, \mathrm{pl} \cdot 5, \mathrm{f} \cdot 3 \mathrm{a}-\mathrm{e}$ [Herpetocypridinae by Kaufmann 1900,a,p.105] Recent, Australia. 
INCISURELLA [I. prima] Cooper 1941,p.32 [Bairdiidae by Cooper; ?Healdiidae by Henningsmoen 1953,p.260] Mississippian of Illinois.

INDIANA [3 species: I.ovalis, I.prima, I.lippa; with I. lippa selected genotype by Ulrich and Bassler 1931,p.68-70] Natthew 1902,p.460 [Conchostraca by Ulrich and Bassler; Branchiopods by Bassler and Kellett 1934,p.336] Cambrian, Cape Breton.

ISOCHILINA [ 2 species, of which Leperditia (Isochilina) ottawa was designated by Bassler and Kellett 1934,p.336] Jones 1858a,p.248; 1858c,p.97 [Leperditiidae-Isochilininae by Swartz 1949, p.32l] Ordovician, Canada.

ISOCHILINIA Vogdes 1893,p.102, error for Isochilina Jones 1858.

ISOCHILININAE Swartz 1949,p.321, a subfamily of Leperditiidae for the genera: Isochilina, Hogmochilina, Teichochilina, Dihogmochilina, Saffordellina.

ISOCYPRIS [I. perangusta, I. priomena] G. W. Müller, 1909, p.159 [Cypridae-Cyprinae by Müller] Recent,South Africa.

ISOCYTHERE [I. nova] Terquem 1885,p.43 [Platycopa-Cytherellidae by Terquem] Jurassic, France. 
ISOCYTHEREIS [I, fissicostis] Triebe] 1940,p.208 [Cytheridae-Cytherinae by Triebel] Albian, Germany •

JANETINA [J. harrietensis] Coryell and Malkin 1936,p.19 [Quasillitidae by CEM; GraphiadactyIIidae by Agnew 1942,p.759; Quasillitidae-Quasillitinae by Henningsmoen 1953,p.256] Devonian of Ontario.

JANISCHEWSKYA [J. digitata] Batalina 1926, p.1332,1336 [Beyrichiidae by Bassler and Kellett 1934,p.342; Hollinidae by Swartz 1936,p.551] Carboniferous, Russia.

JAI:USELLA [J. biceratina] Roth 1929d,p.363 [Beecherelidae by Roth] Devonian, Oklahoma.

IAVANELIA [J. kendengensis] Kingma 1945, p. E, $i=$ Pel]ucistoma by Van den Bold 1950,p.86] Pliocene, Java.

JEHNI!GiINA [Graphiodactylus catenulatus Van Pelt 1933 , p.333,pl.39,f.31,32] Coryell and Malkin 1936,p.19 [Quasillitidae by CEM; Quasillitidae-Quasillitinae by Henningsmoen 1953,p.226,275] Devonian of Ontario.

JIIOCYPRIS G. W. Müller 1900, p.87 = Ilyocypris JIIOCYPRINAE G: W. Müller $1900, \mathrm{p} .87$ = Ilyocypridinae Kaufmann 1900. 
JONESELLA [Leperditia crepidiformis Ulrich 1879,p.10,pl.7, f.3,3a] Ulrich 1890,p.121 [Beyrichiidae by Ulrich; Primitidae by Bassler and Kellett 1934, p.343; Drepanellidae-Drepanellinae by Schmidt 1941,p.50] Ordovician, Kentucky, Ohio.

JONESIA [Cythere simplex Norman 1865,p.17,pl.5,f.1-4] Brady 1866,p.362 [Cytheridae-Bythocytherinae] Recent, Shetlands.

JONESINA [Beyrichia fastigiata Jones and Kirkby 1867 , p.220] Ulrich and Bassler 1908,p.324 [Beyrichildae-Kloedenellinae by UEB; Kloedenellidae by Bassler and Kellett 1934,P.30; Kloedenellidae-Kloedenellinae by Henningsmoen 1953,p.242-270] Carboniferous, Scotland.

JONESITES [Primitia excavata Jones and Holl 1869, p.222, pl.15,f.10a-c] Coryell 1930,p.294-295, new name for Placentula Jones and Holl. [Primitildae by Bassler \& Kellett 1934,p.17-19; ?Drepanellidae-Ulrichiinae by Schmidt 1941,p.51; Drepanellidae-?Bolliinae by Henningsmoen 1953,p.268] Silurian, England.

JUGOSAE Jones $1855 \mathrm{a}, \mathrm{p} .85$, a Group of species of the genus Beyrichia M'Coy. 
KALUGIA Egerov 1950, compared to Evlanella by Polenova $1952, \mathrm{p} \cdot 90$.

KANGARINA [K. quellita] Coryell and Fields 1937,p.12 [Cytheridae-Cytherurinae by CEF] Niocene, Panama.

KARISTEINELLA [K. reticulata] Boulek 1936,p.67 [Beyrichiidae by Boukek] Silurian, Bohemia.

KEGELITES Coryell and Booth 1933, 2nd page of table of contents of Amer.Midland Naturalist, vol.14, new name for Girtyites CEB, $\mathrm{p} .261$ of same volume. $[=$ Amphissites by Kellett 1936,p.772; by Agnew 1942, p.759].

KELLETTLLIA [K. naviculata] Delo 1930,p.176 [Cytheridae? by Delo; Kirkbyidae by Bassler and Kellett 1934, p.33-34; Cytheridae by Kellett 1935,p.159; Kloedene]lidae-Kloedenellinae by Henningsmoen 1953,p.271] Carboniferous, Texas.

KELLETTIMA [Ulrichia rohusta Kellett 1933,p.92,pl.15,f.3340,42] Swartz 1936,p.585 [Kirkbyidae by Swartz] Permian of Kansas. 
KIF,30WIA [Reyrichia dissecta Krause 1892,p.392,p1.21,f.3] Ulrich and Bassler 1908,p.306-307 [as a subgenus of Tetradella by UEB; Beyrichiidae by Bassler and Kellett 1934,p.27; Tetradellidae by Swartz 1936,p.551; Primitiidae-Bolliinae by Kay 1940a, p.259; Hollinidae-Tetradellinae by Schmidt 1941, p.37; Sigmoopsiidae-Sigmoopsiinae by Henningsmoen 1953,p.210-266] Ordovician drift, Germany.

KTWNEKLLIEA [K. waerni] Eenningsmoen 194?,p.412. [DrepanellidacDrepanellinae by $\mathrm{H}$ : Drepanellidae-?Bolliinae by Henningsmoen 1953,p.268] Ordovician, Sweden.

KIRKFIJIDAL Spizharsky 1939,p.195 = Kirkbyidae Ulrich and Basslor 1906 .

KIRKBYA [Dithyrocaris permiana Jones $1850 \mathrm{~b}, \mathrm{p} .66, \mathrm{p}] .1 .8, f .1$ ] Innes in Kirkby 1860,p.129 [Beyrichiidae hy Ulrich 1894, p.632; Kirkbyidae by Bassler and Kellett 1934,p.350] Permian, England.

FIRKYYILA [K. typa] Coryell and Booth 1933,p.252 [Kirkbyidae by CGB; Kloedenellidae-Kloedenellinae by Itenningsmoen 1953,p.243,245,271] Pennsylvanian of Texas.

KIRKBYETI.INA [K. styliolata] Kummercw 1939,p.30 [Kirkhyidae by Kunmerow; Incertae Sedis by Acnew 1942,p.219] Lower Carboniferous, Germany. 
KIRKBYIA Cossmann, 1899,p.45, new name for Synaphe Jones and Kirkby 1898, not Synaphe Hubn. Lepid. 1816. $[=$ Beyrichiella Jones and Kirkby by Bassler and Kellett 1934,p.211,212].

KIRKBYIDAE Ulrich and Bassler 1906,p.159, a new family for the genus Kirkbya.

KIRKBYIDAE Girty 1910,p.234, a new family for Kirkbya and Amphissites.

KIRKBYIDAE of Henningsmoen 1953,p.197,272 includes Amphissitinae of Cooper 1941, and the following genera: Kirkbya, Amphissites, Albanella, Aurikirkbya, Ectodemites, Polvtylites, Knichtina, Arcyzona, Roundyella, Kellettina, Franklirclla, Edita, Brillius, Savasella, and many genera he considers synonyms of these genera.

KIRKBYINA [Beyrichiella (?) reticosa. Jones and Kirkby 1886b,p.260,pl.8,f.15,16] Ulrich and Bassler $1908, \mathrm{p} .322$ [Kloedencllidae by Bassler and Kellett 1934,p.30; Kloedenellidae-Kloedenellinae by Henningsmoen 1953,p.243,271] Carboniferous, Scotland.

KIRKBYTTES [K. upsoni] Johnson 1936,p.585 [Kirkbyidae hy Johnson, also Agnew 1942,p.759] Pennsylvanian of Nebraska. 
KIIEANA [K. alata] Nartin 1940,p.322-323 [Cytheridae-Cytherideinae by Martin] Jurassic, Germany.

KLIELLA [K. hyaloderma] Schäfer 1945,p.857 [Cytheridae-Kliellinae by Schäfer] Subterranean, Greece.

KLIELIINAE Schäfer 1945,p.857, subfamily of Cytheridae for Kliella and Nannokliella, r.gen.

KIOEDENELLA [KIoedenia pennsylvanica Jones 1889a,p.341] Ulrich and Bassler 1908,p.317 [Kloedenellidae by Bassler and Kellett 1934,p.30; Kloedenellidac-Kloedenellinae by Henningsmoen 1953,p.242,270] Devonian, Pennsylvania.

KLOEDENELIIDAE Ulrich and Bassler 1923,p.312, a new family to include Eukloedenella, Kloedenella, Dizygopleura, Jonesina, Kirkbyina, Beyrichiella, Beyrichiopsis.

KLCEDENELIINA [K. heimdalli] Coryell and Johnson 1939,p.216 [Kloedenellidae by CEJ; Kloedenellidae-Kloedenellinae by Henningsmoen 1953,p.242,270] Mississippian of Illinois.

KLOEDENELIINAE Ulrich and Bassler 1908,p.317, a subfamily of Beyrichiidae for Kloedenella, Beyrichiopsis, Beyrichiella, Jonesina, Kirkbyina, and ?Kyammodes. 
KLOEDENELIINAE Henningsmoen 1953,p.242,270, a subfamily of Kloedenellidae for Kloedenella, Eukloedenella, Dizygopleura, Poloniella, Jonesina, Oliganisus, Gillina, Kloedenellina, Neokloedenellina,

Lochriella, Sansabella, Coelonella, Microcoelonella, Hastifaba, Geisina, Knoxina, Sargentina, Perprimitia, Carboprimitia, Kirkbyina, Microparaparchites, Paraparchites, Proparaparchites, Pseudoparaparchites, Geffonina, Geffenites, Kellettella, Chesterella, Beyrichiella, Kirkbyella, Balantoides, Bounsella, Cornigella, ?Bicornella, ?Beyrichiana, ?Aechminella, and $\mathcal{E}$ synonyms of these genera.

KIOEDENTA [Beyrichia wilckensiana Jones 1855,p.89,pl.5,f.17, 18] Jones and Holl $1886 \mathrm{a}, \mathrm{p} .362$ [Leperditiidae-Beyrichiinae by Jones 1901,p.147; Zygobolbidae-Kloedeninae by Bassler and Kellett 1934,p.360; Beyrichidae-Beyrichilnae by Henningsmoen 1953, p.236,238,269] Silurian drift, Germany.

KIOEDENINAE Ulrich and Bassler 1923,p.306, new subfam. of Zygobolbidae, for Plethobolbina, Mastigobolbina, Kloedenia, Welleria, Kyammodes, Zygobeyrichia, Steusloffia. [ = Beyrichiinae by Henningsmoen $1953, p \cdot 236]$. 
KNTGHTINA [Amphissites allerismoides Knight 1928,p.265, pl.32,f.10a-e; pl.34,f.4] Kellett 1933,p.97 [Kirkbyidae by Kellett] Pennsylvanian of Missouri.

KNOXIELLA [K. semilukiana] Egerov, 1950,p.90,fide Polenova 1952,p.94 [Kloedenellidae-Knoxinae by Polenova] Devonian to Carboniferous by Polenova.

KNOXINA [K. lecta] Coryell and Rogatz 1932,p.383 [Kloedenellidae by CER; Kloedenellidae-Kloedenelinnae by Henningsmoen 1953,p.271] Permian of Texas.

KNOXINAE Egerov 1950,p.?, by Polenova 1952,p.94, a subfamily of Kloedenellidae.

KNOXITES Egerov 1950,p.84, fide Polenova 1952,p.94.

KRAUSELLA [K. inaequalis] Ulrich 1894,p.691 [Beecherellidae by Ulrich; Cypridae-Cyprinae-Paracyprides by Van den Bold 1946,p.22] Ordovician, Illinois.

KRITHE [Cythere (Cytherideis) bartonensis Jones 1857, p.50, pl.5,f.2,3] Brady, Crosskey and Robertson 1874, p.183, new name for Ilyobates Sars 1866, not Kraatz 1858 [Cytheridae-?Cytherideinae by Sars 1925,p.164-165] Recent, North Atlantic.

KYAMMODES [K. whidbornei] Jones 1888b,p.295 [Barychilinidae by Ulrich 1894,p.632; Zygobolbidae-Kloedeninae by Bassler and Kellett 1934,p.370] Devonian, England. 
KYPHOCYTHERE [Cythereis Iimicola Norman $1865, p .20, p 1.6, f .1-4$ ] Sars 1925,p.180-181 $[=$ Palmenella Hirschmann 1916, p. $58 \mathrm{C}$, which has same genotype.

I

LACCOCHILINA [Eurychilina estonula Opik 1935,p.9,fig.]

Hessland 1949,p.258 [Hollinidae-Eurychilininae by Hessland] Ordovician, Esthonia.

LACCOPRIMITIA [Primitia centralis Ulrich 1890, p.130,pl.10, f.1,2a-c] Ulrich and Bassler 1923,p.299 [Primitidiae-Primitilnae by Bassler and Kellett 1934, p.17; Eurychilinidae-? Euprimitiinae by Henningsmoen 1953, p.229,268] Ordovician, Ohio.

LADDELLA [L.insueta] Spivey 1939,p.175 [Family not indicated by Spivey; Incertae sedis, probably not an ostracod by Aonew 1942, .759 ; Beyrichiacea of uncertain family by Henningsmoen 1953,p.274] Ordovician, Iowa.

LAMARELLA [L. thurmani] Corneis and Funkhouser 1939,p.336 I = Sansabe11a by Cooper 1941,p.60, and by Agnew 1942,p.759] Mississippian of Illinois.

IANGTONIA [L. setina] Anderson 1939,p.304 [Cypridae-Rostocyprinae by Anderson; cypridina (Pseudocypridina) by Sylvester-Bradley 1949,p.126-127 = Pseudocypridina by Peck 1941,p.318,319] Jurassic, England. 
LEGUMINOCYTHEREIS [I. scarabaeus] Howe, in Howe and Law 1936, p.61 [Cytheridac by Howe] Oligocene, Mississippi.

LEIGHTONELLA [L. torta] Croneis and Gale 1939,p.263 [?Aechminidae by CEG; = Deloia by Cooper 1941,p.54, and by Agnew 1942,p.759] Mississippian of Illinois.

LEIOPRIMITIA [L. punctata] Kummerow 1939,p.17[Primitiidae-Primitinae by Kummerow] Lower Carboniferous, Germany •

IEIODITIA (UIrich,Nss.) Jones 1891,p.94 = Elpe by Bassler and Kellett 1934,p.297,372 = Elpezoe by Přibyl 1951, p.121.

LENIOCYTHERE [I. lcbanonensis] Howe 1951b,p.22 [Cytheridae-Cytherinae by Howe] Eocene, Florida.

LEPERDITACEA Bassler and Kellett 1934,p.13-16, a Superfarily to include the families Leperditiidae and Leperditellidae.

IEPERDITELLA [Leperditia inflata Ulrich 1892,p.265,pl.9, f.12-15] Ulrich 1894,p.636 [Leperditellidae by Ulrich and Bassler 1906,p.149; Aparchitidae by Ulrich and Bassler 1923,p.296; Leperditellidae by Bassler and Kellett 1934,p.372; Aparchitidae by Schmidt 1941,p.17,18,71; ?Leperditellidae-Leperditellinae by Henningsmoen 1953,p.232,250,251,275] Ordovician, Kentucky. 
LEPERDITELLIDAE Ulrich and Bassler 1906,p.149, a family for the new genus paraparchites, also Leperditella and the closely related Aparchites. They consider Cytherella was derived from Paraparchites.

LEPERDITELLINAE Henningsmoen 1953,p.250,275, a subfamily of Leperditellidae for the single genus Leperditella.

LEPERDITIA [I. britannica] Rouault 1851,p.377 [Leperditidae by Jones 1856b,p.84; Leperditiidae by Jones 1870a, p.187; Leperditiidae-Leperditiinae by Swartz 1949, p.311-312] Devonian, France.

LEPERDITIACEA Henningsmoen 1953,p.249, a Superfamily to include the family Leperditiidae and the family Leperditellidae with the ?subfamilies Leperditellinae, Conchoprimitiinae, and Eridoconchinae.

IEPERDITIADAE Jones $1870 \mathrm{a}, \mathrm{p} .187$, as used this family includes the genera: Leperditia, Isochilina, Primitia, Beyrichia, Kirkbya, and Moorea. [It is a change in spelling from Leperditidae Jones 1856.]

LEPERDITIDAE Jones 1855b,p.84, a family of Phyllopoda? for the genus Leperditia.

LEPERDITIIDA Pokorny 1953,p.222,227,231, An Order of the Class Ostracoda for the forms placed in the family Leperditiidae Jones 1856. 
LEPERDITIIDAE of Ulrich 1894,p.632 includes Leperditia, Leperditella, Isochilina, Aparchites, Schmidtella, and ? Aechmina.

LEPERDITIINAE Swartz 1949,p.311, a subfamily of Leperditiidae to include: Leperditia, Hermannina, Eoleperditia, Chevroleperditia, Briartina, Holtedahlites, Paenaequina.

LEPIDILLA [L. anomala] Matthew 1886,p.62 [Removed from ostracoda to Conchostraca by Ulrich and Bassler 1931,p.99] Cambrian, New Brunswick.

LEPIDITTA [L. alata] Natthew 1886,p.61. [Removed from ostracoda to Conchostraca by Ulrich and Bassler 1931,p.93] Cambrian, New Brunswick.

LEPTOCYPRIS [L. papyracea] Sars 1903,p.28 [Not Leptocypris Boulenger $1900=$ Astenocypris G. W. Müller, 1912, p.203, new name] Raised from dried Sumatra mud.

LEPTOCYTHERA Sars 1925, p.171, typographical error for Leptocythere.

LEPTOCYTHERE [Cythere pellucida Baird 1850,p.178,pl.21,f.7] Sars 1925,p.XI, 171-177 [Misspelled Leptocythera by Sars in title, but obvious typographical error, as Leptocythere in all other places in volume and in description of species. Cytheridae-Cytherinae by Sars] Recent, North Atlantic. 
IEPTOCYTHERIDEA [Cytheridea (Leptocytheridea) fragillissima] Stephenson 1937,p.156 [As a suhgenus of Cytheridea by Stephenson; = juvenile Haplocytheridea by Stephenson 194l,p.425] Miocene of Mississippi.

IEPTOPRIMITIA [L. compressa] Kummerow 1953,p.3] [Primitiidae by Kummerow] Devonian, Poland.

LEUCOCYTHERE [I. mirabilis] Kaufmann 1892,p.394 [Cytheridae-Limnocytherinae] Recent, Switzerland.

LICHWINIA Posner 1951?,p.?, compared to Evlanella by Polenova $1952, p \cdot 90$.

IICHWININAE Posner 1951?,p.?, a subfamily of Kloedenellidae, in which Polenova 1952,p.89,places Evlanella.

LIMBATULA [L. symmetrica Zaspelova ] Zaspelova 1952,p.190 Drepanellidae by Zaspelova] Devonian, USSR.

LIMNICYTHERE Brady $1868 \mathrm{~b}, \mathrm{p} .418$, a change in spelling = Limnocythere Brady 1868a.

LIMNICYTHERIDEA Forel 1894,p.602, nom.nud., fide G. W. Müller $1912, p \cdot 328$

LIMNICYTHERINAE Sars 1925,p.148,149, a subfamily of Cytheridae for Limnicythere, Leucocythere, and Gomphocythere. 
LIMNOCYTHERE [Cythere inopinata Baird 1843,p.195, figs.a-c, designated by Brady and Norman 1889,p.170] Brady $1868 \mathrm{a}, \mathrm{p} .121$ [Cytheridae-Limnicytherinae by Sars 1925,P.148; Cytheridae-Limnocytherinac by Klie $1938 \mathrm{a}, \mathrm{p} .150]$ Recent, Europe.

IIMNOPRIMITIA [Cypris arcuata Bean 1836,p.377,fig.55] Kummerow 1949,p.48 Carboniferous, England. [Redescribed as new by Kummerow 1953,p.15].

LINDSAYELLA [L. rugosa] Coryell and Williamson 1936,p.1. [Primitiidae by CGW; Drepanellidae-Drepanellinae by Schmidt 1941,p.50; Drepancllidae-?Aechmininae by Henningsmoen 1953,p.269] Silurian, Indiana.

LINEOCYPRIS [I. trapezoidea] Zalányi 1929,p.40 [Cypridae-Candoninae by Zalányi] Neogene, Servia.

LII!OCHELCS [L. vagans] Brady 1907b,p.7 [Cytheridae near Xestoleberis by Nïller 1912,p.304] Recent, Antarctic.

LIOCYPRIS [I.. grandis] Sars 1924a,p.114 [Cypridae nearest Homocypris by Sars] Recent, South Africa.

LOCHRIEIIA Scott 1940,p.1977, abst. nom.nud.

LOCHRIEI.I.A [L. ottcrensis] Scott 1942,p.155 [Kloedenel1idac by Scott; Kloedenellidae-Kloedenellinae by Henningsmoen 1953,p.242,270] Mississippian of Montana.] 
LOKIUS [L. sigynae] Coryell and Johnson 1939,p.216 [= Perprimitia Croneis and Gale by Cooper 1941, p.58, and by Agnew 1942,p.759; however is recognized by Scott 1942,p.159, who places the genus in the Kloedenellidae, instead of Acronotellidae as CEJ had done.] Mississippian of Illinois.

LOONEYELLA [Cythere monticula Jones 1893a,p.38i,pl.15, f.13a, b.] Peck 1951b,p.575 [Cytheridae near Hutsonia by Peck] Cretaceous, Wyoming.

LOPHOCYTHERE [Cytheridea ostreata Jones and Sherborn 1888,p. 271,pl.4,f.6] Sylvester-Bradley 1948b,p.194 [Cytheridae-Progonocytherinae by S-B] Jurassic, England.

LOXOCONCHA [Cythere rhomboidea Fischer 1855,p.656] Sars 1866,p.61. [Cytheridae-Loxochonchinae by Sars 1926,p.217] Recent North Atlantic. [Brady and Norman 1889,p.183 designated Cythere impressa Baird 1850 as the genotype, placing $\underline{C}$. rhomboidea in its synonymy. G. W. Müller discovered $\underline{\text {. }}$ impressa Baird was proceded by $\underline{C}$. impressa $M^{2} \mathrm{Coy}$ 1844 and renamed it C. bairdii, which was invalid C. rhomboidea was the first valid name for the species.] 
LOXOCHONCHINAE Sars 1925,p.217, a subfamily of Cytheridae for Loxochoncha and Cytheropteron.

LCXOCYTHERE [I. Crassa] Hornibrook 1952,p.30 [Cytheridae-Cytherinae] by Hornibrook] Recent, New Zeeland.

LOXOLEBERIS [Lapsus pennae for Loxoconcha longipes] Sars $1866, \mathrm{p} .130$.

LUCASELIA [I. mundula] Stewart 1936,p.76] [Bairdiidae by Stewart; Cytherellidae by Agnew] Devonian, Ohio.

LUIULARIA, Discussed by Polenova 1952,p.82 under ?Acronotellidae-Graviinae.

LUVULA [L. palmerae] Corycll and Fields 1937,p.13 [Cytheridac-Bythocytherinae by CEF] Miocene, Panama.

MACHAERINA [Bythocythere tenuissima Norman 1869,p.294] Brady and Norman 1889,p.237, new name for Xiphichilus Brady 1870. preoccupied by Xiphocheilus Bleeker 1856 [Paradoxostomatidae by BEN; Cytheridae-Cytherinae by G. W. Müller,1894,p.308] Recent, British Isles.

MACROCONCHOECIA [Conchoecia reticulata Nililer 1906a,p.64, pl.12,f.10-17 and C. caudata Milller 1890, p.276, pl.29, f.45-49, with the first apparently intended 
as the genotype] Granata and Caporiacco 1949, p.13,15,33 [Halocypridae by GEC] Recent, Atlantic.

ACROCYPRIA [Nacrocypris sarsi G. W. Müllor 1912,p.123, new name for Bairdia angusta Sars $1866, p .22$, not Cythere (Bairdia) angusta (Münster) Jones 1849, p.26] Sars 1923,p.60. [Cypridae-Macrocyprinae by Sars] Recent, North Atlantic.

YACROCYPRIDAE Alm 1915,p.28, a Gruppe of the Cypridae, [which he uses as a superfamily] with subfamily Macrocyprinae, for the genus Macrocypris.

IAACROCYPRIDAE Sylvester-Bradley $1948 \mathrm{a}, \mathrm{p} .67$, a family which he placed with the Bairdiidae in the superfamily Bairdiacea, but with the single genus Nacrocypris. NACROCYPRIDITA [Cypridina castanea Brady 1897,p.88,pl.16, f.1-4] Skogsberg 1920,p.280 [as subgenus of Cypridina by Skogsberg] Recent, North Atlantic. NACROCYPRINAE G. W. Mül] er 1912,p.120, a subfamily of Cypridae for the genus Macrocypris.

MACROCYPRIYAE AIm 1915,p.2E, a subfamily of the Gruppe Macrocypridae, for the genus Macrocypris.

NACROCYPRIS [Cythere minna Baird 1850,p.171,pl.20,f.4a-d] Brady 1858a,p.119 [Cypridae by Brady; Bairdiidae by Brady and lioman 1889,p.112; Cypridae-- 
Macrocyprinae by G. W. Muller 1912,p.120;

Cypridae-Macrocypridae-Macrocyprinae by Alm 1915, p.28; Bairdiacea-Macrocypridae by Sylvester-Bradley 1948a,p.67] Recent, British Isles.

MACROCYPROIDES [M. clemontensis] Spivey 1939,p.174

[Bairdiidae by Spivey] Ordovician, Iowa.

MACROCYTHERE [Cythere simplex Norman $1865, \mathrm{p} .17, \mathrm{pl} .5, f .1-4$ ] Sars 1926,p.240 [Cytheridae-Bythocytherinae by Sars] Recent North Atlantic. $[=$ Jonesia Brady $1866 \mathrm{a}, \mathrm{p} .362$ which has Cythere simplex Norman as genotype. Norman's species was preceded, however, by Cythere auriculata simplex Cornuel. 1645,p.213, pl.3, f.10,11].

NACROCYTHERINA [N. gaturensis] Coryell and Ficlds 1937, p.I6 [Cytheridae-Bythocytherinae by CEF] Miocene of Panama.

MACRODENTINA [N. Iineata] Niartin 194C, p.325 [Cytheridae-Cytherinae by Martin] Jurassic, Germany.

NACRONOTELLA [Mi. scofieIdi] Ulrich 1894,p.683 [Beyrichiidae by UIrich; Kirkbyidae by UIrich and Bassler 1923, p.315; Aparchitidae by Kay 1940a,p.244 and by Henningsmoen 1953,p.231,268] Ordovician, Minnesota. 
MAMMOIDES [M. mammillata] Bradfield 1935,p.36, Pennsylvanian, Oklahoma $[=$ Aechminella Harlton by Kellett 1936 , p.771, and by Agnew 1942,p.759].

MANAWA [M. tryphena] Hornibrook 1949,p.470 [Punciidae by Hornibrook] Recent off New Zealand.

NARATIA [M. mara] Kay 1940a,p.258 [Primitiidae-Bollinae by Kay; Drepanellidae-?Bolliinae by Henningsmoen 1953, p.233,268] Ordovician, Ontario.

MARGINIA [M. sculpta] Polenova 1352,p.96 [Kloedenellidae-Knoxinae by Polenova] Devonian, USSR.

N:ASTIGOBOLBINA [M. typus] Ulrich and Bassler 1923a,p.3c7 [Zygobolbidae-Kloedeninae by Bassler and Kellett $1034, p \cdot 4 \mathrm{CB} ;$ Deyrichidae-Zygobolbinae by Fonningsmoun 1953,p.238,259] Silurian, West Virginia.

NAURYELIA [ $\because$. mammillata] Ulrich aid Bassler 1923a,p.316 [Kirkbyidae by UEB; Beyrichiacea of uncertain family by Henningsmoen 1953,p.274] Mississippian, Tennessec.

MEGALOCYPRIS [M. princeps] Sars 1898,p.4 [Cypridae-Cyprinae by Müller 1912,p.219] Recent, South Africa.

MELANELIA [M. hemidiscus] Wade 1911,p.451 [not Melanella Bory 1824, Palyg.] $=$ Vosdesella Baker 1924,p.I87, new name? Ordovician, Wales. [ Jonesella Ulrich by Bassler and Kellett $1934, \mathrm{p} \cdot 343$. 
MENOEIDINA [M. subreniformis] Stewart 1936,p.752 [Cytherellidae by Stewart] Devonian, Ohio.

MESOCYPRIS [M. pubescens] Daday 1908,p.374 (described arain by Daday 1910,p.197) [Cypridae near Cypris by Daday] Recent, German East Africa.

MESOGLYPHA [M. rediocre] Cooper 1914,p.44 [Glyptopleuridae by Cooper; Kloedenellidae-Glyptopleurinae by Henningsmoen 1953,p.245,272] Mississippian of Illinois.

MESOMPHALUS [M. hartleyi) Ulrich and Bassler 1913a,p.522 [Zygobolbidae-Drepanellinae hy UGB; Beyrichiidae-Bryrichiinae by Henningsmoen 1953,p.232,236,238. 269] Devonian of Maryland and West Virginia.

METACANDONA [M. bispinosa, $\because$. werestschagini] Bronstein 1930, p.136 i = Pseurocaidona Kaufmann by Bronstein 1932, p.333, and by Bronstein 1947,p.188] Recent, Lake Baikal.

M.LTACO:CHOECIA iGenotype not stated but enus set up for $\underline{M}$.

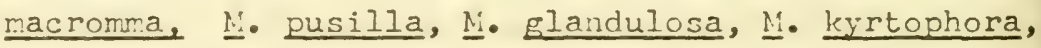
‥ nasotuberculata, $M$. rotundata, $\mathrm{N}$. isocheira, all of which were originally described by G. W. Müller as Conchoecia. Of these $M$. rotundata is discussed in detail and may be considered as their genotype] Granata and Caporiacco ]949,p.12,15,22. [Halocypridae by CEG] Recent, Atlantic. 
NETACYPRIS [ $\mathrm{N}$. cordata] Brady and Robertson 1870,p.19 [Cytheridae-Xestoleberinae by Sars 1928,p.248; Kloedenellidae, descended from Knoxina, Geisina, or Jansabella by Kellett 1943,p.626; Cytheridae-Xestolcbcrinac by Nartin 1940,p.335] Recent, Europe. 1:EZON:PHALUS Zaspelova 1952,p.158,159, = Nesomphalus Ulrich and Bassler 1913, a change of spelling.

YICA [N. inaequalis Netjkaia,n.sp.] Netjkaia 1952,p.22E [Incertae sedis by Netjkaia] Ordovician, USSR. [Not Mica Budde-Lund 1908,p.281]

NICROCHEILINELLA [Nicrocheilus distortus Geis 1932,p.182, pl.25,f.15a,b.] Geis 1933,p.Il2, [new name for Microcheilus Geis, 1932, Not Kittl 1894.

Bairdildae by Geis; Cytherellidae by Bassler and Kellett 1934,p.42] Mississippian of Indiana.

MICROCHEIIUS Geis 1932,p.181,182, not KittI $1894=$ Microcheilinella Geis 1933,p.112, new name.

MICROCOELONELLA [N. scanta] Coryell and Schn I939,p.597 [Leperditellidae by CES; KIosdenellidac- KIoedenelliaac by Fienningsmoen 1953,p.271] Mississippian of West Virginia. 


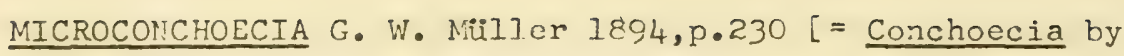
Miiller]; G. W. Müller 1912,p.482; Brady 1897, p.97; Granata and Caporiacco 1949,p.12 and many others $=$ Mikroconchoecia Claus 1891 .

MICROCYPRIS [M. reptans] Kaufmann 1900c,p.132 [ = Eucypris by G. W. Müller 1912,p.173] Recent Switzerland.

MICROCYTHERE [9 species, the first $M$. inflexa being designated genotype by Var den Bold 1946,p.26] G. W. Nüiller 1894;p.327 [Cytheridae-Paradoxostominae by Nüller; Cytheridae-Microcytherinae by KIie 1938a,p.215] Recent, Gulf of Naples.

MICROCYTHERINAE Klie 1938a,p.215, a subfamily of Cytheridae for the single genus Microcythere.

MICROCYTHERURA [M. migrescens] G. W. Müller 1894,p.383

[Cytheridae-Xestoleberinae] Recent, Gulf of Naples.

NICROPARAPARCHITES [M!. spinosus] Croneis and Gale 1939, p.256 [Leperditellidae by CEG; Kloedenellidae-Kloedenellinae by Henningsmoen 1953,p.243-271] Mississippian of Illinois.

MICROXESTOLEBERIS [M.nana] G. W. Müller I894,p.339 [Cytheridae-Xestoleberinae by Sars 1928, p.242] Recent, Gulf of Naples. 
MIKROCONCHOECIA [Halocypris clausi Sars 1888,p.259,pl.11, f.7-10;p1.14,f.6-18] Claus [1890] 1891,p.22; 1891a,p.73,pl.20 [Halocypridae-Conchoecinae by Claus; = Conchoecia by G. W. Müller 19l2,p.59; Halocypridae by Granata and Caporiacco 1949,p.14, 18] Recent, Mediterranean.

MILLERATIA [Beyrichia cincinnatiensis Miller 1875,p.350, fig.25] Swartz 1936,P.567 [Primitildae by Swartz; Primitidae-?Primitinae by Schmidt 1941,p.25; ?Leperditellidae-?Eridoconchinae by Henningsmoen 1953,p.256,275] Upper Ordovician of Ohio.

VIITONELIA [Y. shupei] Sohn 1950,p.37 [Miltonellidae by Sohn] Permian of Texas.

MIITONEIIIDAE Sohn $1950, \mathrm{p} .37$, a family crected for the genus Miltonella; tentatively assigned to the Beyrichiacea by Henningsmoen 1953,p.273 because of its straight back.

MIRACYTHERE [M. novaspecta] Hornibrook 1952,p.61 [Cytheridae-Bythocytherinae by Hornibrook] Recent, New Zealand.

MIROCHILINA [N. jarovensis] Bouček 1936,p.51 [Primitiidae-Eurychilininae by Boučk; Beyrichiacea of uncertain family by Henningsmoen 1953,p.249,274] Silurian, Bohemia. 
MONOCERATELLA [M. teres] Teichert 1937a,p.114 [Acronotellidac by Teichert] Ordovician, Arctic Canada.

MONOCERATINA [N. ventrale] Roth 19,28,p.15-19 [Primitiidae by Roth; Beyrichiidae by Harlton 1933,p.21;

Cytheridae by Alexander 1934,p.60; Acronotellidae

by Swartz $1936, p \cdot 555,560 ;$ C by Van den Bold 1946,p.34] Pennsylvanian, Oklahoma.

MONOCULUS [ 9 species: M. polyphemus, foliaceus, apus,pulex, pediculus, quadricornis, conchaceus, lenticularis, telemus] Linnaeus, $1758, p .631,635$ [of the precedins species, $\underline{M}$ - conchaceus has been considered to be an ostracod, principally by Jurine 1820, who referred his fresh-water ostracods to this genus. G. W. Muller 1912,p.222 considered both the genus and species uncertain.]

MONOPIA [Cypridina monopia Claus $1873, p \cdot 222, f \cdot 21-31=$ Monopia flaveola Claus 1873,p.227,f.21-31] Claus 18.73, p.225 [ = Eumonopia Claus 1891b,p.234; = Cypridina by G. W. Muller 1912,p.13].

MOOREA [M. silurica] Jones and Kirkby M.S., Jones and Holl $1869, p .225,226$ [Not "Moorea tenuis Jones,M.S." Noore 1867,p.494,559; "Moorea obtusa Jones" Noore 1867,p.523; nor Moorea obesa Jones N"S" Noore 1867, p.525,559, as these are nude names. Beyrichiidae by Ulrich 1894,p.632; Youngiellidae by Bassler and Kellett 1934, p.35] Silurian, England. 
MOOREINA [M. johnsvalleyensis] Harlton 1933, p.21 [Beyririchildae by Harlton; Primitiidae by Bassler and Kellett 1934,p.414; Acronotellidae by Swartz 1936, p.555; = Paracytheridea G. W. Müller by Van den Bold 1946, p.26; Beyrichiacea of uncertain family by Henningsmoen 1953, p.274] Pennsylvanian, Oklahoma.

MOORITES [Moorites hewetti Coryell and Billings 1932, p. $183, \mathrm{pl} .18, f .5=$ Moorites minutus $($ Warthin $)=$ Glyptopleurina? minuta Warthin $1930, \mathrm{p} .67, \mathrm{pl} .5$, f.6, by Kellett 1933,p.104] Coryell and Billings 1932,p.182 [Kirkbyidae by CGB; Youngiellidae by Kellett 1933,p.103; ?Youngiellidae, but may be related to Cytherelloidea by Henningsmoen 1953, p.246,273] Pennsylvanian, Oklahoma.

MORININA [M. dorsispinata] Anderson 1939,p.302 [Cypridae-Rostocyprinae by Anderson; = Cypridea by Martin $1940, p .281 ;=$ subgenus of Cypridea by Sylvester-Bradley 1949,p.127] Jurassic, England.

MORRISONIA [M. wyomingensis] Branson 1935,p.521 [Not Morrisonia Grote 1874; = Theriosynoecum Branson 1936,p.323, new name] Morrison formation, Jurassic? Wyoming. 
MORROWINA [Cytherella excavata Alexander 1934b, p.211-212, pl.32,f.3,4; pl.35,f.5,6\} Loetterle 1937,p.5l. [Cytherellidae by Loetterle] Midway Eocene of Texas.

MUTILUS Neviani 1928,p.72,90, a "Gruppo" = subgenus or perhaps genus of "Cythereis", and based on 3 species: Cythere macropora Bosquet 1852,p.97,pl.5, f.2; Cythereis (Mutilus) excancellata Neviani, new name for Cythere cancellata Lienenklaus 1894 , p.204,pl.14,f.5; and Cythereis Mutilus lacticancellata Neviani 1928,p.93,pl.2, f.66-68. Bosquet's species from Eocene of France, though Neviani indicated all from Pliocene of Pisa, Italy.

MYODOCOPA Sars 1866, p.99, a Suborder consisting of the families Cypridinadae and Conchoeciadae.

MYODOCOPIDA Pokorny 1953,p.222,227,231, an Order of the Class Ostracoda with a taxonomic range similar to the group G. W. Müller designated as tribus Myodocopa. It comprises the suborders Myodocopa G. O. Sars 1866, and Cladocopa Sars 1866. "According to Triebel, 1941, it is possible that the Paleozoic family Entomozoidae (Jones, 1873) Pribyl, 1949, also belongs here." 
NANNOCANDONA [N. faba] Ekman 1914,p.19 [Cypridae-Candoninae by Ekman] Recent, Sweden.

NANNOKLIELIA [N. dictyoconcha] Schäfer 1945,p.860 [Cytheridae-Kliellinae by Schäfer] Subterranean, Greece.

NANOPSIS [Beyrichia nanella Moberg and Segerberg 1906,p.76, pl.3,f.27-28] Henningsmoen 1954a,p.54 [incertae sedis by Henningsmoen] Ordovician, Norway.

NAVECYTHERE [N, delicata] Coryell and Fields 1937,p.7

[Cytheridae-Cytherideinae by CEF; = Archicythereis by Van den Bold 1946,p.29; = juvenile Cativella]

NEHDENTOMIS [Entomis (Nehdentomis) nehdensis] Matern 1929, p.54 [subgenus of Entomis by Matern; subgenus of Entomozoe: - Entomozoacea-Entomozoidae-Entomozoinae by Pribyl 1951,p.105] Devonian, Germany.

NEMATOHAMMA [N. obliqua] Brady and Norman 1896,p.680

[Sarsiellidae by BEN; = Sarsiella by G. W. Müller 1912, p.38] Recent off Ireland.

NEOAPARCHITES [Primitia obsoleta Jones and Holl 1865,p.423, pl.13,f.12a-c] Bouček 1936,p.39 [Aparchitidae, and as subgenus of Aparchites by Bouček; Primitiopsidae by Teichert 1937b,p.45, where Neoaparchites is raised to generic rank and discussed at length; ?Aparchitidae by Henningsmoen 1953,p.231,268] 
NEOBEYRICHIOPSIS [N. emporiensis] Tasch 1953,p.401 [closest aff. to Beyrichiopsis, by Tasch] Pennsylvanian, Kansas.

NEOCHILINA [N. binsenbachensis] Matern 1929,p.33 [Primitiidae-. Eurychilininae by Matern; Hollinidae by Swartz 1936, p. 551; Beyrichiacea of uncertain family by Henningsmoen 1953,p.228, 229, 274] Devonian, Germany.

NEOCYPRIDOPSIS [N. debilis] Klie 1940, p.61 [Cypridae-Cyprinae by Klie] Recent, Brazil.

NEOCYPRIS [6 species: N. gladiator, mutica, variegata, obtusata, elIiptica, mucronata] Sars 1901, p.29 I in part $=$ Acanthocypris, part Cypris by Sharpe $1903, \mathrm{p} .983 ;=$ Eucypris at least in part by Daday $1905, \mathrm{p} .243 ;=$ Strandesia by G. W. Müller 1912, p.186] Raised from dried South American mud.

NEODREPANELLA [N. tricornis, Batalina, 1941] Zaspelova 1952,p.163 [Drepane]1idae-Neodrepanellinae by Zaspelova] Devonian, USSR.

NHODREPANELLINAE Zaspelova 1952,p.162, subfamily of Drepanellidae, for Neodrepanella, Bicornella, Tetracornel1a, Limbatula. 
NEOCYTHERIDEIS [N. elongatus] Puri 1952,p.912 [Cytheridae-Cytherideisinae by Puri] Recent, England.

NEOKLOEDENELLA [N, prima] Croneis and Funkhouser 1939,p.341

[Kloedenellidae by CEF; Kloedenellidae-Kloedenellinae by Henningsmoen 1953, p.242, and spelled Neokloedenellina,p.270] Mississippian of Illinois.

NEOKLOEDENEILINA Henningsmoen 1953,p.270, a typographical error $=$ Neokloedenella.

NEOLIMNOCYTHERE [N, hexaceros] Delachaux 1928,p.59

["Cytheridees by Delachaux] Recent, Peru.

NEOMONOCERATINA [N, columbiformis] Kingma 1948,p.94 [Cytheridae-Bythocytherinae by Kingma; = a subgenus of Paijenborchella Kingma, by Ruggieri 1953,p.1-7] Upper Miocene Sumatra and living Java Sea.

NEPHOKIRKOS [N. aquaplanus] Howe 1951b,p.11 [Cytheridae-Cytherinae by Howe] Eocene, Florida.

NESIDEA [N, hirta] Costa 1849,p.183-187 [Nesideidae by G.W. Müller 1912,p.241; = Bairdia M'Coy, 1844 by Sars 1923,p.66] Recent, Gulf of Naples.

NESIDEIDAE G. W. Mïller 1912,p.241, based on the genus Nesidea, Bythocypris, and Anchistrocheles. 
NEWNHAMIA [N. fenestrata] King 1855, p.67 [Cypridae-Notodromadinae by Sharpe 1903,p.974; Cypridae-Cyprinae by G. W. Müller 1912,P.158; Cypridae-w Notodromadinae by Henry 1923,p.270] Recent, Australia.

NEWSOMITES [N. pertumidus] Morris and HiI1 1952,p.14 [Bairdidae by MEH] Silurian, Tennessee.

NODEilA [N. svinordensis Zaspelova,n.sp.] Zaspelova 1952, p.187 [Drepanellidae-Nodellinae by Zaspelova] Devonian, USSR.

NOFELI. INAE Zaspelova 1952,p.386, a subfamily of Drepanellidae for Nodella, Pseudonodella, Subtella, Schweyerina, and Acanthodella.

NORMANIA Erady, in Jones 1865, p.306 (in a 1ist, not described); name abandoned by Brady 1865, p.189, in footnot. NORMAN IA Brady $1866 a, P .382$ [6 species: N. avs lana, affinis, glabra, grisea, modesta, dorso-tuberoulata. I Proceded by Loxoconcha Sars [1865] 1866; Is Loxoconcha by Brady $1868 \mathrm{~b}, \mathrm{p} .432$.

NoTHozot, [N. peilens Barrande in Bigsby 1868,p.200, 1ist] Barrande $1872, \mathrm{p} .536, \mathrm{pl} \cdot 23,27$.

NOTODHMADINAL Kaufmann 1900a,p.104,105; 1900b,p.251, a subfamily of Gyprididae for Notodromas and Cyprois. 
NOTODROMAS [Cypris monacha O.F.Müller $1785, p .60, p l .5, f .6$ ] Liljeborg 1853,p.91 [Cyprididae-Notodromadinae by Kaufmann 1900a,p.104; Cypridae-Cyprinae-Notodromides by Sars 1925,p.99; Cypridae-Cyprinae-Notodromini by Bronstein 1947,p.97-99] Recent, Europe.

NOTODROMIDES Sars 1925,p.99, a Group of Cypridae-Cyprinae for the genera Notodromas, Cyprois, Newnhamia, and Hungarocypris.

NOTODROMINAE Hoff $1942 a, p .147$, a change in spelling of Notodromadinae, Kaufmann. Hoff includes Notodromas and Cyprois.

NOTODROMINI Bronstein 1947,p.97-99, a Tribe of CypridaeCyprinae, for the genera Notodromas and Cyorois. NOVAKINA [N. applanans] Boudek 1936,p.56 [Primitiidae-Aechmininae by Bouček; Beyrichiacea of uncertain family by Henningsmoen 1953,p.249,274] Silurian, Bohemia.

NUFERELLA [N. infrequens] Bradfield 1935,p.45 [Kloedenellidae by Bradfield; : Jonesina by Cooper 1946,p.113] Pennsylvanian, Oklahoma.

0

OCCULTOCYTHEREIS [O. delumbata] Howe 1951,p.19 [Cytheridae-Cytherinae by Howe] Eocene, Florida. 
OCTONARIA [0. octoformis] Jones 1887b,p.404 [ThIipsuridae by Ulrich 1894,p.632] Silurian, England.

OCTONARIELLA [0. typa] Bassier 1941,p.27 [Thlipsuridae by Bassler; = Strepulites by Warthin 1945, Thlipsuridae, card 43; and by Swain 1953,p.272] Devonian, Tennessee.

OFFA [0. barrandiana] Jones 1873b,p.409; Jones and Kirkby 1874, p.53 [Entomoconchidae by U1rich 1894, p.632; and by Pribyl 1951,p.121] Carboniferous, Ireland.

OGMOCONCHA [0. contractula] Triebe1 1941b,p.377 [PlatycopamHealdiidae by Triebell Liassic of Hanover.

OGMOOPSIS [O. nodulifera] Hessland 1949,p.324 [Hollinidae-. Tetradellinae by Hessland; Sigmoopsiidae-Sigmoopsinae by Henningsmpen 1953,p.203,266] Ordovician, Sweden.

OLIGANISCUS Neave 1940, val.3,p.40द, ergor for Dliganisus Geis 1932.

DLIGANISUS [0. suicatus] Geis $1932,0.158$ [Kioedene11idae by Geis; Kloftenellidae-Kloedenfllinas by Henningsmoen 1953,p.242,270) Mississippian of Indiana.

OLIGOCYTHEREIS C Cythereis fullonica Jones and Sherbom 1888, p.256,p1.4,f.13\} Sylvester-Bradiey I948d,p.795 Trachyleberidae-Trachyleberinae by $S \propto B$ J Jurassic, England. 
OMPHALENTOMIS [Cypridina splendens Waldschmidt 1885,p.926, pl.40,f.6a (Given by Kummerow p.62 as Primitia splendens Matern 1939, sic. $=1929, p \cdot 26, p l \cdot 1, f \cdot 5 a-c) 1]$ Kummerow 1953,p.62 [Entomidae by Kummerow] Devonian, Germany. Note: this genus is very much in question as Kummerow in setting it up placed here "Primitia splendens Matern", P. variostriata (Clarke), P. wildungensis Matern, $\underline{P}$. nitida (F. A. Roemer), P. hattingensis Matern, and also possibly p. entomidella Gürich. On p. 62, he compares the genotype of Omphalentomis to $P$. hattingensis, indicating that. they were very close, but the latter was longer. He obviously must have overlooked his own 1939 paper in which he made $\underline{P}$. hattingensis the genotype of Entoprimitial

ONCOCYPRIA [0. mülleri] Daday 1908, p.386-392, 1910a, p.215 $[=$ Oncocypris Müller by Lowndes $1932, p .684,685]$ Recent, German East Africa.

ONCOCYPRIS [0. voeltzkowi] G. W. Müller 1898, p.286 [Cypridae=Cyrpidopsinae by Sharpe 1903,p.990; Cypridae-Cyprinae by G. W. Müller 1912, p.161] Recent, Madagascar.

ONYCHOCYTHERE [O, alosa] Tressler 1939,p.337 [CytheridaeaCytherinae by Tressler] In stomach of a shad, St. Johns River, Florida. 
OPIKATIA [0. emaciata] Kay 1940a, p.264 [Beyrichiidae--

Beyrichinae by Kay; Beyrichiacea of uncertain

family by Henningsmoen 1953,p.274) Ordovician,

\section{Iowa.}

ÖPIKELLA [0. tvaerensis] Thorslund 1940,p.181 [Kirkbyidae by Thorslund; Leperditellidae by Agnew; EurychilinidaeEurychilinfnae by Henningsmoen 1953,p.228,230, 268 ] Ordovician, Sweden.

ÖPIKIUM [Biflabellum tenerum Öpik 1935,p.86,f.40] Agnew 1942, p.759, [new name for Biflabel1um Öp 1 k 1935, not, Doederlein 1913] Ordovician, Estonia.

ÖPIKUM Henningsmoen 1953,p.267 = Öpikium Agnew 1942.

OPISTHOPLAX [0. compressa] Kummerow 1943, P.40 [Primitiidae by Kummerow] Gotlandian, Pomerania.

OROZOE [0. mira] Barrande 1872, p. 537 [ $=$ a Leperditioid Phyllopod by Jones 1883, p.461)

ORTHOCONCHOECIA f for O. haddoni (Brady-Norm.), striosa (CIs.), striola (Müll.) atlantica (Iubb.), and orthotrichota (Miil.), species formerly described under Conw choec1a) Granata and Caporiacco 1949, p.12,15,16,22 [Halocypridae by GEC] Recent, Mediterranean. 
ORTHOCYPRIS [Bythocypris recta Kummerow 1943,p.53,pl.2,f.5, 5a] Kummerow 1953,p.54 [Bairdifdae by Kummerow] Gotlandian, East Prussia.

ORTHONOTACYTHERE [Cytheridea (?) hannai Israelsky 1929, p.12,pl.2A,f.10] Alexander 1933,p.199-200

r "derived from Monoceratina" by Alexander; Cytheridae-Loxoconchinae by Van den Bold 1946 , p.33] Cretaceous, Arkansas.

OSTRACHODA Latreille 1802, vol.3,p.17, Ordre quatrieme [of Entomostracal to include Lynceus, Daphnia, Cypris, and Cythere. As thus used it included both Cladocera and Ostracoda.

OSTRACODA Latreille 1806,p.17, 1810,p.89, a change in spelling of Ostrachoda Latreille 1802.

OSTRACODA Pokorný 1953,p.221,227,230, a Class, composed of the orders Beyrichiida, Podocopida, Myodocopida, and Leperditilida, all new.

OSTRACOPODEN Philippi 1840,p.186, a change in spelling of Ostrapoda Strauss 1821.

OSTRAPODA Strauss 1821, p.58. This is the first time that the "Ostracoda" were separated from the Cladorera and other Entomostraca, and is therefore the first name actually given to the order at present generally termed Ostracoda. Stebbing 1910, p.495, 
and Skogsberg $1920, p .153$ have both forcefully

polnted out that Dstrapoda, rather than Ostracoda, is the proper name for the order. It was used by Desmarest $1925, \mathrm{p} \cdot 380$, and was actually adopted by Latreille himself in 1831 Cours d'Entomologie, p.429. Baird 1850, went back to Ostracoda, and with the exception of Stebbing, later workers have followed suit. It is unjust to Strauss, but it would be difficult to change with so large a body of literature existing in which Ostracoda is used.

OV JCYTHERIDEA [O. nuda] Grekoff 1951, p.56 [Cytheridae-Cytherideinae by Grekoff] Cretaceous of Cameroun. P

PACHEOCIA [P. rodriguesi] Almeida 1950,p.30 [Cypridae by Almelda] Rhaetic or Liassic of Brazil.

PACHYCYPRIS [P. Leuckarti, P. incisa] Claus 1893,P.201

[ E Chlamydotheca Saussure by G. W. Müller 1912, p.183] Recent, Argentina.

PACHYDONELLA [P. tumida] Ulrich 1891, p.197, 198 [Cypridae by Ulrich; Cytherellidae by Bassler and Kellett 1934, p.42; family uncertain, possibly Bary chilinidae by Swain 1953,p.280] Dovonian, Kentucky. 
PAENAEQUINA [P. pentagonalis] Solle 1935,p.44 [Leperditiidae by Solle; Leperditilnae by Henningsmoen 1353, p.274] Devonian, Spitsbergen.

PAIJENBORCHELIA [P. iocosa] Kingma 1948,p.85 [CytheridaeCytherinae by Kingma; Cytheridae-Cytherurinae by Triebel 1949b,p.194] Miocene to Pleistocene in we1l, East Java.

PALEOCOPA Hanningsmoen 1953,p.188,261,266, a suborder to embrace the two superfamilies Beyrichiaced, Ulrich and Bassler, and Leperditacea, Bassler and Kellett.

PALAEOCYPRIS [P. edwardsi] Brongniart 1876,0.49-56,pl.7

[Cypridae by Bassler and Kellett 1934,p.421] Carboniferous, France. [Soft parts preserved].

PALAEOPHILOMEDES [Philomedes bairdiana Jones and Kirkby $1874, p \cdot 43, p l .2, f \cdot 30,31 \mathrm{a}-\mathrm{c} ; \mathrm{pl} \cdot 9, f \cdot 5-8]$ SylvesteraBradley 195la,p.21l. [Rhombinidae by $S-B$ ] Carboniferous of Cork.

PALEOCYTHERE [P. tyna] Tolmachoff 1926,p.36 [Barychilinidae by Bassler and Kellett 1334,p.42I] Devonian, Ellesmereland, Arctic America.

PALMENELIA [Cythereis Iimicola Norman 1865,p.20,pl.6,f.1-4] Hirschmann 1916,p.580 [Cytheridae-Cytherinae by Triebel 1949a,p.188] Recent, North Atlantic and Baltic. 
PARABOLBINA [Ctenobolbina granosa Ulrich 1900,p.183,pl.8, f.12] Swartz 1936,p.570 [Hollinidae by Swartz; Primitiidae-Eurychilininae by Kay 1940a,p.255; Hollinidae-Ctenentominae by Schmidt 1941,p.34; Hollinidae by Henningsmoen 1953,p.270] Devonian, New York.

PARACANDONA [Candona euplectella Robertson 1880, not described; Brady and Norman $1889, p \cdot 105, p 1 \cdot 9, f \cdot 7,8]$ Hartwig 1899a,p.309-31] [Cypridae-Candoninae by Sharpe 1903,p.997] Recent, Scotland and Europe.

PARACAVELLINA [P. elliptica] Cooper 1941,p.37 [Cytherellidae by Cooper] Mississippian of Illinois.

PARACONCHOECIA [P. oblonga,P.spinifera,P.inermis, P.gracilis, ] Claus [1890] 1891,p.13-16 [Halocypridae-Conchoecinae by Claus; = Conchoecia by G. W. Müller 1912,p.59] Recent, Atlantic and Mediterranean.

PARACYPRETTA [P. ampullacea] Sars 1924a,p.152 [Cypridae, nearest to Cypretta by Sars] Recent, South Africa.

PARACYPRIA [Paracypris tenuis Sars 1905,p.404,pl.20,f.173186] Sars 1910,p.732 [Cypridae-Cyprinae-Paracyprides by Sars 1923,p.69] Brackish-water lagoon on the Chatham Islands. 
PARACYPRIDEA [Cypridea (Paracypridea) obovata] Swain 1946b, p.550 [as a subgenus of Cypridea by Swain; as a genus: Cypracea-Cypridae-Cyprideinae by Sylvester-Bradley 1949,p.127,130] Jurassic? Brazil.

PARACYPRIDEIS [Cytheridea fennica Hirschmann 1909,p.286,

1912,p.42] Klie 1929,p.272-274 [Cytheridae-.

Cytherideinae by Van den Bold 1946,p.25] Recent, Baltic.

PARACYPRIDES Sars 1923,p.69, a Group of Cypridae-Cyprinae for Paracypris, Aglaia, Paracypria, and Phlyctenophora.

PARACYPRIDOPSIS [P. zschokkei] Kaufmann 1900c,p.131

[ = Potamocypris Brady, by G. W. Müller 1912,p.214.] Recent, Switzerland.

PARACYPRIS [P. polita] Sars 1866,p.12 [Cypridae- Cyprinae-Paracyprides by Sars 1923,p.69; Cypridae-Candoninae by Bronstein 1939,p.333; Cypridae-Cyprinae by Van den Bold 1946,p.22] Recent, Norway.

PARACYTHERE [P. minima] G. W. Müller 1894,p.285 [Cytheridae, by G. W. Müller 1912,p.262, described in a position between Sclerochilus and Cytherura in the text, but not assigned to a subfamily] Recent, Gulf of Naples, 1 species. 
PARACYTHERE "Ulrich and Bassler" in Bassler 1932,p1.27, for "Paracythere granopunctata," and "P. cornuta," [Kirkbyidae by Bassler and Kellett 1934,p.421; not Paracythere G. W. Müller 1894; both species placed in Graphiodactylus by Morey 1936,p.117. Mississippian of Tennessee.

PARACYTHEREIS [P. impudica] Delachaux 1928,p.63 [Cytheridees by Delachaux] Recent, Peru.

PARACYTHEREIS [P. typicalis] Jennings 1936,p.55 [probably young molts by Van den Bold 1946,p.30; not Paracythereis Delachaux 1928] Cretaceous, New Jersey.

PARACYTHEREIS [Cythere concinna Jones 1857,p.29,pl.4,f.7a-f] Elofson 1941, p.280,288 [As subgenus of Cythereis by Elofson. Invalid as preoccupied, above] Pleistocene, England.

PARACYTHERETTA [P. reticosa] Triebel 1941,p.388 [Cytheridae-Cytherinae by Triebel] Paleocene near Copenhagen.

PARACYTHERIDEA [P. depressa Müller = Cytheropteron bovettensis Seguenza $1880, p \cdot 65, p 1 \cdot 17, f \cdot 54=P$. bovettensis (Seguenza) by Müller 1912,p.305] G. W. Müller 1894, p.340 [Cytheridae-Cytherideinae by Van den Bold $1946, p .26]$ Recent, Mediterranean. 
PARACYTHEROIS [5 species, the first being P. striata Müller = Paradoxostoma flexuosum Brady 1868b, p.461,pl.35, f.30-34 by Sars 1928,p.249-250. This is the best illustrated species and should be the genotype: P. flexuosa (Brady). I have not found any previous designation]. G. W. Miiller 1894,p.324 [Cytheridae-Paradoxostominae by Sars 1928,p.XII] Recent, Atlantic and Mediterranean.

PARACYTHEROMA [P. pedrensis] Juday 1907,p.137 [Cytheridae by G. W. Müller 1912,p.315] In a plankton catch near San Pedro, California.

PARACYTHEROPTERON [Cytheropteron calcaratum Seguenza 1880, p.365,pl.17,f.53,53a = Paracytheridea (Paracytheropteron) calcarata (Seguenza) Ruggieri] Ruggieri 1952b,p.20 [Cytheridae-Progonocytherinae by Ruggieri, as subgenus of Paracytheridea, and intermediate between Paracytheridea and Cytheropteron] Quarternary, Italy.

PARADOXORHYNCHA [P. foveolata] Chapman 1904,p.202, [Cytheridae, near Cytheropteron by Chapman] Jurassic, Western Australia.

PARADOXOSTOMA [P. dispar] Fisher 1855,p.654 [Paradoxostomatidae by Brady and Norman 1889,p.67,229; Cytheridae-Paradoxostominae by G. W. Müller 1894,p.308] 
Monotypic, created because of suctorial nature of mouth. Recent, Madeira.

PARADOXOSTOMATIDAE Brady and Norman 1889, p.67,229, a family of Podocopa for the genera Paradoxostoma and Machaerina.

PARADOXOSTOMINAE G. W. Müller $1894, \mathrm{p} \cdot 308$, a subfamily of Cytheridae for Cytherois, Paradoxostoma, and Machaerina $=$ Xiphichilus.

PARAECHMINA [Cytherina spinosa Ha11, 1852,p.317,p1.67,f.1721 (not Cytherina spinosa Reuss 1846)] Ulrich and Bassler 1923a,p.299 [Primitidae by Bassler and Kellett 1934,p.42l; Aechminidae by Swart, z 1936, p.550; Drepane11idae-Aechmininae by Schmidt 1941, p.63] Silurian, New York.

PARAGRAPHYLUS [P. reticulatus] Coryell and Rosanski 1942, p.144 [Quasillitidae by CER; ?Youngiellidae by Agnew 1942,p.759; Quasillitidae-Quasillitinae by Henningsmoen 1953, p.257,275] Mississippian of Ilinois.

PARAHEALDIA [P. percorella] Coryell and Cuskley 1934, p.3. [Primitidae by CEC; Bairdiidae by Swart. 1936, p.581] Devonian, Oklahoma. 
PARAMEKODON [P. inflatus] Brady and Norman 1896,p.670

$[=$ Pseudophilomedes MülIer 1894, by Muller 1912, p.34] Recent, off Morocco, 1435 metres.

PARAPARCHITELLA [P. ovata] Cooper 1946,p.121 [Leperditellidae by Cooper] Pennsylvanian of Illinois.

PARAPARCHITES [P. humerosus] Ulrich and Bassler 1906,p.149 [Leperditellidae by UGB; Aparchitidae by Schmidt. 1941,p.18; Kloedenellidae-Kloedenellinae by Henningsmoen 1953,p.243,271] Carboniferous, Kansas.

PARAPOLYCOPE [P. germanica] Klie 1936a,p.519-531 [Polycopidae by Klie] Recent, off Helgoland.

PARAPRYBLITES [Pribylites (Parapryblites) hanaicus] Pokorny 1950,p.530,592 [Aparchitidae by Pokorný;

?Alanellidae by Henningsmoen 1953,p.273] Devonian, Czechoslovakia.

PARASCHMIDTELLA [P. dorsopunctata ] Swartz 1936,p.563

[Leperditellidae by Swartz; Aparchitidae by Kay 1940a, p.245; ?Leperditellidae-?Eridoconchinae by Henningsmoen 1953,p.256,275] Devonian of Pennsylvania.

PARASCHULERIDEA [P. anumbonata] Swart $z$ and Swain 1946,p.370 [Cythexidae-Cytherideinae by SES] Jurassic, from Schuler formation in wells, northern Louisiana. 
PARENTHETICA Swartz 1945c, p.1204, possibly intended for Parenthatia Kay, but with two species, $\underline{\underline{p}}$.

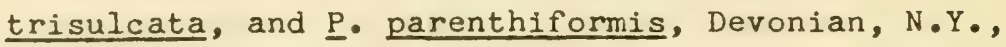
neither described.

PARENTHATIA [Moorea punctata Ulrich 1894, p.682,pl.43,f.84, 88] Kay 1940a,p.259 [Primitiidae-Bollinae by Kay; Drepanellidae-?Bolliinae by Henningsmoen 1953, p.233, 268] Ordovician, Minnesota.

PARULRICHIA [Primitia diversa Jones and Holl 1886b,p.412, p1.14,f.10] Schmidt 1941,p.65 [Drepanellidae-Aechmininae by Schmidt; Drepanellidae-?Bolliinae by Henningsmoen 1953,p.234,268] Silurian, England.

PELLICISTOMA [P. howei] Coryell and Fields 1937,p.17

[Cytheridae-Parádoxostominae by CEF] Miocene of Panama.

PELOCYPRIS [P. lenzi] Klie 1939-1940,I,p.85 [Cypridae-Cyprinae by Klie] Recent, Brazil.

PERISSOCYTHERIDEA [Cytheridea (?) matsoni Stephenson 1935, p.192,193,pl.5,f.1,2,7,8] Stephenson 1938,p.144 [Cytheridae-Cytherideinae by Van den Bold 1946, p.23] Miocene, Louisiana. 
PERPRIMITIA [P. robusta] Croneis and Gale 1939,p.257

[Primitiidae by CEG; Kloedenellidae-Kloedenellinae by Henningsmoen 1953,p.242,271] Mississipptan of Illinois.

PERSANSABELLA [P. bradfieldi] Coryell' and Sohn 1938,p.598 $[=$ Sansabella Roundy by Cooper 1941, p.59; by Agnew 1942,p.759; by Scott 1942,p.154] Mississippian of West Virginia.

PHANASSYMETRIA [P. triserrata] Roth 1929d,p.358-360 [Thlipsuridae by Roth; Cypridae-Cyprinae by Van den Bold 1946,p.22] Devonian, Oklahoma.

PHANASSYMETRICA Neave 19,0, vol.3,p.694, = Phanassymetria Roth 1929d.

PHANASYMMETRICA Van den Bold $1946, \mathrm{p} .22=$ Phanassymetria Roth 1929d.

PHILOMEDES [P. Longicomis l.iljetorg, Recent Skåne a Cypridina brenda Baird $1850, \mathrm{p} .181, \mathrm{pl} .23$ × Philomedes brenda (Baird). Bradvcinetus Sars turned cut to be the female of P. longicornis, or P. ngenda. Liljeborg 1853,p.175 [Cypridinidae-Philomedinae by $G$. W. Müller 1912, p.24] Recent, off N.W. Europe.

PHILOMEINAE G. W. Miiller 1912, p.24, subramily of Cyprjinitae for Fhilomedes, Psmuinphilomedes and Rutiderma. 
PHLYCTENOPHORA [P, zrala:dica] Brady 1880,p.32 [Cypridae by Brady; = Paracypris by G. W. Miller 1912,p.125] Recent, New Zealand.

PHLYCTISCAPHA [P. rockportensis] Kesling 1953b,p.222

[Beyrichiidae by Kesling] Devonian, Michigan.

PHRACTOCYTHERIDEA (Cytheridea (Phractocytheridea) compressa] Sutton and Williams 1939,p.571-573 [as subgenus of Cytheridea by SEW; - Haplocytheridea by Stephenson 1941,p.427.428] Eocene, Texas.

PHREATURA [P. concinna Jones \& Kirkby 1885,p.540, name only] Jones and Kirkby $1886 \mathrm{c}, \mathrm{p} .507$, genus not species; Jones and Kirkby $1887, p .509$, text figs.3,4 genus and species described as new [Leperditildae by JEK; Thlipsuridas by Ulrich 1894,p.632; Healdiidae by Henningsmoen 1953,p.260] Carboniferous, England.

PHYSOCYPRIA [P. bullata] Vávra 1898b,p.7 [as subgenus of Cypria by Vávra; Cypridae-Cyclocypridinae by Sharpe 1903,p.993,994; Cypridae-Candoninae by G. W. Müller 1912,p.132] Recent, East Africa.

PINNATULITES PPrimitiella procera Kummerow 1924,p.419,pl.20, f.12] Hessland 1949,p.198 [Aparchitidae by Hessland; ?Leperditellidae-?Conchoprimitinae by Henningsmoen 1953,p.255,275] Ordovician drift, Prussia. 
PIONOCYPRIS [Cypris vidua O. F. Müller 1776,p.199; 1785, p.55,pl.4,f.7-9] Brady and Norman 1896,p.725 [Invalid, as Brady and Norman 1889,p.89, and Sars $1889 a, p .53$ has already designated Cypris vidua as genotype of Cypridopsis.]

PIRETELLA [P. acmaea] Öpik 1937,p.11] [Piretellidae by Opik; Hollindae-Hollininae by Schmidt 194l,p.32; Tetradellidae-Piretellinae by Henningsmoen 1953, p.217,218,226,267] Ordovician, Estonia.

PIRETELLIDAE Öpik 1937,p.110, a family to include Piretella, Hesperidella, Steusloffia, Pseudostrepula, and Rigidella.

PIRETELIINAE Henningsmoen 1953,p.197,217,267, a subfamily of Tetradellidae to include Piretella, Piretopsis, Rakverella, Dicranella, Ctenotella, and Opikum (sic. $=$ Opikium).

PIRETOPSIS [P. donsi] Henningsmoen 1953,p.218,267 (nude); 1953b,p.43 [Tetradellidae-?Piretellinae by Henningsmoen] Ordovician, Norway •

PLACENTELLA [P. delicata] Wilson 1935,p.636 [Primitiidae by Wilson; Drepanellidae-?Bolliinae by Henningsmoen 1953,p.268. Devonian of Tennessee. 
PLACENTULA [Primitia excavata Jones and Holl 1869, p.222, pl.15,f.10a-c] Jones and Holl 1886b,p.407 [Beyrichiidae by Ulrich 1894,p.632; not Placentula Lamarck 1822: = Jonesites Coryell 1930,p.294, nom.nov.] Silurian, Germany.

PLAGIONEPHRODES [P. uninodosus] Morey 1935a,p.318 [ThIipsuridae by Morey; Quasillitidae-Ropolonellinae by Henningsmoen 1953,p.257,275] Mississippian of Missouri.

PLATELLA [P. gatunensis] Coryell and Fields 1937,p.3 [Cytherellidae by CEF] Miocene, Panama.

PLATYBOLBINA Henningsmoen 1953,p.227,228,268, nom•nov. for Platychilina Thorslund 1940,p.169, non Koken 1892, Genotype: Primitia distans Krause 1889,p.6,pl.1, $\mathrm{f} \cdot 3 \mathrm{a}, \mathrm{b}=$ ? Primitia plana Krause, by Henningsmoen 1954, p.87. Ordovician drift, Germany.

PLATYCHILELLA [Platychilus ovoides Cooper 1941,p.38,pl.6, f.26-27] Cooper 1942,p.777, new name for Platychilus Cooper 1941, not Jakolev, 1874 [Cytherellidae by Cooper; Beyrichiidae by Agnew 1942,p.760; ?Healdiidae by Henningsmoen 1953, p.260] Mississippian of Illinois. 
PLATYCHILINA Kummerow 1933,p.45 [Primitia distans Krause figured as a Platychilina, p.44, but not designated].

PLATYCHILINA [Primitia elongata Krause 1891,p.494,pl.30, f.4a,b] Kummerow 1939,p.19 [Primitiidae-Eurychilininae by Kummerow; Not Platychilina Koken 1892 , by Agnew 1944,p.219] Ordovician to Permian by Kummerow.

PLATYCHILUS [P. ovoides] Cooper 1941,p.38 [Cytherellidae by Cooper; not Platychilus Jakolev 1874 Platychilella Cooper 1942,p.777] Mississippian of Illinois.

PLATYCOPA Sars 1866, p.124 for the family Cytherellidae and genus Cytherella. Triebel 1950b,p.117, adds the Healdiidae to the Platycopa.

PLATYCYTHEREIS [Cythereis excavata Chapman and Sherborn 1893, p.348,pl.14,f.8] Triebel 1940,p.214 [CytheridaeCytherinae by Triebel] Cretaceous, England.

PLEOSCHISMA [P. robusta, P. moroides, P. reticulata] Brady 1890,p.513 [Cypridinidae by Brady; = Philomedes by G. W. Müller 1912,p.25; certainly valid genus : Cypridinidae-Philomedinae by Skogsberg 1920, p.348] Recent, South Pacific. 
PLETHOBOLBINA [P. typicalis] Ulrich and Bassler 1923a, p.307, $1923 b, p .635$ [Zygobolbidae-Kloedeninae by UGB; Beyrichiidae-Zybogolbinae by Henningsmoen 1953, p.238,269] Silurian, West Virginia.

PLEUROCYTHERE [P. richteri] Tricbel 1951,n.88 [Cytheridae-Cytherinae by Triebel] Jurassic, Gexmany.

PODOCOPA Sars 1866, p.10, a suborder of Ostracoda for the family Cypridae, with the genera Cynris, Cypria, Paracypris, Notodromas, Candona, Pontocypris, Argilloecia, and Bairdia; and the family Cytheridae, with the genera Cythere, Cythereis, Cyprideis, Cytheridea, Cytheropsis, Ilyobates, Loxoconcha, Xestoleberis, Cytherura, Cytheronteron, Bythocythere, Pseudocythere, Sclerochilus, and Paradoxostoma.

PODOCOPA Sars 1928, D. IX-XII, a suborder of Ostracoda for the family Cypridae, with subfamilies Pontocyprinae, Nacrocyprinae, Bairdiinae, Cvprinae (with 7 groups), Darwinulinae; and Cytheridae, with subfamilies Limnocytherinae, Cytherideinae, Cytherinae, Cytherurinae, Loxoconchinae, Bythocytherinae, Xestoleberinae, Paradoxostominae.

PODOCOPIDA Pokorný 1953,p.222,227,231, an order of Ostracoda. "It comprises the suborders Platycopa G. O. Sars 1866, and Podocopa G. O. Sars 1866." 
POLONIELLA [P. devonica] Gürich 1896,p.388 [Thlipsuridae by Bassler and Kellett 1934,p.433; Kloedenellidae-Kloedenellinae by Henningsmoen 1953,p.242,261,275] Devonian, Poland.

POLYCERATELLA [Ulrichia kuckersiana Bonnema 1909,p.51,pl.6, f.10-15] Öpik 1937,p.10] [?Hollinidae-Tetradellinae by Schmidt 1941,p.37; Beyrichidae by Agnew 1942, p.760; Tetradellidae-?Tetradellinae by Henningsmoen 1953,p.267] Ordovician, Estonia.

POLYCHELES [P. stevensoni] Brady and Robertson 1870,p.25 [non Polycheles Heller $1862=$ Narwinella Brady and Robertson $1872, p \cdot 50$, nom.nov., also preoccupied $=$ Daminula Brady and Robertson, nom.nov., in Jones $1885 e, p \cdot 346$ ] Recent, Europe.

POLYCOPE [P. orbicularis] Sars 1866,p.121 [Polycopidae by Sars] Recent, off Norway.

POLYCOPIDAE Sars 1865, p.121, a family of Cladocopa, for the genus Eolycope. Sars 1928,p.IX added the genus Polycopsis, Müller 1894 .

POLYCOPIFORME: ikogsberg $1920, \mathrm{p} .158$, a suborder nfor all Polycopids." " Cladocopa Sars 1866.

POLYCOPSIS [Polycope compressa Rrady and Robertson 1869, p.372,p1.21, f.5-11, designated by Sars 1923,p.37] 
G. W. Müller 1894,p.238 [Polycopidae by Sars $1923, \mathrm{p} .36$; 1928,p.IX] Recent, off Ireland.

POLYPHYMA [P. Iapworthi] Groom 1902,p.84-86 [described as an ostracod; Conchostraca by Ulrich and Bassler 1931, p.64] Cambrian, England. [not Polyphyma Jakolev, 1877, Hem.; nor Hamm 1881, broyz., fide Neave 1939, vol.3,p.860.

POLYTYLITES [P. geniculatus] Cooper 1941,p.5] [Kirkbyidae-Amphissitinae by Cooper] Mississippian of Illinois.

POLYZYGIA [P. symmetrica] Gürich 1896,p.387 [Leperditidae by Gürich; Zygobolbidae-Drepanellinae by Bassler and Kellett $1934,0.435$; ?Drepanellidae-Bassleratilnae by Schmidt 1941,p.50; Beyrichiacea of uncertain family by Henningsmoen 1953,p.274] Devonian, Poland.

PONDERODICTYA [Cytherella (?) bispinulatus stewart 1927, $\mathrm{p} \cdot 60, \mathrm{pl} \cdot 5, \mathrm{f} .18,19=$ Ponderodictya bispinulata (Stewart)] Coryel1 and Malkin 1936,p.15 [Cytherellidae by CEM; Healdiidae by Henningsmoen 1953, p.257, 260,261] Devonian of Ontario.

PONTOCYPRIA [P. spinosa] G. W. Müller 1894,P.255 [Cypridae-Pontocyprinae by Miller] Recent, Gulf of Naples. 
PONTOCYPRIDAE Alm 1915,p.27, a "Gruppe" of Cypridae for the genera Argilloec1a, Pontocypria, Erythrocypris, and Pontocypris.

PONTOCYPRIDINAE Kaufmann 1900a,p.108, subfamily of Cyprididae to include Pontocypris, Erythrocypris, Pontocypria, and Argilloecia.

PONTOCYPRINAE G. W. Müller 1894,p.246, subfamily of Cypridae, for the genera Pontocypris, Pontocypria, Erythrocypris, and Argilloecia.

PONTOCYPRIS [ 3 genosyntypes: P. serrulata, P. hispida, P. trigonella. Cythere (Bairdia) mytiloides Norman $1862 a, p \cdot 50, p 1 \cdot 3, f \cdot 1-3$ was designated by Brady and Norman 1889,p.107, with P. serrulata as a synonym] Sars 1866 [Cypridae-Pontogyprinae by Mü1] er 1894, p.246] Recent, off northwestern Europe. The designation of Cythere mytiloides by Sars 1923, p.50-51, as genotype of Eirythrocypris, and by Sars 1923,p.48 of P. trigonella as genotype of Pontocypris as therefore both invalid. It means that the species given by G. W. Miiller 1912, and Sars 1923 as Erythrocypris belong to Pontocypris, and those they considered Pontocypris as exempli-

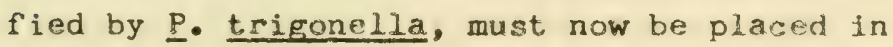
Propontocypris Sylvester-Bradley $1947, p .193$. 
PONTOCYTHERE [P. tchernjawskii] Dubowsky 1939,p.25 [Compared to Cyprideis and Cytheridae by Dubowsky] Recent, Black Sea.

PONTOPARTA [P. rara] Vávra 1901,p.184 [Cyprididae-Cyclocypridinae by Sharpe 1903, p.995; Cypridae-Candoninae by G. W. Muller 1912,p.126] Recent, Bismark Archipelago.

PORACYPRIDOPSIS Kaufmann 1900b,p.316, typographical error for Paracypridopsis.

POTAMOCYPRIS [Bairdia fulva Brady $1868 \mathrm{~b}, \mathrm{p} .474, \mathrm{pl} .28, \mathrm{f} .21$ ] Brady $1870, p \cdot 365,366$ [as subgenus of cyprig by G. W. Muller 1900,p.86; as subgenus of Cypridopsis by Alm 1915,p.83; and by Furtos 1933,p.432; Cyprididae-Cypridopsinae by Kaufmann 1900a,p.106; Cypridae-Cyprinae-Cypridopsini by Bronstein 1947, p.154] Recent, British Isles.

PŘIBYLITES [P. moravicus] Pokorný 1950,p.527,589 [Aparchitidae by Pokorný; ?Alanellidae by Henningsmoen 1953,p.248, 273] Devonian, Czechoslovakia.

PRIMITIA [27 species and several varieties, the first, Beyrichia strangulata Salter 1855 was designated genotype by Miller 1889,p.561] Jones and Holl 1865, p.415 [Beyrichiidae by Ulrich 1894,p.632; 
Leperditiidae-Aparchitinae by Jones in Chapman 1901,p.147; Primitidae by Bassler and Kellett. $1934, p .438$, and by Henningsmoen 1953,p.266, 267] Ordovician, British Isles. Several hundred specific names have been applied to this genus.

PRIMITIELLA [P. constricta] Ulrich 1894,p.647 [Beyrichildae by Ulrich; Primitildae-Primitiinae by Bassler and Kellett 1934,p.17; Eurychilinidae-?Euprimitiinae by Henningsmoen 1953,p.229,268] Ordovician, Kentucky •

PRIMITIIDAE Ulrich and Bassler 1923a,p.297, a family of Beyrichiacea, to include Primitiella, Haploprimitia, Primitia, Laccoprimitia, Euprimitia, Halliella, Primitiopsis, Ulrichia, Bollia, Placentula, Jonesella, Dicranella, Bolbibollia, Aechmina, Paraechmina, Acronote1la, Dilobella, Bursule11a, with the subfamily Eurychilininae, to include Eurychilina, Coelochilina, Chilobolbina, Apatochilina, Apatobolbina.

PRIMITIIDAE of Swartz $1936, \mathrm{p} .549$, reduced to include Primitia, Primitie1]a, Haploprimitia, Laccorprimitia, Euprimitia, Halliella, Hallatia, Pyxiprimitia, ?Milleratia, Jonesites, and Hippa. 
PRIMITIIDAE of Schmidt $1941,0.25$ divided into subfamilies Primitisinae, Eurychilinae, Primitiopsinae.

PRIMITINAE Bassler and Kellett 1934,P.16, a subfamily of Primitiidae to Include: Primitielia, Haploprimitia, Primitia, Laccoprimitia, Euprimitia, Halliella, Primltiopsis, Ulrichia, Bollia. Jonesites, Jonese11a, Dicrane11a, Bolbibollia, Aechmina, Paraechmina, Acronotella, Bilobella, Bursulella, Monoceratina, Mooreina. This subfamily is greatly reduced by Bouček 1936,p.41, and by Schmidt 1941, p.25, and is felt unnecessary by Henningsmoen 1953, who reduces the entire family Primitiidae to 4 genera.

PRIMITIOPSIDAE Swart: $1936, \mathrm{p} \cdot 555$, a family for the genus Primitiopsis.

PRIMITIOPSINAE Hessland $1949, \mathrm{p} .239$, a subfamily of Primitildae, for the genus Primitiopsis.

?PRIMITIOPSIINAE Henningsmoen 1953,p.197, 230, 268, as a subfamily of Eurychilinidae, for the genus Primitiopsis.

PRIMITIOPSIS [P. planifrons] Jones 1887, p.5 [Beyrichiidae by U1rich 1894,p.632; Cytheridae by Jones, in Chapman 1901,p.147; Primitiidae-Primitilnae by Bassler and Kellett 1934,p.17; Primitiopsidac by 
Swartz 1936,p.555; Primitiidae-Primitiopsinae by Hessland 1949,p.239; Eurychilinidae-Primitiopsiinae by Henningsmoen 1953,p.268] Silurian, Gotland.

PRIONOCYPRIS [Candona serrata Norman 1862a,p.46,pl.2,f.1-6; $1862 \mathrm{~b}, \mathrm{p} \cdot 146, \mathrm{pl} \cdot 3, \mathrm{f} \cdot 1-6=$ Cypris zenkeri Chyzer and Toth, in Chyzer, 1858,p.514, by Lowndes 1931, p.93-97, who redefines genotype] Brady and Norman $1896, \mathrm{p} \cdot 724$.

PRIONOCYTHERETTA [P. prima] Méhes 1941,p.60 [ $=$ Cytheretta by Triebel 1952, p.16] Oligocene, Hungary.

PROCYTHERIDEA [P. exempla] Peterson 1954,p.171, Cytheridae, near Haplocytheridea, and Protocythere by Peterson]. Jurassic, Wyoming.

PROCYTHEREIS [Cythereis (Procythereis) torquata] Skogsberg $1928, p .17$ [a subgenus of Cythereis by Skogsberg] Recent, Tierra del Guego.

PROGONOCYTHERE [P. stilla] Sylvester-Bradley $1948 \mathrm{~b}, \mathrm{p} .189$ [Cytheridae-Progonocytherinae by S-B] Jurassic, England.

PROGONOCYTHERINAE Sylvester-Bradley 1948b,p.189, a subfamily of Cytheridae to include Progonocythere and Lophow cythere. 
PROPARAPARCHITES [P. ovatus] Cooper 1941,P.62 [Leperditellidae by Cooper; Kloedenellidae-Kloedenellinae by Henningsmoen 1953,p.243,271] Mississippian of Illinois.

PROPLECTRUM [P. platum] Kesling and McMillan 1951,p.64 [Hollinidae by Kesling and McMillan, Devonian, Michigan.

PROPONTOCYPRIS [Pontocypris trigonella Sars 1866,p.16] Sylvester-Bradley 1947,p.193, Jnew name for Erythrocypris Müller 1894,p.256; 1912,p.109, Sars 1923,p.47; Cypridae-Pontocyprinae] Recent off Northwestern Europe.

PROTEOCYPRIS [P. salina] Brady 1906,p.334[= Cypridopsis Brady, by G. W. Müller 1912,p.208] Recent, England.

PROTOCYTHERE [Cytherina triplicata Roemer 1840,p.104,pl.16, f.16] Triebel 1938b,p.180 [Cytheridae-Cytherinae by Triebel] Lower Cretaceous, Germany.

PRUVOSTINA [P. wanlessi] Scott and Summerson 1943,p.670 [Cypridae by SES] Pennsylvanian; Tennessee.

PSAMMOCYTHERE [P. remanei] Klie 1936b,p.59 [Bythocytherinae by Klie, 1936b; Cytheridae-Psammocytherinae by KIie 1938a,p.214] Recent, Helgoland. 
PSAMMOCYTHERINAE Klie 1938a,p.214, a subfamily of Cytheridae for the genus Psammocythere.

PSEUDOCANDONA [Cypris pubescens Koch ]837,p.146,6; = Candona pubescens of Hartwig 1899b,p.186,f.2 $=$ Candona insculpta G. W. Müller 1900,p.28,pl.3,f.15,16,21, 22; pl.4,f.1,7-10 (not Candona pubescens (Koch) of Müller 1900,p.26,pl.4,f.3,4,6,16,18,19,21,22) by Lowndes $1931, p \cdot 98-102$, figs.67-81;p1.3,f.1015] Kaufmann $1900 \mathrm{~b}, \mathrm{p} .375$, in a footnote, though pictured,p1.27,28,31, as Candona pubescens Sars. [Kaufmann gave no diagnosis of his genus and it remained for Lowndes 1931 to revive it. Bronstein 1939,p.333 recognized it and placed his own Metacandona in the synonymy of Pseudocandona, and in 1947,p.188-205 gave a thorough discussion of the genus with numerous species. Cypridae-Candoninae-Candonini by Bronstein] Recent, Europe and Asia.

PSEUDOCONCHDECIA [Conchoecia serrulata Claus 1874,p.6,pl.1, fீ.2-7,9-11,pl.2,f0.12-13,17,19] Claus [1890] 1891, p.20 [Halocypridae-Conchoecinae by Claus; = Conchoecia by G. W. Müllex 1912,p.81] Pacific, At lantic and Indian oceans.

PSEUDOCYPRETTA [P. maculata] KIie 1332,p.484 [Cypridae bv KIie] Recent, Sumatra and Java. 
PSEUDOCYPRIDINA [P. piedmonti] Roth 1933,p.404,405

[ = Cypridea by Harper and Sutton 1935,p.625; as

subgenus of Cypridea by Sylvester-Bradley 1949 ,

p.126; as valid genus of Cypridae-Cyprideinae by

Peck 1951,p.318,319] Jurassic, South Dakota.

PSEUDOCYPRIS [P. bouvieri] Daday 1908,p.317; Daday 1910, p.193 [Cypridae, very close to Cypris by Sars 1924,p.177-178] Recent, German East Africa.

PSEUDOCYPROIDES [P. alatus] Morris and HiIl 1952,0.15

[Bairdiidae by MEH, who compared it to Pseudocypris] Silurian, Tennessee.

PSEUDOCYTHERE [P. caudata] Sars 1866,p.87 [Cytheridae-Bythocytherinae by Sars 1926,p.232-233] Recent, North Atlantic.

PSEUDOCYTHEREIS [Cythereis (Pseudocythereis) spinifera] Skogsberg 1928,p.126 [as subgenus of Cythereis by Skogsberg, but compared by Skogsberg-to Cytheretta and Hemicythere] Recent, South Georgia.

PSEUDOCYTHEREIS [P. reticulata] Jennings 1936,p.56 [Preoccupied by Pseudocythereis Skogsberg =Anticythereis Van den Bold 1946,p.30, nom.nov.] Cretaceous, New Jersey.

PSEUDOCYTHERETTA [P. edwardsi] Cushman 1906,p.382 [" Cytheret.ta by G. W. Müller 1912,p.366] Recent, Massachusetts. 
PSEUDOCYTHERURA [P. pontica] Dubowsky 1939,p.12,50,51

[Cytheridae-Cytherurinae by Dubowsky] Recent, Black Sea.

PSEUDOENTOMOZOE [Entomis pteroides Canayari 1899,p.151:

lectotype selected by Pribyl as specimen figured

by Canavari 1900,pl.26,f.4] Proby 1 1951,p.116

[Entomozoacea-Entomzoidae-Entomozoinae by Pribyl]

Silurian, Sardinia.

PSEUDOKRITHE [P. dictyote] Méhes 1941a,p.20 [Cytheridae by Méłes] Oligocene, Hungary.

PSEUDOLIMNOCYTHERE [P. hypogaea] KIie 1938b,p.150 [Compared to Limnorythere by KIie] Subsurface, Italy.

PSEUDOLOXOCONCHA [P. minima] G. W. Mül1er 1894,p.348

[Cythesidae by G. W. Müller 1912,p.314] Recent, Gulf of Naples.

PSEUDONODELLA [P. plana] Zaspelova 1952,p.193 [Drepanallidae-Nodellinae by Zaspelova] Devonian, USSR.

PSEUDOPARAPARCHITES [P. kansasensis] Ke11ett 1933,p.67

[Aparchitidae by Kellett; Leperditellidae by Bassler and Kellett 1934,p.466; Kloedenel11daeKloedene1linae by Henningsmoen 1953,p.242,271] Permian of Kansas. 
PSEUDOPHANASYMMETRIA [Phanassymetria foveata Veen 1936b, p.47(177),pl.10,f.16-22] Sohn and Berdan 1952, p.10 [Bairdiidae by Sohn and Berdan] Cretaceous, Limburg.

PSEUDOPHILOMEDES [P. foveolata, P. angulata; the first should be the type] G. W. Miuller 1894,p.21I [Cypridinidae-Philomedinae by G. W. Müller 1912, p.24] Recent, Gulf of Naples.

PSEUDOSTREPULA [Strepula kukersiana Bonnema 1909,p.49,pl.6, f.16-23] Öpik 1937, p.116 [Piretellidae by Öpik; ?Hollinidae-Tetradellinae by Schmidt 1941,p.37; Tetradellidae-Bassleratiinae by Henningsmoen 1953 , p.220,267] Ordovician, Estonia.

PSEUDULRICHIA [Leperditia bivertex Ulrich 1879,p.11,pl.7, f.5,5a] E.A.Schmidt 1941,p.59 [Drepanellidae-Ukrichiinae by Schmidt] Ordovician, Kentucky.

PTERIGOCYTHEREIS Van den Bold 1946,p.29 = Pterygosythereis Blake 1933.

PTEROCODELLA [P. mirabilis] Croneis and Gale 1939,p.260 $[=$ Tetrasacculus Stewart 1936, by Cooper 1941, p.63] Mississippian of Illinois.

PTERYGOCYTHEREIS [Cythereis jonesi Baird 1850a,p.175,pl.20, fig.I] Blake 1933,p.239 [As a subgenus of Cythereis 
by Blake; as a genus by Triebel 1941,p.385-386;

Trachyleberidae by Sylvester-Bradley 1948,p.793]

Recent, North Atlantic.

PULLVILLITES [P. triangulum] Öpik 1937,p.119 [Cypridacea by Öpik; ?Acronotellidae by Agnew 1942,p.760] Ordovician, Estonia.

PUNCIA [P. novozealandica] Hornibrook 1949,p.470 [Punciidae, resembling Eurychilininae of the Paleozoic by Hornibrook; Beyrichiacea? - Puncildae by Hennings moen 1953,p.235] Recent, New Zealand.

PUNCIIDAE Fornibrook 1949,p.469-471, a living family of ostracods with strong resemblance to Paleozoic Beyrichiacea, for the genera Puncia and Manawa.

PUNCTAPARCHITES [Cytheropsis rugosus Jones 1858,p.249a,pl.10, f.5] Kay 1934,p.331 [ Macronote1]a by Kay 1940a, p.245] Ordovician, Canada.

PUNCTOPRIMITIA [Haploprimitia simplex stewart 1936, p.743, pl.100,f.5] Stewart and Hendrix 1945a,p.90 [Primitiidae by SGH; Beyrichiacea of uncertain family by Henningsmoen 1953,p.229,248,274 ] Devonian, Ohio.

PURIANA Coryell and Fields, by Coryel1, in Puri 1953b,p.751, new name for Favella Coryell and rields 1937, not Jorgensen 1925. 
PYRICYTHEREIS [Cythereis?issaelsky 1 Howe and Pyeatt, in Howe and Chambers 1935,p.33, pl.1,f.19-2l;pl.4, f.7-9] Howe, in Howe and Law 1936, p.65 [ Butonia Howe, by Howe 1947,p.50, and by Stephenson 1947 , p.579] Eocene, Louisiana.

PYROCYPRIS [6 species, the first P. chierchiae] G. W. Müller 1890a, p.230-232 [Cypridinidae by G. W. Müller; Cypridina (Cypridina) by Skogsberg 1920,p.313317] Recent, Arabian sea.

PYXION [Primitia carinata Hadding 1913,p.68,pl.6,f.12] Thorslund 1948,p.367 [Family not given by Thorslund; Beyrichiacea of unknown family by Henningsmoen 1953,p.274] Ordovician, Sweden.

PYXIPRIMITIA [P. ventriclefta] Swartz 1936,p.568 [Primitiidae by Swartz; Drepanellidae-Ulrichiinae by Schmidt 1941,p.51; Beyrichiacea of uncertain family by Henningsmoen 1953, p.229,261,275] Devonian, Pennsylvania.

QUADRACYTHERE [Cythere truncula Brady 1898,p.444,p1.47, f.16,17] Hornibrook 1952,p.43 [CytheridaemTrachyleberinae by Hornibrook] Recent, New Zealand. 
QUADRIJUGATIDAE Kesling and Hussey 1953,p.79, a family of Beyrichiacea for Quadrijugator, Ceratopsis, Glossopsis, Ogmoopsis, and a portion of "Tetradella". QUADRIJUGATOR [Bollia permarginata Foerste 1918, p.124-125, pl.6, f.33a-c] Kesling and Hussey 1953,p.82 [Quadrijugatidae by KEH] Ordovician-Silurian of Michigan.

QUASILI.ITES [Q. obliquus] Coryell and Malkin 1936,p.18

[Quasillitidae by CEM; Graphiodactyllidae by Agnew 1942, p.760; Quasillitidae-Quasillitinae by Henningsmoen 1953,p.255,257,275] Devonian, Ontario. QUASILLITIDAE Coryell and Malkin 1936,p.17, a family to include Quasillites, Janetina, Jenningsina.

QUASILIITIDAE Henningsmoen 1953,p.256,261,275, a family of Podocopa to include the subfamilies Quasillitinae and Ropolonellinae.

QUASILIITINAE Henningsmoen 1953,p.257,275, a subfamily of Quasillitidae to include Graphiodactyllis, Quasillites, Spivonina, Janetina, Jenningsina, Bufina, Paragraphylus, and ?Augurites.

R

RAKVERELIA [R. spinosa] Öpik 1937,p.109 [Beyrichiidae by Öpik; Piretellidae by Agnew 1942,p.760; Hollinidae-Ctenonotellinae by Schmidt 194l, p. 48; 
Tetradellidae-?Piretellinae by Henningsmoen 1953, p.218,267] Ordovician, Estonia.

RANAPELTIS [R. typicalis] Bassler 1941,p.26 [Thlipsuridae by Bassler] Devonian, Tennessee.

RAYELLA [Basslerites hanseni Teichert 1937,p.117,pl.23, f.11-15] Teichert 1939,p.622, new name for Basslerites hanseni Teichert 1937,p.117, not Basslerites Howe, November 1, 1937. [Beecherellidae by Teichert, also by Kay 1940a,p.267, and Agnew 1942,p.760] Ordovician, Greenland.

RAYMONDATIA [R. goniglypta] Kay 1934,p.342-342 [Beyrichiidae by Kay; Tetradellidae by Swartz 1936,p.551; ?Beyrichiidae-Piretellinae by Kay 1940a, p.266; Drepanellidae-Bassleratiinae by Schmidt 1941,p.50; Tetradellidae-Bassleratiinae by Henningsmoen 1953, p.219,267] Ordovician, Iowa.

RECTOTRACHYLEBERIS [Cythereis hamata Müller 1894,p.373,pl.19, 31] Ruggieri 1952b,p.38 [n.subgen of Cythereis] Recent.

RETICESTUS [R, acclivitatus] Kesling and Weiss 1953,p.52 [Kirkbyidae by KEW] Devonian, Michigan.

REVERSABELLA [R.njorthi] Coryell and Johnson 1939,p.221

[ Sansabella Roundy by Cooper 1941,p.57,60; by Agnew 1942,p.760; by Scott 1942,p.154] Mississippian of Illinois. 
RHINOCYPRIS [R. scabra] Anderson 1940,p.378 [Closely related to Scabriculocypris and through it to Metacypris by Anderson] Jurassic, England.

RHOMBINA [R. hibernica Jones and Kirkby $1874, \mathrm{p} .44, \mathrm{pl} .2$, f.33; pl.5,f.13, designated by Bassler and Kellett 1934,p.45] Jones 1873b,p.41] [Cypridinidae by Jones; Rhombinidae by Sylvester-Bradley 1951a, p.209] Carboniferous, Ireland. [Sylvester-Bradley 1951 a restudied, refigured and reoriented Jones and Kirkby's material, and redefined the genus Rhombina, and corrected his designation (1951b)].

RHOMBINIDAE Sylvester-Bradley 1951a,p.209, a family of Myodocopa for the genera Rhombina and Palaeophilomedes.

RHOMBOENTOMOZOE [Cryptocaris? rhomboidea Barrande 1872, p.464,pl.31,f.14,15] Pribyl 1951,p.11] [Entomozoacea-Entomozoidae-Entomozoinae by Pribyl] Silurian, Bohemia.

RICHINA [R. truncata] Coryell and Malkin 1936,p.3 [Beyrichiidae by CGM; Tetradellidae by Agnew 1942, p.760; Drepanellidae-?Bolliinae by Henningsmoen 1953,p.234,268] Devonian, Ontario. 
RICHTERIA [Genotype not given, but used for the "Cypridinen" described by Dr. Richter 1856] Jones 1874,P.180; but abandoned by him as unjustified and = Estomis 1879,p.183. Not Richteria Girault 1920, p.2, Hym., fide Neave 1939, vol.4, p.74.

RICHTERIA Jones, Kegel 1934,p.413, revived as a subgenus of Entomis, with Entomis serratostriata (Sandberger) $1845[$ = Cypridina serrato striata Sandberger $1845,2.121,123, \mathrm{pl} .1, f .6$ designated by Kegel as subgenotype. Devonian, Germany] [ s subgenus of Entomozoe by Pribyl 1951,p.105].

RICHTERINA [Cytherina costata Richter 1869,p.773,p1.21, f. 8, 9] Gürich 1896,p.377 [Entomidae by Bassler and Kellett 1934,p.467; Entomozoacea-Entomozoidae-Entomozoinae by Přibyl 1951,p.105] Devonian, Germany.

RIGIDELLA [Steusloffia mitis] Dpik 1937,p.117 [Piretellidae by Öpik; = Tetradella by Hessland 1949, p. 340; Hollinidae-Tet radellinae by Schmidt 1941,p.37; Tetradellidae-Bassleratiinae by Henningsmoen $1953, \mathrm{p} \cdot 220,221,267$.

RIOCYPRIS [R. uruguayensis] K1ie 1935b, P. 289 [Cypridae by KIie] Recent, Uruguay. 
ROPOLONELLIDAE COrye $\lambda \AA$ and Malkin $1936, \mathrm{p} .6$, a family to include Ropolonellus, Rudderina, Euglyphella, and Bufina.

ROPOLONELLINAE Henningsmoen 1953, a reduction of Coryell and Malkin's family to a subfamily of Quasillitidae, for the genera Ropolonellus, Euglyphella, Rudderina, and Plagionephrodes.

ROPOLONELLUS [P. papillatus] Van Pelt 1933,p.339 [ThIipsuridae by Van Pelt; Ropolonellidae by Coryell and Malkin 1936,p.6; QuasillitidaewRopolonellinae by Henningsmoen 1953,p.257,260,275] Devonian, Michigan.

ROSTOCYPRINAE Anderson 1939,p.293, a subfamily of Cypridae, to include Cypridea, Cyamocypris, Langtonia, Morinina, Ulwellia. Not named in accordance with RULES, as not based on a genus with similar name. $=$ Cyprideinae Martin 1940,p.280,281.

ROTHELLA [Dizygopleura recta Roth 1929d,p.344,pl.36,f.8a-c] Wilson 1935,p.643 [ThIipsuridae by Wilson] Devonian, Oklahoma.

ROUNDYELLA [R. simplicissimus Knight 1928,p.266,pl.32,f. 1la-d] Bradfield 1935,p.66 [Kirkbyidae by Bradfield] Pennsylvanian, Missouri. 
RUDDERINA [R. extensa] Coryell and Malkin 1936,p.6

[Ropolonellidae by CGM; Quasillitidae-Ropolonellinae by Henningsmoen 1953,p.257,275] Devonian of Ontario.

RUPTIVELUM [R. bacculatum] Kesling and Weiss 1953,p.47 [Hollinidae by KEW] Devonian, Michigan.

RUSSIA [Gravia (Russia) unicostata] Polenova 1952,p.86 [as a subgenus of Gravia: Acronotellidae-Graviinae by Polenoval Devonian, USSR.

RUTIDERMATIDAE Brady and Norman $1896, p .673$, a family of Myodocopa for the genus Rutiderma.

RUTIDERMA [R. compressa] Brady and Norman 1896,p.673

[Rutidermatidae by BGN; Cypridinidae-Philomedinae by G. W. Müller 1912,p.34] Recent, Bay of Biscay.

RUTTENELLA [R. ovata] Bold 1346,p.84 [Cytheridae-Cytherideinae by Van den Bold] Eocene of Bonaire.

SACCALATIA Kay 1940a,p.242, as spelled Saccelatia 5 t.imes on p.238, and same 5 times on $p_{0} 243=$ Saccelatia Kay 1940a; corrected by Kay 1940b, p.615.

SACCEIATIA [Aparchites arrectus U1rich $1894, \mathrm{p} .646, \mathrm{pl} .43, \mathrm{f} .35-$ 36) Kay 1940a,p.242 (spelled Saccalatia in typographical error on this pape only; Saccelatia in 
10 other places in paper,p.238,243) (Leperditellidae by Kay;?Aparchitidae by Agnew 1942,p.760; Drepanellidae-?Bolliinae by Henningsmoen 1953,p.233, 269] Ordovician, Minnesota.

SACCULUS [Cythereis (Sacculus) trigibbosus, C. (S) tetragibbosa] Neviani 1928,p.72 [a "Gruppo" = subgenus or genus of Cythereis] Fossil, Italy. [not Sacculus Gosse 1851,verm., nor Hirase 1927, moll., fide Neave 1939, vol.4,p.96].

SAFFORDELLA [S. muralis] Ulrich and Bassler 1923,p.295 [Preoccupied by Saffordella Dunbar 1920, moll. = Saffordellina Bassler and Kellett 1934,p.14,470] Ordovician, Tennessee.

SAFFORDELIINA Bassler and Kellett 1934,p.14,470, new name for Saffordella Ulrich and Bassler,1923, not Dunbar 1920 [Leperditiidae by Bassler and Kellett; Leperditiidae-Isochilininae by Henningsmoen 1953, p.256,274].

SAHNIA [Cytherideis subulata Brady 1868,p.454,pl.35,f.43-46] Puri 1952,p.912 [Cytheridae-Cytherideisinae by Puri; = Neocytherideis Puri by Sylvester-Bradley and Harding 1953,p.755] Recent, British Isles.

SAIDA [S. truncala] Hornibrook 1952,p.67 [Cytheridae-Incertae sedis by Hornibrook] Recent, New Zealand. 
SAMARELLA [S. crassa] Polenova 1952,p.136 [Incertae Sedis by Polenova] Devonian, USSR.

SANSABELLA [S. amplectans] Roundy 1926,p.5 [Aparchitidae by Roundy; Glyptopleuridae by Geis 1932,p.174; Leperditellidae by Bassler and Kellett 1934,P.470; ?Aparchitidae by Schmidt 1941,p.18,71; Kloedene1lidae-Kloedenellinae by Henningsmoen 1953,p.196,242,270] Pennsylvanian, Texas.

SANSABELLOIDES [Jonesina texana Warthin 1930,F.60,p1.4, f.10 (not Jonesina texana Harlton 1929] Harris and Lalicker 1932,p.401. [Kloedenellidae by Harris and Lalicker = Sulcella by Bassler and Kellett 1934, p.470,478] Carboniferous, Kansas.

SARGENTINA [S. allani] Coryel1 and Johnson 1939,p.223

[Cytherellidae by CGJ; KIoedenellidae by Cooper 1946,p.117; Kloedenellidae-Kloedenellinae by Henningsmoen 1953,p.243,271] Mississippian of Illinois.

SARSIELLA [S. capsula] Norman 1869,p.293 [Cytheridae by Norman; Sarsiellidae by Brady and Norman 1896, p.675] Recent, off British Isles.

SARSIELLIDAE Brady and Norman $1896, p .675$, a family of Myodocopa for the genera Sarsiella, Eurypy?us, and Nematohamma. 
SARSIELLINAE G. W. Müller 1906b,p.30, a subfamily of Cypridinidae for Sarsiella.

SARSOCYTHERE [S. patuxiensis] Tressler and Smith 1948,p.16 [Cytheridae-Cytherinae by TES] Recent, Maryland.

SAVAGELLA [Kirkbya lindahli Ulrich [1890] 1891,p.207,pl.18, [.6a-c] Geis 1932,p.168 [Kirkbyidae by Geis] Mississippian of Illinois. [not Savage]la Foerste 1920, Ohio J.Sci.,Echin., fide Neave, 1939,vol.4, p.118].

SCABERINA [S. nodomarginata] Bradfield 1935,p.67 [Kirkbyidae by Bradfield; : Roundyella by Cooper 1946,p.108] Pennsylvanian of Oklahoma.

SCABRICULOCYPRIS [S. trapezoides] Anderson 1940,p.377 [apparently Cytheridae from comparisons made by Anderson] Jurassic, England. [fresh-water].

SCHIZOCYTHERE [S. hollandica] Triebel 1950c,p.320 [Cytheridae-Cytherinae by Triebel] Miocene, Netherlands.

SCHLEROCHYLUS Sohn 1951,p.65 = Sclerochilus Sars 1865. SCHMIDTELLA [S. crassimarginata] Ulrich 1892,p.269 [Leperditidae by Ulrich; Leperditellidae by Bassler and Kellett 1934,p.471; Aparchitidae by Schmidt 1941, p.18,20,70; Leperditellidae-?Eridoconchinae by Henningsmoen 1953,p.251,256,275\} Ordovician, Wisconsin. 
SCHULERIDEA [S. acuminata] Swartz and Swain 1946,p.363-366 [Cytheridae-Cytherideinae by SES] Jurassic from well in Louisiana.

SCHWEYERINA [S. ovata] Zaspelova 1952,p.199 [Drepanellidae-Nodellinae by Zaspelova] Devonian, USSR.

SCLEROCHILUS [Cythere contorta Norman 1862a,p.48,pl.2,f.15]

Sars 1868,p.89 [Cytheridae-Paradoxostominae by Sars 1928,p.246] Recent, North Atlantic.

SCLEROCONCHA [Philomedes (Scleroconcha) appeloffi] Skogsberg $1920, p .419$ [as subgenus of Philomedes by Skogsberg] Recent, South Georgia.

SCLEROCYPRIS [S. clavularis] Sars 1924a,p.131 iCypridae by Sars] raised from dried South African mud.

SCOFIELDIA [Drepanella bilateralis Ulrich 1894,p.671,pl.46, f.35-38] Ulrich and Bassler 1908,p.314 [2ygobolbidae-Drepanellinae by Bassler and Kellett 1934, p.25; Primitiidae-Bolliinae by Kay 19402,p.261; Drepanellidae-Drepanellinae by Schmidt 1941,p.50] Ordovician, Minnesota.

SCOTTIA [Cypris browniana Jones 1850a,p.25,pl.3,f.la-d] Brady and Norman 1889,p.72 [Cypridae-Cyprinae by G. W. Müller 1912, p.219] Plejstocene, British Isles. 
SCOTTINI Bronstein 1947,p.152-154, a Tribe of Cypridae-Cyprinae for the genus Scottia.

SCROBICULA [Cytherella scrobiculata Jones, Kirkby and Brady 1884,p.76,pl.6,f.10] Posner 1951,p.53 [Scrobiculidae by Posner 1951, and by Polenova 1952,p.119] fide Polenova.

SCROBICULIDAE Posner 1951, fide Polenova 1952,p.120, who gives range as Devonian to Carboniferous.

SELEBRATINA, used as a subgenus of Gravia by Polenova 1952, p.82,84,143, author not indicated.

SELLULA [S. fallax] Wiman 1905,p.48 [ = Conchostraca by Ulrich and Bassler 1931,p.49; = a genus of Cambrian branchiopods by Bassler and Kellett 1934, p.472] Silurian, North Baltic.

SEMILUKIELLA, ? discussed by Polenova 1952,p.107 under Kloedenellidae-Cavellininae, author of genus not given.

SEMINOLITES [S. truncatus] Coryell 1928a,p.88 [Bairdiidae by Coryell; Thlipsuridae by Harlton 1929b,p.265; Healdiidae by Henningsmoen 1953,p.260] Pennsylvanian of Oklahoma.

SENESCELIA [S. marginata] Stewart and Hendrix 1945b,p.111 [Barychilinidae by SEH] Devonian, Ohio. 
SHIDELERITES [S. typus] Morris and Hill 1951,p.698 [Bairdiidae by Betty Kellett Nadeau in footnote at end of Morris and Hill's paper,p.699J Silurian, Indiana.

SIGMOBOLBINA [Entomis oblonga kuckersiana Bonnema 1909, p.67,pl.5,f.6-8 (not fig.9)] Henningsmoen 1953, p.206-208 [Sigmoopsiddae-Sigmoopsiinae by Henningsmoen] Ordovician drift, Germany.

SIGMOOPSIIDAE, Henningsmoen 1953,p.201,266, a family of Beyrichiacea, with subfamilies Sigmoopsinae and Glossopsiinae.

SIGMOOPSIINAE. Henningsmoen $1953, \mathrm{p} .203,266$, a subfamily of Sigmoopsidac to include Sigmoopsis, Ogmoopsis, Sigmobolbina, Carinobolbina, Bolbina, ?Kiesowia, ?Ctenobolbina, and Winchellatia.

SiGMopais [Cerat.npsis platyceras Öpik 1937,p.99,pl.2,f.6,7] Henningsmoen 1953,p.204,266 [Sigmoopsidae-Sigmnopsiznae by Henningsmoenj Ordovician, Estonia.

3byNIS [S. dictyotus] Kesling 1953c,p.4 [Aechminidae by. Kesling] Devonian, Ontario.

SILENITES [3. silenus] Coryel1 and Booth 1933,p.265 [Bairdiddae by C\&B; Cytherellidae by Kellett 1935,p.150, but Bairdiidae by Kellett 1935,p.166 in note at and of paper] Pennsylvanian, Texas. [Silenites 
(error pro Selenites Fischer 1877) Ancey 1880, fide Neave 1939, vol.4,p.194].

SIMPLICES Jones $1855 \mathrm{a}, \mathrm{P} .85$, a Group of species of the genus Beyrichia.

SINUSELLA [S. ignota] Spizharsky 1939,p.195 [Kirkbijidae (=Kirkbyidae) by Spizharsky; attributed in error to Lutkevich by Agnew 1944,p.219] Permian, USSR. Spizharsky indicates after the name: "in litt." so this may not be the original reference.

SIPHLOCANDONA [S. similis (Baird) (Candona similis Baird $1846, \mathrm{p} .415, \mathrm{pl} .9, \mathrm{f} .41$, and S. nomani, n.sp.] Brady 1910,p.210 [Cypridae-Candoninae by Brady] Recent, England.

SIPHONOSTRA [Cypridina (Siphonostra) spinifera] Skogsberg 1920,p.298 [as subgenus of Cypridina by Skogsberg] Recent, off Australia.

SPHAEROMICOLA [S. topsenti] Paris 1916,p.307-309 [Cytheridae-Entocytherinae by Hoff $1942 b, 2.65]$ Commensal on gills of Isopods and Amphipods, Europe.

SPHENICIBYSIS [S. hypoderota ] Kesling 1952a,p.24 [Leperditellidae by Kesling; ?Aparchitidae by Henningsmoen 1953,p.268] Devonian, Michigan. 
SPINOBAIRDIA [S. kellettae] Morris and Hill 1952,p.11

[Bairdiidae by MEH] Silurian, Tennessee.

SPINOVIA [3. distributa] Coryell and Malkin 1936,p.17

[Cytherellidae by CGM; Quasillitidae-Quasil]itinae by Henningsmoen 1953,p.257-275.] Devonian, Ontario.

SPIROCYPRIS [S. passiaca] Sharpe 1903,p.981 [ = Strandesia by Müller 1912,p.186; = Cypricercus by Blake 1933, p.230.] Recent, New Jersey.

SPONGICYTHERE [S. spissa] Howe 1951b,p.18 [Cytheridae-Cytherinae by Howe] Eocene, Florida.

STARINGIA Veen 1936,p.171, new name for Terquemia Veen 1932, non Tate 1868 [Cytherellidae by Veen; genotype not designated] Cretaceous, Limburg.

STENOCYPRIA [Cypris fischeri Lilljeborg, 1883,p.146, n. name for Cypris fasciata Fischer 1854,p.151,pl.5,f.9-12; pl.6,f.1,2; pl.11,f.9] G. W. Müller 190lb,p.571 [Cypridae-Cyprinae by G. W. Müller 1912,p.195] Recent, Europe.

STENOCYPRIS [Cypris cylindrica major Baird 1859,p.233, pl.63, $f .4=$ Cypris malcolmsoni Brady $1886 \mathrm{a}, \mathrm{p} .297, \mathrm{pl} .36$, g.5-7, new name as not Cypris cylindrica Sowerby $1840=$ Stenocypris malcolmsoni (Brady) of Sars $1889 a, p \cdot 28, p l \cdot 1, f \cdot 7,8 ; p l \cdot 5, f .1-4]$ Sars 1889a,p.27 
$[$ = subgenus of Cypris by vávra 1895,p.10; as a genus: Cypridae-Cyprinae by G. W. Müller 1912 , p.196] Recent, India.

STEPHENSONARIA [new name for Pseudocythereis Jennings 1936, not Pseudocythereis Skogsberg 1928] attributed to Corye11, by Stephenson 1946a,p.305. [ Anticythereis Van den Bold 1946,p.30, by Stephenson 1947b,p.579. Bold's paper appeared in print a few weeks earlier than Stephenson's.]

STEUSLOFFIA [Strepula linnarssoni Krause 1889a,p.16,pl.2, f.4,5, which a Beyrichia costata Linnarsson 1869 , p.85,pl.2,f.67-68, by Thorslund 1940,p.176] Ulrich and Bassler 1908,p.295; 1923,p.308 [as a subgenus of Beyrichia by UGB 1908; Zygobolbidae-Kloedeninae by Bassler and Kellett 1934,p.25, but Beyrichildae by Bassler and Kellett 1934,p.474; Piretellidae by Öpik 1937,p.110; ?repanellidae-w Bassleratiinae by Schmidt 1941,p.50; Hollinidae-Tetradellinae by Hessland 1949,p.352; Tetradellidae.Bassleratiinae by Henningsmoen 1953,p.217,220,223, 267) Silurian, Gotland.

STEUSLOFFINA [S. ulrichi] Teichert 1937a,p.120 [Bairdidae by Teichert; ?Beecherellidae by Agnew 1942,p.760] Ordovician, Arctic Canada. 
3TIBUS [S. kothornostibus] Swartz and Swain 1941,p.436 [ThI ipsuridae by SES] Devonian, Pennsylvania.

STRANDESIA [speeies not named] Stuhlmann 1888,p.1260, Recent, Zanzibar.

STRANDESIA iCypris (Stxandesia) mercatorum) vávra 1895, p.I8 las a subgenus of Cypris by Vávra; as genus: Cypridae-Cyprinae by G. W. Müller 1912,p.186] Recent, Zanzibar.

STREPTOLEBERIS \{S. Crenulata) Brady $1890,0.515 i=$ male Sarsielia by G. W. Müller 1912,p.36) Recent, off New CaIedonja.

STREPULA [3. concentrica] Jones and Holl $1886 \mathrm{~b}, \mathrm{p} .403$

[Beyrichidae by Ulrich 1894,p.632; Kirkbyidae by Bassler and Kellett 1934,p.476; Piretellidae by Opik 1937,p.111; Tetradellidas-Bassleratiinae by Henningsmoen 1953,p.220,245,267) Silurian, England.

STREPULITES [S. mooki] Coryel] and Malkin 1936,p.5

[Thlipsuridae by CGM] Devonian, Ontario.

SUBLIGACUhUM [s, scrobiculatum] Kesting and McMillan 1951, p.65 [holiinidae by KGM] Devonian, Michigan.

SUBTLiLA !S. prima) Zaspelova 1952,p.195 (Drepanellidae-. Nodellinae by Zaspeloya] Devonian, USSR. 
SUCHONELLA [S. typica] Spizharsky 1937,p.159 [Incertae Sedis by Agnew 1942,p.760] Permian, USSR. [Not indicated as new in this publication, but no reference given to previous paper. Cypridae by Spizharsky 1939,p.193]

SUCHONELIINA [S. cf. inornata [(M'Coy) Jones Cythere (?Cytherella) inornata M'Coy, Jones $1850 \mathrm{~b}, \mathrm{p} .63$, p1.18,f.9; S.(?) yanichevski, n.sp.] Spizharsky 1937,p.156. [Incertae Sedis by Agnew 1942,p.760] Permian, USSR. (Kuznetsk Basin) [Type not indicated, and genus not indicated as new, but no previous reference given. Cypridae by Spizharsky 1939, p.194]

SULCATIA, indicated as a subgenus of Gravia [Acronotellidae-Gravinae] by Polenova 1952,p.82, but author not given.

SULCELLA [S. sulcata] Coryell and Sample 1932,p.274

[Cytherellidae by CES; ?Healdiidae by Henningsmoen 1953,p.260] Pennsylvanian of Texas.

SULCICUNEUS [S, porrectinatium] Kesling 1951,p.222 [Primitiopsidae by Kesling; ?Hollinidae by Henningsmoen 1953,p.230,240,270] Devonian, Michigan.

SULCOCAVELLINA, mentioned under Kloedenellidae-Cavellininae by Polenova 1952,p.107, but author not given. 
SULCUNA [S. lepus, S. cuniculus, Jones $1873 \mathrm{~b}, p .411$, nude names; Jones and Kirkby $1874,0.37, \mathrm{pl} .411$ Jones 1873b,p.411, generic description [Cypridinidae by Bassler and Kellett 1934,p.478, who desipnate S. lepus genotype] Carboniferous, Ireland.

SVANTOVITES [S. primus] Pokorný 1950,p.539,601 [Glyptopleuridae by Pokorný; Kloedenellidae-Glyptopleurinae by Henningsmoen 1953,p.272] Devonian, Crechoslovakia.

SVAROGITES [S. spinosus] Pribyl [1951] 1953,p.24 [?Kirkbyidae by Pribylil Devonian, Bohemia.

SYNAPHE [Kirkbya annectens Jones and Kirkby 1866,p.42] Jones and K1rkby 1898,p.190 non Huebner 1825; non Thomson, $1864=$ Kirkby1a nom.nov., by Cossmann $1899, \mathrm{p} .45 ;$ Beyrichiella JGK, 1886a, by Ulrich and Bassler 1908,p.322] Carboniferous, Ireland.

SYNOPSIDA Daday 1900,p.124,126,207,215,304,305, a subtribe of Cyprinae for Cypris, Eucypris, Herpetocypris, Cyprois, Cypricercus; also as a subtribe of Candoninae for Cyclocypris, Cypria, Iliocypris, Ilyocyprella, Candonopsis, Eucandona, and Candona. 
TALIINNELIA [T. dimorpha] Öpik 1937,p.88 [Beyrichiidae by Öpik; Tetradellidae by Agnew 1942,p.760; Hollinidae-Tetradellinae by Schmidt 1941,p.37; Tetradellidae-Tetradellinae by Henningsmoen 1953,p.213,214,267] Ordovician, Estonia.

TANNELIA [T. gracilis] Kingma 1948,p.87 [Cytheridae-Cytherinae by Kingma] Pliocene, Sumatra.

TEICHOCHILINA [Isochilina jonesi Wetherby 1881,p.80,pl.2, f.7,7a] Swartz 1949,p.324 [Leperditiidae-Isochilininae by Swartz] Ordovician, Kentucky.

TERQUEMIA [T. falcoburgensis, T. convexa, T. limburgensis, T. longa] Veen 1932,p.345 [Cytherellidae by Veen; non Terquemia Tate, 1868, Lamell. = Staringia Veen 1936b,p.171] Cretaceous, Limburg.

TETRACORNELLA [T. ornata Zaspelova 1952,p.169,pl.4,f.I] Zaspelova 1952,p.168 [Drepanellidae-Neodrepanellinae by Zaspelova] Devonian, USSR.

TETRACYTHERURA [Cytheridea angulosa Seguenza 1880,p.363, pl.17,f.47] Ruggieri 1952b,p.86 [Cytheridae-Cytherurinae] Pliocene, Italy. 
TETRADELLA [Beyrichia quadrilirata Hall and Whitfield 1875 , p.105,pl.4,f.6,7] Ulrich 1890,p.112 [Beyrichiidae by Ulrich; Tetradellidae by Swartz 1936,p.551; Beyrichiidae-Tetradellinae by Kay 1940a,p.265; Hollinidae-Tetradellinae by Hessland $1948, p .98$, 99,340; Tetradellidae-Tetradellinae by Henningsmoen 1953,p.213,266] Ordovician, Ohio.

TETRADELIIDAE Swartz 1936,p.551, a family to include Dilobella, Tetradella, Ceratopsis, Kiesowia, Thomasatia, Bassleratia, Bellornatia, Raymondatia.

TETRADELIIDAE of Henningsmoen 1953,p.197,212,266 includes the subfamilies Tetradellinae, Piretellinae, and Bassleratiinae.

TETRADELLINAE Schmidt 1941,p.37 [ Tetradellidae Swartz 1936, (part); † Beyrichildae (part) + Piretellidae Opik 1937 (part) + Dilobellinae Kay 1940], a subfamily of Hollinidae for the genera: Tetradella, Tallinella, Ceratopsis, Kiesowia, Rigidella, Ctenobolbina (Ctenobolbina) and more doubtfully: Dilobella, Pseudostrepula, Ctenobolbina (Duhmbergia), Polyceratella.

TETRADELLINAF, Henningsmoen 1953,p.266, a subfamily of Tetradellidae for the genera Tetradella, Dilobella, Tallinnella, Hesslandella, Ceratopsis, ?Polyceratella. 
TETRAGONODON [Bradycinetus ctenorhynchus Brady 1881-1886, Fonds, IV, p.199, p.12,f.3-5; ?= Tetragonodon ctenorhynchus Brady \& Norman n.sp.1896. Also Tetragonodon erinaceus Brady and Norman; genotype not indicated] Brady and Norman 1896,p.667 [Cypridinidae by BGN; = Philomedes by G. W. Müller 1912,p.33; Genus good, but possibly as subgenus of Philomedes by Skogsberg 1920,p.348] Recent off Morocco.

TETRASACCULUS [T. bilobatus] Stewart 1936,p.744 [Primitiidae by Stewart; Hollinidae by Henningsmoen 1953, p.270] Devonian, Ohio.

TETRASTORTHYNX [T. diabolicus] Kesling 1953a,p.195 [Drepanellidae by Kesling] Devonian, Ontario.

TETRASULCATA [T. fluens] Matern 1929,p.40 [Beyrichiidae by Matern; Kloedenellidae? by Swartz 1936,p.549; Beyrichiacea of uncertain family by Henningsmoen 1953,p.274] Devonian, Germany.

TETRATYLUS [T. ellipticus] Cooper 1941,p.34 [Bairdildae by Cooper; ?Healdiidae by Henningsmoen 1953,p.260] Mississippian of Illinois.

THALMANNIA [T. sumatrensis] LeRoy 1939,p.272 [Cytheridae by LeRoy; Cytheridae-Cytherinae by Van den Bold 1946, p.32] Miocene, Sumatra. 
THAMINOCYPRIS [T. delicata] Zálanyi 1944,p.49,165 [Cypridae-Candoninae by Zálanyi] Neogene, Hungary.

THAUMATOCYPRINAE G. W. Müller $1906 \mathrm{a}, \mathrm{p} .41$, a subfamily of Halocypridae for the genus Thaumatocypris.

THAUMATOCYPRIS [T. echinata] G. W. Müller 1906a,p.41

[Halocypridae-Thaumatocyprinae by Müller] Recent, Indian Ocean.

THERIOSYNOECUM [Morrisonia wyomingensis Branson 1935,p.521, p1.57,f.17-21] Branson 1936,p.323 [new name for Morrisonia Branson 1935,p.521, not Morrisonia Grote 1874, a genus of living Lepidoptera; family not given by Branson, but appears to be Cytheridae-Limnocytherinae] Jurassic? Morrison formation, Wyoming.

THLIPSURA $[T$. corpulenta, T.V-scripta, T. tuberosa, the first designated genotype by Ulrich and Bassler 1923, p.317] Jones and Hol1 1869,p.213 [Thlipsuridae by Ulrich 1894,p.632] Silurian, England.

THLIPSURELLA IT. ellipsocleft,a] Swart, 1932,p.43[ThIipsuridae by Swartz] Devonian of Pennsylvania.

THLIPSURIDAE U1rich 1894,p.63\%, a family to include Thlipsura, Phreatura, and Octonaria. 
THL.IPSURIDAE of Ulrich and Bassler $1923, \mathrm{p} \cdot 316$, is placed in the Cypridacea with the following genera:

\section{Thlipsura, Octonaria, Phreatura, Craterellina.}

THLIPSURIDAE of Henningsmoen 1953,p.260,261, which he considers related to the Quasillitidae, Healdiidae, and possibly the Bairdidae, with the genera Fucraterellina, Eustephanella, ?Favullela, Hyphasmaphora, Octonaria, Oetonariella, ?Ranapeltis, Rothella, ?Stibus, Strepulites, Thlipsura, Thlipsurella, Thlipsurina, Thlipsuroides.

THLIPSURINA [T. elongata] Bassler 1941,p.26 [Thlipsuridae by Bassler; Kloedenellidae by Swain 1953,p.276] Devonian, Tennessee.

THLIPSURINA [Thlipsura tetragona Krause 1891,p.508,pl.32, f.15] Kummerow 1953,p.49 [Thlipsuridae by Kummerow; not Thlipsurina Bassler 1941] Silurian drift, Germany •

THLIPSUROIDES [T. thlipsuroides] Morris and Hill 1952,p.10 [Thlipsuridae by MEH] Silurian, Tennessee.

THOMASATIA [T. falcicosta] Kay 1934,p.337 [Zygobolbidae by Kay; Tetradellidae by Swartz 1936,p.551; Beyrichildae-Piretellinae by Kay 1940a,p.266; Drepanellidae-Bassleratiinae by Schmidt 1941, 50; Tetradellidae by Agnew 1942,p.760; Tetradellidae-Bassleratiinae by Henningsmoen 1953,p.267] Ordovician, Ontario. 
THRALIELLA [T. phaseolina] Stewart and Hendrix 1945b,p.111 [Cytherellidae by SGH] Devonian, Ohio.

TMEMOLOPHUS [T. margarotus] KesIing $1953 \mathrm{a}, \mathrm{p} .194$ [Drepanellidae by Keslingl Devonian of Ontario.

TOMIELLA [T. yavorskii] Spizharsky 1937,p.143-146 (Russian), 166 (English) [Spizharsky does not indicate family but includes 2 species formerly referred to Kirkbya; = Incertae Sedis by Agnew 1942,p.760] Permian of Kuznetsk Basin, USSR. [Primitidae by Spizharsky 1939,p.196].

TOULMINIA [T. hyalokystis] Munsey 1953,p.6 [Cytheridae-Cytherinae by Munsey] Paleocene, Alabama. [Not Toulminia Zittel 1878, spong., fide Neave 1939, $\operatorname{vol.4,p.512].~}$

TRACHYLEBERIDAE Sylvester-Bradley 1948d,p.793, a family of Cytheracea, for the following genera: Trachyleberis, Cythereis, Hemicythere, Pseudorythereis Skogsberg, Procythereis Skogsberg, Pterygocythereis, Buntonia, Eavella, Eucythereis, Isocythereis, Platycythereis, Anticythereis, and oligocythereis.

TRACHYLEBERINAE Hornibrook 1952,p.32-48, a subfamily of Cytheridae, in which Hornibrook includes Trachyleberis, Cythereis, Bradleya, Luadracythere, and Hemicythere. 
TRACHYLEBERIS [Cythere scabrocuneata Brady 1880 (not all figures)] Brady 1898b,p.444 [Cytheridae by Brady; = Cythereis by G. W. Müller 1912, p.336; Trachyleberidae by Sylvester-Bradley 1948d,p.793; Cytheridae-Trachyleberinae by Hornibrook 1942, p.32] Recent, Inland Sea of Japan. One should consult Harding and Sylvester-Bradley 1953 for their revision of this genus.

TREPOSELLA [Beyrichia lyoni Ulrich 1891,p.190,pl.14,f.2a-c, 3] Ulrich and Bassler 1908,p.314 [Beyrichiidae by Rassler and Kellett 1934,p.487; Beyrichildae-Beyrichilinae by Henningsmoen 1953,p.238,269] Devonjan, Kentucky.

TRIBOLBINA [T. carnegei] Latham 1932,p.358 [Beyrichiidae by Latham] Carboniferous, Scotland.

TRICERATINA [T. wrefordensis] Upson 1933,p.29 [Primitildae by Upson; = Monoceratina by Bassler and Kellett 1934,p.488; re-established by Cooper 1941,p.23 and placed in Acronotellidae] Permian of Nebraska.

TRICORNINA [T. navicula] Bouček 1936,p.50 [Primitiidae-Primitilnae by Bouček; ?Acronotellidae by Agnew 1942, Alanellidae by Henningsmoen 1953,p.235,247, 273] Silurian, Bohemia. 
TRIEBELINA [T. indopacifica] Bold 1946,p.73,74 [Bairdiidae by Bold] Recent, Malakka Straits.

TRUBINELLA [Hippa latens Barrande 1872,p.517,pl.26,f.3a-d] Pribyl 1951,p.102, new name for Hippa Barrande 1872, not Fabricius 1787. Ordovician, Bohemia.

TRYPETERA [T. barathrota] Kesling 1954,p.178 [Barychilinidae by Kesling] Devonian, Ohio.

TUBEROCYPRIS [T. acuminatus] Swain 1947,p.522 [Cypridae, near Candona by Swain] Late Tertiary of Utah.

TUBEROCYPROIDES [T. dipleura] Swain 1947,p.525 [Cypridae by Swain] Late Tertiary of Utah.

TUBLIBAIRDIA [T. tubulifera] Swartz 1936,p.58] [Bairdiidae by Swartz; = Pachydomella by Swain 1953,p.280] Devonian, Pennsylvania.

TYPHLOCYPRIS [Cypris eremita V́ejdovský 1880,p.XLIX-LVI] V́ejdovský 1882,p.64 [as a subgenus of cypris by Vejdovský; = Candona by G. W. Muller 1912,p.144, with the concept that Candona was based on $\underline{\text {. }}$ candida (O.F.Müller) rather than C. reptans as designated by Baird 1846,p.414; Typhlocypris therefore appears valid as a genus, and is probably the first generic name available for the large $\underline{C}$. candida group of species] Recent, Europe. 
TYPHLOPSIDA Daday 1900,p.271, a Tribe of CypridaeCandoninae for the genus Typhlocypris.

UHAKIELLA [U. coelodesma] Öpik 1937,p.106 [Beyrichiidae by Henningsmoen 1953,p.226,267; Primitiidae but probably synonym of Primitia by Henningsmoen 1953, p.226,267] Ordovician, Estonia.

ULLERELlA [Ullia ulli Dons, in Dons and Henningsmoen 1949, p.28,pl.1,f.1-4] Henningsmoen 1950,p.244, new name for Ullia Henningsmoen 1949, not Roewer 1943 [Beyrichiacean ostracods related to Steusloffia by Dons and Henningsmoen; Beyrichiacea of unknown family by Henningsmoen.1953,p.274] Ordovician, Norway.

ULLIA [U. ulli Dons] Henningsmoen, in Dons and Henningsmoen 1949,p.27 [not Ullia Roewer 1943; = Ullerella, nom. nov., by Henningsmoen 1950,p.244] Ordovician, Norway •

ULRICHELLA [U. remesi] Bouček 1936,p.73 [Beecherellidae by Bouček; may not be an ostracod by Agnew 1942 , p.760] Silurian, Bohemia.

ULRICHIA [U. conradi] Jones 1890b,p.543 [Beyrichiidae by Ulrich 1894,p.632; Primit1idae by Bassler and Kellett 1934,p.488; Primitiidae-Primitinae by Bouček 1936,p.46; Drepanellidae-Ulrichilnae by 
Schmidt 1941,p.51; Drepane11idae-?Bollinae by Henningsmoen 1953,p.233,268] Devonian, Thedford, Canada.

ULRICHIINAE Schmidt 1941,p.51, a subfamily of Drepanellidae, for the genera U1richia, Pseudulrichia, Pyxiprimitia, ?Jonezites, Hal1ielia, ?Hallatia. [This subfamily = Bolli inae Bouček, 1936, by Henningsmoen $1953, p \cdot 197,233,268]$.

ULWELLIA [U. menevensis] Anderson 1939,p.300 [Cypridae-. Rostocyprinae by Anderson; = subgenus of Cypridea. by Sylvester-Bradley 1949,p.126;as a genus:

Cypridae-Cyprideinae by Peck 1951,p.320] Jurassic, England.

UNICORNITES [U. homeomorphus] Pokorný 1950,p.560,561

[Podocopa, no certain family by Pokorný] Devonian, Czechoslovakia.

UROCYTHERE [U. attenuata] Howe 1951b,p.17 [Cytheridae-Cytherinae by Howe] Eocene, Florida.

UROCYTHEREIS [Cytherina favosa Roemer 1838,p.516,pl.6,f.7] Ruggieri 1950a,p.28 [Cytheridae] Pliocene, Italy.

\section{V}

VARGULA [Cypridina norvegica Baird 1860,p.200,pl.71,f.4,4a-d] Skogsberg $1920, p .245$ [as a subgenus of Cypridina by Skogsberg] Recent off Norway. 
VARICOBAIRDIA [Bairdia (Varicobairdia) kettneri] Pokorný $1950, p \cdot 546,609$ [as subgenus of Bairdia by Pokorný] Devonian, Czechoslovakia.

VARIX [V. propria] Cooper 1947,p.90 [Drepanellidae by Cooper; ?Kloedenellidae-Glyptopleurinae by Henningsmoen 1953,p.272] Mississippian of Illinois.

VENULA [Primitiopsis? striatus Croneis and Funkhouser 1938, p.340,pl.10,1.12] Cooper 1941,p.44 [Glyptopleuridae by Cooper; Kloedenellidae-Glyptopleurinae by Henningsmoen 1953,p.272] Mississippian of Illinois.

VERRUCOLSELLA Neave $1950, \mathrm{vo1} .5, \mathrm{p} .290=$ Verrucosella Croneis and Gale 1939.

VERRUCOSELLA [V. golcondensis] Croneis and Gale 1939,p.275 [ Cornigella Warthin, by Cooper 1941,p.39] Mississippian of Illinois.

VERTEXIA [V. tauricornis] Lutkevich 1939,p.192,pl.XLVI, fig.4 [described by Lutkevich as a phyllopod, but erroneously listed by Agnew 1944,p.219, and by Branson 1948,p.882, as an ostracod; Neave 1950, vol.5,p.90 gives the date erroneously as 1941] Permian, USSR.

VLTAVINA [V. bohemica] Bouček 1936,p.47 [Primitiidae-Primitiinae by Bouček; Acronotellidae by Agnew, 1942, p.760, who suggests may not be an ostracod; 
Alanellidae by Henningsmoen 1953,p.247,273]

Silurian, Bohemia.

VOGDESELLA [Melanella hemidiscus Wade 1911,p.451,f.9a,b]

Baker 1924,p.187,188, new name for Melanella wade, 1911, not Bory, 1824 [Drepanellidae-?Bolliinae by Henningsmoen 1953,p.269; = Jonesella Ulrich 1890, by Bassler and Kellett 1934,p.491] Ordovician, Wales.

VORONINA [V. voronensis] Polenova 1952,p.140 [Incertae Sedis by Polenova] Devonian, USSR.

W

WALDRONITES [Cornulina bispinosa Coryell and Williamson 1936, p.2,fig.3] Coryell and Williamson 1942, in Agnew $1942, p .760$, new name for Cornulina Coryell and Williamson 1936, not Conrad 1853, Moll.; Primitiidae by CEW; Aechminidae by Agnew 1942,p.760; Drepanellidae-?Aechmininae by Henningsmoen 1953, p.269] Silurian, Indiana.

WARTHINIA [Primitia nodosa Ulrich 1890,p.134,pl.10,f.1la-12] Spivey 1939,p.167 [?Beyrichiidae by Agnew 1942, p.760; Drepanellidae-?Bolliinae by Henningsmoen 1953,p.233,268] Ordovician, or Silurian, Cincinnati, Ohio. 
WAYLANDELLA [W. spinosa] Corye11 and Billings 1932,p.175

[Bairdildae by CGB] Pennsylvanian, Texas.

WELLERIA [W. obliqua] Ulrich and Bassler 1923a,p.307

[Zygobolbidae-Kloedeninae by Bassler and Kellett 1934,p.492; Beyrichiidae-Beyrichilnae by Henningsmoen 1953,p.238,269] Silurian, West Virginia.

WHIPPLELLA [W. cuneiformis] Holland 1934,p.343 [Aparchitidae by Holland; Primitildae by Agnew 1942, p.760; Cypridae by Scott $1944, \mathrm{p} \cdot 142=$ Carbonita by Cooper 1946,p.66] Permian, Pennsylvania.

WINCHELLATIA [W. longispina] Kay 1940a,p.253 [Primitiidae-Eurychilininae by Kay; Hollinidae-Ctenentominae by Schmidt 1941,p.34; Sigmoopsiidae-?Sigmoopsiinae by Henningsmoen 1953,p.207,212,236,266] Ordovician, Iowa.

WORKMANELLA [W. distincta] Croneis and Gale 1939,p.277 [Kirkbyidae by CEG; = Tetrasacculus by Cooper 1941,p.63] Mississippian of Illinois. XENOCYTHERE [Cythere cuneiformis Brady 1868b,p.404,pl.31, f.47-54] Sars 1925,p.179 [Cytheridae-Cytherinae by Triebel 1949,p.186] Recent, North Atlantic. 
XESTOLEBLRINAE Sars $1928,0.242$, a subfamily of Cytheridae to include Xestoleberis, Microxestoleberis, and Metacypris.

XESTOLEBtikIS ? 2 snecies: Cythere nitida Liljebora, and C. auriantia Baird 1838, vol.2.p.143,n1.5,f.26; the latter being designated tyne by Brady and Norman 1889,p.188] Sars 1866,p.66,67 [Cytheridae-Xestoleberinae by Sars $1928,0.242$ ] Recent, North At lantic, more than 100 described species.

XIPHICHILUS 12 species: Bythocytherf trisisima Nomar. $1869, p .294=$ Xiphichilus tenusissimus (Norman) by Brady; and $X$. amypdaloides,n.sn. I Brady 1870, n. 369 Tnot Xiphocheilus Bleeker 1856 Machaerina Brady and Norman 1889,p.237, nom.nov.! Recent. British Isles.

XYSTINOTUS 「X. wriphtorum; Keslinp 1953a,n.197 TDrenanelIldae by Keslingl Devonian, Ontario.

YOUNGIA :Y. roctidorsalisl Jones and Kirkby 1886c,p. 5017; Jones and Kirkby 1887,p.515! Cythoridae by Ulrich $1894,0.632$; not Youngia Lindstrom 1885, a Trilobite; Younginlla Jones and Kirkby 1895.0.456, nom.nov. 7 
YOUNGIELLA [Youngia rectidorsalis Jones and Kirkby 1886 , p. 515 = Youngiella rectidorsalis Jones and Kirkby $1895, \mathrm{p} \cdot 456, \mathrm{pl} .2 \mathrm{l}, \mathrm{fog} \cdot 5 \mathrm{a}-\mathrm{d}\rfloor$ Jones and Kirkby 1895, p.456, nom.nov., for Youngia [Youngiellidae by Kellett 1933,p.103] Carboniferous, England.

YOUNGIELLIDAE Kellett $1933, \mathrm{p} .103$, a family to include Youngiella and Moorites. Henningsmoen 1953, p.246, 273 adds: ?Moorea, and ?Hardinia.

Z

ZABOROVIA [Z. obscura] Polenova 1952,p.69 [Leperditellidae by Polenova] Devonian, USSR.

ZONOCYPRIS [z. madagascarensis, Z. elegans] G. W. Müller, $1898, p .284$ [Cyprididae-Cypridopsinae by Sharpe 1903,p.990; Cypridae-Cyprinae by G. W. Müller 1912,p.217] Recent, Madagascar.

ZONOZOE [z. drabcwiensis, Z. complexa] Barrande 1872,p.554, "Middle Silurian" Bohemia.

ZYGOBEYRICHIA [2. apicalis] Ulrich 1916,p.290 [Beyrichiidae by Ulrich; Zygobolbidae-Kloedeninae by Bassler and Kellett 1934,p.494; Beyrichiidae-Beyrichiinae by Henningsmoen 1953,p.238,269] Devonian, Maine. 
ZYGOBOLBA [Beyrichia decora Billings $1866, \mathrm{p} .67=z_{\text {yobolba }}$ decora (Billings) Ulrich and Bassler 1923,p.537, $\mathrm{pl} \cdot 39, f \cdot 15-22 ; \mathrm{pl} \cdot 40, f \cdot 11-14 ; \mathrm{pl} \cdot 64, f \cdot 21-25]$ Ulrich and Bassler 1923a,p.308 [Zygobolbidae-Kloedeninae by UEB; Beyrichiidae-Zygobolbinae by Henningsmoen 1953,p.269] Silurian, Anticosti.

ZYGOBOLBIDAE Ulrich and Bassler 1923a,p.304, a family of Beyrichiacea, composed of the subfamilies: Zygobolbinae, Drepanellinae, and Kloedeninae.

ZYGOBOLBINAE Ulrich and Bassler 1923a,p.304, a subfamily of Zygobolbidae to include: Zygobolba, Zygobolbina, Zygosella, and Bonnemaia.

ZYGOBOLBINAE of Henningsmoen 1953,p.238,269, a subfamily of Beyrichiidae to include: Zygobolba, Zygobolbina, Zygosella, Mastigobolbina, Plethobolbina, Bonnemaia, and ?craspedobolbina.

ZYGOBOLBINA [2. conradi] Ulrich and Bassler 1923a,p.305 [Zygobolbidae-Zygobolbinae by UEB; Beyrichildae-Zygobolbinae by Henningsmoen 1953,p.269] Silurian of New York.

ZYGOBOLBOIDES [Z, grafensis] Spivey 1939,p.169 [Zygobolbinae by Spivey; Zygobolbidae by Agnew 1942,p.761; Drepanellidae-?Bolliinae by Henningsmoen 1953, p.268] Ordovician, Iowa. 
ZYGOPSIDA Daday 1900, p. 124,125,207,209,304,305, a subtribe of Cypridae, used by Daday under the subfamily

Cyprinae for Centrocypris, and under the subfamily Candoninae for Notodromas.

ZYGOSELLA [Z, vallata] U1rich and Bassler 1923a,p.305

[Zygobolbidae-Zygobolbinae by UEB] S1lurian, West virginia. 
Agnew, Allen F. 1942. BIBLIOGRAPHIC INDEX OF NEW GENERA AND FAMILIES OF PALEOZOIC OSTRACODA SINCE 1934• Jour. Paleo., vol. 16, no. 6, p.756-763. [Boucia, n. name for Basslerella Bouček, 1936, non Kellett 1935; Waldronites Coryell and Williamson, n. name for Cornulina CEW, 1936, non Conrad 1853; Opikium, n. name for Biflabellum Öpik 1935, non Doederlein 1913]. Film L.S.U.

- 1944. ADDENDA AND ERRATA TO BIBLIOGRAPHY OF OSTRACODES. Jour. Paleo., vol. 18, no.2, p. 218-219. Film. I.S.U.

Alexander, Charles Ivan. 1929. OSTRACODA OF THE CRETACEOUS OF NORTH TEXAS. Texas University Bulletin 2907, 137pp., 10 pls., distribution chart. [Cytherelloidea n. gen.; $90 \mathrm{sp.}$, and 3 vars. of which $56 \mathrm{sp}$ and 1 var. are described as new.] Film L.S.U.

- 1933. SHELI. STRUCTURF, OF THE OSTRACODE GENUS CYTHEROPTERON, AND FOSSIL SPECIES FROM THE CRETACEOUS OF TEXAS. Jour. Paleo., vol. 7, no. 2, p.181-214, pls.25-27. [Cytheropteron defined from shell structure, with Cytheropteron, ss. and Eocytheropteron, new subgen.; Drthonotacythere, Brachycythere, n. gen•; $11 \mathrm{n}$. sp., and 2 new names. 1 Film L.S.U. 
- 1934. OSTRACODA OF THE GENERA MONOCERATINA AND ORTHONOTACYTHERE FROM THE CRETACEOUS OF TEXAS. Journ. Paleo., vol. 8, no. 1, p. 57-67, pl.8. [5 n.sp. of Monoceratina; 3 of Orthonotacythere. Suggests Monoceratina may be ancestor of Cytherurinae, Loxoconchinae, and Bythocytherinae. Film I:S.U.

- 1934 b. OSTRACODA OF THE MIDWAY (EOCENE) OF TEXAS. Journ. Paleo., vol. 8, no.2, p.206-237, pls. 32-35. [38 sp., 31 new, one of which, Cytherella excavata, p. 211, was made genotype of Morrowina, by Loetterle 1937]. Film I.S.U.

- 1936. OSTRACODA OF THE GENERA EUCYTHERE, CYTHERURA, EUCYTHERURA, AND LOXOCONCHA FROM THE CRETACEOUS OF TEXAS. Journ. Paleo., vol. 10, no. 8, p.639-694, p1. 93. [9 new species]. Film I.S.U.

Alm, Gunnar. 1915. MONOGRAPHIE DER SCHWEDISCHEN SÜSSWASSER-OSTRACODEN NEBST SYSTEMATISCHEN BESPRECHUNGEN DER TRIBUS PODOCOPA. Upsala, Universitet. Zool. Bidrag från Uppsala, vol. 4, P.1-247, 1 pl. [A reclassification of the Cypridae, using it in a superfamily sense with Gruppe Pontocypridae, Macrocypridae, and Eucypridae used essentially as genera: Ilyocyprinae, Cyprinae and Candocyprinae Macrocyprinae as subgenera, plus tribes. P. 160-237 devoted to the biology of northern fresh-water ostracoda]. Film U.III. 
Almeida, Fernando F.M. de. 1950. UMA FAUNULA DE CRUSTÁCEOS BIVALVOS DO ARENITO BOTUCATÚ NO ESTADO DE SÃO PAULO. Brazil. Min. Agricultura, Dept. Nac. Prod. Min., Div. Geol. Min, Boletim no. 134, 38pp., 4 pls. [Ostracods p.30-36,pl.4, and text figs 5-8.] [Pachecoia,

Candonopsis n. gen. and 3 n.sp.] Film L.S.U.

Ammon, I. V. 1910. in: Bavaria, K. Bayerische oberbergamt Geognostische Beschreibung des Königreichs Bayern. Erlauterung zu dem Blätte, Nr. X. München, 1910. [from Güthorl 1934,p.26] [This has not been located. It is not listed in the Union Catalog. The request was circularized in April, with no results so far.... Miss Karlson]. [Cuselina, n. gen.]

Anderson, F. W. 1939. WEALDEN AND PURBECK OSTRACODA. Ann. and Mag. Nat. Hist., Ser. 11, vol. 3, p. 29l-310, pls. 12, 13. [Rostocyprinae, n. subfam. of Cypridae; Ulwellia, Langtonia, Morinina, Cyamocypris, n. gen., and 10 n.sp.] Film U. Illinois.

1940. OSTRACODA FROM THE PORTLAND AND PURBECK BEDS AT SWINDON. Geologists Assoc., London, Proceedings, vol. 51, p. 373-384, pls. 18, 19. [16 n.sp. and vars.; Scabriculocypris, Rhinocypris, n. gen.] Film L.S.U. 
Armstrong, James. 1871. A GENERAL CATALOGUE OF THE FOSSILS AND THEIR MODE OF OCCURRENCE AND AN INDEX TO THE PRINCIPAL LOCALITIES, in ON THE CARBONIFEROUS FOSSILS OF THE WEST OF SCOTLAND, by John Young. Geol. Soc., Glasgow, Trans. vol. 3, Supplement, [Ostracods, p.2529; a list of 53 species; Cypridinopsis, nom.nud., p.26]. Film Univ.Minnesota.

Baird, William. 1835. LIST OF ENTOMOSTRACA FOUND IN BERWICKSHIRE. Berwickshire Naturalists Club [History] Proc., p.95-100,pl.3. [Cythere reinformis, c. alba, C. variabilis, Cypris joanna, C. minuta, $\underline{\text { c. elongata, }}$ C. reptans, $\underline{\text {. westwoodii, }}$. . hispida, $\underline{\text { C. lucens, }}$. compressa described as new species.] Film from Harvard. 1837-1838. THE NATURAL HISTORY OF THE BRITISH ENTOMOSTRACA. The Magazine of Zoology and Botany, Edinburgh vol.1,1837,p.35-41;309-333, 514-526,pls.8-10. Vol.2, $1838, \mathrm{p} .132-144 ; 400-412, \mathrm{pl} .16$ [Ostracoda 1837, p.514-526, anatomy of ostracods, and Cypris pubera, and C. detecta. 1838,p.132-144: C. strigata, C. vidua, C. monacha, $\underline{\text {. }}$ candida, $\underline{\text {. fuscata, }}$. hispida, $\underline{\text {. compressa, }}$ C. minuta, . joanna, $\underline{\text {. }}$. elongata, C. westwoodi.i, C. gibbosa, C. clavata, Cythere flavida, c. reniformis, ‥ albomaculata, ‥ alba, c. variabilis, C. aurantia, . nigrescens.] Continued in Annals of of Natural History. Film, University of Illinois. 
Baird, William. 1843. NOTES ON BRITISH ENTOMOSTRACA. Zoologist, a monthly journal of natural history, vol. 1,p.193-197. [Cythere inopinata Baird, p.195,figs.a-e]. Film, Univ. Illinois.

- 1845. ARRANGEMENT OF THE BRITISH ENTOMOSTRACA, WITH A LIST OF SPECIES, PARTICULARLY NOTICING THOSE WHICH HAVE AS YET BEEN DISCOVERED WITHIN THE BOUNDS OF THE CLUB. Berwickshire Naturalists Club [History] Proceedings, [1842-1849], p.145-148. [Cypris elliptica, C. Sella, nude names; Genus Candona, new, with $\underline{\text {. }}$.

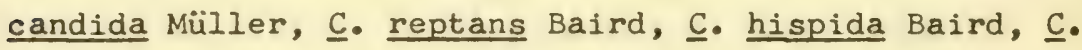
detecta Müller, and C. similis, n.sp. listed in order, the last nude. Family Cypridae, n.fam. for Cypria, Candona and Cythere]. Film from Harvard.

- 1846. DESCRIPTION OF SOME NEW GENERA AND SPECIES OF BRITISH ENTOMOSTRACA. Ann. Mag. Nat. Hist., ser.1,vol.17,p.410-416,pl.9. [Description of Candona Baird 1845 repeated, with Cypris reptans Baird 1835 designated as type' Cypris elliptica, $\underline{\text { C. sella, Candona }}$ similis described as new species.] Film, I.S.U.

- 1850a. THE NATURAL HISTORY OF THE BRITISH ENTOMOSTRACA, London, printed for the Ray Scoeity, pp. i-viii, 1-364, pls.1-36. [Ostracoda p.138-182, pls.18-23. Families Cytheridae, and Cypridinadae, new; 5 n.sp. of Cythere; 2 of Cythereis, and 1 of Cypridina. 


\section{- (Continued)}

The spelling of Cypridae, p.139, is corrected to

Cyprididae by Baird in the introduction, p.vili, fide Jones $1857, \mathrm{p} .6]$. Film, Lib. Congress. [minus introduction].

1859. DESCRIPTION OF SOME NEW RECENT ENTOMOSTRACA FROM NAGPUR, COLLECTED BY THE REV. S. HISLOP. 20ol. Soc. London, Proc., Pt.27,p.231-234,pl.63. [Cypris subglobosa, C. cylindrica and var. major, $\underline{\text { C. }}$ dentatomarginata, n. spp.]. Film, University of Illinois.

- 1860. NOTE UPON THE GENUS CYPRIDINA, MILNE-EDWARDS, WITH A DESCRIPTION OF SOME NEW SPECIES. ZOOI. Soc. London, Proc., vol. 28,p.199-202,plate 71

[Cypridina norvegica, $\underline{C}$. godehevi, $\underline{C}$. ovum, $\underline{C}$. albo-maculata]. Film, University of Illinois.

Baker, Fred. 1924. VOGDESELLA, A NEW GENUS-NAME FOR A PALEOZOIC CRUSTACEAN. California Acad. Sci. Proc. vol. 13, no., p. 187-188. Film, L.S.U.

Barrande, Joachim. 1872. SYSTÊME SILURIEN DU CENTRE DE

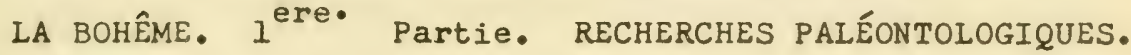
Supplement au Vol. 1. TRILOBITES, CRUSTACÉS DIVERS ET POISSONS. Troisiéme Partie. CRUSTACÉS DIVERS, NON TRILOBITIQUES, DES FAUNES SILURIENNES DE LA BOHEME. 
Barrande, Joachim. 1872. (Continued).

["Ostracodes," p.466-555, plates (published separately in atlas) nos. 22-27, 32, 34] Prague and Paris. [23 genera discussed; Aristozoe, Bolbozoe, Callizoe, Caryon, Crescentilla, Elpe, Hippa, Nothozoe, Orozoe, Zonozoe, n.gen. Several of Barrande's genera have been transferred to the Conchostraca by Ulrich and Bassler 1931, and thence to the order Bradorina by Raymond 1935, 1946]. Film, I.S.U.

Bassler, R. S. 1932. THE STRATIGRAPHY OF THE CENTRAL BASIN OF TENNESSEE. Tenn. State Geol. Surv., Bull. 38,p.1-268, 49 pls. Nashville. [Ostracods, p.146,194,224,236, pls. 6,21,27; Allostraca, Paracythere, n.gen.]. Film, L.S.U. 1952.

- 1941. OSTRACODA FROM THE DEVONIAN (ONONDAGA) CHERT OF WEST TENNESSEE. Washington Acad. Sci. Jour,, vol.31,no.1,p.21-27, and 37 figs. [Ctenoloculina, Thlipsurina, Ranapeltis, Octonariella, n.gen., and 24 n.sp.]. Film, L.S.U. and Kellett, Betty. 1934. BIBLIOGRAPHIC INDEX OF PALEOZOIC OSTRACODA. Geol. Soc. Amer., Special Paper No. 1, 500 pp., illus. LLeperditacea, new superfamily; Primitiinae, $n$. subfam. of Primitiidae Saffordellina, nom.nov.l. Film, I.S.U. 
Batalina, M. A. 1926. ON THE LOWER CARBONIFEROUS OSTRACODA FROM THE BOROVITCHI DISTRICT OF DEPARTMENT OF NOVGOROD. Russia, Geologicheskii Komitet, [Bulletins du Comité Géologique] vol. XIIII, No. 10 [1924] 1926, p.13151338,pls.22-23. [Hollina stepanovi, n.sp.; Janischewskya digitata, n.gen., n.sp.J. Film from Library of Congress.

Bean, William. 1836. DESCRIPTION AND FIGURES OF UNIO DISTORTUS BEAN, AND CYPRIS CONCENTRICA BEAN, FROM THE UPPER SANDSTONE AND SHALE OF SCARBOROUGH: CYPRIS ARCUATA BEAN FROM THE COAL FORMATION OF NEWCASTLE. Mag. Nat. Hist. vol.9,p.376,377, figs.53-55. London. Film, University of Illinois.

Bigsby, John J. 1868. THESAURUS SILURICUS. THE FLORA AND FAUNA OF THE SILURIAN PERIOD. WITH ADDENDA FROM RECENT ACQUISITIONS. London, John Van Voorst publisher, $214 \mathrm{pp}$. [Ostracods listed, p.72-77; 199-200. A number of species first mentioned here]. Film of USGS Copy made L.S.U., Feb. 1953.

Billings, E. 1866. CATALOGUES OF THE SILURIAN FOSSILS OF THE ISLAND OF ANTICOSTI, WITH DESCRIPTIONS OF SOME NEW GENERA AND SPECIES. Canada Geol. Survey, Montreal, 93pp., figs. [Beyrichia decora, B. venusta, n.spp., Leperditia anticostiana Jones,p.67,68]. Film of Smithsonian Institution copy. 
Blake, Chas. 1933. ORDER OSTRACODA. in Biological Survey of the Mount Desert Region conducted by William Proctor, Part V, p.229-241, figs. 39,40. Published by the Wistar Institute of Anatomy and Biology, Philadelphia. [Cushmanidea, n.gen., Pterygocythereis, n.subgen. of Cythereis, many species discussed, and key given to subfamilies of Cytheridae.] Film, U.S. Dept. Agriculture Library.

Bold, Willem Aaldert van den. 1946. CONTRIBUTION TO THE STUDY OF OSTRACODA WITH SPECIAL REFERENCE TO THE TERTIARY AND CRETACEOUS MICROFAUNA OF THE CARIBBEAN REGION. Amsterdam, July 11, 1946. 167pp., 18 pls., text figs. Published by J. H. DeBussy. [Triebelina, Ruttenella, n.gen; Anticythereis nom.nov•; over 100 new spacies, and extensive bibliography]. Film, L.S.U.

19502. HEMIKRITHE, A NEW GENUS OF OSTRACODA FROM THE INDOPACIFIC. Ann. Mag. Nat. Hist., ser. 12, vol. $3, p .900-904,4$ text figs. Film, L.S.U.

1950b. MIOCENE OSTRACODA FRCM VENEZUELA.

Journ. Paleo., vol. 24, no.1, 1950,p.76-88, pls. 18, 19, 4 figs. [31 species, 11 new; Javanella Kingma $=$ Pellucistoma Coryell and Fields]. Film, L.S.U.

Bo11, Ernst. 1847. BEITRAG ZUR KENNTNISS DER TRILOBITEN. Palaeontographica, vol.1, no.3, p.126, 127 [Beyrichia, n.gen.]. Film, L.S.U. 
Bonnema, J. H. 1909. BEITRAG ZUR KENNTNIS DER OSTRAKODEN DER KUCKERSSCHEN SCHICHT $\left(\mathrm{C}_{2}\right)$. Groningen, Rijksuniversiteit, Min.-Geol. Inst., Mitteilungen, $\mathrm{Bd} \cdot 2$, Heft.1, $84 \mathrm{pp} ., 8 \mathrm{pls}$. [29 new species and vars.]. Film of copy borrowed from Mt. Holyoke.

1933b. ÜBER PALÄOZOISCHE OSTRACODEN • Zeitschr. f. Geschiebeforschung, vol.9,p.150-156 [Cytherella troedssoni, n. name, Eocytherella, n.gen.]. Film,L.S.U. - 1938. ZUM ALLERLETZTEN MALE: DIE AUfSTELlung DER SCHALEN DER PÄLAOZOISCHEN OSTRACODEN UND DIE BRUTKAMMER VON PRIMITIOPSIS OBLONGA J \& H. Natuurhist. Maandbl. Jg.27, no.10, p.104-107, 19 figs. Limburg (Maastricht). [Chilobolba, sic., p.104]. Film, N.Y.St. Dept. Agr. Lib.

Bosquet, J. 1852. DESCRIPTION DES ENTOMOSTRACÉS FOSSILES DES TERRAINS TERTIARES DE LA FRANCE ET DE LA BELGIQUE. Acad. Roy: Sci., Lettres, Beaux-Arts de Belgique, MEm. couronnés et Mém Savants étrangers, vol. $24,142 \mathrm{pp}$. 6 pls. [2 n.sp. Cytherella; 4 of Bairdia, 3 of Cytheridea; 38 of Cythere; 1 of Cyprella. Cypridea and Cytheridea, n.gen.]. Film, Crear Library.

Bouček, Bedřich. 1936. DIE OSTRACODEN DES BÖHMISCHEN LUDLOWS (STUFE e $\beta$ ). Neues Jahrb., Beil.-Bd.76, Abt. B, Heft.1,F.31-98, pls.206. [Bolbozoidae, Alanellidae, 
new families; Bolliinae Primitiinae, Aechmininae, new subfamilies; Ectoprimitia, Vltavina, Tricornina, Mirochilina, Novakina, Basslerella, Berounella, Karlsteinella. Budnianella. Alanella, Ulrichella, new genera: Neoaparchites,.n. subgen.]. Film, L.S.U.

1937. ÜBER EINIGE OSTRAKODEN AUS DER STUFE $\mathbf{e}_{\mathbf{a}}$ DES BÖHMISCHEN SILURS. (SUR LA TROUVAILLE DES OSTRACODES DANS LA BANDE a DUR SILURIEN DE LA BOHÉME). Česká Společnost Nauk, Prague, Věstnik, An. 1936, vol. 2, $11 \mathrm{pp} ., 5 \mathrm{figs}$. [Daleiella, n.gen., and $4 \mathrm{n.sp.}$. Film, L.S.U.

Bradfield, Herbert Henry. 1935. PENNSYLVANIAN OSTRACODS OF THE ARDMORE BASIN, OKLAHOMA. BuIl. Amer. Paleontology, vol. 22, no. $73,145 \mathrm{pp}, 13 \mathrm{pls}$. [New genera: CoryelIina, Mammoides, Nuferella, Roundyella, Scaberina, Bairdiacynris, Healdiacypris, Harltonella, Cavellinella, Armorea, Binodella and 93 new species and varieties]. Film, I.S.U.

Brady, G. S. 1865. ON UNDESCRIBED FOSSIL ENTOMOSTRACA FROM THE BRICK-EARTH OF THE NAR. Ann. Mag. Nat. Hist., ser. 3, vol. 16, p.189-191, pl.9. [Cytheridea puncti11ata, Cythere carinata, $\underline{\text { c. arborescens, }}$ c. aspera, n. sp. In a footnote Normania mentioned in Jones 1865 is abandoned]. Film, University of Wisconsin. 
- 1866a. ON NEW OR IMPERFECTLY KNOWN SPECIES OF

MARINE OSTRACODA. Zool. Soc. London, Trans., vol.5, p.359-393, pls. 57-62. [Jonesia, Normania, Heterodesmus, n.gen., and 57 new sp.]. Film, University of IIl.

1867. REPORT ON THE OSTRACODA DREDGED AMONGST THE HEBRIDES. Brit: Assoc. Adv. Sci., Report for 1866 (1867), p.208-211. [Pontocypris acupunctata, Bairdia complanata, Cythere (?) subflavescens, ‥ emaciata, ‥ complexa, C. humilis, Bythocythere? flexuosa, Cytherella scotica, $\underline{\text {. }}$ laevis, n.spp.] Film, University of Illinois.

- 1867-187I. In LES FONDS DE LA MER, vol. 1, 1867-1871. Ostracods were described or identified by Brady in the following chapters:

Part I:

XIII. La mer à Noumea, p.54-59,pl.7, f.4-6. XIV. Quelques points de la côte septentrionale de Java, p. 59-70, pls. 7,8.

XV. Abords de l'ile North-Watcher, p.70-72, pls. 8, 10 .

XVI. Sondes de I'Union, de Billiton à Poulo-Condore, p.73-82, pls. 8-10.

XVII. Les rivages de 1'ile Maurice, p.82-88, p1.10. XVIII. Les escales des Messageries impériales dans la Méditerranée, p.88-91 [Aglaia described]. XIX. Côte de Sicile, p.91-95, pls. 10,12. 
- 1867-1871 (Continued)

XX. Les iles de Santorin et de Syra, p.95-101, pl.12.

XXI. Les Dardanelles et Constantinople, p.101-103, pl. $12,13$.

XXII. Syrne, Rhodes et Mersina, p.103-108.

XXIII. La côte de Syrie (...) p.109-115, pl.13.

XXIV. Port-Said et Alexandrie, p.115-118, pls.13, 14.

XXVI. La Nouvelle-Providence, p.122-127, pl.14.

XXVIII. Rade de Saint-Vincent du Cap-Vert, (supplément), p.136-146, pls. 17,18 .

XXIX. Um mot sur le golfe de Gascogne, p.146-151, p1.14.

XXX. Colon-Aspinwall (supplément), p.152-155, pls.18,19.

XXXI. Les estomostracés de Hong Kong, p.155-159, pl.16.

XXXII. Nouveaux entomostracés de Port-au-Prince, p.160$161, \mathrm{pl} .18,19$.

XXXIII. Quelques entomostracés de Maurice, p.161-163.

Part II:

II. Vera Cruz et Carmen, p.181-192, pl.27.

III. Trois Ostracodes de Saint-Vincent de Cap-Vert, p. 192, 193, pl.19.

IV. Les Deux-Frtres et Poulo-Penang, p.193-195, pl.28.

v. Halt-Bay (detroit de Magellan), p.195-202, pl. 19, 27,28 .

XII. Baie Fortescue (Détroit de Magellan, p.232-237, pl.30. XIII. Rade de Santiago de Cuba, p.237-240, pl.30. 
- 1867-1871 (Continued)

XIV. Nouveaux entomostracés des iles Lucayes, p.240241, pl. 32 .

XV. Retour à Vera-Cruz et à Carmen, p.243-244, pl.32.

XVI. Crustacés et mollusque nouveau de la côte d'Afrique, p.244-247, pl.32.

[I have been unable to verify dates at which the various chapters were issued. The title page of the volume carries the date 1867-1871. From references in other publications it appears that part I was issued in 1868 and part 2 perhaps in 1871]. Film of Philadelphia Academy of Science copy

18682. A SYNOPSIS OF THE RECENT BRITISH OSTRACODA. Intellectual Observer, vol. 12, p.110-130, pls. 1,2. London [Cypridopsis, Macrocypris, Limnocythere, Cylindroleberis, n.gen.]. Film, University of Illinois.

- 1868b. A MONOGRAPH OF THE RECENT BRITISH OSTRACODA. Linnean Society, London, Trans., vol. 26, p.353-495, pls.23-41. Entomoconchidae, n. fam., Eucythere, nom.nov., for Cytheropsis Sars; 35 n.sp., assigned to 11 genera]. [This date for this Monograph is given in the Royal Society Catalogue as 1870]. Film, University of Illinois. 
- 1869a. CONTRIBUTIONS TO THE STUDY OF THE

ENTOMOSTRACA. NO. IV. OSTRACODA FROM THE RIVER

SCHELDT AND THE GRECIAN ARCHIPELAGO. Ann. Mag. Nat. Hist. ser. 4, vol. 3, p. 45-50, pls. 7, 8. [9 new species]. Film, University of Wisconsin.

1870. NOTES ON ENTOMOSTRACA TAKEN CHIEFLY IN THE NORTHUMBERLAND AND DURHAM DISTRICT (1869). Nat. Hist. Soc. Northumberland and Durham, Newcastle-uponTyne, Trans., vol. 3, p.361-373, pls. 12-14.

[Potamocypris, Xiphichilus, n. gen., 3 n.sp.]. Film, University of Illinois.

- 1875. in LES FONDS DE LA MER, vol. 2, 1875.

Ostracods were reported or described in the following chapters:

Part I:

I. Géneralitiés zur le golfe de Gascogne, p.9-13; [a list of species in which Hesvechilus contortus may be a missprint for Schlerochilus.

IV. Recerches bathometriques dans la fosse de Cap-Breton, p. 19-28, a few species mentioned.

IX. Sur les Cypridinae de Cap-Breton et sur ceux des mers d'Europe, p.53-61, pl.5 [many species reported and a few figured].

IX. Exploration de la fosse de Cap-3reton en 1872, p. $65-84$ 
1878. A MONOGRAPH OF THE OSTRACODA OF THE ANTWERP CRAG. Zool. Soc. London, Trans, vol. 10, p.379-409, p18. 62-69. i 15 new species in a fauna of 49 species.]. Film, University of Illinois. - 1880. REPORT ON THE OSTRACODA DREDGED BY H.M.S. CHALLENGER DURING THĖ YEARS 1873-1876. Report Sc1. Results Voyage H.M.S. Challenger... Zoology, vol. I, part 3, p.1-184, pls. 1-44. Phlyetenophora, Bythocyoris. Crossophorus, n.gen., and $143 \mathrm{n.sp.]} \mathrm{Film,} \mathrm{Lib.} \mathrm{Conp.}$ [also L.S.U.]

- 1881-1886. in LES FONDS DE LA MER, vol. 4, part III, 1881-1886 [1886?] Ostracods were described by Brady in the following Chapters:

IV. Les Cmistacés-Ostracodes des Expéditions du Travailleur et du Talisman du 1881 à 1883,p.164166 [a list with a number of nude names].

VII. Les ostracodes nouveaux des explorations du Travailleur et du Talisman, p.194-200, p1s.12,14, 15. Macrocyoris siliquosa, Bairdia folini, B. affinis, Cythere ventricristata, C. Ienisculpta, C. silcifer, ‥ monocantha, ‥ scaberrima, Bradycinetus biscayensis, B. ctenorynchus, Sarsiella globulus, Paracyoris paleata. Film Philadelphia Academy [part] U. S. Nat. Mus. [part] lacks plate 12. 
- 1886a. NOTES ON ENTONOSTRACA COLLECTED BY MR. A. HALY IN CEYLON. Linnean Soc. London, Journ., vol. 19, p.293-371, pls. 12-21. [Cypris malcolmsoni, ‥ montifera, … luxata, $\underline{\text { c. purpurascens, }} \underline{\text { c. halyi }}$, $\underline{\text { c. }}$ tenuicaudata, ‥ furfuracea, n.spp.; Cyprinotus, n.gen., C. cingalensis, Cypridopsis marmorata, Bairdia tenera, Aglaia (?) acuminata, Cythere truncatula, C. fabacea, C. ruperti, $\underline{\text { C. subcuneata, }}$. . coralloides, $\underline{\text {. bima- }}$ millata, ‥ laqueata, Cytheridea orientalis, ‥ pusilla, Loxoconcha sagittalis, L. papillosa, L. elongata, Xestoleberis sulcata, Bythocythere retusa, Paradoxostoma, eingalense, n.spp.] Film, University of Illinois. - 1886b. NOTES ON FRESHWATER ENTOMOSTRACA FROM SOUTH AUSTRALIA. Zoological Soc. London, Proceedings, p.82-73, pls.8-10. [4 n.sp. of Cypris; I of Chlamydotheca; 1 of Cypridopsis; 1 of Notodromas; and 1 of Candona. Film, University of Illinois.

- 1890. ON OSTRACODA COLLECTED BY H. B. BRADY, Esq., L.I.D., F.R.S., IN THE SOUTH SEA ISLANDS. Royal Soc. Edinburgh, Trans., vol. 35, p.489-525, pls.1-4. [Streptoleberis, Pleoschisma, n.gen., and $48 \mathrm{n}$.species]. Film, University of Chicago.

- 1897. A SUPPLEMENTARY REPORT ON THE CRUSTACEANS OF THE GROUP MYODOCOPA OBTAINED DURING THE 'CHALLENGER' EXPEDITION, WITH NOTES ON OTHER NEW OR IMPERFECTLY KNOWN 
SPECIES. Zool. Soc. London, Trans. vol. 14, pt. 3, p. 85-100, pls. 15-17. [Cyclasterope, n.gen., and 6 n.sp.J. Film, University of Illinois.

- 1898a. ON NEW SPECIES OF OSTRACODA FROM NEW ZEALAND. ZooI. Soc. London, Proc. for 1898, p.203204. [Eupathistonia, Trachyleberis, n.gen., not described.]. Film, L.S.U.

- 1898b. ON NEW OR IMPERFECTLY-KNOWN SPECIES OF OSTRACODA, CHIEFLY FROM NEW ZEALAND. Zool. Soc. London, Trans, vol. 14, pt.8, p.429-452,pls.43-47. [Eupathistoma, Trachyleberis, n.gen., and 18 new species of various genera.] Film, University of Illinois. - 1902. ON NEW OR IMPERFECTLY-KNOWN OSTRACODA, CHIEFLY FROM A COLLECTION IN THE ZOOLOGICAL MUSEUM, COPENHAGEN. Zool. Soc. London, Trans,, vol. 16, pt. 4, p.179-210, pls. 21-25. [Cypridinodes, Codonocera n. gen., and 17 n.sp.] Film, University of Illinois. - 1906. ON THE CRUSTACEAN FAUNA OF A SALT-WATER POND AT AMBLE. Nat. Hist. Soc. Northumberland, Durham, and Newcastle upon Tyne, Trans, ser. 2, vol. 1, p.330336, pls. 9,10. [Proteocypris, n.gen. P. salina, n.sp.]. Film, University of California. 
1907a. ON ENTOMOSTRACA COLLECTED IN NATAL BY MR. JAMES GIBSON. (PART II). Natal Government Museum, Ann., London, vol. 1 [1908 on title page, but recorded in Zool. Record for 1907], p.173-186, pls. 29-32. [4 new species]. Film, University of Illinois. - 1907b. CRUSTACEA. OSTRACODA. in National Antarctic Expedition 1901-1904, vol.3, p.1-9, pls.1-3. [8 n.sp., and Linocheles, n.gen.]. Film, University of Michigan.

- 1910. A REVIȘION OF THE BRITISH SPECIES OF OSTRACOD CRUSTACEA BELONGING TO THE SUBFAMILIES CANDONINAE AND HERPETOCYPRIDINAE (WITH A NOTE, ON A PARISITIC WORM BY MISS. M. V. LEBOUR, M.Sc.). Zool. Soc. London, Proc., Jan.-March, 1910,p.194-220, pls.19-30 [Candona,

1 n.sp., I n.name; Siphlocandona n.gen., S. normani, n.sp.]. Film, University of Illinois.

- 1913a. ON TWO BRITISH ENTOMOSTRACA BELONGING TO THE ORDERS COPEPODA AND OSTRACODA. ZOOLOgical SOC. London, Proc. for 1913, p.231-234,pls.38-40. [Genus Aruneila described]. Film, University of Illinois. - 1913b. ON FRESHWATER ENTOMOSTRACA FROM VARIOUS

PARTS OF SOUTH AFRICA. Natal [Government] Museum, Pietermaritzburg, Annals, vol.2,p.459-474,pls.33-38, [London] [2 n.sp., and Hyalocypris, n.gen.] 
Brady, G. S., Crosskey, H. W., and Robertson, D. 1874. A MONOGRAPH OF THE POST-TERTIARY ENTOMOSTRACA OF SCOTLAND INCLUDING SPECIES FROM ENGLAND AND IRELAND. Paleontographical Society, London, pp. i-v, 1-274, pls.1-16. [Krithe, Bosquetia, n.gen., 131 species, 20 new; Darwinellidae, n.fam.] Film, University of Illinois.

Brady, G. S., and Norman, A. M. 1889. A MONOGRAPH OF THE MARINE AND FRESH-WATER OSTRACODA OF THE NORTH ATLANTIC AND OF NORTHWESTERN EUROPE. SECTION I. PODOCOPA. Royal Dublin Soc., Sci. Trans., ser. 2, vol. 4, p. 63-270, pls. 8-23. [Cyclocypris, Erpetocypris,

Scottia, Ilyocypris, Anchistrocheles, n. gen.;

Machaerina, nom.nov. for Xiphichilus; 22 new species; genotypes designated for many genera, some erroneously; Darwinulidae, Paradoxostomatidae, n. fams.] Film, Chicago.

- 1896. A MONOGRAPH OF THE MARINE AND FRESH-WATER OSTRACODA OF THE NORTH ATLANTIC AND OF NORTHWESTERN EUROPE. SECTIONS 2-4. MYODOCOPA, CLADOCOPA, AND PLATYCOPA. Royal Dublin Soc., ser.2, vol.5, p. 621-746, pls. 50-68. [Tetragonodon, n.gen., I.

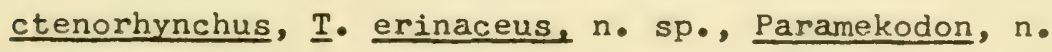
gen., P. inflatus, n.sp., Rutidermatidae, n. fam., Rutiderma, n.gen., R. compressa, n.sp•, Sarsiellidae, 
- 1896. (Continued).

n.fam., Nematohamma, n.gen., N. obliqua, n.sp.

Prionocypris, n.gen., Pionocypris, n.gen.]. Film, University of Illinois.

Brady, G. S. and Robertson, D. 1869. NOTES OF A WEEK'S DREDGING IN THE WEST OF IRELAND. Ann. Mag. Nat. Hist., ser.4, vol. 3, p. 353-374, pls. 18-22 [7 n.sp.] Film, L.S.U.

- 1870. THE OSTRACODA AND FORAMINIFERA OF TIDAL RIVERS. Ann. Mag. Nat. Hist., sér. 4, vol. 6, p. 1-33, pls. 41-10. [Goniocypris, Metacypris, Polycheles n. gen., and 15 n.sp. An important paper on ostracod ecology]. Film, University of Wisconsin.

1872. CONTRIBUTIONS TO THE STUDY OF THE ENTOMOSTRACA. NO. VI. ON THE DISTRIBUTION OF THE BRITISH OSTRACODA. Ann. Mag. Nat. Hist., ser. 4, vol. 9, p.48-70, pls. 1,2. [Darwinella, nom. nov. for Polycheles BER. 1870, preoccupied, and 3 n.sp. An important ecological paper.] Film, University of Illinois. - 1874. CONTRIBUTIONS TO THE STUDY OF THE ENTOMOSTRACA. NO. IX. ON OSTRACODA TAKEN AMONGST THE SCILLY ISLANDS, AND ON THE ANATOMY OF DARWINELLA STEVENSONI. Ann. Mag. Nat. Hist., ser. 4, vol. 13, p.114-118, pls. 4,5. Xestoleberis labiata, Cytherura 
fulva, Paradoxostoma cuneatum, n.sp.]. Film, University of Wisconsin.

Branson, C. C. 1935. FRESH-WATER INVERTEBRATES FROM THE MORRISON (JURASSIC?) OF WYOMING. Journ. Paleo., vol.9, no.6, p.514-522, pls.56, 57. [Morrisonia, n•gen•, M. wyomingensis, n.sp.]. Film, L.S.U.

- 1936. NEW NAME FOR A MORRISON OSTRACODE GENUS. Journ. Paleo., vol. 10, no.4, p.323. [Theriosynoecum for Morrisonia]. Film, L.S.U. - 1948. BIBLIOGRAPHIC INDEX OF PERMIAN INVERTEBRATES. Geol. Soc. America, Memoir 26, 1049 pp. [Conchostraca, p.846-854; Ostracoda, p.854-883.] L.S.U.

Brayer, Roger C. 1952. SALEM OSTRACODA OF MISSOURI. Jour. Paleo.,vol. 28, no. 2, p.162-174, pls.27-28, 4 text figs. [Brillius, Edita, new gen., $15 \mathrm{n.sp.,}$ and $3 \mathrm{n}$. vars.] Film, L.S.U.

Brehm, V. 1923. BERICHT ÜBER DIE VON DR. H. WEIGOLD IN CHINA GESAMMELTEN KOPEPODEN UND OSTRAKODEN. Internat. Rev. d. Gesamten Hydrobiol. u. Hydrographie, Leipzig, Bd. 11, p.329-345, 20 figs. [Drieschia, n. gen., 4 n.sp. and vars.] Film, University of Illinois. 
Brongniart, Charles. 1876. NOTE. SUR UN NOUVEAU GENRE D'ENTOMOSTRACÉ FOSSILE PROVENANT DE TERRAIN CARBONIFÈRE DES ENVIRONS DE SAINT-ÉTIENNE (PALEOCYPRIS EDWARDSII). Annales des Sciences Gélogiques, vol.7,no.3, 6pp., pl.6. Film made by L.S.U. from borrowed copy from Princeton.

Bronstein, F. 1930. BEITRÄGE ZUR KENNTNIS DER OSTRACODEN-FAUNA DES BAIKALSEES. Trav. Comm. étude Lac Bajkal, vol.3, p.117-157. Leningrad. Russian, with German summary. [Eucytheridea, n. subgen. of Cytheridea; Metacandona, n.gen.; $5 \mathrm{n} . \mathrm{sp}$. of Cytherissa, 2 of Metacandona, and 3 of Candona.] Film made by L.S.U. on copy from Yale.

Bronstein, Z. S. 1939. ON THE ORIGIN OF THE OSTRACODA FAUNA OF THE LAKE BAIKAL. Comptes Rendus (Doklady) Acad. Sci. de I.URSS., N.S., vol. 25, no. 4, p. 333337. [in English]. (Adademiia Nauk SSSR). Film, Harvard, 1948.

- 1947. FAUNE DE I'URSS. CRUSTACÉS. Vol. II, no.1. OSTRACODES DES EAUX DOUCES. Institut Zoologique de I'Académie des Sciences de l'URSS, N.S., No. 31. Moscou-Leningrad, 339pp., 206 text figs., 14 pages of plates on ostracoda. [Much reclassification of the Cypridae. The Cyprinae arranged in Tribes Notodromini, 
1947. (Continued).

Eucyprini, Cyprinotini, Herpetocyprini, Hungarocyprini,

Herpetocyprellini, Scottini, and Cypridopsini. The

Candoninae arranged in Tribes Cyclocyprini and Candonini. Twenty nine n.sp. and vars. An English summary accompanied by a key to families, subfamilies, tribes, and genera.] Film, L.S.U.

Canavari, M. 1899. OSTRACODI SILURIANI DI SARDEGNA. SOC. Toscana di Sci. Nat. di Pisa, Atti, vol. 11, (1897-98) 1899, p.150-153. [Entomis pteroides, n.sp., p.151] (The 1899 date from G. S. London, "Geological Literature added to the Geological Society Library for the year ended Dec. 31, 1899, and other sources. Date on title page of volume is 1897-98.) Film from University of Texas.

- 1900. FAUNA DEI CALCART NERASTRI CON CARDIOLA ED ORTHOCERAS DI XEA SANT'ANTONIO IN SARDINIA. Palaeontographica italica, vol.5,p.187-210, pls.25,26. [13 n.sp., or sp. fig'd for first time]. Film, L.S.U. Chapman, Frederick. 1901. ON SOME FOSSILS OF WENLOCK AGE FROM MULDE, NEAR KLINTEBERG, GOTLAND, WITH NOTES BY PROF. T. R. JONES AND DR. F. A. BATHER. Ann. Mag. Nat. Hist., ser.7, vol.7, p.141-160,pl.3, f.10-12. Film, L.S.U. 
- 1904. ON SOME FORAMINIFERA AND OSTRACODA FROM JURASSIC (LOWER OOLITE) STRATA NEAR GERALDTON, WESTERN AUSTRALIA. Royal Soc. Victoria, Proc. vol. 16 , (2 ser.) Pt. 2, p.185-206, pls. 22,23. [7 new sp. and vars.; Paradoxorhyncha, n. gen.] Film, Harvard.

Chapman, Frederick, and Sherborn, C. D. 1893. ON THE OSTRACODA OF THE GAULT AT FOLKESTONE. Geol. Mag•, dec. III, vol. 10, p. 345-349, pl. XIV. [8 n.sp. or vars.] Film, L.S.U.

Chyzer, Cornel. 1858. UEBER DIE CRUSTACEEN-FAUNA UNGARNS. Zool.-Botan. Gesell., Vienna, Verhandlungen, vol.8, p.505-518 [Cypris zenkeri, n. sp. Chyzer and Toth, with descriptions of 11 other species]. Film, University of California.

Claus, C. 1873. NEUE BEOBACHTUNGEN ÜBER CYPRIDINNEN. Zeitschr. f. Wissenschaftliche Zoologie, vol. 23, p.211-227, pls. 10-11. [Monopia n. gen., Cypridina monopia $=$ Monopia flaveola, n.sp.] Film, University of Chicago.

- 1874a. DIE FAMILIE DER HALOCYPRIDEN. Schriften Zoologischen Inhalts, Wien, Heft I, p.I-16, pls. I-16. [Halocypria, n. gen., H. globosa, n. sp.] Film, Crear Library• 
- 1874b. DIE GATTUNGEN UND ARTEN DER HALOCYPRIDEN. Zool.-Botan. Gesell., Vienna, Verhandlungen, vol. 24, p. 175-178. [3 n.sp. of Conchoecia; 1 of Halocypris; Halocypria, n•gen., H. globosa n.sp. Film, University of IIlinois.

- [1890] 1891. DIE GATTUNGEN UND ARTEN DER MEDITERRANEAN UND ATLANTISCHEN HALOCYPRIDEN NEBST BEMERKUNGEN ÜBER DIE ORGANISATION DERSELBEN. [1890] Arbeiten Zool. Inst. Wien und Zool. Stat. Triest, Vienna, vol. 9, 1891, p. 1-34 [ Conchoecinae, Halocyprinae, subfams. of Halocypridae; Paraconchoecia, Conchoecetta, Conchoecilla, Conchoecissa, Pseudoconchoecia, Mikroconchoecia, n.gen. and 13 n.sp.] Abst. Roy Micr. Soc. Jour. 1891, p. 344. Film, University of Illinois.

- 1891a. DIE HALOCYPRIDEN DES ATLANTISCHEN OCEANS UND MITTELMEERES. [K. Adad. Wiss., Wien] 83pp. 26 pls. [A very thorough anatomical study of the genera and species reported briefly by Claus, 1890. Extensive illustrations are provided for the previously unfigured species and genera.] Film, Crear Library.

- 1891b. DAS MEDIANAUGE DER CRUSTACEEN. Arbeit. Zool. Inst. Univ. Wien, u.d. Zool. Stat. Triest, vol. 9, p.225-266, pls. 14-17. [Eumonopia given as new name for Monopia Claus 1873, because of Lubbock's use of Monops; Candonella, n.gen. in footnote,p.231] Film, University of Illinois. 
- 1893. BEITRÄGE ZUR KENNTNISS DER SÜSSWASSER-OSTRACODEN, Arbeit, Zool. Inst, Univ. Wien, u. Zool. Sta. Triest, vol. 10, p. 147-216, pls. 1-12. The date is usually given as 1892, but the title page carried date of 1893. [Acanthocypris, A. bicuspis; Pachycypris, $\underline{\text { P. leuckarti, }}$. incisa, n. gen., n.sp., Heterocypris, n. gen.]. Film, University of Illinois.

Conrad, T. A. 1843. OBSERVATIONS ON THE LEAD BEARING LIMESTONE OF WISCONSIN, AND DESCRIPTIONS OF A NEW GENUS OF TRILOBITES AND FIFTEEN NEW SILURIAN FOSSILS. Acad. Nat. Sci. Philadelphia, Proc., vol. 1, p. 329-335. [Cytherina fabulites, n.sp., p. 332]. Film, I.S.U. Cooper, Chalmer I. 194I. CHESTER OSTRACODES OF ILLINOIS. Illinois St. Geol. Surv., Report of Investigation No. 77, 101pp. 14 pls. [Cribroconcha, Incisurella, Tetratylus, Paracavellina, Platychilus, Mesoglypha, Venula, Ectodemites, Polytylites, Proparaparchites, n. gen; Amphissitinae n. subfam., 359 species, many new.] Film, L.S.U.

1942. PLATYCHILELLA, NEW NAME FOR PLATYCHILUS COOPER. Journ. Paleo., vol. 16, no. 6, p. 777 . Film, L.S.U. - 1946. PENNSYLVANIAN OSTRACODES OF ILLINOIS. Illinois St. Geol. Survey, Bull. 70, 177 pp., 21 pls. 
- 1946. (Continued).

36 text figs. [Fabalicypris, Hastifaba, Paraparchitella, n.gen., and 106 new species.] Film, L.S.U.

- 1947. UPPER KINKAID (MISSISSIPPIAN) MICROFAUNA FROM JOHNSON COUNTY, ILLINOIS. Journ. Paleo., vol. 21, no.2, p.81-94, pls. 19-23. [3 n.sp.; Varix, n.gen.] Film, L.S.U.

Coryell, H. N. 1928a. SOME NEW PENNSYLVANIAN OSTRACODA. Journ. Paleo., vol. 2, no.2, p.87-94, pl.11.

[Seminolites, Cavellina, n.gen,, and 11 new species]. Film, I.S.U.

- 1928b. SOME NEW PENNSYLVANIAN OSTRACODA. Journ. Paleo., vol. 2, no.4,p.377-381,pl.51. [Hollinella, Glyptopleurina, n.gen., and 4 new species.] Film, L.S.U.

1930. JONESITES, A NEW NAME FOR THE OSTRACODE GENUS PLACENTULA. Journ. Paleo., vol. 4, no. 3, p. 294-295. Film, L.S.U.

Coryell, H. N. and Billings, Gladys D. 1932. PENNSYLVANIAN OSTRACODA FROM THE WAYLAND SHALE OF TEXAS. Amer. Midland Naturalist, vol.13, no.4, p.170-188, pls. 17, 18. [18 n.sp., Waylandella, Moorites, n.gen.] Film, L.S.U. 
Coryell, H. N., and Booth, R. T. 1933. PENNSYLVANIAN OSTRACODA: A CONTINUATION OF THE STUDY OF THE OSTRACODA FAUNA FROM THE WAYLAND SHALE, GRAHAM, TEXAS. Amer. Midland Nat., vol. 14, no.2, p.258-278, pls.3-5. [Girtyites, Kirkbyella, Silenites, Waylandella, Burlella, Artifactella, Birdsallella as new gen., and 16 new species] [Note: the name Kegelites is substituted for Girtyites by CEB. on the 2nd page of the table of contents of vol. 14] Film, University of Illinois.

Coryell, H. N., and Cuskley, Virginia A. 1934. SOME NEW OSTRACODES EROM THE "WHITE MOUND" SECTION OF THE HARAGAN SHALE, MURRAY COUNTY, OKLAHOMA. Amer. Mus. Novitates, no. 748,12 pp., 2 pls. [Bicornella, Parahealdia n.gen., and 17 new species]. Film, L.S.U.

Coryell, H. N., and Fields, Suzanne. 1937. A GATUN OSTRACODE FAUNA FROM CATIVA, PANAMA. Amer. Museum Novitates No. 959, 18pp., 2 pls. [Platella, Navecythere, Favella, Cativella, Caudites, Kangarina, Iuvula, Macrocytherina, Pellucistoma as new genera; Basslerites Howe, nom॰nov., and 15 new species]. Film, I.S.U.

Coryell, H. N., and Johnson, Samuel C. 1939. OSTRACODA OF THE CLORE, LIMESTONE, UPPER MISSISSIPPIAN, OF ILLINOIS. Jour. Paleo., vol. 13, no.2, p.214-224, pls. 25-26. [KIoedenellina, Lokius, Gillina, Glyptopleurites, Reversabella, Ceratopleurina, Sargentina, n.gen., and 24 n.sp.]. Film, L.S.U. 
Coryell, H. N., and Malkin, Doris S. 1936. SOME HAMILTON OSTRACODES FROM ARKONA, ONTARIO. Am. Mus. Novitates No. $891,20 \mathrm{pp} .2 \mathrm{pls}$. [Richina, Strepulites, Rudderina, Bufina, Bairdites, Ponderodictya, Spinovina, Quasillites. Janetina, Jenningsina described as new genera and Ropolonellidae, Quasillitidae as new families. Film, L.S.U.

Coryell, H. N., and Rogatz, Henry. 1932. A STUDY OF THE OSTRACODE FAUNA OF THE ARROYO FORMATION, CLEARFORK GROUP OF THE PERMIAN IN TOM GREEN COUNTY, TEXAS. Amer. Midland Nat., vol. 13, no.6, p.378-395, 2 pls. Notre Dame, Indiana. [Knoxina, Antiparaparchites, Ellipsella, n.gen., and 10 n.sp.] Film, L.S.U. Coryell, H. N., and Rozanski, George. 1942. MICROFAUNA OF THE GLEN DEAN LIMESTONE. Journ. Paleo., vol. 16, no.2, p.137-151, pls.23,24. [34 sp. and vars. of Ostracods, of which 31 are described as new. Pargraphylus, Hardinia, Healdioides, new genera]. Film, L.S.U.

Coryell, H. N., and Sample, C. H. 1932. PENNSYLVANIAN OSTRACODA. A STUDY OF THE OSTRACODA FAUNA OF THE EAST MOUNTAIN SHALE, MINERAL WELLS FORMATION, MINERAL WELLS, TEXAS. Amer. Midland Naturalist, vol.13, no.5, p. 245-281, pls.24-26. [Hollites, Sulcella, n.gen. and 23 species assigned to various genera]. Film, L.S.U. 
Coryell, H. N., Sample, C. H., and Jennings, P. H. 1935. BAIRDOPPILATA, A NEW GENUS OF OSTRACODA, WITH TWO NEW SPECIES. Amer. Mus. Novitates, No. 777, 5pp., 4 figs. Film, L.S.U.

Coryell, H. N., and Sohn, I.G. 1938. OSTRACODA FROM THE MAUCH CHUNK (MISSISSIPPIAN) OF WEST VIRGINIA. Journ. Paleo., vol. 12, no.6, p.596-603, pl.69. [Thirteen species, 11 being new; Microcoelonella, Persansabella, Geffenina, Geffenites, n. gen.J. Film, L.S.U.

Coryell, H. N., and Williamson, Marjorie. 1936. A STUDY OF THE OSTRACODA FAUNA OF THE WALDRON SHALE, FLAT ROCK CREEK, ST. PAUL, INDIANA. Am. Mus. Nat. Hist., New York, Am. Mus. Novitates No. 870, 7 pp., 15 figs. [Lindsayella, Cornulina, Aechminaria, Cyrtocypris, n.gen., spelled Cyrtocyprus once in error, and 15 n.sp.] Film, L.S.U.

Cossmann, Maurice. 1899. RECTIFICATIONS DE NOMENCLAUTRE (CORRECTIONS FAITES SUR LA TABLE DE LA SECONDE ANNÉE DE LA "REVUE CRITIQUE") Revue Crit. de Paléozoologie [et de Paléophytologie] Paris, vol.3, p.45. [Kirkbyia n.name for Synaphe, preoccupied]. Film, University of Michigan. 
Costa, 0. G. 1849. NESIDEA, NUOVO GENERE DI ENTOMOSTRACEI DELL'ORDINE DEGLI OSTRACODI O CIPROIDI. Accademia pontaniana, Naples, Atti, vol.5,p.183-188,pl.4. [Nesidea n. gen., N. hirta n.sp.] Film, L.S.U. of Princeton copy.

Croneis, Carey. 1939. TAXONOMY OF CHESTER OSTRACODES. Denison Univ., Granville, Ohio, Sci. Lab. Journal, Bull. vol.34,p.28-31. [April, 1939] [Idiomorphina, Croneis and Gale, n.name for Idiomorpha, CEG., and 4 new names for preoccupied species.] Film, I.S.U.

Croneis, Carey, and Bristol, Hubert M. 1939. NEW OSTRACODES FROM THE MENARD FORMATION. Denison Univ., Granville, Ohio, Sci. Lab. Jour., Bull., vol. 34, p.65-102, pls. 3,4. [Thirty-one new species, and Denisonia, n.gen.] Film, L.S.U.

- 1942. DENISONELLA, NEW NAME FOR DENISONIA. Journ. Paleo., vol. 16, no.6, p.777. Film, I.S.U.

Croneis, Carey, and Funkhouser, Harold J. 1939. NEW OSTRACODES FROM THE CLORE, FORMATION. DEnisOn Univ., Granville, Ohio, Sci. Lab. Jour., Bull., vol. 33, p. 331-350, pls. 9,10. [Lamarella, Carboprimitia, Neoklnedenella n.gen., and 25 new species.] Film, L.S.U. 
Croneis, Carey, and Gale, Arthur S., Jr. 1939. NEW

OSTRACODES FROM THE GOLCONDA FORMATION. DEnison Univ •, Granville, Ohio, Sci. Lab. Journal, Bull. vol. 33, p.251-295, pls. 5-6. Microparaparchites, Perprimitia, Pterocodella, Golcondella, Leightonella, Verrucosella, Discoidella, Workmanella, Glyptopleuroides, Idiomorpha, Bairdiolites, new gen.] Film, L.S.U.

- 1939b. IDIOMORPHINA, NEW NAME FOR IDIOMORPHA, CRONEIS AND GALE. Journ. Paleo., vol. 13, no.4, p. 466 [published in July]. Film, L.S.U.

Croneis, Carey, and Gutke, Ralph L. 1939. NEW OSTRACODES FROM THE RENAULT FORMATION. Denison Univ., Granville, Ohio, Sci. Lab. Journal, Bull. vol. 34, p.33-63,pls. 1,2. [Chesterclla, Hastacypris, and $30 \mathrm{n.sp.]}$. Film, L.S.U.

Croneis, Carey, and Thurman, Franklin A. 1938. NEW OSTRACODES FROM THE KINKAID FORMATION. Denison Univ., Granville, Ohio, Sci. Lab. Jour., Bull. vol.33, p.297330, pls.7,8. [Deloia, n.gen., and 27 new species.] Film, I.S.U.

Cushman, Joseph A. 1906. MARINE OSTRACODA OF VINEYARD SOUND AND ADJACENT WATERS. Boston Soc. Nat. Hist., Proc., vol.32, no.10, p.359-385,pls.27-38. [Pseudocytheretta, n.gen. and 10 n.sp.] Film, L.S.U. 
Daday, E. 1895 [Deesi Dr. Daday Jenö; or Dr. Eugen von Daday de Dees] A CYPROIS DISPAR (CHYZ) ANATOMIAI VISZONYAI. Termész. Füzetek, Budapest, vol. 18, columns $1-138,4$ pls. [each page with two columns: German and Hungarian]. Film from Library of Congress.

- 1898. MIKROSKOPISCHE SÜSSWASSERTHIERE AUS

CEYLON. Természetrajzi Füzetek, Budapest, Supplement to vol. 21, 123pp., figs. [Ostracoda p.69-85, figs. 34-40. [Stenocypris ceylonica, Cypris granulata, Cypricercus reticulatus, Notodromas entzi, n.spp.] Film, Library of Congress.

- 1900. A MAGYARORSZÁGI KAGYIÓSRÁKOK MAGÁNRAJZA. OSTRACODA HUNGARIAE, Budapest.p.1-320, many text

figures. [A monograph of Hungarian fresh-water ostracoda. Bibliography of 213 titles, P.21-33; Anatomy; Classification; Bairdiidae ancestral to Darwinulidae, Cytheridae, Cypridae, p.115; evolutionary diagram of Cypridae, p.118; Cyprinae, Candoninae, n.subfams•; Ctenocyprina, Cypridiformia n. tribes Zygopsida, Synopsida, Euopsida, Typhlopsida, n. subtribes; Iliocyprella, Eucandona, n. gen.] In Hungarian, with a latin key to all units discussed. Film made at L.S.U. of Philadelphia Academy copy • 
- 1904. MIKROSKOPISCHE SÜSSWASSERTHIERE AUS

TURKESTAN. Zool. Jahrblicher, Abt. Syst. Geog. u. Biol., vol. 19, p.469-553, pls.27-30 [Ostracods, p.514-530, pls. 29-30; 3 n.sp.] Film, Yale.

- 1905. UNTERSUCHUNGEN ÜBER DIE SÜSSWASSER-MIKROFAUNA PARAGUAYS. IV. OSTRACODA. ZOOIOgIca, vol.44,p.234-270, pls.15-19. [Tribes Ctenocyprina and Cypridiformia of subfamily Cyprinae, $9 \mathrm{n} . \mathrm{sp}$, and Cytheridella, n.gen.] Film, Library of Congress.

- 1908. ADATOK NÉMET-KELET-AFRIKA ÉDESVÍZI MIKROFAUNÁJANAK ISOMERE'TÉHEZ. Mathematikai és termeszettudomanyi ertesitö, [Magyar tud. akad.] Budapest, vol.26, p.1-57-200-220, 294-321, 374-42I, 455-474. [Mesocypris p.374-77; Pseudocypris, p.317-2];

Oncocypria, p.386-92 n.gen., plus many new species, later described and figured in Zoologica, 1910] Film from Brown University.

- 1909. BEITRÄGE ZUR KENNTNIS DER FAUNA TURKESTANS AUF GRUND DES VON D.D. PEDASCHENKO GESAMMELTEN MATERIALS. V. OSTRACODEN UND PLANKTON DER SEEN ISSYK-KUL UND TSCHATYR-KUL. Leningradskoe obshchestvo estestvoispytatelei, Trudy [St. Petersburg, Trav. Soc. Nat. Sect. Zool. 39, 2(1-e partie) p.1-58, I pl.] [Herpetocyprella, n.gen. H. mongolica, n.sp., Cypricercus mongolicus.]. Film, L.S.U. from Philadelphia Academy. 
19102. DIE SÜSSWASSER-MIKROFAUNA DEUTSCH-OST-AFRIKAS. Zoologica, Stuttgart, Heft 59 (Bd. 23, Ifg. 1-5) p.1-314, 18 Taf. [Ostracod descriptions p.139-235, pls.9-16. Pseudocypris, p.193, Mesocypris, p.197, Oncocypria, $0.214 \mathrm{n}$. gen . and many new species belonging to numerous genera. Same paper as Daday 1908, but this time in German and with figures.] Film, Library of Congress.

1910b. ERGEBNISSE DER MIT SUBVENTION AUS DER ERBSCHAFT TRAITL UNTERNOMMENEN ZOOLOGISCHEN FORSCHUNGSREISE DR. FRANZ WERNER'S NACH DEM ÄGYPTISCHEN SUDAN UND NORD-UNGANDA. XV • BEITRÄGE ZUR KENNTNIS DER MIKROFAUNA DES NIIS. Akad, der Wissenschaften, Vienna, Math. Nat. KI. Sitzungsberichte, vol. 119, p. 537-568, pls. 1-3. [6 new species; Aglaie1]a, n.gen.] Film, L.S.U. Dana, J. D. Nov. 8, 1849. CONSPECTUS CRUSTACEORUM QUAE IN ORBIS TERRARUM CIRCUMNAVIGATIONE, CAROLO WILKES E. CLASSE REPUBLICAE FEDERATAE DUCE., I.EXIT ET DESCRIPSIT JACOBUS D. DANA. PARS II. Amer. Acad. Arts and Sci. vol. 2 [May 1848-May 1852] p. 9-51. [Ostracods, p.4953. Conchaccia, n.gen. and $14 \mathrm{n.sp.]}$ Abst. in Amer. Jour. Sci., ser. 2, vol. 8, 1849, p.276-285. 
- 1853. CRUSTACEA. United States Exploring Expedition. During the years 1838, 1839, 1840, 1841 , 1842, under the command of Charles wilkes, U.S.N., vol. XIV, Part II, Philadelphia, p.1277-1618, with Atlas of 96 plates. [Tribe III. Cyproidea=0stracoda, p.1277-1304, pls. 90-91. The first attempt at a classification into families and subfamilies. Halocypridae, n.fam.; Cyprinae, Cytherinae, Cypridininae, Halocyprinae, n.subfamilies, and Halocypris, n.gon.; all assigned to the Tribe Cyproidca.l Film, Library of Congress.

Delachaux, Theodore. 1328. FAINNE INVERTÉBRÉE D'EAU DOUCE DES HAUTS PLATEAUX DU PÈROU. (RÉGION DE HUANCAVELICA, DÉPARTMENT DE JUNIN) RÉCOLTÉE EN 1915 PAR FEU ERNEST GODET, ING. (CALANIDES, OSTRACODES, ROTATEURS NOUVEAUX). Soc. Neuchateloise des Sci. Nat., N.S., vol. 1, p.45-77, 73 figs. on 11 pls. [7 n.sp., Neolimnocythere, Paracythereis, n.gen.] Film, University of Illinois.

DeIo, David M. 1930. SOME UPPER CARBONIFEROUS OSTRACODS FROM THE SHALE BASIN OF WESTERN TEXAS. Journ. Paleo,, vol. 4, no.2, p.152-178, pls. 12,13. [22 new species; Acratia, Kellettella, n.gen. Film, L.S.U.

Dobbin, Catherine N. 1941. FRESH-WATER OSTRACODA FROM WASHINGTON AND OTHER WESTERN LOCALITIES. UnIV • Of Washingtion, Publ. in Biology, vol.4, no.3, p. 175-246, p1s.1-14. [Cyclocypria, n.gen. and 15 n.sp. and vars.] Film, L.S.U. 
Dons, J. A., and Henningsmsen, G. 1949. TWO NEW MIDDLE ORDOVICIAN OSTRACODS FROM OSLO. Norsk Geologisie Tidsskrift, vol. 28, p.27-32. [U1lia Henningsmoen, n.gen., U. ulli, U. holtedahli Dons, n.spp.l Film, L.S.U.

Dubowsky, N.F. 1939. ZUR KENNTNISS DER OSTRACODENFAUNA DES SCHWARZEN MEER. Trav. Sta. biol., Karadagh, vol. 5,p.3-68, 68 figs. [Russian with German summary. Pseudocytherura, Pontocythere, n.gen., and 9 n.sp., vars., or names.] Film, University of California.

Edwards, Richard A. 1944. OSTRACODA FROM THE DUPLIN MARL (UPPER MIOCENE) OF NORTH CAROLINA. JOUR. Paleo., VOl. 18, no.6, p.505-528, pls.85-88. [Campylocythere, Acuticythereis n.gen.; 39 sp. and vars., 32 of which are new]. Film, L.S.U.

Egerov, V. G. 1950.

E Г платфориы. I. Hoedenellidae. Гостоптехиздат, 1950.

[Not seen: but from Polenova 1952, it is evident that it contains at least: Cavellininae, Dizygopleurinae, Knoxinae as new subfamilies, and Donellina, Evlanella, Knoxiella , Knoxites, Kalugia, as new genera]. [OSTRACODA OF THE FRANSKOGO STRATA OF THE RUSSIAN PLATFORM. I. KLOEDENELI.IDAE]. 
Ekman, Sven. 1914. BEITRÄGE ZUR KENNTNIS DER SCHWEDISCHEN SÜSSWASSER-OSTRACODEN . Upsala. Universitet. Zool. Bidrag från Uppsala, Bd. 3, p.l-36, figs. [Nannocandona, n.gen., N. faba, n.sp.]. Film from Notre Dame.

Eichwald, Ed. 1857. BEITRAG ZUR GEOGRAPHISCHEN VERBREITUNG DER FOSSILEN THIERE RUSSLANDS. Soc. Imp. Nat. Moscou [I. Mosk. Obs. Ispytatelei, Prirody] Bull., vol. 30, pt. 2, no.4, p.307-353. [Ostracoda p.306-313; eleven n.sp. Cyclas as subpenus of Cytherina]. Film, University of Minnesota.

Elofson, Olof. 1939. NEUE UND WENIG BEKANNTE CYTHERIDEN VON DER SCHWEDISCHEN WLSTKÜSTE. Arkiv för Zoologi, Stockholm, Bd. 30A, no.21, p.1-22, 33 figs. Cytherominae n.subfam.; Bythocythere intermedia, n.sp. Film, University of Illinois.

- 1941. SUR KENNTNIS DER MARINEN OSTRACODEN SCHWEDENS MIT BESONDERER BERÜCKSICHTIGUNG DES SKAGERAKS. Upsala Univ., Zool. Bidrag från Uppsala, vol. 19, p.215-534, 52 text figs., 42 maps. [Heterocyprideis, Hirschmannina, n.gen.; Paracythereis, Heterocythereis, Hemicytherura, n.subgen.; Xestoleberis pusilla, n.sp. An excellent ecologic study]. Film, University of Illinois. 
Emmons, Ebenezer. 1855. AMERICAN GEOLOGY, CONTAINING A STATEMENT OF THE PRINCIPLES OF THE SCIENCE WITH FULI ILLUSTRATIONS OF THE CHARACTERISTIC AMERICAN FOSSILS. Vol. I, Part 2, published at Albany, N.Y. [Crustacea, p.208-221 [Beyrichia regularis, B. ciliata, Cytherina subelliptica, $\underline{\text { c. crenulata, }}$ c. subcylindrica, n.spp.] Film, L.S.U. of Smithsonian copy.

Fischer, Sebastian. 1854. ABHANDLUNG ÜBER DAS GENUS CYPRIS, UND DESSEN IN DER UMGEBUNG VON ST. PETERSBURG UND VON FAL.L BEI REVAL VORKOMMENDEN ARTEN. ACad. Imp. Sci. St. Petersburg [Akademiia Nauk, SSSR] Mém. des Sav. Etrangers, vol. 7, p.127-167, pls.1-1l. Film, University of Illinois.

- 1855. BEITRAG ZUR KENNTNISS DER OSTRACODEN. [Bayer] Akad. Wiss., Munich, Math.-Phys. Cl.VII, Bd. 3, p.635-666, pls.1,2. [Paradoxostoma n.gen. and 16 n.sp.] Film, University of Illinois.

Fitton, William Henry. 1836. OBSERVATIONS ON SOME OF THE STRATA BETWEEN THE CHALK AND THE OXFORD OOLITE, IN THE SOUTH-EAST OF ENGLAND. Geol. Soc. London, Trans (2) vol.4,p.103-388,pls.11-23. [Cypris granulosa, C. tuberculata listed, and $\underline{C}$. valdensis $1=C$. faba Ann. of Phil. 1824, pl.8, f.376 ..) named and discussed; and described by Sowerby in Appendix A., p.344, pl.21.] Film, L.S.U. 
Foerste, August F. 1918. THE RICHMOND FAUNAS OF LITTLE BAY DE NOQUETTE IN NORTHERN MICHIGAN. Ottawa Naturalist, vol. 31 (1917) p.97-103; (1918) p.121-127. [Bollia permarginata n.sp., p.124] Film, University of Illinois.

Forbes, S. A. 1893. A PRELIMINARY REPORT ON THE AQUATIC INVERTEBRATE FAUNA OF THE YELLOWSTONE NATIONAL PARK, WYOMING, AND OF THE FLATHEAD REGION OF MONTANA, United States Fish Comm. Bull. for 1891, p.207-258, pls.37-42. Published April 29, 1893. [Ostracoda, p.244-246, pls. 37-38; Cypris barbatus, n.sp.1. Film, L.S.U.

Forel, F. A. 1894. ZOOLOGIE LACUSTRE. Bibliotheque Universelle. Archives des Sciences Physiques et Naturelles, vol.32,p.588-605. [Limnocytheridea, p.602] Film, Yale.

Fuchs, Alexander. 1915. DER HUNSRÜCKSCHIEFER UND DIE UNTERKOBLENZSCHICHTEN AM MITTRLRHEIN (LORELEIGEGEND). Prussia. Geol. Landes., Abh., n.s., vol. 79, p.1-80, pls.1-18. Berlin. [Beyrichia tetrapleura, pl.77, pl.18,f.11-13]. Film, University of California. - 1920. BEITRAG ZUR KENNTNIS DER DEVONFAUNA DER VERSE- UND DER HOBRÄCKER SCHICHTEN DES SAUERLANDISCHEN FACIESGEBIETES. Prussia. Geol. Landes., Jahrb. fur das Jahs 1918 [1920], pt.1, p.58-95, pls.5-9 
1920. (Continued).

[Ostracods, p.81-83,pl.6. Gibba n.subpen. of Beyrichia,

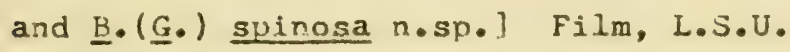

Furtos, N.C. 1933. THE OSTRACODA OF OHIO. Ohio State Univ., Ohio. Biol. Survey, Bull. vol. 5, no.6, (Bulletin 29), $.412-524$, pls.1-16. [Candncypria n.gen., 32 n.sp. and vars., new tribes and groups.] Film, Harvard.

- 1936a. ON the ostracoda fRom the CENOTES of YUCATAN AND VICINITY. Carnegie Inst. Washington Publ. no.457, p.89-115, 88 figs. [13 new species]. Film, University of Illinois.

- 1936b. FRESH-WATLR OSTRACODA FROM FLORIDA AND NORTH CAROLINA. Amer. Midland Nat., vol. 17, no. 2, p.491-522, 15 figs. [9 new species]. Film, L.S.U.

Geis, H. L. 1932. SOME OSTRACODES FROM THE SALEM LIMESTONL, MISSISSIPPIAN, OF INDIANA. Journ. Paleo., vol.6, nn.?, p.249-188, pls. 22-26. i46 species, 43 new; Oliganisius, Savagella, Microcheilus, n.gen.] Film, L.S.U.

- 1933. MICROCHEILINEILA, A NEW NAME FOR THE OSTRACODE GENUS MICROCHEILUS. Journ. Paleo., vol.7, no.1, p.112. Film, L.S.U. 
Girty, George H. 1910. NEW GENERA AND SPECIES OF CARBONIFEROUS POSSILS FROM THE FAYETTEVILLE SHALE OF ARKANSAS. New York Acad. Sci., Annals, vol. 20, p.189-238. Paraparchites nichlesi cyclopea, Primitia fayettevillensis, P. seminalis, Halliella? reteriformis, Kirkbya lindahli arkansana, K. oblonga transversa, $\underline{K}$. reflexa, K. simplex, Amphissites rugosus, n.gen.n.sp•, Glyptopleura, n.gen., G. inopinata, G. angulata, Bairdia attenuata, B. cestriensis granulosa, n.spp. Glyptuplnuridae, Kirkbyidae, n.fams.J Film, Columbia University.

Goerlich, Franz. 1953. OSTRAKODEN DER CYTHERIDEINAE AUS DER TERTI ̈̈REN MOLASSE BAYERNS. Senckenbergiana vol. 34, p.117-148, 9 pls. Lyprideis? glabra, C. ?rara, Cytheridea (Cyth.) mulleri truncatula, … (…) bavarica, C.. (‥) eggeri, $\underline{C}$. (…) leingartensis, $\underline{C}$. (. .) ventricosa, ‥ (Eucytheridea) reticulata, Haplocytheridea dacica elegantior, Paracyprideis triebeli, P. punctata, n.sp. and vars.] Film, I.S.U.

Goldenberg, Fr. 1870. ZWEI NEUE OSTRACODEN UND EINE BLATTINA AUS DER STEINKOHLENFORMATION VON SAARBRÜCKEN. Neues Jahrb. Min. Geol. Pal., 1870, p.286-289, woodcut, figs 1-4. [Cyprida elongata n.gen., n.sp.] Film, L.S.U. 
Granata, Leopoldo, and Caporiacco, Lodovico di. 1949. OSTRACODES MARINS RECUILLIS PENDANT LES CROISIÈRES

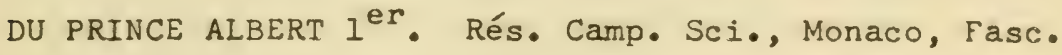
109, p.1-51, pls.1-4. [A comprehensive revision of the family Halocypridae; Metaconchoecia, Orthosonchoecia, Macroconchoecia, new gen., and 49 species, 2 new.] Film, I.S.U.

Grekoff, N. 1951. QUELQUES OSTRACODES NOUVEAUX DU SÉNONIEN SUPÉRIEUR DU CAMEROUN. Inst. Français du Pétrole, Revue, vol. $6, p .53-59, p l s .1,2$. [3 new species, Ovacytheridea, n.gen.] Film, L.S.U.

Groom, Theodore. 1902. ON POLYPHYMA, A NEW GENUS BELONGING TO THE LEPERDITIADAE FROM THE CAMBRIAN SHALES OF MALVERI. Geol. Soc. London, Quart. Journ., vol. 58, p.83-88, p1.3. Film, L.S.U.

Gümbel, [C.] W. 1874. BRIEFWECHSEI [Ostracoden in Stringocephalenkalk von Paffrath; iber Dactyloporideen und Gyroporellan .... Roy. Soc. Catalogue, X, p.87] Neues Jahrb. Min. Geol. Pal., p.68-70. [numerous ostracods from Paffrath discussed]. Film, I.S.U.

Gürich, George. 1896. DAS PÄLAOZOICUM IN POLNISCHEN MITTELGEBIRGE. Russ. Kais. Min. Gesell., St. Petersburg, Verh., ser.2, vol.32,p.1-539,pls.1-15,map. [Ostracods, p.374-391,p1.10,15; Antitomis, Polyzygia, Poloniella, 
Gürich, George. 1896. (Continued).

Richterina, n•gen.; $20 \mathrm{n} . \mathrm{sp}$, or new names]. Film, University of Minnesota.

Giithorl, Paul. 1934. DIE ARTHROPODEN AUS DEM CARBON UND PERM DES SAAR-NAHEPFALZ-GEBIETES. Preuss. Geol. Landes. Abh., n.f., Heft 164, 219 pp., 30 pls. [Ostracods, p.9-12, pl.1; Candona elongata (Goldenberg, 1870) and Cuselina impressa v. Ammon 1310]. Film, L.S.U.

Hadding, Assar. 1913. UNDRE DICELIOGRAPTUSSKIFFERN I SKÂNE JAMTE NÅGRA DÄRMED EKVIVALENTA BILDNINGAR. Lund Universitet, Lund, Sweden. Arsskr., n.s., pt.2, 9, no.15; Kongl. Fysiofr. Sällsk. Handl., N.S. 2l, no.115; Lunds Geol. Fältklubb, ser. b, no.6, [Ostracoda, p.67,

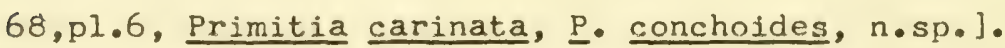
Film, Crear Library.

Hall, James. 1852. DESCRIPTIONS OF THE ORGANIC REMAINS OF THE LOWER MIDDLE DIVISION OF THE NEW YORK SYSTEM. NEW York (State) Natural History Survey, Paleontology of New York, vol.2, 362 pp., pls. [Ostracoda, p.14, 301 , 317, 338, pls.4,66,67,78] Film, L.S.U.

- 1860. DESCRIPTIONS OF NEW SPECIES OF FOSSILS, FROM THE HAMILTON GROUP OF WESTIRN NEW YORK, WITH NOTICES OF OTHERS FROM THE SAME HORIZON IN IOWA AND INDIANA. New York State Cabinet of Natural History. 
- 1860. (Continued).

Ann. Rept. no. 13, p.76-94, 15 text figs. [Leperditia punctulifera, n.sp. p.92]. Film, Yale.

Hall, James, and Whitfield, R. P. 1875. DESCRIPTION OF INVERTEBRATE FOSSIIS, MAINLY FROM THE SILURIAN SYSTEM. Ohio Geol. Survey, Rept.2,p.2, Paleontology, p.65-157 [Ostracods, p.101-]05, p1.4. 6 species; Beyrichia quadrilirata new.] Film, L.S.U.

Hamilton, Irving B. 1942. OSTRACODES FROM THE UPPER PERMIAN OF Texas. Jour. Paleo., vol. 16, no.6, p.712-718, p1.110, 1 text fig. [13 n.sp., and Alveus, n.gen.] Film, L.S.U.

Harding, J.P., and Sylvester-Bradley, P.C. THE OSTRACOD GENUS TRACHYLEBERIS. British Museum (Natural History) Bulletin, Zoology, vol.2, no.1, $15 \mathrm{pp} ., 2 \mathrm{pls}$. [ Trachyleberis scabrocuneata redescribed, with lectotype chosen; T. Iytteltonensis, n.sp.]. Film, I.S.U. of my copy.

Harlton, Bruce H. 1929a. PENNSYLVANIAN OSTRACODA FROM MENARN COUNTRY, TEXAS. Texas Univ. Bull. 29C1, p.139161, pls. 1-4. Austin, Texas. [22 n.sp.; Bairdianella, n.gen.] Film, I.S.U. 
- 1929b. SOME UPPER MISSISSIPPIAN (FAYETTEVILIE)

AND LOWER PENNSYLVANIAN (WAPANUCKA-MORROW) OSTRACODA OF OKLAHOMA AND ARKANSAS. Amer. Journ. Sci., ser. 5, vol. I8, no. 105, p.254-270, pls.1,2. [Genus Bassleria and 19 new species]. Film, L.S.U.

\section{MICROPALEONTOLOGY OF THE PENNSYLVANIAN}

JOHNS VALLEY SHALE OF THE OUACHITA MOUNTAINS, OKLAHOMA, AND ITS RELATIONSHIP TO THE MISSISSIPPIAN CANEY SHALE. Jour. Paleo., vol.7, no.l, p.3-29, pls.1-7. [Healdiidae, n.fam., Acohmine 1la, Mooreina, n.gen., and 12 n.sp.] Film, L.S.U.

Harper, Frances, and Sutton, A. H. 1935. OSTRACODES OF THE MORRISON FORMATION FROM THE BLACK HILLS OF SOUTH DAKOTA. Jour. Paleo., vol. 9, no.8, p.623-628, pl.76. [I.n.sp., I n.var.]. Film, L.S.U.

Harris, Reginald W. 1931. DESCRIPTION OF OSTRACODES AND CONODCNTS in: The Stratigraphy and physical characteristics of the Simpson group. Oklahoma Geol. Surv. Bul1. 55, p.87-95, pls.3,5,11,14. [Bromidella, n.gen., and $10 \mathrm{n.sp.J.} \mathrm{Film,} \mathrm{L.S.U.}$

Harris, R. W., and Lalicker, Cecil G. 1932. NEW UPPER CARBONIFLROUS OSTRACODA FROM OKLAHOMA AND KANSAS. Amer. Midl. Naturalist, vol.13, no.6, p.396-409, pls. 36,37. Notre Dame, Indiana. Albanella, Coryella, Sansabelloides, n.gen., and 13 n.sp. and vars.J Film, L.S.U. 
Hartwig, W. 1899a. CANDONA ELFLECTELLA (ROBERTSON 1880) BILDET EINE SELBSTÄNDIGE GATTUNG. Zool. Anzeiger, vol. 23, p.309-311. [Paracandona, n.gen.] Film, L.S.U.

- 1899b. 1. ÜBER EINE NEUE CANDONA AUS DER PROVINZ

BRANDENBERG: CANDOAT MARCHICA, UND 2. ÜBER DIE WAHRE CANDONA PUBESCENS (KOCH). Gesell. Naturf. Freunde, Berlin. Sitzungsber. No.8, p.183-189, text figs. Film, University of California.

Henningsmoen, Gunnar. 1948. THE. TRETASPIS SFRIES OF THE KULlatoRP CORE. Upsala Univ., Min.-Geol. Inst. Bull., vol. 32, p.374-432, pls.13-15. ilo n.sp. and Kinnekullea, n. gen]. Film, L.S.U.

- 1950. UILERELIA, NEW NAME: REPLACING ULLTA HENNINGSMOEN, 1949. Norsk Geologisk Tidsskrift, vol. 28, p.244. Film, L.S.U.

- 1953. CLASSTFTCATION OF PALEOZOIC STRAIGHT-HINGED OSTRACCDS. Norsk Geol. Tidsskpift, vol.31, p.185-288, pls. 1-2. [Paleocona, new suborder; Eurychilinidae, Sigmoopsiidae, new as families; Beyri-hiopsinae, Glyptopleurinae, Glossopsi inae, Leperditellinae, Quasillit,inae, Tetradellinae, Ropolonellinac, Conchoprimitiinae, Eridocnnchinae, Sigmoonsiinae, new as subfams.; Hesslandella, Kinnckullea, Sigmoopsis, 


\section{- 1953 (Continued).}

Sigmobolbina, Carinobolbina, Bolbina, Piretopsis,

Platybolbina, n•gen.; Piretellidae of Opik changed to Pirctellinae, subfam. of Tetradellidae]. Primitiopsinae, subfam. of Euxychilinidae. Film, L.S.U.

- 1953b. THE MIDDLE ORDOVICIAN OF THE OSLO REGION, NORWAY. 4. OSTRACODA. Norsk Geologisk Tidsskrift, Bd. 32, p.35-56, pls. 1-5. [ 18 species, 9 new, Piretopsis, n.gen.] Film, L.S.U.

- 1954a. LOWER ORDOVICIAN OSTRACODS FROM THE OSLO REGION, NORWAY. Norsk Geol. Tidsskrift, vol. 33, p.4168, pls. 1,2. [Nanopsis, n.gen.; Conchorprimitia eos, C. socialis vulgaris n.sp., n. var., J Film, L.S.U. - 1954b. UPPER ORDOVICIAN OSTRACODS FROM THE OSLO REGION, NORWAY. Norsk Geol. Tidsskrift, vol. 33, p. 69-108, pls. 1-6. Bol.bina tuberculata, Opikium porkuniensis, Primitia nslcensis, Platybolbina tiara, Laccoshilina tarda, Bollia ascentuata, Pseudulrichia norvegica, Monoseratella bos, n.spp., Important discussion of genotype of Platybolbina.] Film, L.S.U.

Henry, Marguerite. 1923. A MONOGRAPH OF THE FRESH-WATER ENTOMOSTRACA OF NE.W SOUTH WALES. PART III. OSTRACODA. Linnean Soc. New South Wales, Sydney, Proseedings, vol. XLVIII, part 3, no. 192, p.267-286, pls.24-29. i a key 
Henry, Marguerite. 1923 (Continued)

to the classification of genera and species, with 4 new species]. Film, Library of Congress.

Hesse, M. 1878. DESCRIPTION DES CRUSTACÉS RARE OU NOUVEAUX DES COTES DE FRANCE. [DESCRIPTION DE NOUVEAUX CRUSTACÉS DE LA LÉGION DES BRANCHIOPODES, DE I'ORDRE DES CLADOCÈRES, FORMANT LA NOUVELIE FAMILLE DES COPÉCHÉTIENS (1) ET LE NOUVLAU GENRE COPECHAETE, NOBIS.] Ann. Sci. Nat. Zool. 6th. ser., vol. 7, article 14 ( 15 in table of contents), pl.12. (Family "Copéchétiens" and genus Copechaete, new). Film, I.S.U.

Hessland, Ivar. 1949. INVESTIGATIONS OF THE LOWER ORDOVICIAN OF THE SILJAN DISTRICT, SWEDEN. I. LOWER ORDOVICIAN OSTRACODS OF THE SILJAN DTSTPICT, SWEDEN • Upsala Universitet. Min-geol. inst. Bull. vol. 33, p.97-408, pls.1-18. [62 n.sp.; Conchoides, Pinnatulites, Conchoprimites, Euprimites, Laccochilina, Aulacopsis, Glossopsis, Ogmoopsis, n.gen.; Euprimitiinae, n.subfam. of Hollinidae. Primitiopsinae, a subfam. of Primitiidae.] Film, L.S.U. - 1954. GLOSSOMORPHITES, A NEW GENERIC NAME FOR GLOSSOPSIS HESSLAND, 1949, PREOCCUPIED. (OSTRACODA). Norsk Gen]. Tidsskrift, vol. 32, h.2-4, p.227. [A]so Glossomorphitinae, n.name for Glossopsinae Henningsmoen 1953). Film, L.S.U. 
Hirschmann, Nikolaj. 1909. BEITRAG ZUR KENNTNIS DER OSTRACODENFAUNA DES FINNISCHEN MEERBUSENS. SOcietas pro Fauna et Flora Fennica, Meddelanden, vol.35, p.282297, pl.figs.1-12. [Cyclocypris impressopunctata, Cytheridea fennica, Cythere ilyophila, Cytheromorpha albula, Cytheromorpha claviformis, n.sp., and Cytheromorpha, n•gen.] Film, Univ. of Illinois.

- 1912. BETTRAG ZUR KENNTNIS DER OSTRAKODENFAUNA DES FINNISCHEN MEERBUSENS. SWEITE MITTEILUNG. Societas pro Fauna et Flora Fennica, Helsingfors, Acta, 36, no.2, p.1-65, pls.1-3, 15 figs. [Candona Ievanderi, Loxoconcha sarsi, n.sp., nom.nov.]. Milm, Univ. of Illinois.

N.M.KNIPOVITCH AND S.A. PAVLOVITCH IN THE SUMMER OF 1908 Lin Russian; the translation of the title as given from Blake 1933, p.241\} Akademiia Nauk, SSSR. Leningrad, ZoologicheskiY Musel, Ezhegodnik [Annuaire Musée Zool. Imp. Acad. Sei.] Vol. 20, p.569-597, 27 figs. Palmenella, n.gen., p.580. Film, Library of Congress.

Hof. , G. Clayton. 1942a. THE OSTRACODS OF ILLINOIS. THEIR BIOL.CGY AND TAXONOMY. Illinois Univ., Urbana. Illinois Biol. Monographs, vol. 19, p.5-196, pls.1-9. [A good discussion of the ecology of fresh-water ostracods, and of their morphology. The taxonomic portion is accompanied by 
Hoff, C. Clayton. 1942a (Continued).

a key. Eleven new species, and several new combinations. Notodrominae a change in spelling of Notodromadinae Kaufmann.] Film, University of Illinois.

- 1942b. THE SUBFAMILY ENTOCYTHERINAE, A NEW SUBFAMILY OF FRESH-WATER CYTHERID OSTRACODA, WITH DESCRIPTIONS OF TWO NEW SPECIES OF THE GENUS ENTOCYTHERE. Amer. Midland Nat., vol.27, p.63-73, 13 figs. Film, I.S.U. Holland, Wilbur C. 1934. THE OSTRACODS OF THE NINEVEH IIMESTONE OF PENNSYLVANIA AND WEST VIRGINIA. Pittsburgh, Carnegie Institute, Museum, Annals, vol.22, p.343-350, p1.25. [Whipplella, n.gen., with $7 \mathrm{n.sp.]} \mathrm{Film,}$ University of Michigan.

Hornibrook, N. de B. 1949. A NEW FAMILY OF I.IVING OSTRACODA WITH STRIKING RESEMBLANCF. TO SOME PALEOZOIC BEYRICHIIDAE. Royal Soc. New Zealand, Trans. vol. 77, pt.4, p.469471, pls.50, 51. Puncijdae, n.fam., Puncia novozealandica n. gen., n.sp•; Manawa trjphena, n•gen., n.sp.] Film, L.S.U.

1952. TERTIARY AND RECENT MARINE OSTRACODA OF NEW ZEALAND. New Zealand Geol. Surv., Paleo. Bull. 18, $82 \mathrm{pp} ., 18 \mathrm{pls}$. [New genera and subgenera: Loxocythere, Arcacythere, Bradleya, Quadracythere, Aversovalya, Miracythere, Bythoceratina, Cytheralison, Saida, $\$ 80$ new 
species and subspecies; Trachyleberinae, subfam. of. Cytheridae]. Film, L.S.U.

Howe, Henry V. 1947. REVISION OF THE OSTRACOD GENUS

BUNTONIA. Amer. Assoc. Petrol. Geol., program of 1947 meeting, Los Angeles, California, p.50 [abst.] Microfilm, L.S.U.

- 1951a. NEW NAME FOR GENOTYPE OF ALATACYTHERE (OSTRACODA) Jour. Paleo., vol. 25, no. 4, p.538. Film, I.S.U.

- 1951b. NEW TERTIARY OSTRACODE FAUNA FROM LEVY COUNTRY, FLORIDA. Fla. St. Board of Conservation, Geol. Surv. Bull. no. 34, pl.1-43, 5 pls. [30 new species; Aulocytheridea, Nephokirkos, Urocythere, Spongicythere, Occultocythereis, Hirsutocythere, Leniorythere, n.gen.] Film, L.S.U.

Howe, Henry V., and Chambers, Jack. 1935. LOUISIANA JACKSON EOCENE DITRACODA. Dept. of Conservation, Louisiana Geol. Surv., Geol. Bull. no.5, 65pp.,6 pls. [Buntonia, n.gen., and 38 n.sp.l Film, I.S.U.

Howe, Henry V., and Law, John. 1936. LOUISIANA VIC.KSBURG OLIGOCENE OSTRACODA. Dept. Conserv., Louisiana Geol. Survey, Geol. Bull. no.7, 96pp., 6 pls. [7] sp. and vars of which 66 are new; Archicythereis, Leguminocythereis, Pyricythereis, n.gen.] Film, I.S.U. 
Howe, H. V., and graduate studontst. 1935. OSTRACCDA OF

THE ARCA ZONE OF THE CHOCTAWHATCHEE MIOCENE OF FLORIDA. Florida Board Conservation, Geol. Bull. no.13, p.1-37, pls.1-4.

[tChambers, Jack; Brown, Bates; McGuirt, James; Neill, Wenzel; Hough, Leo; Ellis, Albert D.,Jr•; Stephenson, Morton; Spurgeon, Julia; Pyent, Lloyd; Dohm, Christian; Hadley, Wade; Taylor, Ralph; arri Johnson, T. J.] [2I n.sp., and vars,; Basslerella, n.gen.] Film, L.S.U.

Howe, Henry V., and Law, Jar. 1936. LOUISIANA VICKSBURG OLIGOCENE OSTRACODA. DOpi. Of Conservation, Louisiana Geol. Surv., Geol. Bull. No. 7, 96pp., 6 pls.

[Archicythereis, Leguninocythereis, Pyricythereis, n.gen., and 67 n.sp. and vars.] Film, L.S.U.

Israelsky, M. C. 1929 and 1935. UPPER CRETACEOUS OSTRACODA OF ARKANSAS. Arkansas Geol. Survey. Published in 1929 as an extract from Bul1. No.2, p.1-28, pls.1A-4A. Bulletin No. 2, Oil and Gas Geology of the Gulf Coastal Plain in Arkansas, by W. C. Spooner was published privately by Parke-Harper Printing Co., Little Rock, Arkansas, and Israelsky's paper with some revisions, was reprinted in 1935 as an Appendix, p.475-496, pls. IA-IVA. [24 new species]. Film. L. S. U. 
Jennings, P. H. 1936. A MICROFAUNA FROM THE MONMOUTH AND BASAL RANCOCAS GROUPS OF NEW JERSEY. BUIl. AmEr. Paleont., Ithaca, vol. 23, no.78, p.161-234, pls.1-7. [ 18 n.sp., Antibythocypris, Paracythereis, Pseudocythereis, n.gen.] Film, L.S.U.

Johnson, W. R. 1936. THE OSTRACODA OF THE MISSOURI SERIES IN NEBRASKA. Nebraska Geol. Surv. Paper No. 11, 52 pp., 5 pls. [Geisina, Kirkbyites, n.gen., K. upsoni, n.sp.] Film, L.S.U.

Jones, T. Rupert. 1849. A MONOGRAPH OF THE ENTOMOSTRACA OF THE CRETACEOUS FORMATION OF ENGLAND. Palaeontographical Soc. London, 1849, 40pp., 7 pls. [The genus Cythere divided into subgenera Cythereis, nov., Bairdia, M'Coy, and Cytherella, nov. 26 species, and several vars.; 13 being new]. [note: Sylvester-Bradley $1948 \mathrm{~d}$ gives the date as 1850; but the paper carries 1849 on title page.] Film, I.S.U.

- 1850a. DESCRIPTION OF the ENTOMOSTRACA OF the LATE PLEISTOCENE BEDS OF NEWBURY, COPFORD, CLACTON, AND GRAYS. Ann. Mag. Nat. Hist., ser. 2, vol.6, p.25-28, pl.3. [ 2 n.sp. Cypris, 1 of Candona, and 1 of Cythere.] Film, University of Illinois. 
- 1850b. CLASS CRUSTACEA, In William King's "A

Monograph of the Permian Fossils of England",

Palaeontographical Soc., London, p.58-66, pls.6,17,18.

[6 n.sp. described as ostracods, and $2 \mathrm{sp}$. described

as Phyllopods which later were referred to the ostracod Kirkbya]. Film, L.S.U.

- 1855a. NOTES ON PALAEOZOIC BIVALVED ENTOMOSTRACA. NO. I, SOME SPECIES OF BEYRICHIA FROM THE UPPER SILURIAN LIMESTONES OF SCANDINAVIA. Ann. Mag. Nat. Hist., ser. 2, vol. 16, p.81-92,pl.5. [divided into 3 groups: Simplices, Corrugatae, and Jugosae; $10 \mathrm{n.sp}$. and vars.]

1855b. NOTES ON PALAEOZOIC BIVALVED ENTOMOSTRACA. NO. 11. SOME BRITISH AND FOREIGN SPECIES OF BEYRICHIA. Ann. Mag. Nat. Hist., ser. 2, vol. 16, p.163-176, pl.6. [II n.sp. and vars.] Film, L.S.U.

. 1856a. NOTES ON THE ENTOMOSTRACA [ENTOMOSTRACA OF THE OSBORNE AND HEMPSTEAD SERIES[. In Edward Forbes' "ON THE TERTIARY FLUVIO-MARINE FORMATION OF THE ISLE OF WIGHT. Great Britain Geol. Survey, Memoir, p.157-158, plate 7. [Cytherideis, n. gen. C. unisulcata, $\underline{\text {. }}$ unicornis, and Candona forbesii n.sp.] Film from Yale. 
- 1856b. NOTES ON THE PALAEOZOIC BIVALVED

ENTOMOSTRACA. NO. III. SOME SPECIES OF LEPERDITIA. Ann. Mar. Nat. Hist., ser. 2, vol. 17, p. 81-10l, pls. 6,7. ileperditidae, n.fam. of Phyllopoda?, and $3 \mathrm{n} . \mathrm{sp}$. of Leperditia.] Film, L.S.U.

- 1857. A MONOGRAPH OF THE TERTIARY ENTOMOSTRACA OF CNGLAND. Palaeontographical Soc. London (1856) 1857 , p.1-58, pls.1-6. [ $1 \mathrm{n} . \mathrm{sp}$. of Candona, 14 of Cythere, 2 Cythereis, 5 of Cytheridea, 5 of Cytherideis, 1 of Bairdia, 1 of Cytherella. Cyprideis n. subgen.? of Cythere? Cotherideis n. subgen. of Cythere.] Film, L.S.U. - 1858a. NOTES ON THE PALAEOZOIC BIVAI.VED ENTOMOSTRACA. NO. IV. SOME NORTH AMERICAN SPECIES. Ann. Mag. Nat. Hist., ser.3, vol.1, p.24l-257, pls.9,10. [ 8 now sp. and vars. of Beyrichia; 7 of Leperditia; Isochilina, n. subgen. of Leperditia, and $3 \mathrm{n} . \mathrm{sp}$. of Cytheropsis.] Film, L.S.U.

- 1858b. ADDITIONAL NOTES ON PALAEOZOIC BIVALVED ENTOMOSTRACA FROM CANADA. Ann. Mag. Nat. Hist., ser.3, $\mathrm{v} \cap 1.1, \mathrm{p} \cdot 340-342 . \quad$ Leperditia canadensis anticostiana, L. amygdalina described as new, and corrections to Jones 1858a.] Film, L.S.U.

- 1858c. ON THE PALAEOZOTC BIVALVE ENTOMOSTRACA OF CANADA. Canada Geological Survey: Figures and Descriptions of Organic Remains, Decade III, p.91, 102 , 
- 1858c. (Continued).

pl.11. [Leperditia canadensis, vars. labrosa, loucklana, pauquettiana, josephina, anticostiana; Isochilina, n. subgen. of Leperditia.j Film, L.S.U.

- 1861. [DESCRIPTION OF ENTOMIS, n.gen, and E. TUBEROSA n.sp.] in THE GEOLOGY OF THE. NEIGHBORHOOD OF EDINBURGH, by H. H. Howell and Archibald Geike, Geol. Survey Great Britain, Memoir 32-Scotland, Appendix, p. 137, p1.2. [Entomis divisa MSS. is mentioned as smaller, but not described]. Film, University of California.

- 1864. DESCRIPTION OF ENTOMOSTRACA FROM THE

MOUNTAIN LIMESTONE OF BERWICKSHIRE AND NORTHUMBERLAND, WITH NOTES ON THE STRATA IN WHICH THEY OCCUR BY GEORGE TATE. Berwickshire Nat. Club. Proc. [Hist] 1863-1868 [1868 on title page of vol.], p.83-89, 3 figs. (Reprinted in "Geology and Archaeology of the Borders," by George Tate, 1865, p.15-21 acc. Bassler \& Kellett 1934, p.114. [Beyrichia tatei, n.sp. p.87]. Film, L.S.U. from Ia. St. Copy •

- 1865. MICROZOA OF THE VALLEY DEPOSITS OF THE NAR, NORFOLK. Geol. Mag., London, vol.2, p.306-307. [4 new species of Ostracods listed as determined by Brady, one of which was termed Normania carinata]. [ilm, L.S.U. 
- 1870a. ON ANCIENT WATER-FLEAS OF THE OSTRACODOUS

AND PHYLLOPODOUS TRIBES (BIVALVED ENTOMOSTRACA). ROyaI Microsc. Soc., Monthly Micros. Journal, London, vol.4, p.184-193. [Table of Classification, p.186-187 with Tribe II Cyproidea (Ostracoda) classed in 8 families: Cypridae; Cytheridae; Cypridinadae; Entomoconchidae;

Conchnecidae; Polycopidae; Cytherellidae; Leperditiadae. An extended discussion of the genera referred to the Leperditiadae.] Film, I.S.U.

1870d. ON SOME BIVAIVED ENTOMOSTRACA FROM THE COAL-MEASURES OF SOUTH WALES. Geol. Mag., London, vol. 7, p.214-220, pl.9. [Carbonia n.gen. and several n.sp. and vars.] Film, L.S.U.

- 1873a. ON ANCIENT WATER-FLEAS OF THE DSTRACODOUS AND PHYLLOPODOUS TRIBES (BIVALVED ENTOMOSTRACA). PART II. CYPRIDINADAE. PART III. POLYCOPIDAE, CYTHERELLIDAE, M. BARRANDE'S NFW GENERA, AND ENTOMIDAE. PART IV. CYPRIDAE AND CYTHERIDAE. PART V. PHYLLOPODA. MonthIy Micr. Jour., London, vol.10, p.71-78. [Rhombina, Cypridinella, Cypridellina, Offa n. gen., and Entomidae, n. fam.] Film, L.S.U.

- 1873b. ON SOME BIVALVE ENTOMOSTRACA, CHIEFIY CYPRIDIDAE, OF THE CARBONIFEROUS FORMATIONS. (This paper has been withdrawn by permission of the Council.) [Abstract.] Geol. Soc. Lstion, Quart. Journ. vol.29, p.409- 
1873b. (Continued).

412. Cypridinella n.gen. described p.410 with 7 species, one of which C. monitor carries a brief description and is indicated as typical. Cypridellina n.gen. with 8 species and 2 vars, described, but all through obvious error with the name Cypridinellad (p.410). Offa n.gen. with one species, ‥ barrandiana, p.409. Sulcuna n.gen. with 2 species, p.411. Rhombina, n.gen. with 2 sp. p.411.] Film, L.S.U.

- 1873c. NOTES ON THE PALAEOZOIC BIVALVED ENTOMOSTRACA. No. X. ENTCMIS AND ENTCMIDELIA. Ann. Mag. Nat. Hist., ser.4, vol. 1], p.413-4I7 [15 species of Entomis discussed; Entomidella, n.gen., based on 2 species.] Film, I..S.U.

- 1874. ÜBER ENTOMIS UND EIN NEUES GENUS RICHTERIA. Neues Jahrb. f. Min. Geol. u. Pal., 1874, p.180. [This is in the form of a letter, but title is given in table of contents of volume. Richteria, n.gen.] Film, L.S.U.

1879. NOTES ON THE PALAEOZOIC BIVALVED ENTOMOSTRACA. NO. XIII. ENTOMIS SERRATOSTRIATA AND OTHERS OF THE SO-CALLED "CYPRTDIEEN" OF THE DEVONIAN SCHISTS OF GERMANY • AnR. Mag. Nat. Hist., ser.5, vol.4, p.182-187, pl.11. [Richteria Jones $1874=$ Entomis $]$ Film, L.S.U. 
1881a. LIST OF SPECIES (PROVISIONAL) OF BIVALVE ENTOMOSTRACA, EC. COLLECTED BY MR. J. SMITH OF KILWINNING, FROM THE UPPER SILURIAN FORMATIONS OF SHROPSHIRE [p.7375] In Notes On a collection of Bivalved Entomostraca and other Microzoa from the Upper Silurian strata of the Shropshire District by J. Smith. Geol. Mag., n.s., dec.2, vol.8, p.70-75. Film, I.S.U.

- 1881b. ENTOMOSTRACA IN COAL-SHALES. Geol. Mag. dec. 2, vol.8, p.95. [Carbonia fabulina, C? bairdioides etc.) Film, L.S.U.

- 1881C. NOTES ON SOME PALAEOZOIC BIVALVED ENTOMOSTRACA. Geol. Mag., n.s. dec.2, vol.8, p.337-347, pls.9,10. [Cyprosis haswellii n.gen. and sp.; Cyprosina whidhornei n. gen., and sp., Beyrichia holli n.sp., and B. colwallensis Holl, MS., n.sp.J Film, L.S.U.

- 1881d. NOTES ON THE PALAEOZOIC BIVALED

ENTOMOSTRACA NO. XII. SOME CAMBRIAN AND SILURIAN IEPERDITIAE AND PRIMITIAE. Ann. Mag. Nat. Hist., ser.5, vol.8, p.332-350, pls.19,20. [3 n.sp. and vars. of Leperditia]. Film, L.S.U.

- 1883. PALAEOZOIC PHYLLOPODA; AS REPORTED TO THE. BRITISH ASSOCIATION, SOUTHPORT, 1883, SECTION C. GEOLOGY. Geol. Mag., dec.2, vol.10, p.461-464. [Places Barrande's genera Crescentilla, Aristozoe, Orozoe, Callizoe with the Phyllopoda]. Film, L.S.U. 
1884a. NOTES ON THE PALAEOZOIC BIVALVED

ENTOMOSTRACA. NO. XVIII. SOME SPECIE.S GF ENTONIDAE. Ann. Mag. Nat. Hist., ser.5, vol.14, p.391-4.03, pl.15. [3 n.sp. of Entomis, 1 of Bolbozoe, and Entomidel]a buprestis (Salter) designated as "a good type of that genus"]. Film, I.S.U.

- 1885a. NOTES ON THE LATE MR. GEORGE TATE'S SPECIMENS OF LOWER CARBONIFEROUS ENTOMOSTRACA FROM BERWICKSHIRE AND NORTHUMBERLAND. BErwickshire Nat. Club. [History] Proc., 1882-1884 [1885] p.313-325, pl.2. Alnwick. [Bernix, n.gen.] Film, Ia. St. College. - 1885b. ON SOME FOSSIL ENTOMOSTRACA FROM THE PURBECK FORMATION AT BOUIOGNE. Geologists Assoc., Proc., vol.8, p.54-59. [principally Cythere boloniensis Jones] Film, L.S.U.

- 1885\%. ON THE OSTRACODA OF THE PURBECK FORMATION. Ann. Mag. Nat. Hist., ser.6, vol.16, p.231-232, ahst. [gives many of the names of spectes described in Jones 1885e]. Film, L.S.U.

- 1885d. ON THE OSTRACODA OF THE PURBECK PORMATION: WITH NOTES ON THE WEALDEN SPECIES. Geol. Mag. DeC. 3, vol. 2, p.326, abst. [similar to $1885 \mathrm{c}$ in using names of species described in 1885 e, but with different spelling.] Film, L.S.U. 
- 1885e. ON THE OSTRACODA OF THE PURBECK FORMATION: WITH NOTES ON THE WEALDEN SPECIES. Geol. Soc. London, Quart. Jour., vol. 41, p.311-353, pls.8.9. [Cypridea punctata posticalis, $\underline{\text { C. dunkeri, }}$. ventrosa,

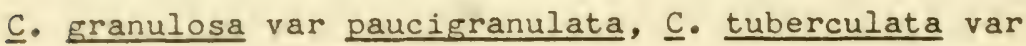
adjuncta, n.sp. and vars.; Cyprione, n.gen., C. bristovil, n.sp.; Metacypris forbesii and var. verrucosa n.sp. and var.; Darwinula Brady and Robertson, n. name for Darwine 11a, p.346; Cythere boloniensis changed to Candona bononiensis, C. ansata n.sp.; Cythere retirugata n.sp.] Film, L.S.U.

- 1887. NOTES ON SOME SILURIAN OSTRACODA FROM GOTHLAND. 8 vo. Stockholm. [12 n.sp. and vars., Primitiopsis, Bursulella, n.gen.] Film, Library of Congress.

- 1887b. NOTES ON THE PALAEOZOIC BIVALVED

ENTOMOSTRACA. NO. XXIV. ON SOME SILURIAN GENERA AND SPECIES (continued). Ann. Mag. Nat. Hist., ser. 5, vol. 19, p.400-416, pls.12, 13. [Thlipsura, 4 n.sp. or vars; Octonaria, n.gen., with 9 n.sp. and vars.; 2 of Bollia, I of Primitia, 3 of Aechmina.] Film, L.S.U. 18882. NOTES ON THE PALAEOZOIC BIVALVED ENTOMOSTRACA. NO. XXV. ON SOME SILURIAN OSTRACODA FROM GOTHLAND. Ann. Mag. Nat. Hist., ser.6, vol.1, p.395- 
- 1888a. (Continued).

411, pls.2],22. [4 n.sp. and vars. and many discussed and figured; Bursulella redescribed, and genotype $\underline{E}$. triangularis figured]. Film, L.S.U.

- 1888b. NOTES ON THE PALEOZOIC BIVALVED

ENTOMOSTRACA. - NO.XXVI. ON SOME NEW DEVOHIAH OSTRACODA. WITH A NOTE ON THEIR GEOLOGICAL POSITION, BY THE REV. G. F. WHIDBORNE, M.A., F.G.S. Ann. Mag. Nat. Hist,, ser. 6, vol.2, p.295-299, pl.11. [Kyammodes whidborne1 n.gen., n.sp. and 2 vars.] Film, I.S.U.

- 1889a. ON SOME PALAEOzOIC OSTRACODA FROM

PENNSYLVANIA, U.S. American Geologist, vol.4, p.337-342,

1 pl. [8 new sp.] Film, L.S.U.

- 1889b. NOTES ON THE PALAEOZOIC BIVALVED

ENTOMOSTRACA. NO. XXVII. ON SOME NORTH AMERICAN (CANADIAN) SPECIES. Ann. Mag. Nat. Hist., ser.6, no.3, p.373-387, pls.16-17. [Primitia scaphoides, n.sp., Beyrichia kloedeni acadica, n.var., Isochilina labrosa, n.sp., Aparchites whiteavesii, n.gen., n.sp., p.384. Film, L.S.U.

- 1889c. ON SOME PALAEOZOIC OSTRACODS FROM MORTH AMERICA, WALES, AND IRELAND. Geol.Mag., n.s., dec.3, vol.6, p.576 abst. [A number of new species listed as nude names]. Film, L.S.U. 
- 1890a. ON SOME PALAEOZOIC OSTRACODA FROM NORTH AMERICA, WALES AND IRELAND. Geol. Soc. London, Quart. Jour., vol.46, p.1-31, pls.1-4. [3 new Primitia, I new Strepula, 3 new Beyrichia, 2 new Isochilina, I new Leperditia, and I new Xestoleberis.J Film, I.S.U.

- 1890b. ON SOME DEVONIAN AND SILURIAN OSTRACODA FROM NORTH AMERICA, FRANCE, AND THE BOSPHORUS. GEOI. Soc. London, Quart. Journ., vol.46, p.534-556, pls.20, 21. [8 n.sp., Ulrichia, n.gen.] Film, L.S.U.

- 1891. CONTRIBUTIONS TO CANADIAN MICRO-PALAEONTOLOGY.

PART III. ON SOME OSTRACODA FROM THE CAMBRO-SILURIAN, SILURIAN, AND DEVONIAN ROCKS. Canada Geol. Survey [Geol. and Nat. Hist. Survey] Contrib to Canadian Micro-palaeontology, 1891, p.59-99, pls.10-13 [29 n.sp. and vars.; Leioditia Ulrich MS. $=$ Elpe, p.94] Film, L.S.U. from Boston Public Library.

- 1893a. ON SOME FOSSIL OSTRACODA FROM S.W.WYOMING, AND FROM UTAH, U.S.A. Geol.Mag., dec.3, vol.10, p.385-39l, pl.15. [13 n.sp.] Film, L.S.U.

- 1901. OSTRACODA [Table of Classification] in Frederick Chapman: ON SOME FOSSILS OF WENLOCK AGE FROM MULDE, NEAR KLINTEBERG, GOTLAND, WITH NOTES BY PROF. T. R. JONES AND DR. F. A. BATHER. Ann.Mag.Nat.Hist., ser.7, vol.7, p.14.1-160, pl.3, f.10-12. [Jones' portion p.146147; Aparchitinae, n.subfam. of Leperditiidae]. Film,L.S.U. 
Jones, T. Rupert, and Holl, H. B. 1865. NOTES ON THE

PALAEOZOIC BIVALVED ENTOMOSTRACA. NO. VI. SOME SILURIAN SPECIES (PRIMITIA). Ann. Mag. Nat. Hist., ser.3, vol.16, p.414-425, pl.13. [Primitia, n.gen., with 27 species, 18 new]. Film, L.S.U.

- 1869. NOTES ON PALAEOZOIC BIVALVEd ENTOMOSTRACA, No. 9. SOME SILURIAN SPECIES. Annals and Mag.Nat.Hist., ser.4, vol.3, p.211-229, pls.14, 15. [2 n.sp. of Cythere; Thlipsura, n.gen, and 3 n.sp.; Cytherellina, n. gen.; Aechmina, n.gen, and 2 n.sp.; 1 n.sp. of Beyrichia, and 2 of Primitia, Noorea, Jones and Kirkby, MS, n.gen., M. silurica, n.sp.] Film, L.S.U.

1886a. NOTES ON THE PALAEOZOIC BIVALVED

ENTOMOSTRACA. NO•XX. ON THE GENUS BEYRICHIA AND SOME NEW SPECIES. Ann. Mag. Nat. Hist., ser.5, vol.17, p.337-363, p1.12. [Bollia, Kloedenia, n.gen., and 7 n.sp. and vars.] Film, L.S.U.

- 1886b. NOTES ON THE PALAEOZOIC BIVALVEd ENTOMOSTRACA. NO. XXI. ON SOME SILURIAN GENERA AND SPECIES. Ann. Mag. Nat. Hist., ser.5, vol.17, p.407-414, pls.13, 14. [Strepula, Placentula, n.gen., and $14 \mathrm{n.sp}$. and vars.] Film, L.S.U. 
Jones, T. Rupert, and Kirkby, J. W. 1865. NOTES ON THE PALAEOZOIC ENTOMOSTRACA NO.V. MUNSTER'S SPECIES FROM THE CARBONIFEROUS LIMESTONE. Ann. Mag. Nat. Hist., ser.3, vol.15, p.404-410, pl.20. [3 n.sp. included]. Film, L.S.U.

- 1866. NOTES ON THE PALAEOZOIC BIVALVED ENTOMOSTRACA. NO. VII. SOME CARBONIFEROUS SPECIES. Ann. Mag. Nat. Hist., ser.3, vol.18, p.32-51. [Kirkbya annectens, n.sp., p.42; this paper is essentially a history of, and revision of previous ostracod studies on the Carboniferous]. Film, I.S.U.

- 1867. ON THE ENTOMOSTRACA OF THE CARBONIFEROUS ROCKS OF SCOTLAND. Geol.Soc.Glasgow, Trans. vol.2, p.213-228. [a list of 56 species, with localities. A number of specific names appear here for the first time.] Film, University of Minnesota.

- 1874. A MONOGRAPH OF THE BRITISH FOSSIL BIVALVED ENTOMOSTRACA FROM THE CARBONIFEROUS FORMATIONS by Professor T. Rupert Jones, F.R.S., G.S., Ec. Ec.; James W. Kirkby, ESQ.Ec.Ec.; and George S. Brady, ESQ., C.M.Z.S., Ec.Ec. Part I. THE CYPRIDINADAE AND THEIR ALLIES. By Prof. T. Rupert Jones, F.R.S., G.S., and J. W. Kirkby, Esq., Ec.Ec. Palaeontographical Society, London, p.1-56, pls.1-5. [8 n.sp. of Cypridina; Cypridinella n.gen, and 
- 1874. (Continued).

7 n.sp.; Cypridellina, n.gen., 8 n.sp. 2 n.vars., which had all been used under the name Cypridinella by Jones 1873b; Philomedes $1 \mathrm{n} \cdot \mathrm{sp} \cdot$; Rhombina n.gen. and 2 n.sp•; Entomoconchus 2 n.sp.; Offa n.gen., I n.sp.; Polycope, I n.sp. Note: These genera and most of the species had been used, and some partially described in one or the other of Jones 1873 papers!] Film, L.S.U.

1879. DESCRIPTIONS OF THE SPECIES OF THE OSTRACODUS GENUS BAIRDIA M.COY, FROM THE CARBONIFEROUS STRATA OF GREAT BRITAIN. Geol. Soc. London, Quart. Journ. vol.35, p.565-581, pls.28-32. Film, L.S.U.

- 1885. NOTES ON THE CARBONIFEROUS OSTRACODA OF THE NORTHWEST OF ENGLAND. Geol. Mag., n.s. Dec. 3, vol.2, p.535-541. [Phreatura concinna gen. and sp. nov., in checklist, not described, p.540]. Film, L.S.U.

1886a. ON SOME FRINGED AND OTHER OSTRACODA FROM THE CARBONIFEROUS SERIES. Geol. Mag., N.S. Dec.III, vol.3, p.433-439, pls.11,12. [Beyrichiopsis, Beyrichiella, n.gen., and 5 new species]. Film, L.S.U.

- 1886b. NOTES ON THE PALAEOzOIC BIVALVED

ENTOMOSTRACA. -NO. XXII. ON SOME UNDESCRIBED SPECIES OF BRITISH CARBONIFEROUS OSTRACODA. Ann.Mag.Nat.Hist., ser.5, vol.18, p.249-269, pls.6-9. [22 species]. Film, L.S.U. 
- 1886c. NOTES ON THE DISTRIBUTION OF THE

OSTRACODA OF THE CARBONIFEROUS FORMATIONS OF THE, BRITISH ISLES. Geol.Soc.London, Quart.Journ. vol.42, p.496514. [Phreatura, Youngia, n.gen.] Film, L.S.U.

- 1887. A IIST OF THE GENERA AND SPECIES OF BIVALVED ENTOMOSTRACA FOUND IN THE CARBONIFEROUS FORMATIONS OF GREAT BRITAIN AND IREIAND, WITH NOTES ON THE GENERA AND THEIR DISTRIBUTION. Geologists Assoc., Proc., vol.9, p.495-515. [Phreatura, Youngia, n.gen.] Film, L.S.U.

- 1895. NOTES ON THE PAIAEOZOIC BIVALVED ENTOMOSTRACA. NO. 32. SOME CARBONIFEROUS OSTRACODA FROM YORKSHIRE. Ann. Mag. Nat. Hist., ser.6, vol.16, p.452-460, p1.21. [Ycungiella, new name, for Youngia Jories and Kirkby 1886, not Lindstrom 1885]. Film, L.S.U. - 1898. ON CARBONIFEROUS OSTRACODA FROM IRELAND.

Royal Dublin Society, Sci. Trans. vol.6, ser.2, p.173200, pls.11, 12. [ 7 n.sp. and vars.; Synaphe, n.gen.] Film, University of Chicago.

Jones, T. R., Kirkby, J. W., and Brady, G. S. 1884. A MONOGRAPH OF THE BRITISH FOSSIL BIVALVED ENTOMOSTRACA FROM THE CARBONIFEROUS FORMATIONS. PART I. NO.2. THE CYPRIDINAE AND THEIR ALLIES. Palaeontographical SOC. 
Jones, T. R., Kirkby, J. W., and Brady, G. S. 1884. (Continued).

Monograph, p.57-92, pls.6,7. London. [14 new species and vars. of Cytherella, ]. of Philomedes, and 3 of Entomis] Film, L.S.U.

Jones, T. R., and Sherborm, C. D. 1888. ON SOME OSTRACODA FROM THE FULLERS-EARTH OOLITE AND BEDFORD CLAY • Bath Nat. Hist. and Antiquarian Field Club, Proc., Bath, vol.6, p.249-278, pls.1-5. [57 n.sp.] Film from Yale.

Juday, Chauncey 1907. OSTRACODA OF THE SAN DIEGO REGION. II. LITTORAL FORMS. University of California Publ. Zoology, vol.3, no.9, p.135-150, pls.18-20. [5 new species;

Paracytheroma, n.gen.] Film, University of Illinois.

Jurine, Louis. 1820. HISTOIRE DES MONOCLES, QUI SE TROUVENT AUX ENVIRONS DE GENÈVE. Genève, J. J. Paschoud, Imprimeur-Libraire, Paris, Mème maison de Commerce, 1820, p.i-xvi, 1-260, 22 plates. [Ostracod portion, the "Seconde Division, Monocles à coquille bivalve," p.159179, pls.17-19. Eighteen species of Monoculus $=$ Cypris of 0 . F. Müller, of which the species ruber, aurantius, monachus, virens, fuscatus, punctatus, striatus, villosus, ophtalmicus and ovum are new. Film, University of Michigan. 
Kaufmann, A. 1892. ÜBER DIE GATTUNG ACANTHOPUS VERNET UND EINE NEUE SÜSSWASSERCYTHERIDE. Zool. Anzeiger, Leipzig, vol.15, p.393-395. [Both species of Acanthopus belong to other genera; Leucocythere, n.gen. L. mirabilis, n.sp.] Film, University of Illinois.

- 1900a. ZUR SYSTEMATIK DER CYPRIDEN. Naturf. Gesell., Bern, Mitteilungen, p.103-109. [A brief, but major reclassification of fresh water ostracods:

Cyprididae; Notodromadinae, Herpetocypridinae, Cypridinae; Cypridopsinae, Cyclocypridinae, Ilyocypridinae, Candoninae, Pontocypridinae.] Film, University of Illinois. - 1900b. CYPRIDEN UND DARWINULIDEN DER SCHWEIZ. Revue Suisse Zool, Geneva, vol.8, p.209-423, pls.15-31. [Same classification as above paper, but in detail; with key for identification of genera and species, and extensive bibliography. Dolerocypris, Pseudocandona, n.gen.] Film, University of Illinois.

- 1900c. neUe ostracoden aUS der schweiz. Zool. Anseiger, vol. 23, p.131-133 [Cypridopsella, nov.nom. for Candonella Vavra, Paracypridonsis, Microcypris, Cryptocandona, n.gen., and $10 \mathrm{n.sp.]} \mathrm{Film,} \mathrm{L.S.U.}$ 
Kay, G. Marshall. 1934. MOHAWKIAN OSTRACODA: SPECIES COMMON TO TRENTON FAUNULES FROM THE HULL AND DECORAH FORMATIONS. Jour. Paleo., vol.8, no.3, p.328-343, pls.44-46. [Punctaparchites, Hallatia, Thomasatia, Bassleratia, Bellornatia and Raymondatia new genera and 11 new species]. Film, I.S.U.

- 1940a. ORDOVICIAN MOHAWKIAN OSTRACODA: LOWER TRENTON DECORAH FAUNA. Journ. Paleo., vol. 14, no.3, p.234-269, pls.29-34. [80 species of ostracoda, 28 new; Saccelatia, Winchellatia, Maratia, Parenthatia, Opikatia, and Bolbiprimitia new genera; Dilobellinae, new sub-fam. of Primitiidae]. Film, L.S.U.

- 1940b. DECORAH OSTRACODA, CORRECTION. JOUHM. Paleo., vol. 14, no.6, p.615. [Notes and genotype of Eridoconcha should be E. rugosa Ulrich and Bassler, and spelling of Saccelatia is corrected]. Film, I.S.U. Kegel, Wilhelm. 1932. ZUR KENNTNIS PALÄOZOISCHER OSTRAKODEN IT. BAIRDIIDAE AUS DEM MITTELDEVON DES RHEINISCHEN SCHIEFERGEBIRGES. Preuss. Geol. Landes., Jahrb., 1931, vol. 52, p.245-250, plate 13. [Bairdiocypris, n.subgen. of Bythocypris.] Film, L.S.U.

1933. ZUR KENNTNIS PALÄOZOISCHER OSTRAKDDEN 3. LEPERDITIIDAE AUS DEM MITTELDEVON DES RHEINISCHEN SCHIEFERGEBIRGES, Preuss. Geol. Landes., Jahrb., 1932, 


\section{- 1933. (Continued).}

vol. 53, p.907-935, p1.46. [Briartina, n.subgen. of Leperditia, and $10 \mathrm{n.sp.]} \mathrm{Film,} \mathrm{L.S.U.}$

- 1934. ZUR KENNTNIS PALÄOZOISCHER OSTRAKODEN. 4. ÜBER DIE GATTUNG ENTOMIS UND IHRE MITTELDEVONISCHEN ARTIN. Prouss. Geol. Landes., Jahrb 1933, vol.54, p.409-4+20, 10 text figs. [Herrmannina, n.name for Hermanne]]a, and $4 \mathrm{n}$.sp. of Entomis (Richteria); Richteria Jones $187 l_{4}$ revived as a subgenus of Entomis, with cypridina Serratostriata Sandberger 1845 designated subgenotypr. j Film, L.S.U.

kel1 tet, Betty. 1933. OSTRACODES OF THE UPFER PENNGYLVANTAN AND IHE LOWER PFRMIAN STRATA OF KANSAS: I. THE APARCHITIDAE, BEYRICHIIDAE, GIYPTOPLCURIDAE, KLOEDENELLIDAE, KIRKBYIDAE, AND YOUNGIELLIDAE. Jour. Paleo., vol.7, no.1, p.59.108, p1s. 13-16. [Pseudoparaparchites, Beyrichiana, Kniphtina, n.gen., and 21 new species; Youngiellidae, n.fam.] Film, L.S.U.

- 1935. OSTRACODES OF THE UPPER PENNSYLVANIAN AND LOWIR PERMIAN STRATA OF KAISAS: IIJ. BATRDIIDAE (CONCLUNED), CYTIERELLIDAE, CYPRTDINIDAE, ENTONOCONCHIDAE, CYTEERIDAE AND CYPRIDAE. Jour. Paleo., vol.9, no.2, p.132-166, pls. 16-18 ínryel1ina, Basslere11a, Haworthina, n.gen., and 16 n.sp.] Film, I.S.U. 
- 1936. CARBONIFEROUS OSTRACODES. Jour. Paleo.,

vol.10, no.8, p.769-784. [no figures]. [Graphio-

dactylidae, n.fam.; Antiparaparchites Coryell and Rogatz =

Paraparchites; Kegelites shown founded on young of

Amphissites dattonensis; Coryellites, nom.nov. for

Coryellina Kellett, not Bradfield; 32 species placed in synonymy of previously described ones, one new name, 3 new combinations, and 6 n.sp.] Film, L.S.U.

- 1943. PERMIAN OSTRACODES. Jour. Paleo., vol.

17, no.6, p.615-638. [A discussion of faunas and

environments with a checklist and bibliography]. Film, L.S.U.

Kertész, Koloman. 1893. DATEN ZUR OSTRACODEN-FAUNA DER

UMGEBUNG SZEGHALOM:S. Természetrajzi Füzetek, Budapest, vol.16, p.169-176, pl.6. [Ilyocypris gibba tuberculata, p.170, pl.6] Film, Iowa State College.

Kesling, Robert V. 1951. A NEW GENUS AND SPECTES OF PRIMITIOPSID OSTRACOD FROM THE DEVONIAN TRAVERSE GROUP OF MICHIGAN. Michigan Univ., Mus. Paleont., Contrib., vol.8, no.9, p.221, 230, 3 pls. [Sulcicuneus n.gen., S. porrectinatium n.sp.] Film, L.S.U. - 1951b. TERM INOLOGY OF OSTRACOd CARAPACES. Michigan Univ., Mus. Paleo., Contrib. vol.9, no.4, p. 93-171, 18 pls, 7 figs. 5 charts. L.S.U. 
1952a. OSTRACODS OF THE FAMILIES LEPERDITELIIDAE, PRIMITIIDAE, DREPANELLIDAE, AECHMINIDAE, AND KIRKBYIDAE, FROM THE MIDDLE DEVONIAN BELL SHALE OF MICHIGAN. Mich. Univ. Mus. Paleont., Contrib. vol.10, no.2, p.21-44, 5 pls. [New genera: Arcyzona, Sphenicibysis, Chironiptrum] Film, L.S.U.

- 1952b. DIMORPHISM IN DEVONIAN HOLLINID OSTRACODS OF NORTH AMERICA. Jour. Paleo., vol.26, no.5, p.764-771,

1 f'ig. [Abditoloculina n.gen., A. insolita n.sp.] Film, L.S.U.

- 1953a: OSTRACODS OF THE FAMILY DREPANELLIDAE FROM THE ARKONA SHALE OF ONTARIO. Michigan Univ., Mus. Paleo., Contrib., vol.10, no.8, p.193-202, 1 plate. [Tmemolophus, Tetrastorthynx, Xystinotus, n.gen., and 5 n.sp.] Film, L.S.U.

- 1953b. A NEW BEYRICHIID OSTRACOD FROM THE MIDDLE DEVONIAN ROCKPORT QUARRY LIMESTONE OF MICHIGAN. Michigan Univ., Mus. Paleo., Contrib., vol.10, no.10, p.221-229, 2 pls. [Phlyctiscapha n.gen., $\underline{P}$. rockportensis, n.sp.] Film, I.S.U.

- 1953c. OSTRACODA OF THE FAMILY AECHMINIDAE FROM THE ARKONA SHALE OF SOUTHERN ONTARIO. Michigan Univ., Mus. Paleont., Contrib., vol.1], no.1, p.1-10, 1 pl. [3 n.sp. Sigynus, n.gen.] Film, I.S.U. 
- 1953d. A BEYRICHIID OSTRACOD FROM THE MIDDLE

DEVONIAN WANAKAH SHALE. Buffalo Soc. Nat. Sci., Bul1., vol. 21, no.2, p.19-24, pl.9. [Hibbardia, n.gen.]

Film, University of Illinois.

1954. OSTRACODS FROM THE MIDDLE DEVONIAN DUNDEE

LIMESTONE IN NORTHWESTERN OHIO. Michigan Univ., Mus. Palea, Contrib., vol.1l, no.8, p.167-186, 3 pls.

[5 n.sp., Endolophia, Trypetera, n.gen.] Film, I.S.U.

Kesling, Robert V., and Copeland, Murray J. 1954. A NEW

KIRKBYID OSTRACOD FROM THE WANAKAH MEMBER OF THE MIDDLE DEVONIAN LUDLOWVILIE FORMATION IN WESTERN NEW YORK. Michigan Univ., Mus. Paleo., Contrib., vol.11, no.2, p.153-165, 2 pls.1, fig. [Amphizona, n.gen., A. asceta, n.sp.] Film, I.S.U.

Kesling, Robert V., and Hussey, Russell C. 1953. A NEW FAMILY AND GENUS OF OSTRACOD FROM THE ORDOVICIAN BILL'S CREEK SHALE OF MICHIGAN. Michigan Univ., Mus. Paleont., Contrib. vol.11, no.4, p.77-95, 2 pls., 1 fig. [Quadrijugatidae, n.fam., Quadrijugator, n.gen.] Film, L.S.U.

Kesling, Robert V., and McMillan, Gordon W. 1951. OSTRACODS OF THE FAMILY HOLLINIDAE FROM THE BELL SHALE OF MICHIGAN. Michigan Univ., Mus. Paleont., Contrib., vol.9, no.2, p.45-81, pls.1-7, figs, map. [15 n.sp., Proplectrum, Subligaculum, Falsipollex, n.gen.] Film, L.S.U. 
Kesling, Robert V., and Weiss, Martin. 1953. OSTRACODA FROM THE NORWAY POINT FORMATION OF MICHIGAN. Michigan Univ., Mus. Paleont., Contrib. vol.11, no.3, p.33-76, 5 pls. [17 n.sp.; Ruptivelum, Reticestus, n.gen.] Film, L.S.U.

King, R. L. 1855. ON AUSTRALIAN ENTOMOSTRACANS. ROYaI SOc. Tasmania [Van Diemen's Land] vol.3, pt.1, p.56-75, pls.9,10 [Cypris, $10 \mathrm{n} . \mathrm{sp} .$, Candona, $1 \mathrm{n.sp}$. ; Newnhamia fenestrata n.gen., n.sp.] Film, Yale University•

Kingma, J. T. 1948. CONTRIBUTIONS TO THE KNOWLEDGE OF THE YOUNG-CAENOZOIC OSTRACODA FROM THE MALAYAN REGION. Published in Utrecht, $118 \mathrm{pp} ., 11 \mathrm{pls}$. and distribution chart. [Hemicytheridea, Atjehella, Paijenborchella, Javanella, Neomonoceratina, Tannella, n.gen.; and 4 I n.sp.] Film, L.S.U. from Morton Stephenson's copy.

Kirkby, J. W. [and Jones, T. R.] 1860. ON PERMIAN ENTOMOSTRACA FROM THE SHELL-LIMESTONE OF DURHAM. By. J. W. Kirkby. WITH NOTES ON THE SPECIES BY T. Rupert Jones, F.G.S. Tyneside Nat. Field Club, Trans. vol.2, 1858-1860, 1860, p.122-171, pls.8-11. [Kirkbya Jones, n.gen. p.129] Film from Harvard.

Klie, Walter. 1929. BEITRAG ZUR KENNTNIS DER OSTRACODEN DER SÜDLICHEN UND WESTLICHEN OSTSEE, DER FESTLÄNDISCHEN NORDSEEKÜSTE UND DER INSEL HELGOLAND. Zeitschrift fur 
Klie, Walter. 1929. (Continued).

Wissenschaftliche Zoologie, Leipzig, vol.132, p.270306, 34 Abbildungen. PParacyprideis, n.gen., and 6 n.sp. of various genera]. Film, University of Michigan.

- 1931. CAMPAGNE SPÉOLOGIQUE DE C. BOLIVAR ET R. JEANNEL DANS L'AMÉRIQUE DU NORD (1928). 3. CRUSTACÉS OSTRACODES. Archives de Zool. Expér. et Générale, Paris, Tome 7l, p.333-344, 20 figs. [3 n.sp.] Film, University of Illinois.

1932. DIE OSTRACODEN DER DEUTSCHEN LIMNOLOGISCHEN SUNDAEXPEDITION. Archiv für Hydrobiol. Stuttgart. Suppl. Bd. 11, p.447-502, pls.64-69. [13 n.sp. Pseudocypretta, n.gen.] Film, University of Illinois.

- 1935. OSTRACODA AUS DEM TROPISCHEN WESTAFRIKA. In: VOYAGE DE CH. ALLUAUD ET P.A. CHAPPUIS EN AFRIQUE OCCIDENTALE FRANCAISE (1930-1931). Archiv. Hydrobiol., Stuttgart, vol.28, p.35-68, 63 figs. [Strandesia parva, Stenocypris chappuisi, S. caesia, Cypridopsis alluaudi, C. minima, Zonocypris, laevigata, Darwinula serricaudata, D. africana, D. inconspicua, n.spp•; Afrocythere n•gen., A. rostrata, n.sp.] Film, University of Illinois.

1935b. SÜSSWASSER-OSTRACODEN AUS URUGUAY. Archiv Hydrobiol., Stuttgart, vol.29, p.282-295, 26 figs. [4 n.sp.; Riocypris, n.gen.] Film, University of Illinois. 
1936a. ZUR KENNTNIS DER OSTRACODENFAMILIE

POLYCOPIDAE. Zool. Jahrbücher, Jena, Abt. Syst. vol. 68, p.517-532, 27 figs. [3 n.sp. Parapolycope, n.gen.] Film, University of Illinois.

- 1936b. OSTRACODEN DER FAMILIE CYTHERIDAE AUS SAND UND SCHELI VON HELGOLAND. Kieler Meeresforschungen, Kiel. Bd.1, p.59-72, 50 figs. [8 n.sp., Psammocythere, n.gen.] Film, University of Illinois.

- 1938a. KREBSTIERE ODER CRUSTACEA III: OSTRACODA, MUSCHELKREBSE. in Dahl's Die Tierwelt Deutschlands und der angrenzenden Meeresteilt, 34 Teil, III, pp. ivt230, 786 figs. [A text book of German ostracods, new subfamilies Psammocytherinae and Microcytherinae.] Film, University of Illinois.

- 1938b. OSTRACODEN AUS UNTERIRDISCHEN GEWÄSSERN

IN SÜDITALIEN. Zool. Anzeiger, vol.123, p.148-155,

16 figs. [Pseudolimnocythere, n.gen., 2 n.sp.] Film, University of IIIinois.

- 1939a. OSTRACODEN AUS DEM KENIA-GEBIET, VORNEHMLICH VON DESSEN HOCHGEBIRGEN. Internat. Rev • Hydrobiol u Hydrog., Leipzig, vol.39, p.99-161, 79 figs. [Globocypris, n.gen., and $14 \mathrm{n} . \mathrm{sp}$. assigned to 12 genera]. Film, University of Illinois. 
- 1939b. BRACKWASSEROSTRACODEN VON NORDOSTBRASILIEN.

Zool. Jahrb. Abt. f. Syst., Jena, vol.72, p.359-372, 10

figs. [Ilyocythere, n.gen., I. gibba, I. cribrosa n.sp.]

Film, University of Illinois.

- 1939-1940. SÜSSWASSEROSTRACODEN AUS NORDBRASILIEN. Zool. Anseiger, Leinzig, vol.128, 1939, I. 0.84-91, 12 figs; II. DIE GATTUNG CHLAMYDOTHECA, p.152-159, 13 figs; III. DIE GATTUNG STENOCYPRIS UND DOLEROCYPRIS, p.316-320, 3 figs; vol. 12́9, 1940; IV. EUCYPRIS ODER HETEROCYPRIS?, P.113-120, 10 figs.; V. DIE GATTUNG STRANDESIA, p.201-206, 8 figs; vol.130, 1940, VI. CYPRINAE MIT GEISSELFÖRMIGER FURKA, p.59-73, 7 figs.; VII. ILYOCYPRINAE UND CANDOCYPRINAE, p.219-229, 19 figs.; VIII. DARWINULIDAE UND CYTHERIDAE. RÜCKBLICK, p.300303. [Pelocypris, Neocypridopsis; 12 n.sp. assigned to 9 different, genera, as well as many previously described species.] Film, L.S.U.

- 1940. BEITRäGE ZUR FAUNA DER EULOTORALS VON DEUTSCH-SÜDWESTAFRIKA II. OSTRACODEN VON DER KÜSTE DEUTSCH-SÜDWESTAFRIKAS. Kieler meeresforschungen, Bd. III, Heft $2,0.404-448,91$ figs. [Eucythereis, n.gen., 17 n.species]. Film, University of California, Feb. 1954. KIöden, K. F. 1834. DIE VERSTEINERUNGEN DER MARK BRANDENBURG, INSONDERHEIT DIEJENIGEN, WELCHE SICH IN DEN ROLLSTEINEN UND BLÖCKEN DER SÜDBALTISCHLN EBBEN FINDEN. Berlin, 378 pp , 10 pls 
KIöden, K. F. 1834 (Continued).

[Crustacea, p.101-122; Cytherina phaseolus Hising., p.102; Battus tuberculatus n.sp., p.112, pl.1, f.16-23.]

Film of Yale copy.

Knight, J. Brookes. 1928. SOME PENNSYLVANIAN OSTRACODES FROM THE HENRIETTA FORMATION. OF EASTERN MISSOURI. Part I. Jour. Paleo. vol.2, no.3, p.229-267, pls.30-34; Part II. Jour. Paleo. vol.2, no.4, p.318-336, pls.43, 44. Film, I.S.U.

Koch, Carl Ludwig. 1835-1844. DEUTSCHLANDS CRUSTACEEN, MYRIAPODEN UND ARACHNIDEN. EIN BEITRAG ZUR DEUTSCHEN FAUNA. Herausgegeben von Herrich Schäffer, Regensberg, F. Pustet. Heft 10, 1837, 1.ypris tricinata, 2.‥ maculata, 3.‥ variabilis, $4 . \underline{C}$. leucomela, 5. $\underline{\mathrm{C}}$. brunnea, 6.C.lepidula. Heft 11, 1837, I.Cypris monacha O.F.M.,

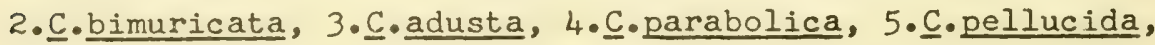
6.‥pubescens. Heft 12, 1837, 1-2. Cypris epihippiata, 3. . tenera, 4. C.nubilosa. Heft 21, 1938, 12-14. Cypris conchacea Desmerest, 15. C..Iutraria, 16. $\underline{\text {. }}$

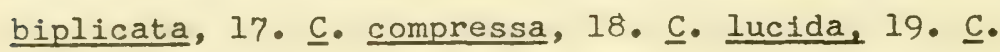
galbinea, 20. ‥ gibberula, 2I. C.fuscata Desm. 22. C. serena, 23. C. punctata Desm. 24. C. villosa. Heft 36 , 1841, 17. Cypris ophthalma Desmarest, 18. C. laevis O.F.M., 19. C. strigata O.F.M. Title page of each Heft lacks date of publication. Species, rather than pages are numbered. Film from Library of Congress. 
Koninck, L. De. 1841. MÉMOIRE SUR LES CRUSTACÉS FOSSILES DE BELGIQUE. Acad. Roy. Sci., Lettres, Beaux-Arts de Belgique, Brussels, Mém. 14, p.1-20, and plate.

[Famille des Cyproides; Cytherina Lamarck; Cypridina Milne Edwards, Cyprella, nov. Cypridella nov., and 6 n.sp.] Krause, Aurel. 1889a. UEBER BEYRICHIEN UND VERWANDTE OSTRACODEN IN UNTERSILURISCHEN GESCHIEBEN. Deutsch. geol. Gesell., Zeitschr. vol.41, p.1-26, pls.1-2. [9 n.sp. of Primitia; 2 Entomis; 2 Bollia; 2 Strepula; 4 Beyrichia; 1 Kloedenia.] Film, L.S.U.

- 1892. NEUE OSTRAKODEN AUS MÄRKISCHEN SILURGESCHIEBEN. Deutsche Geol. Gesell. Berlin, Zeitschr. vol.44, p.383399, pls.2l-22. [30 n.sp. and vars.] Film, L.S.U.

Krommelbein, Karl. 1952. DIE TAXONOMISCHE STELLUNG DER GATTUNG BAIRDIOCYPRIS UND IHRE ARTEN IM MITTEL-DEVON. Senckenbergiana, Bd. 32, no.5-6, p.319-335, pls.1-4. [Bairdiocypris placed in Healdiidae, 6 species, two new]. Film, I.S.U.

Kummerow, E. 1924. BEITRÄGE ZUR KENNTNIS DER OSTRACODEN UND PHYILOCARIDEN AUS NORDISCHEN DILUVIALGESCHIEBEN. PreusS. Geol. Landes., Jahrb. 1923 [1924], Bd. 44, p.405-443, pls. 21-22. [Craspedobolbina n.gen., 86 sp., of which 44 are new]. Film, L.S.U. 
1931. ÜBER DIE UNTERSCHIEDE ZWISCHEN PHYLLOCARIDEN UND OSTRACODEN. Centralblatt f.Min.Geol.Pal.,Jahr 1931, Abt.B., no.5, p.242-257, 18 text figs. [Aparchites anderssoni, A? fennicus, Beyrichona gecalensis, B. faba, B. alta, Sellula fallax, Bradorona nitida, Hipponicharion mathewi of Wiman; Indiana dermatoides, Aristozoe rotundata of Walcott. A. primordialis Linn., Leperditia? lentiformis Cobbold, Polyphyma lapworthi Groom, P. angelini Barrande, P. angelini armata Grönwall, and Aristozoe? minima, n.sp. assigned to Phyllocardia.] Film, L.S.U.

- 1933. ZUR PALAOBIOLOGIE DER OSTRAKODEN UND TRILOBITEN. Centralblatt f. Min. Geol. Pal. Jahr. 1933, Abt.B., no.1, p.42-53, 12 figs. [Platychilina, n.gen.] Film, I.S.U.

1939. DIE OSTRAKODEN UND PHYLLOPODEN DES DEUTSCHEN UNTERKARBONS. Preuss. Geol. Landesanstalt, Abh., N.F., Heft 194, 107 pp., 7 pls., so figs. [Leioprimitia, Platychilina, Entoprimitia, Kirkbyellina, n.gen., and 38 new species belonging to 26 genera]. Film, L.S.U.

- 1943. DIE OSTRAKODEN DES GRAPTOLITHENGESTEINS. Zeitschrift fur Geschiebeforschung, Bd. 19, H.1, p.2760, pls.1,2. [6 new species; Opisthoplax, n.gen.] Film, L.S.U. 
- 1949. ÜBER EINIGE SÜSSWASSER-OSTRACODEN DES

RUHRKOHLENGEBIETES. Neues Jahrb., Monatshefte, Abt.B, p.45-49, I fig., 2 pls. [Limnoprimitia, n.gen., L. arcuata (Bean), I. robusta, n.sp.]. Film, L.S.U.

- 1953. ÜBER OBERKARBONISCHE UND DEVONISCHE OSTRACODEN IN DEUTSCHLAND UND IN DER VOLKSREPUBLIK POLEN. Geologie, Beiheft zur Zeitschrift, Nr. 7, p.1-75, pls.1-7, Berlin. [Leptoprimitia, Ctenobolbinella, Acrossula, Thlipsurina, Orthocypris, Omphalentomis, n.gen., and 85 new species; Limnoprimitia redescribed as new, p.15.] Film, L.S.U. Lamarck, le Chevalier de. 1818. HISTOIRE NATURELLE DES ANIMAUX SANS VERTÈBRES. Ist. edit. Paris, vol.5 [Crustacea p.109t; Cypris, 3 sp. p.123; Cytherina 2 sp.p.125]. Film of Yale copy.

Latham, Mary H. 1932, 1933. SCOTTISH CARBONIFEROUS OSTRACODA. Royal Soc. Edinburgh, Trans., vol.LVII, Pt.2, Feb.4, 1933, p.351-395, 25 text figs. (issued separately October 1932) [ Tribolbina, n.gen., T. carnegei, Hollina avonensis, n.sp.] Film, L.S.U.

Latreille, P. A. 1802 [AN.X]. HISTOIRE NATURELLE, GÉNÉRALE ET PARTICULIÈRE DES CRUSTACÉS ET DES INSECTES. Ouvrage faisant suite à l'Histoire Naturelle générale et particulière, composée par Leclerc de Buffon, et rédigée par C.S.Sonnini, membre de plusieurs Sociétés savantes. 
Latreille, P. A. $1802[\mathrm{AN} \cdot \mathrm{X}]$. (Continued)

Paris, 1802, 8 vo. [Ostrachodes; ostrachoda, vol.3,

p.17. Cypris, vol.4, p.232-240; C. delecta, C. ornata, C.

laevis, $\underline{\text {. fasciata, }} \underline{\text { C. strigata, }}$. vidua, $\underline{C}$. conchacea,

C. pilosa, C. monacha, C. crassa, C. candida, p.241-248.

Cythere, p.249-25I, C. viridis, C. Iutea, ‥ flavida,

C. gibba, ‥ gibbera, p.252-254]. Film, I.S.U.

- 1806. GENERA CRUSTACEORUM ET INSECTORUM,

Tomus I, 1806, p.I-XVIII, 1-303 [Tomus II, 1807, p.1-280].

[Ostracoda, Tomus I, p.9, 17, with genera Lynceus, Daphnia,

Cypris, and Cythere. Film of Ohio State University copy.

- 1810. CONSIDÉRATIONS GÉNÉRALES SUR L'ORDRE

NATUREL DES ANTMAUX COMPOSANT IES CLASSES DES CRUSTACÉS,

DES ARACHNIDES, ET DES INSECTES, AVEC UN TABLEAU MÉTHODIQUE

DE LEURS GENRES, DISPOSÉS EN FAMILLES. Paris. [Seconde

Partie, p.88; Entomostracés, Entomostraca; Famille I.

Clypéacés, Aspidiota; Famille II. Ostracodes, Ostracoda,

p.89-91. Filmed from University of Pennsylvania copy •

- 1831. COURS D'ENTOMOLOGIE, OU DE L'HISTOIRE

NATURELLE DES CRUSTACÉS, DES ARACHNIDES, DES MYRIAPODES

ET DES INSECTES, A L'USAGE DES ÉLÉVES DE L'ECOLE DU MUSÉUM

D'HISTOIRE NATURELLE. Paris. [Huitième Ordre: Ostrapodes

(Ostrapnda), p.429-432]. Filmed from Harvard University copy 
LeROy, L. W. 1939. SOME SMALL FORAMINIFERA, OSTRACODA AND OTOLITHS FROM THE NEOGENE ("MIOCENE") OF THE ROKAN-TAPANOELI AREA, CENTRAL SUMATRA. Natuur. Tijdschrift v. Neder-Indië, AfI. 6 van Deel XCIX, p.215-296, pls. 1-14 [Ostracods p.272-277, pls.10-12. Thalmannia n.gen.,

I. sumatrensis, Cytherella telisaensis, Cytherella truncata, Cythereis (Pterygocythereis?) telisaensis, C. holmani, n.spp.] Film, I.S.U. of my copy•

Levinson, Stuart. 1951. THIN SECTIONS OF PALEO7.OIC OSTRACODA AND THEIR BEARING ON TAXONOMY AND MORPHOLOGY. JOUr. Paleo., vol.25, no.5, p.553-560, pl.77. [Cryptophyllus n.gen. and others discussed.] Film, I.S.U. - 1953. BIBLIOGRAPHY AND INDEX TO NEW GENERA OF OSTRACODA, 1950-1952. The Micropaleontologist, vol.7, p.51-64. Film, L.S.U.

Lienenklaus, E. 1894. MONOGRAPHIE DER OSTRAKODEN DES NORDWESTDEUTSCHEN TERTIÄRS. Deutsche Geol. Gesell., Berlin, Zeitschr., vol. 46, p.158-268, pls.13-18. [47 n.sp. and vars. and Cuneocythere, n.gen.] Film, L.S.U.

Liljeborg, W. 1853. [spelled Lillieborg in later papers] DE CRUSTACES EX ORDINIBUS TRIBUS: CLADOCERA, OSTRACODA, ET COPEPODA, IN SCANIA OCCURRENTIBUS. OM DE INOM SKANE FÖREKOMMANDE CRUSTACEER AF ORDNINGERNE CLADOCERA, OSTRACODA OCH COPEPODA. Lund 1853, xvi+222pp., 27 pls. [Ostracoda: p.92-130,164-177, pls.8-12,17-19,25-26. Notodromas and Philomedes, n.gen.] Film, Crear Lib. 
- 1883. COLLECTION OF CHIEFLY FRESHWATER CRUSTACEA

FROM SWEDEN in Creat International Fisheries Exhibition, London, 1883, Sweden Spccial Catalogue, p.140-150.

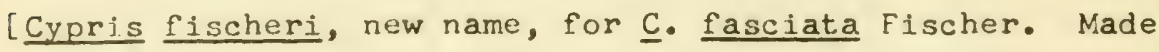
genotype of Stenocypria by G. W. Muller 1901b]. Film, U.S. Dept. Interior Library.

Linnaeus, C. [Linné, C., or Caroli Linnaei]. 1758. SYSTEMA NATURAE PER REGNA TRIA NATURAE, SECUNDUM CLASSES, ORDINES, GENERA, SPECIES, CUM CHARACTERIIBUS, DIFFERENTIIS, SYNONYMIS, LOCIS. Tomus I. Editio Decima, [?Ostracods: Monoculus, M. polyphemus, M. foliaceus, M. apus, M. pulex, M. pediculus, M. quadricornis, M. conchaceus, M. Ienticularis, M. Telemus, p. 634,635 . Of these M. conchaceus has been thought by some authors to be an ostracod; . Ienticularis and $\underline{M}$. telemus are considered by Skogsberg 1920, p.151 to also be presumably ostracods]. Film, L.S.U.

Iinnarsson, J.G.0. 1869. OM VESTERGÖTLANDS CAMBRISKA OCH SILURISKA AFLAGRINGAR. Svenska Vetenskapsakademiens, Stockholm, Handlingar, Bd. 8, no. 2. [Ostracods p. 84-88, pl.2. Leperditia primordialis, Beyrichia costata, n.spp.].

Liventhal, B. E. 1929. Лнвентвь B. Э. Ostracoda ануагыльсого и апшерон сного ярусов по Бабазанан сному разрөзу. Из раоот исслөдов. Лвоорат. по геологид нефтн. Изд. Изв. Аз ербаилж. полит. ин-та, Баку, $19 \%$. [Not seen; but Schweyer 1936, p.21 indicates that this paper contains Bairdia, 3 sp., Crthere 9 sp., Limnicythere, 2 sp., Loxoconcha $4 \mathrm{sp}$, Candona $8 \mathrm{sp.,}$ mostly described as new.] 
Loetterle, Gerald J. 1937. THE MICROPALEONTOLOGY OF THE NIOBRARA FORMATION IN KANSAS, NEBRASKA, AND SOUTH DAKOTA. Nebraska Geol. Survey Bull. no.12, 73pp., Il pls. [Ostracods, p.49-56, 64, 65, pls. 8, 9, 11. Morrowina, n.gen.] Film, I.S.U.

Lowndes, A.G. 1931. SOME RARE AND LITTLE-KNOWN BRITISH FRESH-WATER OSTRACODS. Marlborough College, Marlborough, England, Nat.Hist.Soc., Rept., no.79, p.82-112, 87 figs., 2 pls. [a redescription of Pseudocandona and its type species $\underline{P}$. pubescens $(K o c h)]$. Film, University of Illinois. - 1932. REPORT ON THE OSTRACODA. MR. OMER COOPER'S INVESTIGATION OF THE ABYSSINIAN FRESH-WATERS (DR. HUGH SCOTT'S EXPEDITION). Zool. Soc. London, Proc. 1932, p.677-708, 34 text figs. [Thirteen species, mostly new.] Film, L.S.U.

Lutkevich, E. M. 1939. ORDER PHYLLOPODA. In Atlas of the Leading Forms of the Fossil Fauna USSR., vol.6, Permian, p.190-193, pl.XLVI. The Central Geological and Prospecting Institute, Moscow. [Vertexia, Cornia Lutkevich, p.192; both genera having been referred apparently errouneously to the ostracoda by Agnew 1944, p.218, 219]. Film of Stanford copy. 
M'Coy, Frederick. 1839. ON A NEW GENUS OF ENTOMOSTRACA. Royal Geological Society of Ireland, Dublin, Journal, vol.2, p.9]-94, plate 5. [Entomoconchus scouleri, n.gen., n.sp.] Film, University of Michigan.

1844. A SYNOPSIS OF THE CHARACTERS OF THE CARBONIFEROUS LIMESTONE FOSSILS OF IRELAND. DubIin, University Press, 207pp., 29 pls. [Crustacea p.159-168, p1.23.; Bairdia n.gen., p.164.] Reissued by Sr. Richard Griffith in 1860. Film, L.S.U. from Harvard Mus. Comp. Zool. copy.

- [MCCOY]. 1846. A SYNOPSIS OF THE SILURIAN FOSSILS OF IRELAND, COLLECTED FROM THE SEVERAL DISTRICTS BY RICHARD GRIFFITH, F.G.S., THE WHOLE REING NAMED AND THE NEW SPECIES DRAWN AND DESCRIBED PY FREDERICK M'COY, F.G.S.D. Dublin, 68 pp., 5 pls. [Entomostraca, p.57-58, Beyrichia, n.gen.] Film made L.S.U. from Pinceton copy. - 1849. ON THE CLASSIFICATION OF SOME BRITISH FOSSIL CRUSTACEA, WITH NOTICES OF NEW FORMS IN - THE UNIVERSITY COLLECTION AT CAMBRIDGE. Ann. Mag. Nat. Hist., ser.2, vol.4, p.161-179, 330-355, 392-414. [Cytheropsis, n.gen., p.414]. Film, L.S.U. - 1851. ON SOME NEW CAMBRO-SILURIAN FOSSILS. . Ann. Mag. Nat. Hist., ser.2, vol.8, p.387-409. [Cytheropsis aldensis MiCoy only ostracod described]. Film, I..S.U. 
Malkin, Doris S. 1953. BIOSTRATIGRAPHIC STJDY OF MIOCEME OSTRACODA OF NEW JERSEY, MARYI.AND, AND VIRGINTA. JOUY. Paleo., vol.27, no.6, p.761-779, pls.75-82, 14 figs. Film, I.S.U.

Marshall, W. S. 1903. ENTOCYTHERE CAMBARIA (XOV.GEY.ET. NOV.SPEC.), A PARASITIC OSTRACOD. Wisconsin Acad. Sci. Arts and Letters, Trans., vol.14, pt.1, p.117-144, p1s. 10-13. Film, L.S.U.

Martin, Gerald R.P. 1940. OSTRACODEN DES MORDDIUTSCHEA PURBECK UND WEALDEN. Senckenbergiana, vol.22, p.275361, pls.1-13. [l1 n.sp. of Cypridea, I of klieana, I of Macrodentina, 2 of Orthonotacythere, 3 of Gomphosvthere, 1 of Iimnocythere, 1 of Cyprideis, and $2 \mathrm{n} . \mathrm{subsp}$ of Ilyocypris. Cyprideinae, n.subfam., Klieana and llacrodentia, Film, L.S.U.

Masi, L. 1906a. CONTRIBITO ALLA SISTFMATICA DELLE "ILYCCYPRIUAL". Soc. Zool. Italiana, Rome, Bolletino, Ser. II, vol. VI [VII-VIII] p.133-146; 249-268. [Subfam. Ilyociprinae, Ilyocyprois, n.gen.] Film made at L.S.U. from copy borrowed from Rice.

[1925-26]. 1928. DESCRIZIONE DI TRE NUOVI OSTRACODI AFRICANI. Genoa. Museo Civ. Stor. Nat., Annali, vol.52, 1925-26 [1928 fide Zoological Rucord], p.50-63. [Cyprinotoides n.subgen. of Cyprinotus, p.51, and $3 \mathrm{n} . \mathrm{sp}$. of ostracods]. Film, University of Illinois. 
Matern, Hans. 1929. DIE OSTRACODEN DES OBERDEVONS. I.TEIL. APARCHITIDAE, PRIMITIIDAE, ZYGOBOLBIDAE, BEYRICHIIDAE, KLOEDENELLIDAE, ENTOMIDAE. Preuss. Geol. Landes., Abhandlungen, N.F., Heft 118, p.1-99, pls.1-5. [Neochilina, Tetrasulcata, n.gen., Nehdentomis, Fossirichterina, n. subgen.] Film, L.S.U.

Matthew, G. F. 2886. ILLUSTRATIONS OF THE FAUNA OF THE ST. JOHN GROUP CONTINUED. NO.III. DESCRIPTIONS OF NEW GENERA AND SPECIES (INCLUDING A DESCRIPTION OF A NEW SPECIES OF SOLENOPLEURA BY J. F. WHITEAVES). ROy.Soc.Canada, Proc. and Trans. for 1885, Ser.1, vol.3, sec.4, p.29-84. [Lepiditta, Beyrichona, Leoidilla, Hipponicharion, n.gen., Beyrichinae n.subfam.] Film, L.S.U.

- 1896. FAUNAS OF THE PARADOXIDES BEDS IN EASTERN NORTH AMLRICA. NO.1. New York Acad. Sci., Trans., vol.15. p.192-247, 4 pls., I flg. [Aluta n.gen., A. flexilis, n.sp.] - 1899. PRELIMINARY NOTICE OF THE ÉTCHEMINIAN FAUNA OF CAPE BRETON. Nat.Hist.Soc. New Brunswick, BuI1., vol.4, pt.3, no.18, p.198-208. 'Bradoria n.gen., and new apecies of Bradoria and Schmidtella]. - 1902. OSTRACODA OF THE BASAL CAMBRIAN ROCKS IN CAP Li BRETON. Canadian Rec. Sci., Montreal, vol.8,p.437-468, pls. 1,2. [Bradorona, Escasona, Indiana, n.gen., or subgen.] Film, University of Illinois. 
Méhes, Gyula. 1911. ÜBER TRIAS-OSTRAKODEN AUS DEM BAKONY. Magyar Földrajzi Társaság, Budapest, Balaton-bizottsag, Resultate der wissenschaftichen erforschung des Balatonsees. Band 1, Thl.1, Part 3, Section 6, p.1-4. [26 n.sp., Hungarella, n.gen.] Film, Library of Congress. - 1941a. BUDAPEST KORNYÉKÉNEK FELSÖOLIGOCÉN OSTRACODÁI. [DIE OSTRACODEN DES OBEROLIGOZÄNS DER UMGEBUNG VON BUDAPEST. Geologica Hungarica, Ser. Pal., fasc. 16, $95 \mathrm{pp} ., 7 \mathrm{pls}$. and 143 text figs. [Pseudokrithe, Prionocytheretta, Budaia, n.gen. and 30 new sp. and vars.] Magyar with German summary. Film, University of California.

Miller, S. A. 1874. MONOGRAPH OF THE LAMELIIBRANCHIATA OF THE CINCINNATI GROUP. Cincinnati Quart. Journ. Sci., vol.1, no.3, p.21l-236, figs. [Beyrichia duryi, B. striato-marginata, B. chambersi, n.spp.] Film, L.S.U.

- 1875. SOME NEW SPECIES OF FOSSILS FROM THE CINCINNATI GROUP AND REMARKS UPON SOME DESCRIBED FORMS. Cincinnati Quart. Journ. Sci., vol.2, no.4, p.349-355. [Beyrichia cincinnatiensis, n.sp.] Film, L.S.U. - 1889. NORTH ANERICAN GEOLOGY AND PALEONTOLOGY FOR THE USE OF AMATEURS, STUDENTS, AND SCIENTISTS. Cincinnati, 664pp., illus. [Crustacea, p.522-569. Faberia, n.gen.] Film, I.S.U. 
1892, 1897. NORTH AMERICAN GEOLOGY AND

PALEONTOLOGY. Appendix I, 1892, Crustacea, p. 704-718. Appendix II. 1897, Crustacea, p.786-789. Film, Library of Congress.

Milne-Edwards, Henri. 1840. HISTOIRE NATURELLE DES CRUSTACÉS, etc. [faisant suite de Buffon] Tome troisième [III] Paris. 1834-1840 8 vo. [Ordre des Cyproîdes ou des Ostracodes, p.393-410, Cypridina, n.gen. p.409, C. reynaudi, n.sp. p.410, plate 36 ] Film, Library of Congress.

Moberg, Joh. Chr. 1895. SILURISK POSIDONOMYASKIFFER AN EGENDOMLIG UTBILDNING AF SKANES ÖFVERSILUR. Sveriges Geol. Unders., ser. C, no. 156, 21 pp., 1 pl. Stockholm. [Eoconchoecia, Colpos, $\mathrm{n}$. gen., and $5 \mathrm{n.sp.].} \mathrm{Film,}$ Library of Congress.

Moberg, J. C., and Segerberg, C. 0. 1906. BIDRAG TILL KÄNNEDOMEN OM CERATOPYGEREIGIONEN MED SÄRSKILD HÄNSYN TILL DESS UTVECKLING I FOGELSANGSTRAKTEN. Lund Universitets Åsskrift, N.F. Afd.2, Band 2, Nr.7, p.1-116, 7 pls. [Ostracods p.75, 76, pl.3. Eremos, n.gen. and $3 \mathrm{n}$. species: Beyrichia manella p.76 = genotype of Nanopsis Henningsmoen 1954a, p.54] Film, University of Calif., Feb. 1954 complete. 
Moore, Charles. 1867. ON ABNORMAL CONDITIONS OF SECONDARY DEPOSITS WHEN CONNECTED WITH THE SOMERSETSHIRE AND SOUTH WALES COAL-BASIN: AND ON THE AGE OF THE SUTTON AND SOUTHERNDOWN SERIES. Geol. Soc. London, Quart. Journ., vol. 23, p.449-568, pls.14-17 [Ostracod lists $\mathrm{p} \cdot 465,494,495,497-499,524-525,559$, with a number of nude names. No species described]. Film, L.S.U.

Moore, R. C. 1929. BASSLERINA, A NEW HOLLINIFORM OSTRACODE GENUS, WITH DESCRIPTION OF NEW PENNSYLVANIAN SPECIES FROM TEXAS AND OKLAHOMA. Denison Univ., Bull., Jour. Sci. Lab. vol. 24, p.99-113, pls. 6-8. Film, L.S.U.

Moniez, R. 1891. ENTOMOSTRACÉS D'EAU DOUCE DE SUMATRA ET DE CELEBES. II. OSTRACODES. in Dr. Max Weber: Zoologische Ergebnisse einer Reise in Niederländisch Ost-Indien, vol. 2, heft 1, p. 129-135, plate X. [Cypris weberi, . richardi, $\underline{\text { C. odiosa, }}$ C. sarsi, $\underline{\text { c. longiseta; Cyprinotus }}$ pyxidata, n.spp., Stenocypris malcolmsoni Brady]. Film, L.S.U. of copy from Museum of Zoology, Univ. Michigan.

Moore, R. C., Weller, J. M. and Knight, J. F. 1942. ERRONEOUS EMENDATION OF GENERIC NAMES. Jour. Paleo., vol. 16, no.2, p.250-261. [Graphiodactyllis vs. Graphiodactylus, p.256.] Film, L.S.U. 
Morey, P. S. 1935a. OSTRACODA FROM THE BASAL MISSISSIPPIAN SANDSTONE IN CENTRAL MISSOURI. Jour. Paleo., vol.9, no.4, p.316-326, pl.28. [Plagionephrodes and Hypotetragona, n.gen., with 23 new species of ostracods]. Film, I..S.U. - 1935b. OSTRACODA FROM THE AMSDEN FORMATION OF WYOMING. Jour. Paleo., vol.9, no.4, p.474-482, pl.54. [Balantoides, n.gen., and 16 new species of ostracods]. Film, L.S.U.

Morris, R. W., and Hill, B. L. 1951. SHIDELERITES, A NEW SILURIAN OSTRACODE GENUS. Journ. Paleo. vol.25, no.5, p.698, 699. Film, L.S.U.

- 1952. NEW OSTRACODA FROM THE MIDDIE SILURIAN NEWSOM SHALE OF TENNESSEE. Bull. Amer. Paleont., vol.34, no.112, p.131-148, pls.9,10. [Hemiaechminoides, Thlipsuroides, Spinobairdia, Newsomites, Pseudocyproides, n.gen. Daleiella redefined, and 7 new species]. Film, L.S.U.

Müller, Fritz. 1880. WASSERTHIERE IN BAUMWIPFELT • ELPIDIUM BROMELIARIM. Kosmos (Polskie Towarzystwo Przyrodników Imienia Kopernika) Lemberg, vol.6, p.386-388, text figs. 1-15. [Elpidium n.gen., E. bromeliarum n.sp.] Film, Univ. of California. 
- 1881. DESCRIPÇÃO DO ELPIDIUM BROMELIARUM

CRUSTACEO DA FAMILIA DOS CYTHERIDEOS. Rio de Janeiro, Museu Nacional, Archivos vol.4, p.27-34, pl.2. Film. University of Illinois.

Müller, G. W. 1884. ZUR NÄHEREN KENNTNISS DER CYTHERIDEN. Archiv. f. naturgeschichte, Berlin, vol.1, p.1-18, pls.1,2. [Cytherois, C. virens, n.gen., n.sp.]. Film, University of Illinois.

- 1890a. NEUE CYPRIDINIDEN, Zool. Jahrb., Abt. f syst., vol.5, p.211-252, pls.25-27. [Pyrocypris, n. gen, and 6 species; Philomedes, 2 species; Asterope, 3 species]. Film, University of Illinois.

1890b. UEBER HALOCYPRIDEN. Zool. Jahrbüch, vol.5, p.253-280, pls.28-29. Euconchoecia, n.gen; 1 n. sp. Halocypris, 6 of Conchoecia, and 1 of Euconchoecia.] Film, University of Illinois.

- 1894. DIE OSTRACODEN DES GOLFES VON NEAPEL UND DER ANGRENZENDEN MEERES-ABSCHNITTE. Naples. Sta. Zool•, Fauna und Flora des Golfes von Neapel, 21 Monographie, p.i-viii, 1-404, pls.1-40. [165 n.sp. assigned to 38 genera, the following new: Pseudophilomedes; Archiconchoecia; Polycopsis; Pontocypria; Erythrocypris; Paracythere, Eucytherura, Paracytherois, Nicrocythere, Microxestoleberis, 
- 1894. (Continued).

Paracytheridea, Pseudoloxoconcha, Cytheroma, Cytheretta, Nicrocytherura. Pontocyprinae, Cyprinae, Cytherurinae, Cyprinae, n.subfams.] Film, L.S.U.

- 1895. DIE OSTRACODEN. In: Reports on the Dredging Operations off the West Coast of Central America to the Calapagos, to the West Coast of Mexico, and in the Gulf of California, in charge of Alexander Agassiz, carried on by the U.S. Fish Commission Steamer "Alhatross," during the year 1891, Lieut.-Commander Z.I. Tanner, U.S.N. Commanding. Harvard, Museum Comparative Zool., Bull., vol.27, no.5, p.155-169, pls.1-3 [Gigantocypris, n.gen., with 2 n.sp., and I n.sp. of Conchoecia.] Film, Cornell. - 1898. DIE OSTRACODEN. In: WISSENSCHAFTLICHE ERGEBNISSE DER REISEN IIT MADAGASKAR UND OSTAFRIKA IN DEN JAHREN '7889-95 VON Dr. A. VOELTzKOW. Senckenhergische Naturf. Gesell., Frankfurt, a.m., Abhandlungen, Bd. 2l, p.257-296, pls.13-19. [18 n.sp., Eurycypris, Zonocypris, Oncocypris, n.gen.] Film, Columbia University.

- 1898. DIE OSTRACODEN [MADAGASCAR UND ALDABRA] Senckenbergische vaturf. Gesell., Arh., Bd. 21, p.257-296, pls.13-19. [Eurycypris, Oncocypris, Zonocypris, n.gen., and $18 \mathrm{n} \cdot \mathrm{sp} \cdot]$ Film, University of Illinnis. 
- 1900. DEUTSCHLANDS SÜSSWASSER-OSTRACODEN.

Zoologica, Heft 30, p.1-112, pls.1-2.]. Stuttgart. [a major monograph with the family Cypridae divided into subfamilies: Candoninae, Cyprinae, and Jliocyprinae; 8 new species. Film from Iowa.

- 1901a. OSTRACODA, MUSCHELKREBSE. Nordisches

Plankton von K. Brandt. Zool. Teil. Bd.IV, Lief 7, p.1-10, 19 figs. Kiel, Leipzig 1901. [Apparently reprinted 1927]. [Six species of Conchoecia, one of Philomedes]. Film, University of Michigan.

- 1901b. STENOCYPRIA, NOV. GEN. Zool. Anzeiger, Leipzig, vol. 24, p. 571-572. Film, L.S.U. - 1906a. OSTRACODA II: Wissenschaftlifht Ergebnisse der deutschen Tiefseeexpedition, vol.8, Lief 2, p.29-154, pls.5-35. [Thaumatocyprinae, n. subfam of Halocypridae, Thaumatocypris n.gen. Conchoecinae with Archiconchoecia $3 \mathrm{sp.,} 1$ new; Halocypris, 6 n.sp. and vars.; Conchoecia 81 sp. and vars. of which 55 are new, divided into 15 groups; Euconchoecia 3 sp. and vars.; I n.sp. of Cypridina, and $I$ of Ilyodromus. Essentially a monograph of the rialocypridae.] Film from University of Iowa. 
- 1906b. DIE OSTRACODEN DER SIBOGA-EXPEDITION.

Siboga-expeditie, Monographie XXX, 40pp., 9 pls.

[Uitkomsten op zoologisch, botanisch, oceanographisch, en geologisch gebied verzameld in Nederlandisch Oest-Indie 1899-1900 .... uitgegenen door Dr. Max Weber.]

Leiden, E. J. Brill, publ. [Sarsiellinae, Cylindroleberinae, subfams. of Cypridinidae; $17 \mathrm{n} . \mathrm{sp}$. belonging to 5 genera.]

- 1909. DIE OSTRACODEN DER DEUTSCHEN SÜDPOLAR-EXPEDITION 1901-1903. Deutsche Südpolar-Expedition 1901-1903, Im auftrage des Reichsamtes des innern herausgegeben von Erich von Drygalski. Berlin, X Band, Zoologie, II Band, pp. 51-181, pls.4-19, and text figs. [45 new species assigned to 30 genera; Isocypris, $n$. gen. Film from Crear Library.

- 1912. OSTRACODA in Das. Tierreich. Eine

Zusammenstellung und Kennzeichnung der rezenten Tierformen. Im Auftrage der Königl. Preuss. Akad. Wiss. zu Berlin. 31. Lieferung, pp. k-xxxiii, 1-434, 92 figs. Film, Iowa State College.

Müller, Otho F. 1772. OBSERVATIONS ON SOME BIVALVE INSECTS FOUND IN COMMON WATER, BY MR. MULLER, OF THE NEW ACADEMY OF SCIENCES IN BAVARIA AND THE OECONOMICAL SOCIETY AT BERN; COMMUNICATED BY R.H.A. BENNET, Esq; F.R.S. ROyal Soc., Philos. Trans. for 1771 [1772], vol.61, p.230-246, p1.7. [Indicates that these "insects" do not fit Monoculus as used by Linneus, Geoffroi and Baker, indicates 
Müller, otho F. 1772. (Continued)

that he intends to describe a new genus with several species, and figures at least one species from Fridricksdal, near Copenhagen, with some discussion of appendages]. Film, L.S.U.

1776. ZOOLOGIAE DANICAE PRODROMUS, SEU ANIMALIUM DANIAE ET NORVEGIAE INDIGENARUM CHARACTERS, NOMINA, ET SYNONYMA IMPRIMIS POPULARIJM. Evo. Havniae, p.I-xxxii, 1-282. [the genus Cypris, p.198-199, with these species briefly described in order: $\underline{C}$. pubera, laevis, vidua, candida, detecta, strigata, pilosa, fasciata, monacha, and ornata.] Film, Library of Congress.

- 1.785. ENTOMOSTRACA SEU INSECTA TESTACEA, QUAE IN AQUIS DANIAE ET NORVEGIAE REPERIT, DESCRIPSIT ET ICONIBUS ILIUSTRAVIT. Lipsiae et Havniae, $135 \mathrm{pp}$., 21 pls. [Ostracods, p.48-67, pls.3-7. Cypris desdribed in more detail, and these species in order, $\underline{c}$. detecta, ornata, laevis, fasciata, strigata, vidua, pubera, pilosa, monacha, crassa, candida; Cythere, n.gen. p.63, followed by these species: $\underline{c}$. viridis, lutea, flavida, gibba, gibbera.] Film made from Library of Congress copy? Munsey, Gordon C., Jr. 1953. A PALEOCENE OSTRACODE FAUNA FROM THE COAL BLUFF MARL MEMBER OF THE NAHEOLA FORMATION OF ALABAMA. Journ. Paleo., vol.27, no.1, p.1-20, pls. 1-4. [19 new sp., Toulminia, n.gen.] Film, L.S.U. 
Münster, G. von. 1830. UEBER EINIGE FOSSILE ARTEN CYPRIS (MÜLLER, LAMK•) UND CYTHERE (MULLER, LATREILLE, DESMAREST). Neues Jahrb. fur Min. Geol. Pal., p. 60-67. [Description of 14 Tertiary and 8 Carboniferous species. The Tertiary species were figured by Roemer 1838; the Carboniferous ones figured and redescribed by Jones $\varepsilon$ Kirkby 1865]. Film, L.S.U.

Murray, Grover, Jr., and Hussey, K. M. 1942. SOME TERTIARY OSTRACODA OF THE GENERA ALATACYTHERE AND BRACHYCYTHERE. Jour. Paleo., vol. 16, no. 2, p. 164-182, pls. 27-28, 2 text figs. Film, L.S.U.

Neave, S. A. 1939, 1940, 1950. NOMENCLATOR ZOOLOGICUS. A IIST OF THE NAMES OF GENERA AND SUBGENERA IN, ZOOLOGY FROM THE TENTH EDITION OF LINNAEUS 1758 TO THE END OF 1835. Zool. Soc. London, vol. 1, 1939, vol. 2-4, 1940, vol. 5 [1936 to 1945] 1950. L.S.U.

Netjkaia, A. I. 1952. NEW TYPES OF OSTRACODA FROM THE ORDOVICIAN DEPOSITS OF THE NORTHNESTERN PART OF THE RUSSIAN PLATFORM. In: Microfauna of the USSR, Part [vol,] 5. All-Union Petroleum Exploration and reological Research Institute Trans. [Leningrad: Vsesôiuznyi Neftínoy̌ Nauchno-isseldovateĺskiř Geology-Razvednochny In Institut, Trudy] Leningrad Moscow, n.s. fasc. 60, p.217-232, pls. 1-3. [II n.sp. and vars., Mica, n.gen.] From Stuart Levinson, Humble $\mathrm{O} i \mathrm{l}$ and Refining Co. 
Neviani, A. 1928. OSTRACODI FOSSILI D'ITALIA. I. VALLEBIAJA (CALABRIANO). Pontificia Acad. delle Sci., Rome. Memorie, Ser. 2, vol. 11, p. 1-120, 2 pls. [Sacculus, Flexus, Costa, Fimbria, Mutilus, Auris, Cylindrus described as "groups" of at least subgeneric rank, of "Cvthereis."] Film, University of Michigan.

Norman, Alfred Merle. 1861. CONTRIBUTIONS TO BRITISH CARCINOLOGY. I. CHARACTERS OF UNDESCRIBED PODOPHTHALMIA AND ENTOMOSTRACA. Ann. and Mag. Nat. Hist., ser. 3, vol. 8, p. 273-281, pls. 13, 14. [Cypridina teres n.sp., Philomedes longicornis Lilljeborg, p. 280, pl. 14]. Film, University of Chicago.

- 1862a. CONTRIBUTIONS TO BRITISH CARCINOLOGY. II. ON SPECIES OF OSTRACODA NEW TO GREAT BRITAIN. Ann. Mag. Nat. Hist. Ser. 3, vol. 9, p. 43-55, pls. 2, 3. [Cypris, 3 n.sp., Candona serrata, n.sp., Cythere, 3 n.sp., Cythere (Bairdia) 2 n.sp.] Film, University of Chicago. - 1862b. ON SPECIES OF OSTRACODA, FOUND IN NORTHUMBERLAND AND DURHAM, NEW TO GREAT BRITAIN. Tyneside Naturalist's Field Club, Trans., vol. 5, p. 143-155, pl. 3. [Cypris punctillata, Candona serrata, Cythere contorta n.sn.] The date is from Royal Soc. Catalogue; Lowndes 1931 gives the date as 1861. Film, University of Illinois. 
- 1865. REPORT OF THE CRUSTACEA [DREDGED OFF THE COASTS OF NORTHUMERLATD AI:D DURHA , 1862-1864 (1865)] In: Nat. Hist. Soc. Northumberland and Durham, Trans., vol. 1, p. 12-29, pls. 5-7. [Cythere declivis, $\underline{\text {. }}$ simplex, $\underline{C}$. avena, $\underline{C} \cdot \underline{\text { cellulosa, }} \underline{C}$. laevata, $\underline{C}$. multifora, I. Iatissima, C. Euttata, Cythereis Iimicola, $\underline{\text { C. dunel- }}$ mensis, n.spp.] It is given as 1867 by the Royal Society Catalogue, but that date is doubtless for the complete volume. The date is given in the Zoological Record, vol.2, for 1865, published in 1866 as 1865, hence it is clear it was published earlier than the Royal Soc. Catalogue indicates. I have therefore accepted the 1865 date, and it is clear it was published before Sars 1866, the date of which is frequently given as 1865 .

- 1867. REPORT OF THE COMMITTEE APPOINTED FOR THE PURPOSE OF EXPLORING THE COASTS OF THE HEBRIDES BY MEANS OF THE DREDGE. - PART II. ON THE CRUSTACEA, ECHINODERI:ATA, POLYZOA, ACTINOZOA, AND HYDROZOA. Brit. AssOc. Adv. Sci. Rept. of 36th Neeting, in Nottingham, p. 193206. [list of Crustacea, p. 197-198]. Film, L.S.U. - 1869. SHETLAND FINAL DREDGING REPORT. - PART II. ON THE CRUSTACEA, TUNICATA, POLYZOA, ECHINODERMATA, ACTINOZOA, HYDROZOA, AND PORIFERA. British AssOc. Adv. Sci. 38th. Meeting held in Norwich, August, 1868, published London 1869, p.247-336. Supplement, p. 341-342. 
- 1869 (Continued).

[Ostracods, p. 289-295; Cythere leioderma, ‥ quadrata,

C. navicula, Bythocythere tenuissima, n.spp.; Sarsiella, n.gen. S. capsula, n.sp.] Film, I.S.U.

Öpik, A. 1935a. OSTRACODA FROM THE LOWER ORDOVICIAN MEGALASPIS-LIMESTONE OF ESTONIA AND RUSSIA. Tartu, Estonia, Ülikool Geol. Inst. Toimetused, no. 44, $12 \mathrm{pp} ., 1 \mathrm{pl}$. [Conchoprimitia n.gen., 8 species, of which 5 are new.] Film, Ohio State University •

- 1935b. OSTRACODA FROM the OLd RED SANDSTONE AT TARTU, ESTONIA. Tartu, Estonia, Ülikool Geol. Inst. Tomietused no. 45, 8pp., I pl. [Seven species, 6 new]. Film, Ohio State University.

- 1935c. KUKRUSE LADEME OSTRAKOODIDEST. Eesti Loodus 1935, No. 3, p. 86-87. [Biflabellum tenerum n. gen., n.sp.] Film, Library Congress Copy.

- 1937. OSTRACODA FROM THE ORDOVICIAN UHAKU AND KUKRUSE FORMATIONS OF ESTONIA. Tartu, Estonia, Ülikool, Lood. Selts Aruand. vol. 43, no.1-2, p. 65-138, 8 figs. 15 pls. [Tallinnella, Polyceratella, Ctenotella, Uhakiella, Rakervella, Rigidella, Piretella, Hesperidella, Pseudostrepula, Pulvillites, n. gen., Piretellidae n.fam., and $54 \mathrm{n.sp.]} \mathrm{Film,} \mathrm{University} \mathrm{of} \mathrm{IIlinois.}$ 
Paeckelmann, Werner. 1922. DER MITTELDEVONISCHE MASSENKALK DES BERGISCHEN LANDES. Preuss. Geol. Landes., Abh., n.s., vol. 91, p. 1-112, pl.1. [Crustacea, p. 15-17, Herrmannella, n. subgen, of Leperditia, $\underline{H}$ - waldschmidti, n.sp.] Film, L.S.U.

Paris, P. 1916. SPHAEROMICOLA TOPSENTI n•g•n•sp., OSTRACODE COMMENSAL D'ISOPODES TROGLOBIES DU GENRE CAECOSPHAEROMA. Acad. Sciences, Paris, Comptes-rendus, vol. 163, p. 307309. Film, L.S.U.

Peck, Raymond E. 1951a. NONMARINE OSTRACODES - THE SUBFAMIIY CYPRIDEINAE IN THE ROCKY MOUNTAIN AREA. Journ. Paleo., vol. 25, no. 3, p. 307-320, pls. 48-50. [Pseudocypridina Roth redefined, 5 new species.? Film, L.S.U.

- 1951b. A NEW OSTRACODE GENUS FROM THE CRETACEOUS BEAR RIVER FORMATION. Journ. Paleo., vol. 25, no.5, p. 575-577, pl. 80. [Looneyella, n.gen., L. quadrispina n.sp.] Film, L.S.U.

Péneau, Joseph. 1927. ÉTUDES SUR LE DÉvONIEN DE LA LASSE-LOIRE. Soc. Sci. Nat. de l'Ouest de la France, ser. 4, vol.7, p. 94-122, p1.3. [Ostracods, p. 111-113, pl.3; Primitopsis ornatus, n.sp., Acrotonella? depressa, n.sp.] Film from University of Illinois. 
Peterson, James A. 1954. JURASSIC OSTRACODA FROM THE

"LOWER SUNDANCE" AND RIERDON FORMATIONS, WESTERN INTERIOR UNITED STATES. Journ. Paleo., vol. 28, no.2, p.153-176, pls.17-19, 3 text figs. [20 n.sp., Procytheridea, n.gen.] Film, L.S.U.

Philippi, A. 1840. ZOLOGISCHE BEMERKUNGEN. III. ASTEROPE, EIN NEUES GENUS DER OSTRACOPODEN. Archiv• für Naturges., Berlin, vol.6, pt.I, p.186-188, plate 3. Film, I.S.U.

Pokorný, Vladimír. 1950. THE OSTRACODS OF THE MIDDLE DEVONIAN RED CORAL IIMESTONES OF CELECHOVICE. Czechoslovakia, Státni Geologický Ustav, Sborník, Prague. Svazek XVII, p. 513-632, pls. 1-5. [In Czech and English, with Russian abst. Pribylites, Parapribylites, Svantovites, Unicornites, Čelechovites, Hanaites, Varicobairdia, n. gen. or subgen., and $19 \mathrm{n.sp.} \mathrm{or} \mathrm{subspecies.]} \mathrm{Film,}$ L.S.U.

- 1952. THE OSTRACODS OF THE SO-CALLED BASAL HORIZON OF THE SUBGLOBOSA BEDS AT HODONIN (PLIOCENE, INNER ALPINE BASIN CZECHOSLOVAKIA). Sborník Ústředního Ústavu Geologického, Svazek XIX, p.1-168, pls.1-6. [in Czech, Russian, with English summary]. Film, L.S.U.

1953. A CONTRIBUTION TO THE TAXONOMY OF THE PALEOZOIC OSTRACODS. Sborník Ústredního Ustavu Geologického, Svazek XX, oddíl paleontologický, p.213-232 (Nakladatelstvi Ceskoslovenske Akademie Ved). [Ostracoda, raised from an 
Order to a Class, and 4 new orders created: Beyrichiida, Podocopida, Myodocopida, Leperditiida.] Film of separate from Pokorný.

Polenova, E. N. 1952. OSTRACODA OF THE UPPER PART OF THE JIVETJKI FORMATION OF THE RUSSIAN PLATFORM (MIDDLE DEVONIAN FROM BORES IN THE CENTRAL VOLGA AREA). In Microfauna of the USSR., Part 5. All-Union Petroleum Exploration and Geological Institute Trans. [Leningrad. Vsesoiuznyi Neftiânoy̌ Nauchno-isseldovatel'skiY Geologo-Razvednochnyí Institut, Trudy] N.S., fasc. 60, p. 65156, pls.1-15. [Graviinae n. subfam. of Acronotellidae, Coelonellina, Zaborovia, Gravia, Marginia, Costatia, Samarella, Âmpuloides, Voronina, n.gen.; Gravia and Russia, n.subgen. of Gravia. and $42 \mathrm{n.sp.]}$ From Levinson of Humble Oil and Refining Co.

Posner 1851.

П О з е р. Острзкоды нижнего нароона запядого крыла ПОдмосковнод нот Ловины.

[Not seen, but from Polenova 1352, it is evident that it contains: Scrohiculidae, n.fam., I.ichwininae, n. subfam., Healdianella , Lichwinia, and Scrobicula, n.gen.]

[OSTRACODA FROM THF, LOWER CARRONITF.ROJS OF THE WESTERN WING

OF THE LODMOSKNOI RAVINE] 
Poulsen, C. 1934. THI: SILURIAI: FAUNAS CF NORTH GREENLAND.

I. THE FAUNA OF THE CAPE SCHUCHERT FORMATION. MEdd.

om Grønland, vol. 72 (Jubilaeumsekspeditionen Nord om

Grønland 1920-23), p.1-46, pls.1-3. Ceratocypris

symmetrica n.gen., n.sp., p. 38] Film, L.S.U.

- 1937. ON THE LOWER ORDOVICIAN FAUNAS OF EAST

GREENLAND. Medd. om Gronland, vol. 119, no.3, 72 pp.,

8 pls. Republished by Copenhagen Univ. Min.-Geognost. Mus., Communications paléont. No. 57, 72 pp. 8. pls.

[1937] [Ostracods, p.59-61, pl.8. Heterochilina, n.gen., H. obliqua, n.sp.] Film, L.S.U.

Přibyl, Alois. 1951. ON THE BOHEMIAN OSTRACODA OF THE FAMIIIES ENTOMOZOIDAE AND ENTOMOCHONCHIDAE. Čská Akad. ved a Uměni (Acad. Tchéque Sci.) Bull. International Prague vol. 50 (1949) 1951, [no.9], p.101-128, pls.1-2. [Entomozoacea nom. nov., for Entomidacea Schmidt 1941; Entomozoidae nom.nov., for Entomidae Jones 1873;

Entomozoinae, Bouciinae, n.subfams.; Entomozoe nom.nov. for Entomis Jones 1861, not of Herrich-Schaeffer 1856 ; Rhomboentomozoe, Pseudoentomozoe, Elpezoe, nom.nov., for Elpe Barrande 1872, not of Robineau-Desvoidy 1863 , Trubinella, nom.nov., for Hippa Barrande 1872, not of Fabricius 1787] Film, University of Illinois.

- 1953. ON SOME NEW OSTRACODES OF THE LOWER AND MIDDLE DEVONIAN OF BOHEMIA Česká Adademie véd a umerni v 
- 1953 (Continued).

Praze, Bulletin International, Prague, III Année (1951) 1953, p.15-51, pls.1-5 [Separate of this article is paged 1-37. Eleven new species; Svarogites, Boucekites, n.gen.] Film, Yale.

Pribyl, Alois, and Šnajdr, Milan. 1950. ON NEW OSTRACODA FROM THE CHOTEČ IIMESTONES - gy2 (MIDDLE DEVONIAN) OF HOLYNE NEAR PRAGUE. [Czech and English with Russian summary]. Czechoslovakia Stát. Geol. Ust., Sborník, Prague, vol. 17 (paleontology), p.101-179, pls.6-10. [Elpinella Pribyl, n.gen.; Healdiinae, n.subfam. of Bairdiidae]. Film of Levinson's copy.

Procházka, Vlad. Jos, 1893. MIOCAEN ŽIDLOCHOVICKÝ NA MORAVĔ A JERO ZVÍR̆ENA [DAS MIOCAEN VON SEELOWITZ IN MÄHREN UND DESSEN FAUNA.] Česká Akad. Praze, Rozpravy, [2] vol. 2, Heft 24, p.1-90, pls.1-3. [Ostracoda p.12, 16, 17, $20,23,25,28,30,31,45,52-57$, pls.1-2. Thirty nine species, 6 new]. Film, University of California.

Puri, Harbans S. 1952. OSTRACODE GENUS CYTHERIDEIS AND ITS ALlIES. Journ. Paleo., vol. 26, no.6, p.902-914, pls. 130-131, 14 text figs. [4 new species; Cytherideisinae, n.subfam. of Cytheridae; Cythere botellina Jones 1870, p. 157 n.name, designated genotype of Cytherideis; Sahnia and Neocytherideis, n.gen.] Film, L.S.U. 
- 1953a. THE OSTRACODE GENUS TRACHYLEBERIS AND

ITS ALLY ACTINOCYTHEREIS. Amer. Midland Naturalist, vol. 49, no. I, p.171-181, text figs, and pl.1,2. [Actinocythereis, n.gen.] Film, L.S.U.

- 1953b. TAXONOMIC COMMENT ON: "OSTRACODA FROM WELLS IN NORTH CAROLINA PART I: CENOZOIC OSTRACODA" BY F. M. SWAIN. Journ. Paleo. vol. 27, no.5, p.750-752. [Puriana Coryell and Fields, by Coryell, new name for Favella Coryell and Fielà 1937, not Jörgensen 1925, and several new specific names.] Film, I.S.U.

Randohr, F. A. 1808. ÜBER DIE GATTUNG CYPRIS MÜLL. UND DREI ZU DERSELBEN GEHÖRIGE NEUE ARTEN. Gesellschaft Naturforschender Freunde, Berlin. Magazin für die Neuesten Entdeckungen in der Desammten Naturkunde, vol. 2, p.83-93, pl.3. [ Cypris incongruens, C. gibba, C.. pellucens, n.sp.] Film, Yale.

Raymond, Percy E. 1935. LEANCHOILIA AND OTHER MID-CAMBRIAN ARTHROPODA. Harvard Univ., Mus. Compar. Zool., Bull. vol. 76, no.6, p.205-230. [Order Bradorina, nov. "leading to Ostracoda"; Families Bradoriidae Matthew, Beyrichonidae and Indianidae Ulrich and Bassler (See Ulrich and Bassler 1931). Outline of a reclassification of Cambrian Crustacea previously referred to the Ostracoda or Conchostraca]. Film, L.S.U. 
1946. THE GENERA OF FOSSIL CONCHOSTRACA - AN

ORDER OF BIVALVED CRUSTACEA. Harvard Univ •, M.us. Compar. Zool., Bul1. vol. 96, no.3, p.217-307. [On page 301, Raymond outlines the reasons why the forms referred to the Conchostraca by Ulrich and Bassler 1931 do not belong to that order, but are in fact closer to the Ostracoda, and should be placed in the order Bradorina.] Film, L.S.U.

Reuss, A. E. 1845. DIE VERSTEINERUNGEN DER BÖHMISCHEN KREIDE FORMATION. Stuttgart, Abt. I., p.1-58, pls. 1-13 [Ostracods, p.16, pl.5; 7 species of Cytherina, none new] Film, Yale.

- 1846. DIL VERSTEINERUNGEN DER BÖHMISCHEN KREID: FORMATION. Stuttgart, E. Schwoizerbart, Abt. II, p.59-148, pls. 14-51. [ostracods, p.104-105. Cytherina attenuata, faba, solenoides, mareinatac, karsteri, ornatissima, ciliata, semiplicata, spinosa, n.spp. They are figured on plate 24.] Film, Yale.

- 1854. BEITRÄGE ZUR CHARACTERISTIK DLR KREIDESCHICHTEN IN DEN OSTALPEN, BESONDERS IN GOSAUTHALE UND AM WOLFGANGSEE. K. Adad. Wissenschaften, Vienna, Nath.-Naturw. C1., Denkschr., Bd. 7, p. 1-156, pls. 1-31. [Ostracods, p. 138-142, pls.26, 27. Ten new species]. 
Richter, Reinhard. 1855. BEITRAG ZUR PÄLAONTOLOGIE DES THÜRINGER WALDES. K. Akad. Wiss, Wien, Denkschriften, Nath-Naturw. Classe, vol. 11, p.87-138, pls.1-3 [Lophryropoda (=0stracoda?), p.121-123, pl.2. Five species of Cypridina, $\underline{C}$. globulus, C. gyrata, . taeniata, and $\underline{c}$. calcarata as new.] Film, Illinois.

- 1869. DEVONISCHE ENTOMOSTRACEEN IN THURINGEN • Deutsche Geol. Gesell., Zeitschr., vol. 21, p.757-776, pls.20, 21. [9 n.sp. of Cypridina; 3 of Beyrichia.] Film, I.S.U.

Rioja, Enrique. 1942. ESTUDIOS CARCINOLOGICOS XIII. CONSIDERACIONES Y DATOS ACERA DEL GENERO ENTOCYTHERE (CRUST. OSTRACODOS) Y ALGUNAS DE SUS ESPECIES, CON DESCRIPCION DE UNA NUEVA. (1) Mexico. Instituto de Biologia, Anales, Tomo XIII, p. 685-697. [Donaldsoncythere, n.subgen. of Entosythere, and I.n.sp.] Film, I.S.U.

Robertson, David. 1880. THE FAUNA OF SCOTLAND WITH SPECIAL REFERENCE TO CLYDESDALE AND THE WESTERN DISTRICT. FRESH AND BRACKISH-WATER OSTRACODA. Pinlished by the Natural History Society of Glasgow, 35pp. [Cypris granulata, Candona euplectella n.sp., localities, but not described. An ecologic paper]. Film, Iowa State College Library 
Roemer, Friedrich Adolph. 1838. DIE CYTHERINEN DES MOLASSE-GEBIRGES. Neues Jahrbuch für Min., Geol., Pal•, p• 514-519, p1.6. [32 species, 19 new; the other previously described by Munster 1830]. Film, L.S.U.

- 1840. DIE VERSTEINERUNGEN DES NORDDEUTSCHEN KREIDEGEBIRGES. Hannover, $1840,145 \mathrm{pp} \bullet, 16 \mathrm{pls}$. [Cytherina, p.104, C. hilseana, ‥ punctatula , $\underline{\text { C. }}$ triplicata, $\underline{\text {. }}$ ovata, $\underline{\text { C. laevigata, }}$. quadrilirata, n. sp., and $\underline{C}$. subdeltoidea $\mathrm{v}$ Münster]. Film, Library of Congress.

Roth, Robert. 1928. MONOCERATINA: A NEW GENUS OF OSTRACODA FROM THE PENNSYIVANIAN OF Oklahoma. Journ. Paleo,, vol. 2, no.1, p.15-19, figs. Film, L.S.U.

- 1929a. A REVISION OF the OSTRACOD GENUS KIRKBYA AND SUBGENUS AMPHISSITES. Wagner Free Inst. Sci. Philadelphia, Publications, vol.l, p.1-55, pls.1-3. [Graphiadactyllis, n.gen.; $6 \mathrm{n} . \mathrm{sp}$. or vars of Kirkbya; 9 n.sp. or vars. of Kirkbya (Amphissites)]. Film, L.S.U. - 1929b. A CORRECTION OF GENERIC AND SPECIFIC NAMES. Journ. Paleo., vol. 3, no.3, p.292 [At the suggestion of Raymond $C$. Moore the spelling of Graphiodactyllis improperly changed to Graphiodactylus, and the spelling of the species assigned to the subgenus Amphissites changed to correspond with the gender of the subgenus instead of the gender of the genus Kirkbya.] Film, L.S.U. 
- 1929c. SOME NOTES ON THE OSTRACODE GENUS

GRAPHIODACTYLUS ROTH. Journ. Paleo., vol. 3, ru.3, p. 293-294. Film, L.S.U.

- 1929d. SOME OSTRACODES FROM THE HARAGAN MARI, DEVONIAN, OF OKLAHOMA. Jour. Paleo., vol.3, no.4, p. 327-372, pls. 35-38. [Phanassymetria, Janusella, Condracypris n.gen., and 22 new species and vars. assigned to 16 genera]. Film, L.S.U.

1933. SOME MORRISON OSTRACODA. JOURn. Paleo., vol.7, no.4, p.398-405, pl.48. [Pseudocypridina, n.gen., and 7 n.sp.] Film, L.S.U.

Roth, Robert, and Skinner, John. 1930. THE FAUNA OF THE MCCOY FORMATION, PENNSYLVANIAN OF COLORADO. Jour. Paleo., vol.4, no.4, p.332-352, pl.28-31. [Ostracods p.347-352, pl.28. Cyathus, n.gen., and 4 n.sp.] Film, L.S.U. Rouault, Narie. 1851. MÉMOIRE SUR LE TERRAIN PALÉOZOIQUE DES ENVIRONS DE RENNES. Soc. Géol. France, Bull. ser.2, no.8 (1850-1851), p.358-399. [Ostrapodes, p.377-379, figs.1-3. Leperditia, n.gen.] Film, L.S.U. Roundy, P. V. 1926. MISSISSIPPIAN FORMATIONS OF SAN SABA COUNTY, TEXAS. Part II. THE MICRO-FAUNA. U. S. Geol. Survey Prof. Paper no.146, p.5-17, pl.1. [Sansabella, Augurites, and Healdia described as new genera]. Film, L.S.U. 
Ruggieri, Giuliano. 1950a. GLI OSTRACODI DELLE SABBIE GRIGE QUATERNARIE. (MILAZZIANO) DI IMOLA. PARTIE I. Giornale di Geologia, Ser. 2, vol. 21, 1949 (1950), p.1-57, 1 pl. [Cytheretta rubra G. W. Mü]ler $1894=$ Cytherina subradiosa Roemer 1838 - genotype of Cytheretta; Cytherina edwardsi Roemer 1838, figured as Trachyleberis edwardsi, fig.4., genotype of Costa Neviani; Urocythereis, n.gen., and 6 n.sp., and vars.l Film, L.S.U.

1952. NOTA PRELIMINARE SUGLI OSTRACODI DI ALCUNE SPIAGGIE ADRIATICHE. Bologna. Università, Inst. Zool., Note del Lab. Biol. Marina di Fano, vol. 1, no.8, p.5764. [Hemicytherideis, n.gen.] Film, from Harbans Puri's personal copy.

1952b. GLI OSTRACODI DELLE SABBIE GRIGIE (MILAZZIANO) DI IMOLA, Parte II. Giorn. Geologia, Bologna, ser. 2, vol. 22, p.59-115, pls.2-9. [Paracytheropteron, n.subgen. of Cytheropteron; Rectotrachyleberis, n.subgen. of Trachyleberis; Tetracytherura, n.gen. and 7 n.sp. and vars. Film, I.S.U.

- 1953a. OSTRACODI DEL GENERE PAIJENBORCHELla

VIVENTI NEL MEDITERRANEO. SOc. Italiana di Scienze Naturali, Milan, Atti, vol. XCII, 700., 5 figs.

[Paijenborchella (Neomonoceratina) mediterranea, n.sp.]

Film, L.S.U. 
Salter, J. W. 1855. APPENDIX A. DESCRIPTIONS OF A FEW SPECIES FROM WALES AND WESTMORELAND BY J. W. Salter, in Adam Sedgwick's A synopsis of the classification of the British Paleozoic rocks with a systematic description of the British Palcozoic fossils in the Geological Museum of the University of Cambridge, by Frederick McCoy. London, J. Parker, pp. i-viii, plate I, [Cythere? umbonata, Beyrichia strangulata, n.sp.] Note date in most references is given 1852, but volume has 1855 on titlc page. Film, Library of Congress.

- 1865. NOTES ON THE SECTIONS AND FOSSILS [FROM THE. LINGULA-FLAGS OF SOUTH WALES] British Association Rept. XXXV 1865, p. 284-286, [Leperditia buprestis, p. 285] L. S. U.

Sandberger, Guido. 1845. DIE ERSTE EPOCHE DER ENTWICKELUNGSGESCHICHTF, DER ERDKÖRPERS, MIT BESONDERER BERÜCKSICHTIGUNG DER IM HERZOGTBUM NASSAU AUFGEFUNDENEN VERSTEINERTEN THIERUND PFLANZENRESTE, WELCHE DIESER ERSTEN EPOCHE DER ERD BILDUNG ANGEHÖREN. Nassauischen Verein f. Naturk. Wiesbaden, Jahrb., vol. 2, p.89-123, pls. [Ostracods, p. 120-123, pl.1. Cypridina serrato-st.riata, n.sp. designat,cd type of Richterina by Kegel 1934, p. 413]. Film, Columbia University. 
Sars, G. O. 1863. BEREINING OM EN I SOMMERN 1862 FORETAGEN ZOOLOGISK I CHRISTIANIAS OG TRONDHJEMS STIFTER: Nytt Magazin for Naturvidenskapens, Oslo, vol. 12, p.193362 [Ostracods, p.218-223; 249-252; 355-362. Cypris globosa, ‥ angustata, Cythere lacustris, Asterope norvegica, Conchoecia elegans, $\underline{C}$. borealis, Cythere drammensis, . propinqua, n.sp.]. Film, Columbia University •

- 1866. OVERSIGT AF NORGES MARINE OSTRACODER.

Norske Vidensk. -Akad. forhandlingar (1865) 1866, p.1-130. [Norwegian and Latin; this report still forms the basis for the major classification of post-Paleozoic ostracoda. Podocopa, Myodocopa, Cladocopa, Platycopa, new suborders; Conchoecidae, Polycopidae, Cytherellidae, new families; Paracypris, Pontocypris, Argilloecia, Cytheropsis, Ilyobates, Loxoconcha, Xestoleberis, Cytherura, Cytheropteron, Bythocythere, Pseudocythere, Sclerochilus, Paradoxostoma, Bradycinetus, Polycope, n.genera; and 64 new species. Film, University of Illinois.

- 1888. NYE BIDRAG TIL KUNDSKABEN OM MIDDLEHAVETS INVERTEBRATFAUNA. 4. OSTRACODA MEDITERRANEA. Archiv för Mathematik og Naturvidenskab, Oslo, vol. 12, p.173-324, pls. 1-20. [Bairdiidae n. fam., Cypridina angulata, Conchoecia pellucida, $\underline{C}$. tetragona, $\underline{C}$. striolata, Candona neglecta, Cythere amnicola n.sp.] Film, University of Ill. 
- 1889a. ON SOME FRESHWATER OSTRACODA AND COPEPODA,

RAISED FROM DRIED AUSTRALIAN MUD. Norske VidenskapsAkad, Oslo. [Christiania Vid-Selsk] Forhandlinger, No.8, p.1-79, pls.1-8. [Stenocypris n.gen., Ilyocypris

australiensis, Cypridopsis globosa n.sp.; $\underline{C}$. vidua indicated as genotype of Cypridopsis, p. 53; spelling of Erpetocypris changed to Herpetocypris.] Film, University of Illinois.

- 1889b. ON A SMALL COLLECTION OF FRESHWATER

ENTOMOSTRACA FROM SYDNEY. Norske Videnskaps-Akad.

Forhandlinger No. 9, p.3-9. [Ostracods, p.7-8]. Film, University of Minnesota.

- 1890. OVERSIGT AF NORGES CRUSTACEER, MED FOREI $\varnothing$ BIGE BEMAERKININGER OVER DE NYE ELLER MINDRE BEKJENDTE ARTER. II. (BRANCHIOPODA- OSTRACODA - CIRRIPEDIA.) Norske Videnskaps-Akademi, Oslo, Forhandlinger 1890, No.1, p.1-80 [Ostracod list p.15-21; descriptions p. 53-76. [Five new species assigned to various genera]. Film, University of Illinois.

- 1894. CONTRIBUTIONS TO THE KNOWLEDGE OF THE FRESH-WATER ENTOMOSTRACA OF NEW ZEALAND AS SHOWN BY ARTIFICIAL HATCHING FROM DRIED MUD. Norske Videnskaps-akademi, Oslo. [Videnskabs-Selskabets Skrifter I. Math-naturv KI. No. 5,] p.1-62, pls.1-8. [Ostracods p.24-48; Candonocypris, Ilyodromus, n.gen.] Film, Iowa State College. 
- 1895. ON SOME SOUTH-AFRICAN ENTOMOSTRACA RAISED

FROM DRIED MUD. Videnskabs-Selskabets Skrifter I. Math-naturv. Klasse, 1895, No. 8, p.3-55, pls.1-8. [Norske videnskaps-adademi, Oslo]. [Ostracoda, p.28-45, pls. 5,6. Cypricercus $\mathrm{n} \cdot$ gen., and $10 \mathrm{n} . \mathrm{sp} . \mathrm{J}$ Film, Iowa State College Library.

- 1898. ON MEGALOCYPRIS PRINCEPS, A GIGANTIC FRESH-WATER OSTRACOD FROM SOUTH AFRICA. Archiv för Mathematik og Naturvedenskab Oslo, vol. 20, no.8, p.1-18, pl.1. Film, University of Illinois.

- 1901. CONTRIBUTIONS TO THE KNOWLEDGE OF THE FRESH-WATER ENTOMOSTRACA OF SOUTH AMERICA, AS SHOWN BY ARTIFICIAL HATCHING FROM DRIED MATERIAL. Archiv. för Math. og Naturvidenskab. vol. 24, no.1, p.1-52, pls.1-8. Oslo. [21 n.sp. to \& genera; Amphicypris, Neocypris, n.gen.] Film, III.

- 1903. FRESH-WATER ENTOMOSTRACA FROM CHINA AND SUMATRA. Archiv för Math. og Naturv., vol. 25, no.8, p.3-44, pls.1-4. [Description of Hemicypris and Leptocypris]. Film, University of Illinois. 
- 1905. PACIFISCHE PLANKTON-CRUSTACEEN (ERGEBNISSI:

EINER REISE NACH DEM PACIFIC. SCHAUINSLAND 1896-1897). II. BRACKWASSERCRUSTACEEN VON DEN CHATHAM-INSELN. ZOOL. Jahrb. Abt. f. Syst. Geog. u. Biologie, vol. 2l, p. 371414, pls. 14-20. [Paracypris tenuis, n.sp., p. 404.] Film, University of Illinois.

- 1910. ZOOLOGICAL RESULTS OF THE THIRD TANGANYTKA EXPEDITION CONDUCTED BY Dr. W. A. CUNNINGTON, F.Z.S. 1894-1905. REPORT ON THE OSTRACODA. ZOOI. SOC. Of London, Proc. for 1910, p.732-760, pls.64-73. [Paracypria, n.gen., and 12 n.sp., 10 n.sp. of Cypridopsis, 1 of Zonnsypris, 1 of Heterocypris, 2 of Ilyocypris, 1 of Iimnocythere.] Film, L.S.U.

- 1922-1923-1925-1926-1928. AN ACCOUNT OF THE CRUSTACEA OF NORWAY. VOI. 9, CRUSTACEA. Published by the Bergen Museum, sold by Alb. Cammermeyers Forlag, Oslo. Parts I E II, Cypridinidae, Conchoeciidae, Polycopidae (Part), 1922; Parts III \& IV, Polycopidae (concluded), Cytherellidae, Cypridae (part), 1923; Partis V-XII Cypridae (concluded) and Cytheridae (part), 1925; Parts XIII \& XIV Cytheridae (continued) 1926; Parts XV E XVI Cytheridae (concluded) 1928, 277 pp., 119 pls. [A basic reclassification of the living ostracods the Podocopa in particular. Family Cypridae divided into subfamilies Fontocyorinac, Macrozyprinae, Bairdiinae, 
Darwinulidae, and Cyprinae, the latter divided into 7 groups: Candonides, Cyclocyprides, Notodromides, Ilyocyprides, Eucyprides, Cypridopsides, Paracyprides. The family Cytheridae divided into 8 subfamilies: Iimnicytherinae, Cytherideinae, Cytherinae, Cytherurinae, Loxoconchinae, Bythocytherinae, Xestoleberinae, and Paradoxostominae. The following are new genera: Leptocythere, Xenocythere, Kyphocythere, Hemicythere, Macrocythere, Cytherissa, Macrocypria.

- 1924a. THE fRESh-WATER entCMOSTRACA of the CAPE PROVINCE (UNION OF SOUTH AFRICA). PART II. OSTRACODA. South African Museum, Ann. vol. 20, p. 105-193, pls. 2-20. [Original description of Liocypris, Homocypris, Sclerocypris, Bradycypris, Paracypretta, Cyprilla, Gomphocythere.] Film, University of Illinois.

- 1924b. CONTRIBUTIONS TO A KNOWLEDGE OF THE FAUNA OF SOUTH-WEST AFRICA. I. CRUSTACEA ENTOMOSTRACA, OSTRACODA. South African Museum, Ann., vol. 20, p. 195-211, pls.21-25. [Afrocypris, n.gen., and Il n.sp. assigned to 7 genera.] Film, Iowa State College.

1926. FRESHWATER OSTRACODA FROM CANADA AND ALASKA. In Report of the Canadian Arctic Expedition 1913-1918, vol. 7, Crustacea, p.1-22, 5 pls. Ottawa. [Cypriconcha, Cytherites n.gen., and 6 n.sp.] Film, University of Virginia. 
Saussure, Henri de (1855) 1858. MÉMOIRE SUR DIVERS CRUSTACÉS NOUVEAUX DES ANTILIES ET DU MEXIQUE. (lu a la Société de Physique et d'histoire naturelle le 19 novembre 1857 ). Soc. Phys. et Hist. Nat. de Genève, Mémoires, vol. 14 (1855) 1858, p.417-495, pls.1-6. [Ostracods, p.486-490. Chlamydotheca, n.subgen., Cypris (Chlamydotheca) azteca, n.sp.] Film, Library of Congress.

Schäfer, H. W. 1945. GRUNDWASSER-OSTRACCDEN AUS GREICHENLAND. Arch. Hydrobiol. vol. 40, p.847-866, figs. [Kliella, Nannokliella, n.gen., Kliellinae, n.subfam., and new species.] Film, University of Illinois.

Schmidt, Ernst A. 1941. STUDIEN IM BÖHMISCHEN CARADOC (ZAHOǨAN-STUFE). I. OSTRAKODEN AUS DEN BOHDALECSCHICHTEN UND ÜBER DIE TAXONOMIE DER BEYRICHIACEA. Senckenb. Naturf. Ges. Abh. 454, 87pp., 2 figs., 5 pls. [Presents a major rec]assification of Palcozoic ostracods; Hollininae, Ctenentominae, Ctenonotellinae, n.subfams.of Hollinidae; Bassleratiinae, Ulrichiinae, n.subfams. of Drepanellidae; Entomidacea, n.superfam.; Ctenentoma, Parulrichia, Pseudulrichia, n.gen.; Duhmbergia n.subgen., and $16 \mathrm{n} . \mathrm{sp}$. , or new names.] Film, University of Illinois. Schweyer, A. B. 1939. THE RESULTS OF THE STUDY OF MESOZOIC AND CAINOZOIC OSTRACODA OF USSR. Neftyanoi nauchnoissledovatel'skii geologo-razvedochnyi institut, Trudy [Trans. Geol. Oil. Inst.] Series A. Fascicle 116, p.93-103. [Alexandrella, n.gen.] Film, I.S.U. 
joott, H. W. 1940. OSTRACODA FROM THE OTTER FORMATION, MISSISSIPPIAN OF CENTRAL NONTANA. Geol. Soc. Amer., Bul1. vol. 51, p.1977 (abst.) [Lochriella, n.gen., nom. nud.] Film, L.S.U.

- 1942. OSTRACODIS FROM THE UPPER MISSISSIPFIAN OF MONTANA. Journ. Paleo., vol. 16, no.2, p.152-163, pls.25, 26. [ $17 \mathrm{n} . \mathrm{sp} .$, and Lochriella, n.gen.] Film, L.S.U.

- 1944a. PERMIAN AND PENNSYLVANIAN FRESH-WATER OSTRACODES. Journ. Paleo., vol. 18, no.2, p.141-147, pls. 23-24. [Gutschickia, n.gen.] Film, L.S.U.

Scott, H. W., and Summerson, C. H. 1943. NON-MARINE OSTRACODA FROM THE LOWER FENNSYLVANIAN IN THE SOUTHERN APPALACHIANS, AND THEIR BEARING ON INTERCONTINENTAL CORRELATION. Amer. Journ. Sci., vol. 241, p.653-675, 2 pls. [Pruvostina, Hilboldtina, n.gen. and $5 \mathrm{n.sp.}$ ] Film, L.S.U.

Seguenza, Giuseppe. 1880. LE FORNAZIONI TERZTARIE NELLA PROVINCIA DI REGGIO (CALABRIA). R. Accademia nazionale dei lincei, Rome, Atti, ser, 3, Classe di Sci. Fisiche, Math, e nat., Memorie, vol. 6, p.3-443, pls. 4-17, 2 maps. [Ostracodi, p. 361-366; 40 species, and vars., 19 new]. Film, University of Illinois. 
Sharpe, Richard w. 1903. REPORT ON THE FRLSH-WATER OSTRACODA OF THE UNITED STATES NATIONAL MUSEUN, INCLUDING A REVISION OF THE SUBFAMILIES AND GENERA OF THE FANILY CYPRIDIDAE. U.S. Nat. Museum, Proc., vol. 26, p.969-1001, pls.64-69. [Spirocypris, n.gen., and 2 n.sp.]. Film, L.S.U.

Skogsberg, Tage. 1920. STUDIES ON MARINE OSTRACCDS. Part I. (CYPRIDINIDS, HALOCYPRIDS, AND POLYCOPIDS). Upsala Universitet, Zoologiska Bidrag fran Uppsala. (Zcologische Beiträge aus Uppsala) Suppl. Ed. i, 784 pF., 153 rjgs. [Cypridiniformes, Halocypriformes, Polycopiformes, Cypriformes, Cytherelliformes, now suturders, Cypridina divied into new subgenera: Cypridina, Varrula, Doloria, Macrocypridina, Siphonostra; Cypridinodes, n.subgen. of Monopia; Philomedes, Scleroconcha, n.subgen. of Philomedes. Asteropteron, n.gen. Good history of ostracod study.j L.S.U.

- 1928. STULIES ON MARINE OSTRACODS. PART II. EXTERNAL MORPHOLOGY OF THL GENUS CYTHEREIS WITH DESCRIPTIONS OF TWENTY-ONE NEW SPECIES. Calif. Acad. Sciences, Occasional papers, no.XV, p.1-155, pls.1-6. [Cytherejs, Procythereis, Pseudocythereis, n.subgen. of "Cythereis."] Film, L.S.U. 
Skogsberg, Tage. 1939. A NEW GENUS AND SPECIES OF MARINE OSTRACODS FROM SOUTH GEORGIA. Calif. Acad. Sciences, San Francisco Proceedings, vol. 33, no. 27, p.415-425, text figs. 1-13. [ Copytus caligula, n.gen., n.sp.] Film, L.S.U.

Sohn, I. G. 1950. GROWTH SERIES OF OSTRACODES FROM THE PERMIAN OF TEXAS. U. S. Geol. Survey Prof. Paper 221-C, p.33-43, pls. 7-8. [Miltonellidae n.fam. Miltonella and Aurikirkbya n.gen.] Film, L.S.U.

- 1951. CHECKLIST OF SALINITY TOLERANCE OF POST-PALEOZOIC FOSSIL OSTRACODA. Washington Acad. Sci., Journ., vol. 4l, no.2, p.64-66. [List of 80 species and varieties with occurrence]. Film, L.S.U.

- 1953. CARDINIFERELLA, N.GEN., THE TYPE OF A NEW FAMILY OF CARBONIFEROUS OSTRACODA. Wash. Acad. Sci., Jour., vol. 43, no.3, p.66-68, figs. 1-l2. Film, I.S.U.

Sohn, I. G., and Berdan, Jean M. 1952. STRATIGRAPHIC RANGE OF THE OSTRACODE GENUS PHANASSYMETRIA ROTH. Washington Acad. Sci., Journ., vol. 42, p.7-12, figs. [Pseudophanasymmetria, n.gen.] FiIm, L.S.U.

Solle, Gerhard. 1935. DIL DEVONISCHEN OSTRACODEN SPITZBERGENS. I. Leperditiidae. Norges Svalbard-og Ishavets-undersфkelser, Skrifter om Svalbard og Ishavet, Oslo, No. 64, p. 1-61, pls.1-4. [Hogmochilina, n.subgen. of Leperditia; 
Solle, Gerhard. 1935. (Continued)

Paenaequina, Holtedahlina, n.gen., and $15 \mathrm{n} . \mathrm{sp} . \mathrm{J}$ Film, Harvard.

1936. NEU BENENNUNG VON HOLTEDAHLINA SOLLE

1935. Senckenbergiana, vol. 18, p. 282. [Holtedahlites, n.name] Film, L.S.U.

Sowerby, J. de Carle. 1836. DESCRIPTIVE NOTES RESPECTING THE SHELLS FIGURED IN PLATES XI TO XXIIJ [of Dr. Fitton's paper: OBSERVATIONS ON SOME OF THE STRATA BETWEEN THE CHALK AND THE OXFORD OOLITE, IN THE SOUTH-EAST OF ENGLAND] Geol. Soc. London, Trans., (2) vol. 4, p.335-349. [Ostracods, p.344-345, pl. 2I]. Film, L.S.U.

- 1840. ORGAIIC REMAins COLLECTED by Mr. MALCOLMSON, AND DESCRIBED BY MR. JAMES DE CARLE SOWERBY. GEOI. SOC. London, Trans. vol.5, pt.3, explanation of Plate XLVII. [These are 3 unnumbered pages, starting 70 pages beyond p.754, and 23 pages before the plates. Sowerby's article and plate 47 really form a part of John G. Malcolmson's: ON THE FOSSILS OF THE EASTERN PORTION OF THE GREAT BASALTIC DISTRICT OF INDIA, Geol. Soc. London, Trans., vol.5, pt.3, p.537-575, pls. XLVI-XIVII. Sowerby's article is not listed in table of contents, nor the ostracods in the index] [Cypris cylindrica, C. subglobosa, n.spp., fossil near Munoor, India, by Malcolmson, p.550.] Film, L.S.U. 
Spivey, R. C. 1939. OSTRACODES FROM. THE MAQUOKETA SIIALE, UPPER ORDOVICIAN, OF IOWA. Journ. Paleo., vol. 13, no.2, p.163-175, pl.21. [18 n.sp. Warthinia, Zybobolboides, Marcocyproides, Ladella, n.gen.] Film, L.S.U. Spjeldnaes, Nils. 1951. ONTOGENY OF EEYRICHIA JONESI BOII. Journ. Paleo., vol. 25, no.6, p.745-755, pls.103-104, 3 figs. [Concoecia, sic. p.752. Excellent paper with. long bibliography on molts.] Film, I.S.U.

Jpizharsky, T. N. [Spijarski] 1937. OSTRACODA FROM THE KOLCHUGINO SERIES OF THE COALBEARING STRATA OF THE KUZNETSK BASIN - Russia. Central Geological and Prospecting Institute, Leningrad-Moscow, Trans. Fasc. 97, p.139171, 2 figs., 1 pl. [Tomiella, n.gen. and 8 n.sp., Leperditia 1 n.sp., Suchonellina Spijarski, 2 sp.,I new, Juchone.lIa Spijarski, S. typa, S. malachovi; Russian, english summary.] Film of personal copy.

1939. ORDER OSTRACODA. In Atlas of the Leadinf, Forms of the Fossil Fauna USSR., vol.6, Permian, p.193196, pl.XLVI. The Central Geological and Prospecting Institute, Noscow. [Suchonella, 2 sp.; Suchonellina, ] sp., Amphissites Iutkevichi n.sp.?, Sinusella n.Een.?, S. ignota n.sp.?, Tomiella, 2 sp.] Film of Stanford copy.

Stebting, Rev. Thomas R. R. 1910. GENERAL CATALOGUL OF SOUTI: AFRICAN CRUSTACEA. Sauth African Nuseum, Cape Town, Annals, vol.6, pt.4, p.281-593, pls.15-22. [Ostracoda p.495-518]. Film, Iowa state Col1 esc. 
Stephenson, Morton B. 1935. SOME MICROFOSSILS OF THE POTANIDE; NATSONI ZONE OF LOJISIANA. LOuisiana Dept. Conserv. Geol. Bull. 6, p.187-196, pl.5. [3 new species] Fi]m, L.S.U.

- 1936. SHELL STRUCTURE OF THE OSTRACODE GENUS CYTHERIDEA. Jour. Paleo., vol. 10, no.8, p.695-705, pl.94, tcxt figs. [Cytheridea redescribed, Haplocytheridea and Clithrocytheridea, n.subgen., and 1 n.sp. and 1 var.] Film, L.S.U.

- 1937. MIDDLE TERTIARY OSTRACODA OF THE GENUS CYTHERIDEA. Journ. Paleo. vol. 11, no.2, p.145-159, pl. 26, 27. [16 new species and varieties, Leptocytheridea, n.subgen.] Film, I.S.U.

- 1938. MIOCENE AND PIIOCENE OSTRACODA OF THE GENUS CYTHERIDEA FROM FLORIDA. Jour. Paleo., vol. 12, no.2, p.127-148, pls.23, 24, text figs. 1-20. [14 sp.,

12 new, Anomocytheridea, Perissocytheridea, n.gen.] Film, L.S.U.

1941. NOTES ON THE SUBGENERA OF THE OSTRACODE GENUS CYTHERIDEA, Journ. Paleo, vol. 15, no.4, p.424429, 20 text figs. [Leptocytheridea = juvenile Haplocytheridea and Phractocytheridea $=$ Haplocytheridea $; 1 \mathrm{n}$. sp.] Film, I.S.U. 
1946a. WECHES EOCENE OSTRACODA FROM SMITHVILLE, TEXAS. Jour. Paleo., vol. 20, no.4, p.297-344, pls.42-46. [Sixty-four species, 27 new, and 1 new name belonging to 23 genera, several of which are redescribed; Stephensonaria Coryel1, n.name for Pseudocythereis Jennings 1936, not Skogsberg, 1928]. Film, I.S.U.

- 1946b. GLYPTOBAIRDIA, A NEW GENUS OF OSTRACODA. Jour. Paleo., vol. 20, no.4, p.345-347, 2 text figs. [2 n.sp.] Film, L.S.U.

- 19472. NOTES ON THE OSTRACODE GENUS TRIEBELINA. Journ. Paleo., vol.21, no.6, p.577-579. [Glyptobairdia placed in synonymy of Triebelina and discussion of species.] Film, L.S.U.

- 1947b. WECHES OSTRACODA: CORRECTIONS. Journ. Paleo., vol.21, no.6, p.579-581. [Pyricythereis $=$ Buntonia; Stephensonaria = Anticythereis, and new names for several species]. Film, I.S.U.

Steusloff, A. 1894. NEUE OSTRAKODEN AUS DILUVIALGESCHIEBEN VON NEUBRANDENBURG. Deutsch. Geol. Gesell., Berlin, Zeitschr., vol. 46, p.775-787, pl.58. [30 n.sp.] Film, L.S.U. 
Stewart, Grace A. 1927. FAUNA OF THE SILICA SHALE OF LUCAS COUNTY. Ohio. Geol. Survey Bull., ser.4, no.32, 76 pp., 5 pls. [Ostracods p.13, 15, 60, pl.5. Cythere11a (?) bispinulatus n.sp.] Film, L.S.U.

- 1930. ADDITIONAL SPECIES FROM THE SILICA SHALE OF LUCAS COUNTRY, OHIO. Ohio Jour. Sci., Columbus, vol. 30, p.52-58, pl.I. [3 species, I new]. Film, L.S.U.

- 1936. OSTRACODES OF THE SILICA SHALE, MIDDLE DEVONIAN, OF OHIO. Journ. Paleo., vol. 10, no.8, p.739763, pls. 100-102. [Coelonella, Tetrasacculus, Hamiltonella, Lucasella, Menoeidina, n.gen., and 21 n.sp. and vars. assigned to various genera.] Film, L.S.U.

Stewart, Grace A., and Hendrix, W. E. 1939. OSTRACODA AS A POSSIBLE AID IN THE OLENTANGY SHALE PROBLEM. (abst•) Geol. Soc. Amer. Bull. vol. 50, p.1988-1989. [Nehdentomis dubius, Richterina symmetrica, n.spp., Franklinella, n. gen., nom.nud.] Film, L.S.U.

- 1945a. OSTRACODA OF THE PLUMBROOK SHALE, ERIE COUNTY, OHIO. Jour. Paleo., vol. 19, no.2, p.87-95, pl.10. [Punctoprimitia, Bythocyproidea, Eriella, n.gen., and 7 new species.] Film, L.S.U.

- 1945b. OSTRACODA OF THE OLENTANGY SHALE, FRANKLIN AND DELEWARE COUNTIES, OHIO. Journ. Paleo., vol. 19, no.2, p.96-115, pls.11, 12. [Bertillone11a, Bisacculus, 
Franklinella, Thrallella, Senescella, n.gen., and 27 new species]. Film, L.S.U.

Strand, Prof. Embrik (1926) 1928. MISCELLANEA NOMENCLATORICA ZOOLOGICA ET PALAEONTOLOGICA. Archiv für Naturges., Berlin, vol. 92, Abt. A, p.30-75. [Ostracods: p.40-41. Gives Carbonita Strand, nom.nov. for Carbonia Jones 1870 , nec Carbonia Robineau-Desvoidy 1863). (Gives Asteropina Strand, nom.nov. for Asterope Philippi 1840, p.186, nec Asterope Hübner 1816, p.66]. Film, University of Illinois

Straus, Hercule Eugene. 1821. MEMOIRE SUR LES CYPRIS, DE LA CIASSE DES CRUSTACÉS. Paris. Mus. Nat. d'Hist. Nat., Mém. vol.7, p.33-61, pl.1. [Good history of previous literature. First to separate ordre des ostrapodes from other groups of Entomostraca, p. 58; genus Cythere, and genus Cypris, with $3 \mathrm{n} . \mathrm{sp}$. of latter genus.] Film, University of Illinois.

Stuhlmann, Franz. 1888. VORLÄUFIGER BERICHT ÜBER EINE MIT WTTERTÜTZUNG DER KONIGLICHEN AKADEMIE DER WISSENSCHAFTEN UNTERNOMMENE REISE NACH OST-AFRICA, ZUR UNTERSUCHUNG DER SÜSSWASSERFAUNA. Akad. Wiss. Berlin, Sitzungsberichte, p.1255-1259. [strandesia, n.gen., no species, p.1260] Film, L.S.U. from Rice Institute copy. 
Sutton, A. H., and Williams, J. R. 1939. OSTRACODA FRON THE WECHES FORAATION AT BMITIVILLE, TEXAS. Journ. Paleo., vol. 13, no.6, p.56.1-574, pls.63,64. [19 species, IE of which are new; Cytheridea (Fhractocytheridea) n. subgen.] Film, L.S.U.

Swain, Frederick M. 1946a. UPPER JURASSIC OSTRACODA FROM THE COTTON VALLEY GROUP IN NORTHERN LOUISIANA: THE GENUS HUTSONIA. Journ. Paleo., vol.20, no.2, p.119-129, pls. 20-21. L Lutsonia, n.gen., with 7 n.sp.] Film, L.S.U. - 1946b. MIDDLE MESOZOTC NONMARINE OSTRACODA FROM BRAZIL AND NEW MEXICO. Journ. Palen., vol.20, no.6, p.543-555, p1s.83, 84. [9 n.sp. and vars. Paracypridea, n.subgen. of Cypridea; Pseudocypridina redefined.] Film, L.S.U.

- 1947. TERTIARY NON-MARINE OSTRACODA FROM THE. SALT LAKE FORNATION, NORTHERN UTAY. Journ. Paleo,, vol. 21 , no.6, p.518-528, pls.76-77[12 speries, 11 new; Tuberocypris, Tuberocyproides, n.ger.] Filr:, L.j.J. - 1949a. EARLY TERTIARY OSTRACODA FROM the, WESTERN INTERIOR UNITED STATES. Jour. Paleo., vol. 23, no.2, p.172-181, p1s.32-33. [4 n.sp., and vars., Cypris granulosa Sowerby designated as type of Cypridea]. Film, L.S.U. 
- 1952. OSTRACODA FROM WELLS IN NORTH CAROLINA.

Part 2. MESOZOIC OSTRACODA. U. S. Geol. Survey Prof. Paper 234-B, p.59-93, pls. 8-9. [47 species of ostracods of which 22 are new, and new genus Asciocythere] Film, L.S.U.

1953. OSTRACODA FROM THE CAMDEN CHERT, WESTERN TENNESSEE. Jour. Paleo., vol. 27, no.2, p.257-284, pls. 37-39, 21 text figs. [Camdenidea, n.gen. 29 species described, 9 n.sp., and vars.] Film, L.S.U.

Swain, F. M., and Peterson, James A. 1952. OSTRACODES FROM THE UPPER PART OF THE SUNDANCE FORMATION OF SOUTH DAKOTA, WYOMING AND SOUTHERN MONTANA. U.S. Geol. Survey Prof. Paper 243-A, 17 pp., 2 pls. [Aparchitocythere, n.gen. and 7 new species]. Film, I.S.U.

Swartz, Frank MCKim. 1932. REVISION OF THE OSTRACODE FAMILY THLIPSURIDAE, WITH DESCRIPTIONS OF NEW SPECIES FROM THE LOWER DEVONIAN OF PENNSYLVANIA. Jour. Paleo., vol.6, no.1, p.36-58, pls.10, 11. [Craterellina UEB abandoned and genotype placed in Thlipsura; Thlipsurella n.gen., 10 n.sp. and vars.] Filin, L.S.U.

- 1936. REVISION OF THE PRIMITIIDAE AND BEYRICHIIDAE WITH NEW OSTRACODA FROM THE LOWER DEVONIAN OF PENNSYIVANIA. Jour. Paleo., vol. 10, p.541-586, pls.78-89. [Primitiidae, Beyrichiidae, emended; Hollinidae, Tetradellidae, 
- 1935. (Continued).

Drunanel] [daz, Asmontellidae, Aechminidae, n.fams.,

Paraschmidtella, Milleratia, Pyxiprimitia, Parabolbina,

Tutlibairdia, Kellettina, n. gen.; 19 n.sp., and vars.;

Primitjopsidae, n.farre, proposed provisionally] Film, I..... I.

- 1945a. MID-FALEOZOIC OSTRACODA IN EXPLORATORY

WELL IN GEORGIA; MUSCLE SCARS IN LEPERDITIIDAE. GeOI. Sor. Amcr., Bull., vol. 5f, p.1205 (abst.) L Carnarvonopsis, Diagonella, n.gen?] Film, L.S.U.

- 1945b. ORIENTATION, MORPHOLOGY, AND CLASSIFICATt ON OF PALEOZOIC STRAIGHTBACKED OSTRACODA. Genl. Soc. Amer., Bull. vol. 56, p.1205-1206 [abst.]. [Aparchitacea, new super-family.] Film, L.S.U.

- 1945c. ZONAL OSTRACODA OF THE LOWER DEVONIAN IN NEW YORK AND PENNSYLVANTA. Geol. Soc. America, Bull., vol. 56, p.1204-1205 (abst.) [ Parenthetica parenthiformis, P. trisulcata and numerous other species named, but not described]. Tilm, L.S.U.

- 1949. MUSClE MARKS, HINGE AND OVERLAP FEATURES, ARD CLASSIFICATION OF SOME LEPERDITIIDAE. Jour. Paleo., vol. 23, no.3, p.306-327, 2 text figs., pls.65-67. [Leperditiinae, Isochilininac, n.subfamilies; Eoleperditia, Chevroleperditia, Teichochilina, n.gen., and 6 n.species.] Film, L.S.U. 
Swartz, F. M., and Oriel, S. S. 1948. OSTRACODA FROM MIDDLE DEVONIAN WINDOM BEDS IN WESTERN NEW YORK. Pennsylvania St. College, Tech. Paper 142, p.541-566, pls.79-81, text figs. [18 new species] Film, I.S.U.

Swartz, F. M•, and Swain, F. M. 1941. OSTRACODES OF THE MIDDLE DEVONIAN ONONDAGA BEDS OF CENTRAL PENNSYIVANIA. GeOI. Soc. Amer., Bull., vol. 52, p.381-458, pls.1-8. [40 species, of which 35 are new; Eustephanus, Stibus, Favulella, n.gen.] Film, L.S.U.

- 1942. EUSTEPHANELLA, NEW NAME FOR EUSTEPHANUS SWARTZ AND SWAIN. Jour. Paleo. vol.16, no.5, p.674. Film, L.S.U.

1946. OSTRACODA FROM THE UPPER JURASSIC COTTON VALLEY GROUP OF LOUISIANA AND ARKANSAS. Journ. PaIeo., vol. 20, no.4, p. 362-373, pls.52, 53. [9 species, 8 new; Schuleridea and Paraschuleridea, n.gen.] Film, I.S.U. Sylvester-Bradley. 1941. THE SHELL STRUCTURE OF THE OSTRACODA AND ITS APPLICATION TO THEIR PALAEONTOLOGICAL INVESTIGATION. Ann. Mag. Nat. Hist., ser.11, vol.8, p.1-33. [detailed discussion of the carapace of Cypris pubera, Candona candida, and Cythere lutea.] Film, L.S.U. 
Sylvester-Bradley, P. C. 1947. SOME OSTRACOD GENOTYPES. Ann. Mag. Nat. Hist., ser.11, vol.13, no.99, p.192-199 [with date of March 1946, but I have separate from Sylvester-3radley on which he entered date $8 \mathrm{Jan}$. 1947). [Propontocypris, Aglaiocypris, n.gen, other genera discussed: Pontocypris, Cyprois, Cypria, Bythocypris, Cytherois, Xestoleberis, Loxoconcha, Eucythere, Krithe, Cythereis. Cytherina, Erythrocypris, and Aglaia are shown to be invalid, and Cytherideis is shown to be questionable.] Film, L.S.U.

- 1948a. THE SHELI OF THE OSTRACOD GENUS MACROCYPRIS. Ann. Mag. Nat. Hist., ser. 12, vol. 1, no.1, p.65-71, 3 figs. [Superfamily Bairdiacea.] Film, L.S.U.

1948b. BATHONIAN OSTRACODS FROM THE BOUETI BED OF LANGTON HERRING, DORSET. Geol. Mag•, vol. 85, no.4, p.185-204, pls.12-15. [Subfamily Progonocytherinae, Genera Progonocythere and Lophocythere, and Superfamily Bairdiacea, plus many new species]. Film, L.S.U.

- 1948c. THE SHELl OF THE OSTRACOD GENUS BYTHOCYTHERE. Ann. Mag. Nat. Hist., ser.11, vol. 14, no.119, p.719-722, 3 figs. Film, I.S.U.

1948d. THE OSTRACODE GENUS CYTHEREIS. JOUr. Paleo., vol. 22, no.6, p.792-797, pl.122, l text fig. [Trachyleberidae, n.fam., Trachyleberinae, n.subfam., oligocythereis, n.gen., and $\underline{0}$. woodwardi, n.sp.] Film, L.S.U. 
- 1943. THE OSTRACOD GENUS CYPRIDEA AND THE

ZONES OF THE UPPER AND MIDDLE PURBECKIAN. GeOlogists Association, Proc., vol. 60, p.125-153, figs, 17-25, pls. 3,4. [Nomenclature of Cypridea, clarified; Cypris granulosa Sowerby 1836 genolectotype; and a n.sp., and var.; Cypracea, a change in spelling of Cypridacea Ulrich and Bassler]. Film, I.S.U.

- 1950. NEW NAME FOR THE OSTRACCD CROSSOPHORUS. Ann. Mag. Nat. Hist., ser.12, vol.3, p.364. [Azygocypridina, new name] Film, L.S.U.

1950b. THE SHELL OF THE OSTRACOD GENUS BAIRDIA. Ann. Mag. Nat. Hist., ser.12, vol.3, p.75l-756, 5 figs. Film, L.S.U.

- 1951a. THE RHOMBINIDAE, A NEW FAMILY OF CARBONIFEROUS OSTRACODA. Geol. Mag., vol.88, no.3, p.209-2l2, p1.11. [Palaeophilomedes, n.gen.] Film, L.S.U. - 1951b. TYPE SPECIES OF THE OSTRACOD RHOMBINA. Geol. Mag., vol. 88, p.371. Film, I.S.U.

- 1953. THE ENTOMOCONCHACEA; A NEW SUPERFAMILY OF MACROSCOPIC OSTRACODS OF UPPER PALAEOZOIC AGE. GEOI. SOC. London, Quart. Jour., vol.108, p.127-134, pls.7-8. [Entomoconchus and Cyprosina redescribed; Entomoconchacea, I. superfam.] Film, L. S.U. 
Sylvester-Bradley, P. C., and Harding, J. P. 1953. THE

NOMENCLATURE OF THE OSTRACODE GENUS CYTHERIDEIS. JOUr.

Palto., vol. 27, no.5, p.753-755. [Cytherideis unicornis

Jonus 1856 designated as type species, and Cytherideis

thus becomes synonym of Cypridea Bosquet 1852. Sahni

Furi 1952 is regarded as synonym of Neocytherideis Puri

1352; Cytherideidinae, a change in spelling of Cytheriduisinas Puri; Cytherideidae, n.fam.] Film, L.S.U.

Tasch, Paul. 1953. CAUSES AND PALEOECOLOGICAL SIGNIFICANCE OF DWAREED FOSSIL MARINE INVERTEBRATES. Journ. Paleo., vol. 27, no.3, p.356-444, pl.49, 6 text figs. [Neobeyrichiopsis, n.gen. N. emporiensis, n.sp., p.401] Film, I.. 3.U.

Teichert, Curt. 1937a. ORDOVICIAN AND SILURIAN FAUNAS FROM ARCTIC CANADA. [Reprinted from "Report of the Firth Thule Expedition 1921-24. The Danish Expedition to Arctic North America in charge of Knut Rasmussen, Ph.D." Vol. 1, No.5] Copenhagen Univ. Min. - Geognost. Mus. Communications paléont. No. 59, 169 pp., 24 pls., map. [Ostracods, p.105-122, 150-155, plates 22-24. Anisochilina, Monoetratella, Basslerites, Stuusloffina, Dihogmochilina, n.gen., and 12 n.sp., and vars., and 1 new name.l Film, L.S.U. from Princeton copy. 
Teichert, Curt. 1937b. A NEW ORDOVICIAN FAUNA FROM WASHINGTON LAND, NORTH GREENLAND. Medd. om Gronland, vol.119, no.1, 65 pp., 7 pls. Reprinted: Copenhagen Univ. Min.Geognost. Mus., Commun. paléont. no.58, 65 pp. 7 pls. [Ostracods p.43-65, pls.5, 6. 14 new species.] Film of first from L.S.U., of second from Princeton. - 1939. IIEW NAMES FOR OSTRACODE HOMONYMS. Journ. Paleo., vol. 13, no.6, p.622. [Rayella n.gen. name, for Basslerites] Film, L.S.IJ.

merquem, M. O. 1885. LES ENTOMOSTRACÉS-CSTRACODES DU SYSTEME OOLITHIQUE DE LA ZORE À AMMONITES PARKINSONI DE FONTOY (MOSELLE). Soc. Géol de France, Mém. ser. 3, vo1.4, p. 1-46, p1s.1-6. [73 species, assigned to 18 genera; Isocythere n.gen.] Film, Cornell.

Thorelund, Per. 1940. ON THE CHASMOPS SERIES OF JENTLAND AND SODERMANLAND (TVÄREN). Sweden, Sver. Geol. Unders. ser. C, no.436 (Årsbok 34, no.6), 189 pp., 56 figs., 15 pls. [Cstracods, p.151-182, pls.1-5. Balticella and Opikella, n.gen., and 17 new species of Ordovician ostracods]. Fi.m, L.S.U.

- 1948. THE CHASMOPS SERIES OF THE KULLATORP CORE. Upsala Universitet, Mineralogisk-geologisk Inst., Bull., vol. 32, p.343-373, pls.20-22. [7 n.sp., Pyxion, n.gen.] FiIm, I..S. U. 
Tolmachoff, I. P. 1926. ON THE FOSSIL FAUNAS FROM PER

SCHEI'S SERIES D FROM ELIESMERE LAND. Report of the Second Norwegian Arctic Expedition in the "Fram" 18981902, No. 38, [Ostracods, p.27-37, pls.1,2. [Published in Oslo by Norske Videnskaps-Adadeni.] [16 new species Cooperia, Ellesmeria, Paleocythere, n.gen.J Film, Made L.S.U. from U. S. Geol. Surv. copy.

- 1937. COOPERATIA, NEW NAME FOR COOPERIA TOLMACHOFF, NOT RANSOM. Jour. Paleo., vol. 11, no.1, p.78. Film, L.S.U.

Tressler, Willis I. 1937. OSTRACODA [in Mitt. XVIII von der Wallacea-Exped. Wolterek] International Revue der gesamten Hydrobiologie u Hydrographie, Bd. 34, Heft 3/5, p. 188-207, 64 figs. [Dolerocypria, n.gen., and $16 \mathrm{n} . \mathrm{sp}$. ] Film, L.S.U.

- 1939. ONYCHOCYTHERE, A NEW GENUS OF MARINE OSTRACOD FROM FLORIDA, WITH A DESCRIPTION OF ONE SPECIES.- Washington Acad. Sci., Journ., vol. 29, no.8, p.336-339. Film, L.S.U.

Tressler, W. L., and Smith, Essie M. 1948. AN ECOLOGICAI STUDY OF SEASONAL DISTRIBUTION OF OSTRACODA, SOLOMONS ISLAND, MARYLAND, REGION. Maryland Board Nat. Res., Chesapeake Biol. Lab., Publ. no. 71, 57 pp. 4 pls. [Cyprideis beaveni, Cythere triangularis, . - sclerochilus, Hemicythere truitti, $\underline{H}$. strandentia, n.spp. Sarsocythere, n.gen., S. patuxiensis, n.sp.] Film, I..S.U. 
Triebel, Erich. 1938a. D1L OSTRACODEN DER DUUTSCHLN KREIUE. 2. DIE CYTHERIDEA-ARTEN DER UNTEREN KRLIDL. Senckantersiana, vol. 20, p.471-501, pls.1-6. [Dolocytheridea, n. sibeen. of Cytheridea, and 11 n.sp.] Film, L.S.U.

- 1938b. OSTRACODEN-UNTERSUCHUNGEN. I. PROTOCYTHLRE

UND EXOPHTHALMOCYTHERE, ZWEI NLUE OSTRACODEN-GATTUNGEN AUS

DER DEIJTJCHEN KREIDE. Senckenbergiana, Bd. 20, p.178-200, pls. 1-3. Film, L.S.U.

1940. DIF: OSTRACODEN DER DEUTSCHEN KREIDE. 3. CYTHLRIDEINAE UND CYTHERINAE AUS DER UNTEREN KREIDE. Senckenbergiana, Bd. 22, f.160-227, $10 \mathrm{nls.} \mathrm{Habrocythere,}$ Apatocvthere, Isocvthereis, Platycythereis, n.pen., Cythereis rodefinnd and well illustrated, 16 n.sp.] Film, L.S.U.

- 194lb. ZUR MORPHOLOGIF UND ÖKOLOGIL DER FOSSILLN OSTRACODLN, NIT BESCHRLIBUNG EINIGER NLULR GATTUNGEN UND ARTEN. Jenckenbergiana, vol.23, p.294-4no, 15 pls.

A most important paper in relating Paleozoic to post-Paleozolc ostracoda. Ogmochoncha, Paracytheretta, n.oen.] Film, L.S.U.

- 1949a. MIKROYALÄONTOLOGISCHE KENNZEICHNUNG DER

OSTRACODENGATTUNGEN XENOCYTHLRE UND PALMENLLLA. Senckenberpiana, Bd. 30, no.4-6, 0.185-192, pls. 1,2. Film, L.S.U. 
PAIJENBCRCHELLA. Senckcnbergiana, Bd. 30, no.4/6,

p.193-203, 3 pls. [3 new species]. Film, of separate from Triebel.

- 1950a. CAMPTOCYTHERE, EINE MEUE OSTRACODEN-

GATTUNG AUS DEN: DOGGER NORDDEUTSCHLAIND. Senckenbergiana, Bd. 3I, no.3/4 p.197-208, pIs.I-3. Film, I.S.U.

- 1950b. DIE TAXONOMISCHE STELIUNG DER OSTRACODEN-

GATTUNG OGNOCONCHA UND DER LECTOTYPUS VON O. AMALTHEI. Senckenbergiana, vol. 31, no.l/2, p.113-120, pls.l-2.

- 1950c. HOMOOMORPHE OSTRACODEN-GATTUNGEN. SEn-

ckenbergiana, Bd. 31, no.5/6, p.313-330, pls.1-4.

[Cnestocythere, Schnizocythere, n.gen., and 5 n.sp.]

Film, I.S.U.

- 1951. EINIGE STRATIGRAPHISCH WERTVOLLE,

OSTRACODEN AUS DEM HOHEREN DOGGER DEUTSCHLANDS. Senckenbergische Naturf. Gesell, Frankfurt, Abh. no.845, p.87102, pls.44-49. [Pleurocythere, n.gen., and 6 n.sp. Lophocythere redescribed, and $8 \mathrm{sp.J}$ Film, I.J.U.

\section{- 1952. OSTRACODEN DER GATTUNG CYTHERETTA AUS}

DEM TERTI ÄR DES MAINZER BECKENS. Hessische Landesamt f. Bodenforschung, Notizblatt, ser.6, Heft 3, p.15-30, pls.2-5. [Cytherettinac n.subfam. of Cytheridae, 6 n.sp. and vars. of Cytheretta]. Film, I.S.U. 
Turner, Mary C. 1939. MIDDLE DEVONIAN OSTRACODA FROM OIL WELLS IN SOUTHWESTERN ONTARIO. BuIl. Amer. Paleontology, Ithaca, vol. 25, no.88, p.1-32, I pl. [Boursella, n.gen., 9 n.sp., and 2 vars.] Film, L.S.U.

Ulrich, E. O. 1879. DESCRIPTIONS OF NEW GENERA AND SPECIES OF FOSSILS FROM THE LOWER SILURIAN ABOUT CINCINNATI. Cincinnati Soc. Nat. Hist., Jour. vol.2, p.8-30, pl. [5 new species.]. Film, L.S.U.

- 1889. ON SOME POLYZOA (BRYOZOA) AND OSTRACחDA FROM THE CAMBROSILURIAN ROCKS OF MANITOBA. Canada Geol. Surv., Contrib. Micro-Pal., pt.2, p.27-57, pls.8,9. [Ostracoda, p.48-56; Eucychilina, n.gen., and n.sp. of Leperditia, Primitia, Eurychilina and Strepula. Film, L.S.U.

PALEOZOIC OSTRACODA. Cincinnati Soc. Nat. Hist., Jour. p.104-137, pls.7-10 (1890), p.173-211, pls.11-18 (1891). (Abst. by Jones, Geol. Mag. dec. 3, vol.3, no.330, p.558, 1891). [genera Ctenobolbina, Tetradella, Depranel1a, Jonesella (1890) Halliella, Pachydomella, Barychilina (1891) plus many species described as new. Ceratella nom. nud., p.I13] Film, I.S.U.

- 1891. BEECHERELLA, A NEW GENUS OF IOWER HELDERBERG OSTRACODA. Amer. Geologist, vol. 8, no.4, p.197205, p1.2. [․ carinata type, and 6 other species]. L.S.U. 
- 1892. NEW LOWER SILURIAN OSTRACODA, No. I. Amer. Geologist, vol.10, no.5, p.263-270, pl.10. [Schmidtella, n.gen. and 11 new species]. L.S.U. - 1894 (1897). THE LOWER SILURIAN OSTRACODA OF MINNESOTA. Ninnesota Geol. and Nat. Hist. Surv., Report vol. 3, pt.2, chap.7, p.629-693, pls.43-46. [advance edit. 1894] [Provisional Classification of Paleozoic Ostracods, p.632; Leperditella, Primitiella, Dicranella, Dilobella, Ceratopsis, Macronotella, Krausella described as new gen. and spelling of Depranella changed to Drepanella. Many new species.] Film, L.S.U.; Iibrary copy has date of 1897 .

- 1900. NEW AMERICAN PALEOZOIC OSTRACODA. NO. I. CTENOEOIBINA AND KIRKBYA. Cincinnati Soc. Nat. Hist., Jour. vol. 19, p.179-186, pl.8. [8 n.sp. of Ctenobolbina; of Kirkbya] Film, University of Illinois.

- 1916. OSTRACODA, in FAUNA OF THE CHAPMAN SANDSTONE OF MAINE, by Henry Shaler Williams, U. S. Geol. Surv. Prof. Paper 18, p.289-293, pl.27. [3 n.sp.; Zygobeyrichia, n.gen.] Film, L.S.U.

Ulrich, E. O., and Bassler, R. S. 1904. SYSTEMATIC PALEONTOLOGY OF THE MIOCENE DEPOSITS OF MARYLAND. OSTRACODA. Maryland Geol. Surv. Miocene Report, p.98-130, pls.35-38. Film, I.S.U. 
1906. NEW AMERICAN PALEOZOIC OSTRACODA. NOTES

AND DESCRIPTIONS OF UPPER CARBONIFEROUS GENERA AND

SPECIES. United States Nat. Mus., Proc., vol.30, p.

149-164, pl.11. [Paraparchites, n.gen. and II n.sp. and vars. assigned to several genera: Kirkbyidae, Leperditellidae, n.fams.] Film, L.S.U.

- 1908. NEW AMERICAN PALEOZOIC OSTRACODA. PRE-

LIMINARY REVISIOH OF THE BEYRICHIIDAE, WITH DESCRIPTIONS

OF NEW GENERA. U. S. National Nuseum, Proc., vol.35, p.277-340, figs. 1-61, pls.37-44. [Steusloffia, n.subgen. of Beyrichia; Scofieldia, Treposella, Hollina, Kloedenella, Kiesowia, Kirkbyina, Jonesina, n•gen •; Kloedenellinae, n. subfam. of Beyrichiidae]. Film, L.S.U.

1913a. SYSTEMATIC PALEONTOLOGY, LOWER DEVONIAN. OsTRACODA. Maryland Geol. Surv. Lower Devonian volume, tit]e, p.15, descriptions, p.513-542, pls.95-98. [24 n.sp. and vars.; Craterellina, Mesomphalus, n.gen.] Film, I.S.U.

- 1923a. PALEOZOIC OSTRACODA: THEIR MORPHOLOGY, CLASSSIFICATION, AND OCCURRENCE. Maryland Geol. Surv. Silurian vol. p.271-391, figs.11-26. Baltimore. [Saffordella, Eridoconcha, Haploprimitia, Laccoprimitia, Euprimitia, Bolbibollia, Paraechmina, Acronotella, Coelochilina, Apatochilina, Apatobolbina, Zygobolta, Zysobolhina, Zygosella, Bonnemaia, Plethobolbina, 
- 1923a (Continued).

Mastigobolbina, Kloedenia, Welleria, Dropanellina, Dibolhina, Eukloedenella, Dizygopleura, Mauryula, Acanthoscapha, Cocloch!lina, negen.; Avamehitidae, Primitiidae, Surychilininas, Zygobolbidau, Zyeobolbinae, Kloedeninav, Drupanellinae, Kloedenellidae, Bairdiidae, n.fams. or subfams.; Beyrichiacea, Cypridacea, Cytheracea, new superfamilies.] Film, L.S.U.

1923b. SYSTEMATIC PALEONTOLOGY OF SILURIAN

DEPOSITS (OSTRACODA). Maryland Geol. Surv., Silurian volume, p.500-704, text fig. 27, and pls.36-65. Baltimore. [Specific descriptions] Film, L.S.U.

- 1931. Cambrian bivalved crustacea of the order CONCHOSTRACA. United States Nat. Mus. Proc., vol. 78, Art. 4, p. 1-130, pls.1-10. [Many genera and species originally referred to the ostracoda are here referred to the Conchostraca.] Film, L.S.U.

Upson, M. E. 1933. THE OSTRACODA OF THE BIG BLUE SERIES IN NEBRASKA. Nebraska Geol. Surv., Bull. 8, second series, 54 pp., 4 pls. [19 n.sp. assigned to 10 genera; Triceratina, n.gen.] Film, L.S.U.

Vanderpool, H. C. 1928. FOSSILJ FROM THE TRINITY GROUP (IOWER CRETACEOUS). Journ. Paleo, vol.2, no.2, p.95107, pls.12-14. [Ten new species and varieties]. Film, I.S.U. 
Van Pelt, Herberta L. 1933. SOME OSTRACODES FROM THE BELI SHALE, MIDDLE DEVONIAN OF MICHIGAN. Jour. Paleo. vol. 7, no.3, p.325-342, pl.39. [15 species, 13 new; Ropolonellus, Hyphasmaphora, n.gen.] Film, L.S.U. Vávra, V. 1891a. KRITISCHES VERZEICHNISS DER OSTRACODEN BÖHMENS. Česká Spolednost Náuk, Prague, 1891, p.159-168. [Candonopsis, Eucypris n.gen, and subgen.] Film, Yale.

Vávra, V. [Wenzel] 189lb。 MONOGRAPHIE DER OSTRACODEN BÖHMENS. Archiv Naturw. Landes. Böhmen, Bd.8, no.3, p.i-iv, 1-118, 39 text figs. [Candonopsis, Eucypris, n.gen., and subgen., and $3 \mathrm{n} . \mathrm{sp}_{\text {. }}$ and vars.]. Film, Library of Congress. - 1895. DIE VON DR. F. STUHLMANN GESAMMELTEN SÜSSWASSEROSTRACODEN ZANZIBAR'S. Jahrbuch der Hamburgischen Wissenschaftichen Ansstalten XII, p. 1-23, 9 text figs. [Original description of Cypretta, Cypridella, Acocypris, Centrocypris; Strandesia as subgenus of Cypris, C. ( mercatorum, n.sp.] Film, Library of Congress. Indexed $8-26-48$.

- 1898a. SÜSSWASSER-OSTRACODEN. In Ergebnisse der Hamburger Magalhaensische Sammelreise, vol.2, p.1-26, 5 text rigs. [Notodromas patagonica, Candonopsis falklandica, Candonella paradisica, C. montevidea, Chlamydotheca symmetrica, n.sp.] Film, Crear Library. 
- 1898b. DIE SÜSSWASSER-OSTRACODEN DEUTSCH-OST--

AFRIKAS. In Deutsch-Ost-Afrika, Bd. IV, Die Thierwelt Ost-Afrikas und der Nachbargebiete, Berlin, p.1-28, 11 text figs. [Physocypria, n.subgen. of Cypria; 10 n.sp.] Film, Library of Congress.

- 1901. DIE OSTRACODEN VOM BISMARK ARCHIPEL.

Archiv für Naturgeschichte, Berlin, vol.67 (1), p.179186, pls.8, 9. [2 n.sp., Pontoparta, n.gen.] Film, L.S.U.

- 1906a. OSTRACODEN VON SUMATRA, JAVA, SIAM, DEN SANDWICH-INSELN UND JAPAN. (REISE VON DR. WALTER VOLZ•) Zool. Jahrb. Abt. f. Syst., Geog. u Biologie vol. 23, p.413-438, pls.24, 25. [Hungarocypris, n.gen., H. gawemulleri, n.sp., and new species of Cyprinotus (Hemicypris), Stenocypris, Cypridella, and Limnicythere.] Film, University of Illinois.

Veen, J. E. van. 1932. DIE CYTHERELIIDAE DER MAASTRICHTER TUFFKREIDE UND DES KUNRADER KORALLENKALKES VON SÜDLIMBURG. Geol. Nijnb. Genoots. v. Nederland en Koloniën, Verhandelingen. Geol. Ser. Deel IX, p.317-364, pls.1-25. [17 n.sp.; Terquemia, Ankumia, n.gen.] Film, L.S.U. 
- 1936b. NACHTRAG ZU DER BIS JETZT ERSCHIENEN

REVIJIOI DER OSTRACODEN DER NAASTRICHTER TUFEKREIDE UND DES KUNRADER KORALLENKALKES VON SÜD-LINBURG. Natuurhist. Maanb1., Jg.25, no.11-12, p.170-188. [ [Staringia, nom. nov. for Terquemia Veen 1932; 5 n.sp. Cythere; 2 Cytheridea;

2 Nonoceratina; 1 Krausella; 2 Phasassymetria.] Film, L.S.U.

Vejdovský, Fr. IEEC. DRUHÁ SLAVNOSTMÍ PREDNÁSKA. O PUVODU FAUNY STUDNIENÉ. Česká Společnost Náuk, Prague, Dne 3 Cervna 1880 [Böhm. Gesell. Wiss. Jber. 1880] p.XLIXLVI [Cypris eremita, n.sp.p.L] Film, from Yale. - 1882. THIERISCHE ORGAIISMEN BRUNHENWASSER VON PRAG. Prag, 1882, 4to, 70pp., 8 pls. [Ostracoda p. 64, 55, p1.7, [Typhlocypris, described as subgenus of Cypris; Cypris eremita V́ejdovský 1880, p.XIIX-IVI as subgenotype]. Film, University of Illinois.

Vernet, H. 1878. ACAITHOPUS, UN NOVEAJ GENERE D'OSTRACODES. Soc. Vaudoise des Sci. Nat., Lausanne, Bull., vol. 15, no.80, p.506-526, pls.8,9[27-28]. Film, University of California.

Vngdes, A. W. 1890. A BIBLIOGRAPHY OF PALCOZOIC CRISTACEA FROM 1689 TO 1889 INCLUDING A LIST OF NORTH AMERICAN SPECIES AND A SYSTEMATTC ARRANGEMENT OF GENERA. U. S. Geol. Survey, Bu11. 63, 177 pp. [Bibliography: p.1-78; Entomostraca, p.153-155]. Film, L.S.U. 
1889. A CATALOGUE OF NORTH AMERTCAN PALAEOZOIC CRUSTACEA CONFINED TO THE NON-TRILOBITIC GEIERA AND SPECIES. New York Acad. Sci., Annals, vol. 5, p.1-37, p1. 1,2. Film, L.S.U.

- 1893. A CLASSED AND ANNOTATED BIBLIOGRAPHY OF THE PALAECZOIC CRUSTACEA 1689-1892. Calif. Acad. Sci., Occasional Paper. No.4, 4l2pp. Film, L.S.U.

- 1895. A SUPPLEMENT TO THE BIBLIOGRAPHY OF THE PALAEOZOIC CRUSTACEA. 'California Acad. Sci., Proc., ser. 2, vol.5, p.53-76. Film, I.S.U.

1917. PALAEOZOIC CRUSTACEA: THE PUBLICATIONS AND NOTES ON THE GENERA AND SPECIES DURING THE PAST TWENTY YEARS, 1895-1917. San Diego Soc. Nat. Hist., Trans. vol.3, no.1, 141 pp., [ostracods, pl.5] Film, University of Illinois.

- 1925. PALAEOZOIC CRUSTACLA. PART I. A BIBIIOGRAPHY OF PALAEOZOIC CRUSTACEA. PART II. A LIST OF THE GLAIRRA AND SUBGENERA OF THE TRILOBITA. PART III. A SUMMARY OF THE ORDOVICIAN GENUS CYBELE LOVÉN. San Diego Soc. Nat. Hist., Trans., vol. 4, 154 pp., 2 pls. [Bibliography, p.1-88]. Film, University of Illinois. 
Wade, Arthur. 1911. THE LANDOVERY AND ASSOCIATED ROCKS OF NORTH-EASTERN MONTGOMERYSHIRE. Geol. SOc. London, Quart. Journ. vol. 67, p. 415-459, pls.33-36. [Melanella hemidiscus, n.gen., n.sp.]

Waldschmidt, E. 1885. UEBER DIE DEVONISCHEN SCHICHTEN DER GEGEND VON WILDUNGEN. Deutsch. Geol. Gesell., Berlin, Zeitschr. vol. 37, p.907-927, pls. 37-40. [Cypridina kayseri, $\underline{C}$. splendens, n.sp.] Film, L.S.U.

Warthin, Aldred S., Jr. 1930. MICROPALEONTOLOGY OF THE WETUMKA, WEWOKA, AND HOLDENVILLE FORMATIONS. Oklahoma Geol. Surv. Bull. no.53, 95 pp., 7 pls. [Ostracods, p.55-80,pls.4-7. [Cornigella n.gen., and $14 \mathrm{n} . \mathrm{sp} \cdot]$ Film, I:S.U.

- 1934. COMMON OSTRACODA OF THE TRAVERSE GROUP. Michigan Univ., Museum Pal., Contrib. vol.4, no.12, p.205-226, pl.1. [Euglyphella, n.gen.; 13 n.sp.] Film, L.S.U. - 1937. BEYRICHIACEA. In Type Invertebrate Fossils of North America (Devonian) Unit 9a, Cards 1-106. Philadelphia, Wagner Free Inst. Sci. L.S.U. - 1942. LEPERDITACEA. In Type Invertebrate Fossils of North America (Devonian) Unit 9b, Cards 1-14. Philadelphia. Wagner Free Inst. Sci. L.S.U. 
1945. THLIPSURIDAE. In Type Invertebrate Fossils of North America (Devonian) Unit 9c, Cards 1-82. Philadelphia. Wagner Free Inst. Sci. I.S.U. - 1948. OSTRACODE GENOTYPES DESIGNATED BY S. A. MILLER. Journ. Paleo., vol. 22, no.5, p.645-646. [Primitia, Bollia, Halliella, Moorea.] Film, I.S.U. Weingeist, Leo. 1949. THE OSTRACODE GENUS EUCYTHERURA AND ITS SPECIES FROM THE CRETACEOUS AND TERTIARY OF THE GULF CoAst. Journ. Paleo., vol. 23, no.4, p.364-379, pl.73. [Ravision of genus and 12 new species]. Film, L.S.U. Wetherby, A. G. 188I. DESCRIPTION OF NEW FOSSIIS FROM THE LOWER SILURIAN AND SUBCARBONIFEROUS ROCKS OF OHIO AND KENTUCKY. Cincinnati Soc. Nat. Hist., Journ. vol.4, no.1, p.77-84, pl.2. [Isochilina jonesi, n.sp., p.80] Film, L.S.U.

Whidborne, G. F. 1889, 1890. A MONOGRAPH OF THE DEVONIAN FAUNA OF THE SOUTH OF ENGIAND. Palaeontographical Soc. Mon., pt.1, p.1-46 (1889), pt.2, p.47-154, pls.5-15. [Cypridinella cacca n.sp. p.46; Polycope devonica n.sp. p.48, var. oblonga, concinna, p.49, P. hughesiae, p.50, n.sp. and vars.; Cyprosinidae n.fam., p.52.] Film, L.S.U. 
Wilson, Charles W., Jr. 1935. THE OSTRACODE FAUNA OF THE BIRDSONG SHALE, HELDERBERG, OF WESTERN TENNESSEE. JOur. Paleo., vol. 9, no.8, p.629-646, pls.77-78. [Placentella, Eucraterellina, Rothella, n.gen. and 24 n.sp.] Film, I. S.U.

Wiman, Carl. 1902. STUDIEN ÜBER DIE NORDBALTISCHE SILURGEBIET. Upsala Universitet. Mineralogisk-geologiska Institut, Bu11. vol.6, pt.1, no.11, p.12-76, pls.1-4. [Ostracods?, p.45-49. Aparchites? 2 n.sp.; Beyrichona? $3 \mathrm{r} . \mathrm{sp} \bullet$; Jellula fallax, n.gen.?, n.sp., Bradorona, Indiana?, Hipponicharion, each 1 n.sp.] Film, University of Illinois.

Zalányi, Béla. 1929. MORPHO-SYSTEMATISCHE STUDIEN ÜBER FOSSILE MUSCHELKREBSE. Geologica Hungarica. Series Palaeontologica, Fasc. 5, p.1-153, pls.1-4, 53 text figs. [I.ineocypris, n.gen. I. trapezoidea n.sp., and 16 n.sp. of various genera.] Film, University of Chicago.

- 1944 [Dr. Tordai Zalanyi Bela]. MAGYARORSZAGI

NEOGEN OSTRACODAK [Neogene ostracoden in Ungarn I. TeiI], .Geologica Hungarica, Ser. Pal., Fasc. 21, p.1-184, pls. 1-.. [30 n.sp., Amplocypris. and Thaminocypris, n.gen.] Film, University of. California. 
Zaspelova, V. S. 1952. OSTRACOEA OF THE FAMILY DREPANELI.TDAE FROM THE UPPER DEVONIAN DEPOSITS OF THE RUSSIAN PLATFORM (NORTHERN RUSSIA). In Microfauna of the USSR., Vol.5, All-Union Petroleum Exploration and Geological Research Institute [Vsesoiuznyi Neftianoi Nauchno-isseldovatelskii Geology-Razvednochnyi Institut, Trudy] Trans., Leningrad-Moscow. n.s. fasc. 60, p.157-216, pls.1-11, chart. [Neodrenanullinae, n.subfam., Neodrepanella, Tetracorne]1a, Limbatula, Bicornella, n.gen.; Nodellinae, n.subfam., Nodella, Pseudonodella, Subtella, Schweyerina, Acanthodella, n.gen., and $45 \mathrm{n} . \mathrm{sp}$. and vars. From Stuart Levinson, Humble oil and Refining Co.

Zenker, W. 1854. MONOGRAPHIE DER OSTRACODEN. Archiv für Naturgeschichte, Berlin, Bd. XX, p.1-87, pls.1-6. [Cypria n.sukgen. of Cypris, p.77, Cyprois n.gen., p.80] Film, University of Illinois. 
ACADÉMIE DES SCIENCES. Paris.

- Comptes-rendus hebdomadaires des seances. $1835 t$ Paris 1916.

ACADÉMIE ROYAIE DES SCIENCES, DES LETTRES ET DES BEAUX-ARTS DE BELGIQUE. Brussels.

- Mémoires couronnés et mémoires des Savants étrangers. Bosquet 1852; Koninck 1841.

ACADEMY OF NATURAL SCIENCES OF PHILADELPHIA.

- Proceedings. $1841 t$

Conrad 1843.

R. ACCADEMIA NAZIONALE DEI LINCEI. Rome.

- Atti.

- Classe di science fisiche, mathematiche e naturali. Memorie, Series 3, v.1-19, 1876-1884. Seguenza 1880.

ACCADEMIA PONTANIANA. Naples.

- Atti. 1820, Costa 1849.

AKADEMIE DER WISSENSCHAFTEN. Berlin.

- Abhandlungen. 1804-1907. Claus 1892.

- Sitzungsberichte. 1882-1921. Stuhlmann 1888. 
AKADEMIE DER WISSENSCHAFTEN • Munich.

(Koeniglich bayerische akademie der wissenschaften, etc.

- Abhandlungen.

F1scher 1855 .

AKADEMIE DER WISSENSCHAFTEN. Vienna.

- Mathematische-naturwissenschaftliche classe.

- Denkschriften. $1850 t$

Reuss 1854; Richter 1856.

- Sitzungsberichte. 18481

Daday 1910b.

ADADEMIIA NAUK SSSR. Leningrad.

- Comptes rendus (Doklady) de l'Académie des sciences de I'URSS. 1934T

Bronstein 1939.

- Mémoires.

Fischer 1854 .

- Zoologicheskir museř.

- Ezhegodnik... Annuaire du Musée zoologique

de 1'Académie des sciences. 1-32: 1896-1931.

Hirschmann 1916.

AMERICAN ACADEMY OF ARTS AND SCIENCES. BOston.

- Proceedings. $1845 t$

Dana 1949. 
AMERICAN GEOLOGIST. Minneapolis. 1888-1905.

Jones 1889; Ulrich 1891, 1892.

AMLRICAN JOURNAL OF SCIENCE. New Haven. 1EIF:

Harlton 1929b; Scott and Summerson 1943.

AMERICAN MIDLAND NATURALIST. Notre Dame, Indiaia, 10091

Coryell and Billings 1932; Coryell and Bonth i233;

Coryell and Rngatz 1932; Coryell and Sample !o?2;

Turtos 1936b; Ilarris and Lalicker 1932; Hof 111, ;

Puri 19533 .

ANERICAL MUSEUN: OF NATURAL HISTORY R Nicw York.

- Arrerican Museum Novitates. 19211

Coryell and Cuskley 1934; Coryell and Fields 1937;

Coryell and Malkin 1936; Coryell, Sample and

Jennings 1935; Coryell and Williamson $] 936$.

ANRALES DES SCIENCES GÉOLOGIQUES. Paris. 1eKG-188\%. Brongniart 1876 .

ANITALS AND MAGAZINE OF NATURAL. HISTORY. London. 18381

Formed by union of Magazine of zonlogy and botany, and Companion to the Botanical Magazine. Vols.1-5, 1838-40 under name Annals of natural history; or Magazine of zoology, botany and geology.

Anderson 1939; Eaird 1846; Brady 1865, 1869a; 3rady and Robertson 1869, 1270, 1872, 1874; Chapman 1901; Jones 1850a, 
ANNAIS AND MACAZINE OF NATURAL HISTORY. (Continued) $1855 \mathrm{a}, \mathrm{b}, 1856 \mathrm{~b}, 1858 \mathrm{a}, \mathrm{b}, 1879,1881 \mathrm{~d}, 1884 \mathrm{a}, 1885 \mathrm{c}, 1888$, $\mathrm{a}, \mathrm{b}, 1889 \mathrm{~b}$; Jones and Holl 1865, 1869, 1886a, b; Jones and Kirkby 1865, 1865, 1886b; M'Coy 1849, 1851; Norman 1861, 1862a; Sylvester-Bradley 1941, (1946) 1947, 1948a, b, c, $195 \mathrm{C}$; Bold 1950a.

ARBEITEN aus dem zoologischen instituten der Universität Wien und der zoolngischen station in rrist. Vienna. Claus $1890(1891), 1891 \mathrm{~b}, 1893$.

ARCHIV FÖR MATHEMATTK OG NATURVIDENSKAR. OSIO. 18761 Sars 1888, 1898, 1901, 1903.

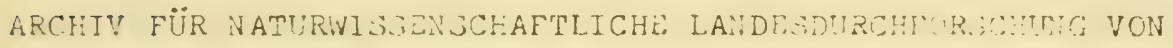
BÖHMEN. [Sec: Archiv pro prórodoveç̌̌cký vyzhum thech.] ARCHIV FÜR HYDROBIOLOGIE. Eerlin, Stuttgart. 18931 Klie 193?, 1335; Schäfer 1345.

ARCHIV FÜR NATURGESCHICHTE. Berlin. 18351

G. W. Müller 1884; Philippi 1840; Strand 192e; Zenker 1854; Vávra 1901.

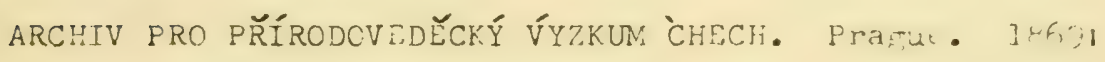
[To 1920 as: Archiv für dic naturwissenschaft] ishe Landesfurchforschung von Böhmen]. vávra 1891. 
ARCHIVES DE ZOOLOGIL LXPERIMENTALE L̇T GÉNÉRALE். Paris. KIIe 1931.

ARKIV FÖR ZOOLOGI. Stockholm. 1903'

Elof son 1939.

BATH NATURAL HISTORY AND ANTIQUARIAN FIEID CLUB. Bath.

- Proceedinfs. 1-11; 1867-1909. Jones and Sherborn 1888 .

BERWICKSHIRE' NATURALISTS' CLUB.

- History [Proceedings].

Baird 1835, 1845; Jones 1864, 1885a.

BIBLIOTHEQUE UNIVLRSLLLL. Archives des sciences physiques et naturelles. Geneva. [5 series, 1846 ]

Forel 1894 .

BOLOGNA. Universita. Instituto Zoologico.

- Note del Laboratorio di Biologia Marina di Fano. Ruggieri 1952.

BOSTON SOCIETY OF NATURAL HISTORY.

- Proceedings $1841^{t}$

Cushman 1906.

BRAZIL. Ministério da Agricultura, Departmento Nacional da Produção Mineral: Divisão de Geologia e Mineralogia.

- Boletim.

Almeida 1950. 
BRITISH ASSOCIATION FOR THE ADVANCEMENT OF SCIENCE.

- Reports. 1831-1938.

Brady 1867; Norman 1867, 1869; Salter 1865.

BRITISH MUSEUM (NATURAL HISTORY). LOndOn.

- Zoology Bulletin.

Harding and Sylvester-Bradley 1953.

BULLETINS OF AMERICAN PALEONTOLOGY. Ithaca. $1895 \mathrm{~T}$

Bradfield 1935; Jennings 1936; Morris and Hill 1952;

Turner 1939.

BUFFALO SOCIETY OF NATURAL SCIENCES. BUffalo, N.Y.

- Bulletin. 1873t

Kesling 1953d.

CALIFORNIA. University • Berkeley.

- Publications in Zoology. 1902t

Juday 1907.

CALIFORNIA ACADEMY OF SCIENCES. San Francisco.

- Proceedings.

Baker 1924; Skogsberg 1939; Vogdes 1895.

- Occasional Papers.

Vogdes 1893.

CANADA GEOLOGICAL SURVEY. (Geological and Natural History Survey)

- Contributions to Micro-paleontology.

Jones 1891; Ulrich 1889. 
CANADIAN FIELD NATURALIST.

See: Ottawa naturalist.

CANADIAN RECORD OF SCIENCE. Montreal.

Matthew 1902.

CARNEGIE. INSTITUTION OF WASHINGTON.

- Publicatinns. [Papers From the department of marine biology]. Furtos 1936.

CENTRALBLATT sEe ZENTRALBLATT.

ČESKÁ AKADEMIE VÉD A UMĚñ́ v. PRAZE (Acad. Tcheque Sci.)

- Bulletin international. 1895t

Přibyl 1951.

ČESKÁ AKADEMIE VÉD A UMĚNÍ v. PRAZE.

- Rozpravy 1892t

Procháska 1893.

ČESKÁ SPOLEČNOST NÁUK. Prague.

- V̌stník $1885+$

[German name: K. Böhrischen geselischaft der Wissenschaften.

Sitzungsherichte].

Boứek 1937; Vávra 1891; V́ejdovsky 1880.

CINCINNATI QUARTERLY JOURNAL OF SCIENCE. Cincinnati.

Vol. 1-2: 1874-1875.

Miller, 4 papers. 
CINCINNATI SOCIETY OF NATURAL HISTORY.

- Journal

Ulrich 1879, 1890-1891; Wetherby 1881.

COPENHAGEN.

Univ ersitet. Mineralogisk-geognostiske Museum.

- Communications paléontologiques. $1902 \uparrow$

Pou]sen 1937; Teichert 1937.

CZECHOSLOVAKIA. Státni Geologicky Ustav, Sbornik. Prague.

- Svazek.

Pokorný 1950; Pribyl and Šnajdr 1950.

DENISOL: UNIVERSITY. Granville, Ohio.

- Scicntific Laboratories Journal [or Bulletin].

Croneis 1939; Croneis and Bristol 1939; Croneis and

Funkhouser 1938; Croneis and Gale 1938; Croneis and Gutke

1939; Croneis and Thurman 1938; Moore 1929.

DEUTSCHE GEOLOGISCHE GESELISCHAFT. Berlin.

- Zeitschrift. $1848 t$

Krause 1889a, 1892; Lienenklaus 1894; Richter 1869;

Steusloff 1894; Waldschmidt 1885.

EESTI LOODUS. Tartu.

[Tartu Ülikooli Juures Oleva Loodusuurijate Seltsi Teataja] $1933-1938$.

Öpik 1935c. 
FIORIDA STATE BOARD OF CONSERVATION. Tallahassee.

- Geological Survey, Bulletins.

Howe, et.al., 1935; Howe 1951.

LES FONDS DE LA MER, ÉTUDE INTERNATIONALE SUR LES PARTICULARITĹS NOUVELLES DES RÉGIONS SOUS-MARINES, COMMENCÉE ET DIRIGÉE. PAR MM. L. DeFOLIN \& L. PÉRIER. 4 vols, Paris 1867-1886.

[28 short chapters with ostracods by Brady]

Film, Phila. Acad. Science.

GENOA. Museo Civico di Storia Naturale.

- Annali $1875 t$

Masi 1925-26 (1928).

GEOLOGICA HUNGARICA. Budapest.

- Series geologica 1914t; - Series paleontologica 19281 Méhes 1941; Zalányi 1929, 1944.

GEOLOGICAL SOCIETY OF AMERICA

- Bulletin 1888t

Scott 1940; Stewart and Hendrix 1939 (abst); Swartz 1945a, b, c, (abst); Swartz and Swain 1941.

- Memoirs $1934 t$

Branson 1948

- Special Papers 1934t

Bassler and Kellett 1934.

GEOLOGICAL MAGAZINE. London. $1864+$

Chapman and Sherborn 1893; Jones 1865, 1870d, 1881a, b, c, $1885 \mathrm{~d}, 1889 \mathrm{c} ;$ Jones and Kirkby 1885; Sylvester-Bradley 1948, $1951 \mathrm{a}, \mathrm{b}, \mathrm{c}$. 
GEOLOGICAL OIL INSTITUTE[USSR], TRANSACTIONS, SER. A, B.

See: LENINGRAD: VSESOIUZNYI NEFTIANOI NAUCHNO-ISSIEDOVATEĹ SKIĬ GEOLOGY-RAZVEDOCHNYI INSTITUT. TRUDY• Seriia $A, B$.

GEOLOGICAL SOCIETY OF GLASGOW.

- Transactions 1860 f

Armstrong 1871; Jones and Kirkby 1867.

GEOLOGICAL SOCIETY OF LONDON.

- Quarterly Journal. $1845 t$.

Groom 1902; Jones 1873b, 1885e, 1890a; Jones and Kirkby

1879, 1886c; Moore 1867; Sylvester-Bradley 1953; Wade 1911.

- Transactions. 1811-1856.

Fitton 1836; Sowerby 1836, 1840.

GEOLOGISTS ASSOCATION. London.

- Proceedings. $1859+$.

Anderson 1940; Jones 1885b; Jones and Kirkby 1887;

Sylvester-Bradley 1949.

GEOLOGIE. Berlin.

- Beiheft zur Zeitschrift.

Kummerow 1953.

GEOLOGISCH-MI JNBOUWKUNDIG GENOOTSCHAP VOOR NENERLAND EN KOLONI ̈̈N.

- Verhandelingen. Geologische serie. I912T. Veen 1932. 
CESELISCHAFT NATURFORSCHENDER FREUNDE. Ber? in.

- Sitzunfaherichte.

Hartwig 18996; Randohr 1808 .

FICRNALE DI GLOLCGIA. BOlOgna - Pisa.

Ruggieri 1950a, 1952b.

GREAT BRITAIN.

- Geological Survey Memoirs [Paleontology]. Jones $1856,186.1$.

GRONINGEN. Rijksunjversitejt.

- Mineralogisch-geologisch institut. Mitteilungen. Bonnema 1909.

HARVARD UNIVERSITY. Museum of Comparative Zoology.

- Bulletin.

G. W. Müller 1895; Raymond 1935, 1946.

HESSISCHE LANDESAMT FÜR BODENFORSCHUNG.

- Notizblatt, ser.6.

Triebel 1952.

ILIINOIS DEPARTMENT OF REGISTRATION AND EDUCATION.

- Illinois State Geological Survey [Division] Urbana.

- Bulletins.

Cooper 1945.

- Report of Investigations.

Cooper 1941. 
ILIINOIS. University. Urbana.

- Illinois Biological Monographs $1914 t$. Hoff 1942.

INSTITUT FRANCAIS DU PÉTROLE.

- Revue, et Annales de Combustibles Liquides. Paris. Grekoff 1951.

INTELLECTUAL OBSERVER: Review of natural history, microscopic research, and recreative science. London.

Brady 1868 .

INTERNATIONALE REVUE DER GESAMTEN HYDROBIOLOGIE UND HYDROGRAPHI . Leipzig 1908t.

Brehm 1923; Klie 1939a; Tressler 1937.

JAHRBUCH DER HAMBURGISCHEN WISSENSCHAFTLICHEN ANGTAITTN •

Hamburg. vol.1-34, 1883-1917.

Vávra 1895.

JOURNAI OF PALEONTOLOGY, Tulsa, Oklahoma. 1927 t.

Agnew 1942, 1944; Alexander 1933, 1934, 1934t, 1936; Bold 1950b; Branson 1935, 1936; Brayer 1952; Cooper 1942, 1947;

Coryell 1928a, 1928b, 1930; Coryell and Johnson 1939;

Coryell and Rozanski 1942; Coryell and Sohn 1938; Croneis and Bristol 1942; Delo 1930; Edwards 19/4; Gris 1332; 1933;

Hamilton 1944; Harding 1953; Harlton 1933; Harper and Sutton 1935; Howe 1951a; Kay 1934, 1940a, 1940b; Kellett 
JOURA AL OF PALEONTOLOGY, Tulsa, Oklahoma. 1927t. (Continued). 1933; 1935; 1936; 1943; Knight 1928; Kesling 1952b;

Levinson 1951; Nalkin 1953; Moore, Weller, Knight 1942; Morey 1935a, 1935b; Morris and Hill 1951; Munsey 1953; Peck 1951a, 1951b; Roth 1928, 1929a, 1929b, 1929c, 1929d, 1933; Roth and Skinner 1930; Scott 1942, 1944a; Spivey 1939; Spjeldnaes 1951; Stephenson 1936, 1937, 1938, 1941, 1946a, 1946b, 1947a, 1947h; Stewart 1936; Stewart and Hendrix 1345a, 1945b; Sutton and Williams 1939; Swain 1946a, 19466, 1947, 1949a, 1953; Swartz 1932, 1936; Swartz and Swain 1946; Sylvester-Bradley 1948d; Sylvester-Bradley and Harding 1953; Tasch 1953; Tolmachoff 1937; Vanderpool 1928; Var Pelt 1933; Warthin 1948; Weingeist 1949; Wilson 1935 .

KTELER MEERESFORJCHUNGEN. Kiel. Universität. Institut für meereskunde. $1936+$.

K1 ie $1936 \mathrm{~b}, 1940$.

Kosmos. (Polskie towarzystwo prsyrodników imienia Kopernika). Lemberg. vol.1-52, 1875-1927; continued in 2 series. F. Müller 1880 .

LENINGRAD. Vsesoiuznyi neftínoľ nauchno-issledovateĺ skiY geologo-razvedochny institut. Trudy. Seriia A,B.

[ = Transactions of the Geological Oil Institute, USSR.]

$[=$ All-Union Petroleum Exploration and Geological Research Institute (VNIGRI) Transactions, Leningrad-Moscow.] New Series: Netjkaia 1952; Polenova 1952; Zaspelova 1952. 
LINNEAN SOCIETY OF LONDON.

- Journal, Zoology $1855 t$.

Brady $1886 a$.

- Transactions. 1791t.

Brady $1868 \mathrm{~b}$.

LINNEAN SOCIETY OF NEW SOUTH WALES, Sydney.

- Proceedings $1875 t$

Henry 1923.

LOUISIAINA DEPARTMENT OF CONSERVATION.

- Louisiana Geological Survey, Bulletins.

Howe and Chambers 1935; Howe and Law 1936; Stephenson 1935.

LUND UNIVERSITET. Lund, Sweden.

- Acta universitatis Lundensis to 1905; Lunds universitets årsskrift after 1905.

Hadding 1913; Moberg and Segerberg 1906.

MAGAZINE OF NATURAL HISTORY. LOndon 1-9: 1828-1840.

[Merged into Annals of Natural History and later with Annals and Magazine of Natural History.]

Bean 1836.

MAGAZINE OF ZOOLOGY AND BOTANY. Edinburgh 1837-1838, v.1-2.

[Continued as Annals of Natural History: or Magazine of Zoology, Botany and Geology, vols.1-5; 1838-1840; and thence became Annals and Magazine of Natural History] Baird 1837, 1838. 
MAGYAR FÖLDRAJZI TÁRSASÁG. Budapest.

- Balaton-bisottsag. Resultate der wissenschaftichen erforschung des Balatonsces. Méhes 1911.

MARLBOROUGH COLLEGE. Marlborough, England.

- Natural History Society Reports, $1865 \mathrm{t}$. Lowndes 1931.

MARYLAND BOARD OF NATURAL RESOURCES.

- Chespeake Biological laboratory, Solomons Island. - Publications. Tressler and Smith 1948.

MARYLAND GEOLOGICAL SURVEY. Baltimore.

- Silurian volume: Ulrich and Bassler 1923a, b.

- Miocene volume: U1rich and Bassler 1904.

- Lower Devonian volume: Ulrich and Bassler 1913a.

MATHEMATIKAT ÉS TERMÉSZETTUDOMÁNYI ÉRTESITÖ. (Nagyar tudomáyos akadémia). Budapest. 1882/83t Daday 1908 .

MEDDELELSER OM GRøNLAND. Copenhagen. .

Poulsen 1934, 1937; Teichert 1937.

MEXICO. Instituto de Biologia.

- Anales.

Rioja 1942. 
MICHIGAN UNIVERSITY. Museum of paleontology.

- Contributions.

Kesling 1951, 1952, 1953a, b, c; Kesling and Hussey 1953;

Kessling and McMillan 1951; Kessling and Weiss 1953;

Warthin 1934.

THE MICROPALEONTOLOGIST. Issued $b$ : the American Museum of Natural History, New York. 19471.

Levinson 1953.

MINNESOTA. Geological and Natural History Survey.

- Annual reports.

Ulrich (1894) 1897 .

MONTHLY MICROSCOPICAL JOURNAL. LOndOn. 1869-1877.

Jones $1873 \mathrm{a}$.

I. MOSKOVSKOE OBSHCHESTVO ISPYTATELEĬ PRIRODY.

[Bulletin de la Société imperiale des Naturalistes de Moscou] $1829+$.

Eichwald 1857.

NAPLES. Stazione zoologica. Fauna und flora des golfes von Neapel. $1880+$.

21 Monographie: Ostracoden, G. W. Müller, 1894.

NASSAUISCHEN VEREIN FÜR NATURKUNDE. Wiesbaden.

- Jahrbucher. $1844 t$.

Sandberger 1845 . 
NATAL [GOVERNMENT] MUSEUM. Pietermaritzburg.

- Annals. 1909t.

Brady 1907, 1913b.

NATURAI HISTORY SOCIETY OF NEW BRUNSWICK.

- Bulletin 1882-1914.

Matthew 1889.

NATURAL HISTORY SOCIETY OF NORTHUMBERLAND, DURHAM AND NEWCASTLE' UPON TYNE.

- Transactions.

Brady 1870, 1906; Norman 1867.

NATURFORSCHENDÉ GÉSLLLSCHAFT. Bern.

- Mitteilungen. $1843^{\top}$.

Kaufmann 1900a.

NATUURHISTORISCH MAANDELAD. (Natuurhistorisch genootschap

in Limburg). Maastricht. 1912t.

Bonnema 1938; Veen 1936.

NATUURWET LNSCHAPPLIIJK TIJDSCHRIFT VOOR NEDERLANDSCH-INDI $\ddot{\text { 。 }}$

[ - to 1940 as Natuurkundige Tijdschrift vonr NederlandschIndië] 1850 .

LeRoy 1939.

NEBRASKA GLOI.OGICAL SURVEY.

- Bulletins.

Loetterle 1937; Upson 1933.

- Papers.

Johnson 1936. 
:EFTYANOI NAUCHNOISSLEDOVATEI, SKII GEOLOGO-RAZVEDOCINYI

INSTITUT. - Trudy. [Leningrad.]

Schweyer 1939.

NEUES JAHRBUCH FÜR MINERALOGIE, GEOLOGIE UND PALEONTOLOGIE. Heidelberg, Stuttgart. 1830t.

Gümbel 1874; Goldenberg 1670; Jones 1874; Münster 1830;

Roemer 1838 .

- Beilage-Band, Abteilung B.

Bouと̌ek 1936.

NEW YORK ACADEMY OF SCIENCES.

- Annals. 1877t.

Girty 1910; Vogdes 1889.

- Transactions.

Matthew 1896.

NEW YORK [STATE] NATURAL HISTORY SURVEY.

- Paleontology of New York. 1847-1892.

Hall 1852.

NEW YORK.

- Reports of the Regents of the University of the State of New York on the condition of the state Cabinet of Natural History • Albany.

Hal1 1860. 
NEW ZEALAND GEOLOGICAL SURVEY. Wellington.

- Palaeontological Bulletins. Hornibrook 1952.

NORGES SVALBARD-OG ISHAVETS-UNDERS $\varnothing K E L S E R$. OsIO.

- Skrifter om Svalbard og Ishavet. 1929t. Solle 1935.

NORSK GEOLOGISK TIDSSKRIFT. (Norsk geologisk forering) Oslo. 1905t.

Dons and Henningsmoen 1949; Henningsmoen 1950, 1953, 1953b, $1954 \mathrm{a}, \mathrm{b}$; Hessland 1954.

NORSKE VIDENSKAPS-AKADEMI. OsIo.

- Forhandlinger. Sars 1865 (1866); $1889 a, b, 1890$.

- Videnskabs-Selskabets Skrifter. Sars 1894, 1895.

NYTT MAGAZIN FOR NATURVIDENSKAPENS. Oslo. 1836t. Sars 1863.

OHIO GEOLOGICAL SURVEY. Columbus.

- Bulletins. Stewart 1927.

- Reports. Hall and Whitfield 1875. 
OHIO JOURNAL OF SCIENCE. COlumbus. 1900t.

Stewart 1930.

OHIO STATE UNIVERSITY. COIumbus.

- Ohio biological survey, Bulletins. $1913 \mathrm{~T}$.

Furtos 1933.

OKLAHOMA GEOLOGICAI. SURVEY. Norman.

- Bulletins.

Uarris 1931; Warthin 1930.

OTTAWA NATURALIST. Ottawa. 18871.

[Canadian Field Naturalist: Ottawa field naturalists club].

Foerste 1918.

PAIAEONTCGRAPHICA ITAI.ICA. Pisa. $1895 \mathrm{t}$.

Canavari 1900.

PALAEONTOGRAPHICAL SOCIETY. Iondon.

- Nonographs. 18471.

Brady, Crosskey and Robertson 1874; Jones 1849, 1857;

Jones and Kirkby 1874; Jones, Kirkby and Brady 1884;

Whidborne $1889,1890$.

FENNSYLVANIA STATE COLLEGE. Mineral iridustries experiment station. State College, Penn.

- Technical Papers. 1932t.

Swartz and Oriel 1948. 
PITTSBURGH. Carnegie Institute Museum.

- Annals. 1901/02t.

Holland 1934 .

PONTIFICIA ACCADEMIA DELLA SCIENZE. Rome.

- Momorie. 1887-1935, in 3 series.

Neviani 1928.

K. PREUSSISCHE GEOLOGISCHE LANDESANSTALT UND BERGAKADEMIE. Berlin.

- Abhandlungen. $1872 \mathrm{~T}$.

Fuchs 1915; Kummerow 1939; Natern 1929; Paeckelmann 1922.

- Jahrbuch. 1880 t.

Kegel 1932; Kummerow 1924.

REVUE CRITIQUE DE PALÉOZOOLOGIE ET DE PALÉOPHYTOLOGIE.

Paris.

Cossmann 1899.

REVUE SUISSE DE ZOOLOGIE. Geneva. 1893T.

Kaufmann 1900.

RIO DE JANEIRO. Museu Nacional.

- Archivos. 1876t.

F. Müller 1881 .

ROYAL DUBLIN SOCIETY.

- Scientific transactions. 1877-1909.

Brady and Norman 1889, 1896; Jones and Kirkby 1898. 
ROYAL GEOLOGICAL SOCIETY OF IRELAND. DubIin.

- Journal. 1833/37-1887. M. Coy 1839.

ROYAL SOCIETY OF CANADA. Ottawa.

- Proceedings and Transactions. 18821 in 3 series. Matthew 1886.

ROYAL SOCIETY OF EDINBURGH.

- Transactions $1783 \mathrm{~T}$.

Brady 1890; Latham 1933.

ROYAL SOCIETY. London.

Philosophical Transactions. 166.5t.

O. F. Müller 1772 .

ROYAL SOCIETY OF NEW ZEALAND. Wellington.

- Transactions [and Proceedings] 1868T. Hornibrook 1949.

ROYAL SOCIETY OF TASMANIA. Hobart.

[Royal Society of Van Diemen's Land through 1859]

- Proceedings and Transactions [Paper and Proseedings]. King 1855.

ROYAL SOCIETY OF VAN DIEMEN'S LAND. See: Royal Society of Tasmania. 
ROYAI, SOCIETY OF VICTORIA. MeIbourne.

- Proceedings. 1854 t in 2 series.

Chapman 1904.

RUSSIA. Central Geological and Prospecting Institute.

- Transactions.

Spizharsky 1937.

RUSSIA. Geologisheskii komitet. Leningrad.

- Vestnik.

Batalina 1924.

RUSSISCH KAISERLICHE MINERALOGISCHE GESELLSCHAFT.

Sec: I. Vserossiikoe mineralogicheskoe obschestvo.

SAN DIEGO SOCIETY OF NATURAL HISTORY. San Diego, Calif.

- Transactions. 1905t.

Vogdes 1917; 1925.

SBORNÍK ÚSTREDNÍHO ÚSTAVU GEOLOGICKÉHO. Praha.

- Svazek XIX - oddíl paleontologický.

Pokorný 1952.

SLNCKENBERGIANA. Frankfurt a.M. $1918+$.

Goerlich 1953; Krommelbein 1952; Martin 1940; Solle 1936;

Triebel 1938a, b, 1940, 1949a, b; 1950a, b, c.

SENCKENBERGISCHE NATURFORSCHENDE GESELLSCHAFT.

Frankfurt, a.M.

- Abhandlungen. 1854t.

G. W. Müller 1898; E. A. Schmidt 1941; Triebel 1951. 
SOCIETÀ ITALTANA DJ SCIENZE NATURALI. Milan.

- Atti. 1855/53t.

Ruggieri 1953a.

SOCIETÀ TOSCANA DI SCIENZE NATURALI DI PISA.

- Atti.

Canavari (1897-98) 1899.

SOCIETÁ ZOOLOGICA ITALIANA. ROme.

[1892-98 as Società Romana per fli studi zoologici].

- Bollettino. $1892 \mathrm{t}$ in 5 series.

Masi 1906.

SOCIETAS PRO FAUNA ET FLORA FENNICA. Helsingfors.

- Acta. 1875 t.

Hirschmann 1912.

- Meddelanden 1873-1924.

Hirschmann 1909.

SOCIÉTÉ DE PHYSIQUE ET D'HISTOIRE NATURELLE DE GENÈVE.

- Mémoires. 1821t.

Saussure 1858 .

SOCIÉTÉ DES SCIENCES NATUREIILES DE L'OUESTE DE IA FRANCE. Nantes.

- Bulletin. 1891t in 5 series. Péneau 1927. 
SOCIÉTÉ GÉOLOGIQUE DE FRANCE. Paris.

- Bulletin. 1830t in 5 series. Rouault 1851.

SOCIÉTÉ IMPERIALE DES NATURALISTES DE MOSCOU.

[see: I. Moskovskoe obshchestro ispytatelei prirody.]

SOCIÉTÉ NEUCHÂTELOISE DES SCIENCES NATURELLES. Neuchâte].

- Bulletin. $1843 t$.

Delachaux 1928.

SOCIÉTÉ VAUDOISE DES SCIENCES NATURELLES, Lausanne.

- Bulletin. $1842 t$.

Vernet 1878 .

SOUTH AFRICAN MUSEUM. Cape Town.

- Annals. $1898 t$

Sars 1924a, b; Stebbing 1910.

SWEDEN. Sveriges geologiska undersökning. Stockholm.

- Årsbok.

Thorslund 1940 .

SVENSKA VETENSKAPSAKADEMIENS. Stockholm.

- Handlingar 1739t in 5 series. Linnarsson 1869. 
TARTU. Estonia. - Ülikool. - Loodusuurijate Selts.

- Aruanded 1853t.

Öpik 1935a, b, 1937.

TENNESSEE STATE GEOLOGICAL SURVEY. Nashville.

- Bulletin.

Bassler 1932.

TERMÉSZETRAJZI FÜZETEK. Budapest. 1877-1902.

Daday 1895; 1898; Kertész 1893.

TEXAS. University, Austin.

- Bulletins.

Alexander 1929; Harlton 1929.

TYNESIDE NATURALIST'S FIELD CLUB. Newcastle.

- Transactions. 1846-1864.

Kirkby [and Jones] 1860; Norman 1862b.

UNITED STATES GEOLOGICAL SURVEY. Washington, D. C.

- Bulletins.

Vogdes 1890.

- Professional Papers.

Roundy 1926; Sohn 1950; Swain 1952; Swain and Peterson 1952.

UNITED STATES NATIONAL MUSEUM. Washington, D. C.

- Proceedings.

Sharpe 1903; Ulrich and Bassler 1906, 1908, 1931. 
UPSALA. Universitet. Mineralogisk-geologiska institut.

- Bulletin. 1892 .

Hessland 1949; Henningsmoen 1948; Thorsuland $1948^{\circ}$

UPSALA. Universitet.

- Zoologiska bidrag från Uppsala. 1911/12t.

Alm 1915; Elofson 1941; Ekman 1914; Skogsberg 1920.

VIDENSKABS-SELSKABETS SKRIFTER.

See: Norske Videnskaps-Akademi, Oslo.

I. VSEROSSIISKOE MINERALOGISCHESHOE OBSHCHESTVO. Leningrad.

[Materialien zur geologie Russlands. Russisch

Kaiserliche Mineralogische gesellschaft, St. Petersburg].

- Verhandlungen.

Gürich 1896.

WASHINGTON ACADEMY OF SCIENCE. Washington, D. C.

- Journal.

Bassler 1941; Sohn 1951, 1953; Sohn and Berdan 1952;

Tressler 1939.

WASHINGTON. University, Seattle.

- Publications in Biology. 1932t.

Dobbin 1941.

WISCONSIN ACADEMY OF SCIENCES, ARTS, AND LETTERS. MadisOn.

- Transactions. 1870-1932.

Narshall 1903. 
WISTAR INSTITUTE OF ANATOMY AND BIOLOGY. Philadelphia.

- Biological Survey of the Mount Desert Region. Blake 1933.

ZEITSCHRIFT FÜR GESCHIEBEFORSCHUNG. Berlin. 1925 .

Kumme row 1943.

ZEITSCHRIFT FÜR WISSENSCHAFTLICHE ZOOLOGIE. Leipzig. $1848 \mathrm{t}$. Claus 1873; Klie 1929.

ZENTRALBLATT FÜR MINERALOGIE, GEOLOGIE UND PALEONTOLOGIE, Stuttgart. 1900t [supplement to Neues Jahrbook 1925t].

ZOOLOGICA. Cassel, Stuttgart. 1887t.

Daday 1905; 1910a; G. W. Müller 1900.

ZOOLOGICAL SOCIETY OF LONDON.

- Nomenclator zoologicus.

Neave 1939, 1940, 1950.

- Proceedings 1833t.

Baird 1859; Brady 1886b, 1910, 1913; Sars 1910.

- Transactions. $1835 \mathrm{~T}$.

Brady 1866, 1878, 1897, 1898, 1902.

ZOOLOGISCH-BOTANISCHE GESELLSCHAFT. Vienna.

- Verhandlungen. 1851 t.

Claus 1874b; Chyzer 1858. 
ZOOLOGISCHE JAHRBÜCHER. Jena.

- Abtheilung für systematik, ökologie urd geographie der tiere. $1888 \mathrm{~T}$.

Klie 1936, 1939b; Müller 1890a,b, 1900; Sars 1905; Vávra 1906.

ZOOIOGISCHER ANZEIGER. Leipzig• 1878T •

hartwig I899a; Kaufmann 1892, 1900c; Klie 1938b, 1939, 1940; Müller 1901b.

ZOOLOGIST, a monthly journal of natural history. Iondon. 1843-1.916, in 4 series.

Baird 1843. 
In the arrangement of this Handbook every attempt has ben made to have it complete to the end of 1952. Such publications as have come to my attention with 1953 and 1954 dates have been included, but doubtless some have been overlooked. Since the manuscript was typed for the press, 7 additional papers have been received in which 26 new terms have been proposed, with types selected from 2 additional papers not included in the Handbook. These additions are given below:

Puri, Harbans S. Aug. 17, 1954 [1953 on title page].

CONTRIBUTION TO THE STUDY OF THE MIOCENE OF THE' FLORIDA PAN HANDLE. Part III. OSTRACODA. Florida Gool. Survey, BuII. 36, p. $215-345,17$ pls. $[45 \mathrm{n} \cdot \mathrm{sp} \cdot, 4 \mathrm{n} \cdot$ gen., 2 n.subfams.]

BRACHYCYTHERINALi PUri 1954, p.248, a subfamily of Cytheridae to include: Brachycythere and

\section{Alatacythere.}

ECHINOCYTHEREIS [Cythereis garretti Howe and McGuirt, in Howe and graduate students 1935, p.20,pl. 3 : f.17-19; pl.4,f.5,15] Puri 1954,p.259.

[Cytheridae-Trachyleberinae by Puri] Stated range Eocene to Recent.

EUCYTHLRINAE Puri 1954,p.298, a subfamily of Cytheridae to include the genus Eucythere. 
HERMANIA [H. reticulata] Puri 1954 p.267 [Cytheridae-Hemicytherinae by Puril Miocene of Florida.

MURRAYINA [M. howei] Puri 1954 p.255 [Cytheridae-Trachyleberinae by Puri] Miocene, Maryland, Florida.

ORIONINA [Cythere vaughani Ulrich and Bassler 1904, p.109,pl.38,f.25-27] Puri 1954 p.253

[Cytheridae-Trachyleberinae by Puri] Miocene, Maryland, Florida. State range Eocene to Recent.

Öpik, A. A. 1953

LOWER SILURIAN FOSSILS FROM THL "ILLALNUS BAND" HLATHCOTE, VICTORIA. Victoria Geol. Survey, Memoirs no. 19, p.9-42, pls.11-13. [15 n.sp., 3 n.gen.]

GILLATIA [G. truncata] Öpik 1953,p.34 [Beyrichiidae by Opik] Silurian, Victoria.

KAYATIA [K. prima] Öpik 1953,p.32 ["beyrichoid form" near Conchoprimitia] Silurian, Victoria.

QUADRICOLLINA [Q. initialis] Öpik 1953,p.30 [Drepanellidae, near Ulrichia by Önik] Silurian, Victoria.

Henningsmoen, Gunnar 1954. 
SILURIAN OSTRACODA FROM THF OSLO REGION, NORWAY. NOFSK Geologisk Tidsskrift, Bd. 34,h.1,p.15-71,pls.1-8 [15 n.sp., 5 n.subgen., 2 n.gen., 1 n. subfam.]

CORNIKLOEDENIA 「Drepanellina ventralis Ulrich and Bassler 1923, p.650, p1.56,f.5,6] Henningsmoen 1954 , p.31 [Beyrichiidae-Kloedeniinae] Silurian, Norway.

EOBEYRICHIA [Beyrichia (Eobeyrichia) zygophora]

Henningsmoen $1954, \mathrm{p} .22$ [as subgenus of Beyrichia by Henningsmoen] Silurian, Norway.

MITROBEYRICHIA [Beyrichia jonesii Boll 1856, p.322, figs.

1,2] Henningsmoen $2954, \mathrm{p} .27$ [as subgenus of Beyrichia] Silurian, Germany.

NEOBEYRICHIA [Beyrichia buchiana Jones $1855 \mathrm{a}, \mathrm{p} .86, \mathrm{pl}$ 5,f.1-3] Henningsmoen 1954,p.25 [as subgenus of Beyrichial Silurian, north Europe.

NODIBEYRICHIA [Beyrichia bronni Reuter 1885,p.638,p1.25, f.6A,B] Henningsmoen 1954,P.26 [subgenus of Beyrichia] Silurian drift, Germany.

SIGNETOPSIS [S. quadrilobata] Henningsmoen 1954,p.61 [Primitiopsidae by Henningsmoen] Silurian, Norway. 
TREPOSELIINAE Henningsmoen $1954, \mathrm{p} .33$, new subfamily of Beyrichiidae for the genera Treposella, Bolbiprimitia, Hibbardia, Phlyctiscapha, Nesomphalus.

VEIIBFYRTCHIA [Beyrichia moodeyi Ulrich and Bassler 1908, p.285,pl.37,f.8] Henningsmoen $1954, p .24$ [as subgenus of Beyrichia] Silurian, West Virginia, Maryland.

Sohn, I. G. 1954

OSTRACODA FROM THE FERMIAN OF THE GLASS MOUNTAINS, TEXAS. U.S. Geological Survey, Prof. Paper 264-A, 24pp., 5 pls. $[5 \mathrm{n} \cdot$ gen., $13 \mathrm{n} . \mathrm{sp} ., 1 \mathrm{n} \cdot \mathrm{subfam}$.

CERATOBAIRDIA [C. dorsospinosg] Sorn 1954,p.5 [Bairdiidae by Sohn] Permian, Texas.

CCRONAKIRKBYA [C. fimbriata] Sohn 1954,p.10 [Kirkbyidae-Kirkbyinae by Sohn] Permian, Texas.

KELLETTININAE Sohn 1954,p.14, a subfamily of Kirkbyidae for Kellettina, Kindlella and Semiplastus.

KINDLELLA [K. fissiloba] Sohn 1954, p.16 [Kirkbyidae-Kellettininae by Sohn] Permian, Texas.

imnilolus i3. sigmoides] Sohn 1954,p.17 [Miltonellidae by Sohn] Permian, Texas. 
SEMIPLASTUS [s. signatus] Sohn 1954,p.16 [Kirkbyidae--

Kellettininae by Sohn] Permian, Texas.

Triebel, Erich 1354

MALM-OSTRACODEN MIT AMPHIDONTEM SCHLOSS. Senckenbergiana vol. $35, \mathrm{no} \cdot 1-2, \mathrm{p} \cdot 3-16, \mathrm{pls} .1-4$. [Macrodentina redescribed; Amphicythere,n.gen.]

AMPHICYTHERE [A. semisulcata] TriebeI 1954,p.13

[Cytheridae-Progonocytherinae by Triebel] Jurassic, Germany.

LOXOCONCHEILA n. $\cdot$ (Crust., Ostr.). Senckcnbergiana, vol.35,no.1-2,p.17-21, 2 pls.

LOXOCONCHELLA [Loxoconcha honoluliensis Brady 1880, p.117,pl.28,f.6a-d (not figs.6e-f)] Triebel 1954, p.17-21 [Cytheridae-Loxoconchinae by Triebel] Recont, Australia.

Boll, Ernst 1856

HERR BOLI AN HERRN BEYRICH. Deutsch Geol. Gesell., Zeitschr., vol.8,p.321-324,figs.1-4 [Beyrichia ionesii, B. spinulosa, B. hians, n.spp.J Film: L.S.U.

Reuter, Georg 1885

DIE BEYRICHIEN DER OBERSTLURISCHEN DILUVIALGESCHIEBE OSTPREUSSENS. Deutsch Geol. Gesell., Zeitschr., vol.37,p.621-679,pls.25,26, and pl.opp.p.660. [17 n.sp. and vars of Beyrichia, of which B. bronni has been made type of Neobeyrichia by Henningsmoen 1954] Film: L.S.U. 
Puri, Harbans S. 1953

THE OSTRACODL GLNUS HLMICYTHERE AND ITS ALLIES. Washington Acad. Sc1. Journ. vol. 43, No. 6, p. 169-179, pls. 1,2. [2 n.sp., I new subfam.].

HEMICYTHERINAE; Puri 1953,p.172, a naw subfamily of Cytheridae to include the fenera Hemicythere, Procythereis, Caudites, Heterocythereis, and Urocythere. 




\section{LOUISIANA STATE UNIVERSITY STUDIES}

\section{Social Science Series}

1. Postell, William Dosite, The Health of Slaves on Southern Plantations, $195 \overline{1 ;} \$ \overline{3.00-0 u t}$ of Print

2. West, Robert C., Colonial Placer Mining in Colombia, 1952; \$3.00

3. Richardson, W. C., Stephen Vaughan: Financial Agent of Henry VIII, 1953:\$1.50

\section{Humanities Series}

1. Hammer, Carl, Jr., (ed.), Goethe After Two Centuries, $1952 ; \$ 2.50$ 2. Hammer, Carl. Ir., Longfellow's "Golden Legend" and
Goethe is "Faust," $1952: \$ 0.50$

3. Krumpelmarn. John T., Mark Twain and tine German Language, 1953; \$0.50

4. Uhler, John E., Morley's Canzonets f'or Two Voices, 1954: \$2.50

5. Contributions to the Humanities, 1954; $\$ 2.00$

\section{Biological Science Series}

1. Dalquest, Walter Mammals of the Mexican State of San Luis Potosi, $1953 \overline{\$} \$ 3.50$

2. Cochran. Fred D. C Cytogenetic Studies of the Species Hybrid Allium fistulosum $x$ Allium ascaloniclim and Its Backcross Progenies. $1953 \%$ \$2.00 






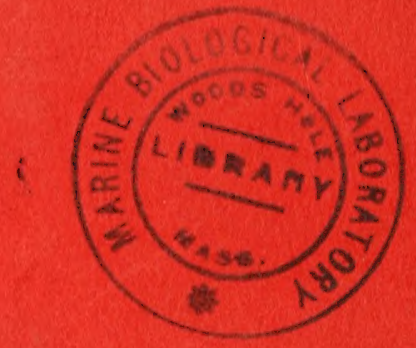

